
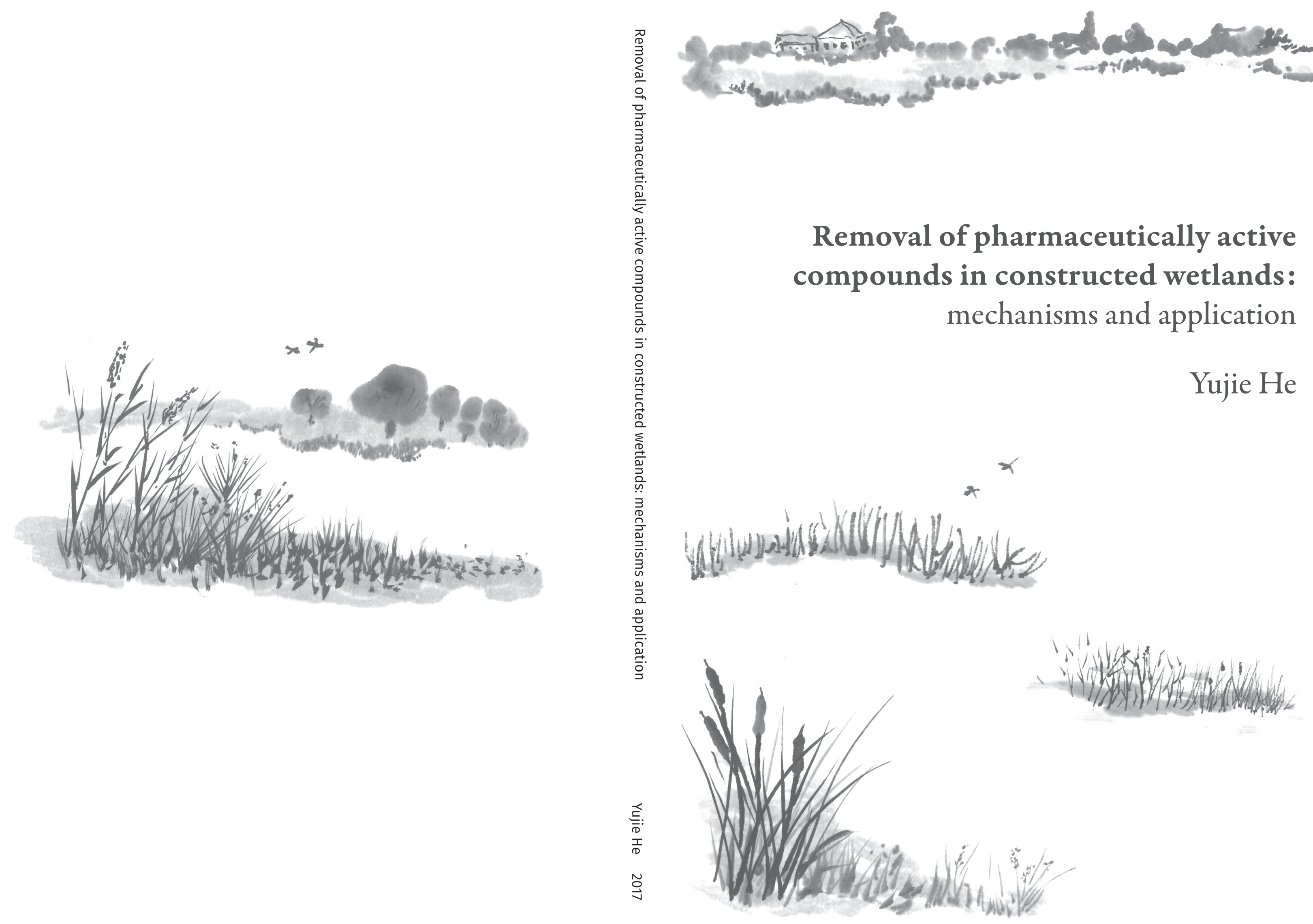


\section{Propositions}

1. A single technology cannot remove the suite of pharmaceuticals present in water. (this thesis)

2. "Green livers" are undervalued and insufficiently explored for water treatment, especially for pharmaceutical degradation.

(this thesis)

3. The process of reviewing and being reviewed is essential for acquiring the ability of scientific thinking.

4. Ignoring that humans are bound to lose control of their creations hinders technological and societal innovation.

5. Quality of life depends on availability and quality of food.

6. Policy makers can be much more effective at solving environmental problems than scientists.

7. Focusing on integrating limits one's integration into another culture.

Propositions belonging to the thesis, entitled

"Removal of pharmaceutically active compounds in constructed wetlands: mechanisms and application"

Yujie He

Wageningen, 10 October 2017 


\title{
Removal of pharmaceutically active compounds in constructed wetlands: \\ mechanisms and application
}

\author{
Yujie $\mathrm{He}$
}




\section{Thesis committee}

\section{Promotor}

Prof. Dr H.H.M. Rijnaarts

Professor of Environmental Technology

Wageningen University \& Research

\section{Co-promotors}

Dr A.A.M. Langenhoff

Associate professor, Sub-department of Environmental Technology

Wageningen University \& Research

Dr N.B. Sutton

Assistant Professor, Sub-department of Environmental Technology

Wageningen University \& Research

\section{Other members}

Prof. Dr R.H. Wijffels, Wageningen University \& Research

Prof. Dr R. Ji, Nanjing University, China

Prof. Dr W.P. de Voogt, University of Amsterdam

Prof. Dr P.J. van den Brink, Wageningen University \& Research

This research was conducted under the auspices of the Graduate School for Socio-Economic and Natural Sciences of the Environment (SENSE) 


\title{
Removal of pharmaceutically active
}

\section{compounds in constructed wetlands:}

\section{mechanisms and application}

\author{
Yujie He
}

\section{Thesis}

submitted in fulfilment of the requirements for the degree of doctor

at Wageningen University

by the authority of the Rector Magnificus,

Prof. Dr A.P.J. Mol,

in the presence of the

Thesis Committee appointed by the Academic Board

to be defended in public

on Tuesday 10 October 2017

at 4 p.m. in the Aula. 
Yujie He

Removal of pharmaceutically active compounds in constructed wetlands: mechanisms and application,

290 pages.

$\mathrm{PhD}$ thesis, Wageningen University, Wageningen, the Netherlands (2017)

With references, with summary in English

ISBN: 978-94-6343-632-8

DOI: $10.18174 / 419681$ 
To my beloved parents and brother

谨以此书献给我最爱的父母和弟弟 


\section{Contents}

Chapter 1

General introduction

Chapter 2

Evaluation of attenuation of pharmaceuticals, toxic potency, and antibiotic resistance genes in constructed wetlands treating wastewater effluents

Chapter 3

Degradation of pharmaceuticals in wastewater using immobilized $\mathrm{TiO}_{2}$ photocatalysis under simulated solar irradiation

\section{Chapter 4}

Influence of redox conditions on sorption and biodegradation of pharmaceuticals in sediment batch and column experiments

\section{Chapter 5}

Effects of dissolved organic matter and nitrification on biodegradation of pharmaceuticals in aerobic enrichment cultures

\section{Chapter 6}

Pharmaceutical biodegradation under three anaerobic redox conditions evaluated by chemical and toxicological analyses

\section{Chapter 7}

Metabolism of ibuprofen by Phragmites australis: uptake and phytodegradation

\section{Chapter 8}

Fate and distribution of pharmaceuticals in mesocosm constructed wetlands

\section{Chapter 9}

General discussion

Bibliography

Summary 
Chapter 1

General introduction 


\subsection{Pharmaceutically active compounds}

Pharmaceutically active compounds (PhACs) are a class of manmade chemicals to target metabolic and molecular pathways in humans and animals. PhACs encompass a diverse group of compounds, such as antibiotics, hormones, analgesic and anti-inflammatory, $\beta$-blockers, lipids regulator agents, and antiepileptic drugs [235]. PhACs have been extensively used in significant quantities on a global scale. For example, approximately 3000 different PhACs have been approved for human use in the European Union [329]. A large database study shows that the consumption of antibiotic drugs increased by $36 \%$ from 54 to 73 billion standard units between 2000 and 2010 [350]. The relative rates of production, release, and use of PhACs in the next 10 to 50 years is not easy to predict, but PhAC abundance in the environment is estimated to increase due to the increase of population, aging and affordability of medicines [208].

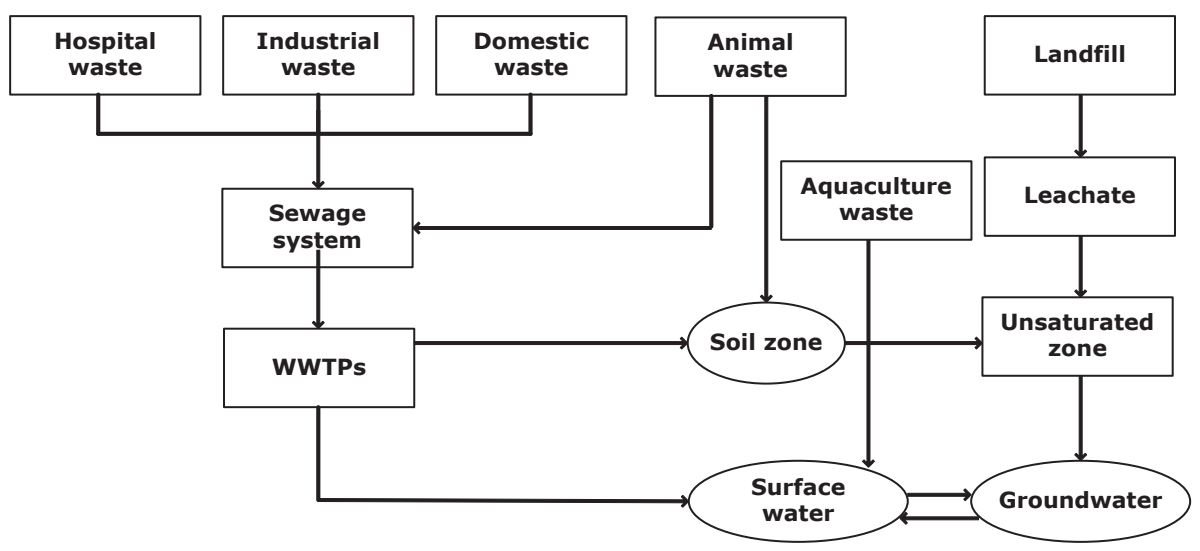

Figure 1.1 Sources and transportation pathways of PhACs in the environment [222]. Soil, surface water and groundwater are environmental acceptors of PhACs.

PhACs enter in the environment from various sources and transportation pathways (Figure 1.1). PhACs including their metabolites and conjugates are excreted in urine or faeces by humans or animals. Together with PhACs from hospitals and industries, PhACs in excrement enter wastewater treatment plants (WWTPs) where PhACs are partially degraded or sorbed to the activated or other sludge. The residual PhACs are discharged to the surface water while the sorbed PhACs can reach the terrestrial environment if the excess sludge is utilized as fertilizer in agriculture. PhACs could be released to surface water via aquaculture use. Additionally, PhACs could enter the terrestrial environment via animal manure excreted 
directly on the field or used in agriculture land, which can subsequently reach groundwater and surface water together with PhAC leachate from landfills. Presence of PhACs in wastewater effluent, surface water, groundwater and sediment has been extensively documented $[40,165]$.

Concentrations of PhACs in the environment are at trace-level ranging from $\mathrm{ng} / \mathrm{L}$ to $\mu \mathrm{g} / \mathrm{L}$ [165]. Still, PhACs could cause a significant cumulative effect on the metabolism of nontarget organisms and the ecosystems as a whole [32]. The earliest report of the adverse effect of PhACs on ecosystems was about the estrogenicity observed due to the release of endocrine disruptors. For example, exposure to $5 \mathrm{ng} / \mathrm{l}$ of ethinyloestradiol delayed embryonic development in zebrafish [199] and exposure at $0.1 \mathrm{ng} / 1$ induced vitellogenin in rainbow trout [297]. Drawn by the adverse effect of endocrine disruptors, Dutch research institutions implemented a joint project (LOES project; Landelijk Onderzoeko Estrogene Stoffen) to investigate the occurrence of those compounds in the aquatic environment in the Netherlands [278]. The LOES project reported that concentrations of endocrine disruptors are in the range of 0.3-150 ng/l in the wastewater and 0.3-7.2 $\mathrm{ng} / \mathrm{l}$ in the surface water. In 2004, an abnormal high death rate $(5-86 \%)$ of vulture in India and Pakistan was reported resulting from diclofenac, a widely used analgesic and anti-inflammatory PhAC [283]. In the study of Oaks et al. [283], a direct correlation between diclofenac exposure and renal failure of vultures was confirmed. Afterwards, antibiotics received public concern, as persistent exposure to antibiotics can cause the emergence of resistant bacteria strains [407]. It is believed that some bacteria naturally present in the environment are now carrying antibiotic resistance genes (ARGs) and may function as reservoirs of antibiotic resistance, which may be transferred to pathogenic bacteria and then may threaten human health in the coming decades or centuries [35].

Due to the cumulative effects of PhACs, regulatory agencies issue several PhACs on regulation lists in the past few years. The US Environmental Protection Agency placed one antibiotic and three hormones on the contaminant candidate list: erythromycin, estrone, estriol, and 17- $\beta$-estradiol (Drinking Water Contaminant Candidate List and Regulatory Determination, 2009, USA). Diclofenac, ethinylestradiol and 17- $\beta$-estradiol were included in the watch list of priority substance by the European Commission (Directive, 2013/39/EU, 2013). These regulations support the development of robust technologies to remove PhACs prior to their release into the environment. 


\subsection{Overview of treatment techniques}

WWTPs act as a key barrier against the PhAC disposal. However, conventional WWTPs are not designed for removing PhACs, but for removing bulk components such as sewage organic matter (hundreds to thousands mg chemical oxygen demand/l), and nutrients (tens to hundreds $\mathrm{mg}$ phosphorous or $\mathrm{mg}$ nitrogen/l). PhACs are present in this mixture at levels of $\mathrm{ng}$ to $\mu \mathrm{g} / \mathrm{l}$ and until recently ignored in optimizing WWTP. Therefore, discharge of WWTP effluents is currently a major pathway for the release of PhACs to surface water (Figure 1.1). An review revealed that PhACs were not sufficiently removed in WWTPs with 24 out of $50 \mathrm{PhACs}$ removed less than 50\% [359]. To polish up the insufficient removal, it is wise to establish post-treatment processes or optimize the existing post-treatment processes to further eliminate PhACs in WWTPs. In fact, with the identification and detection of PhACs in the environment, numerous post-treatment techniques have been exploited and explored for $\mathrm{PhAC}$ removal and can be categorized as physical, chemical, biological and hybrid techniques.

\subsubsection{Physical techniques}

As widely used physical techniques, membrane-based techniques, such as nano-filtration (NF) has the capacity to remove PhACs, whereas the pore sizes of micro- and ultra-filtration are much larger than the molecular size of PhACs [242]. It is reported that reverse osmosis (RO) has great potential to partially or significantly remove PhACs [388]. However, NF and RO membranes are permeable to some small PhACs [242]. Additionally, membrane-based techniques are high in energy demand and have continuous fouling issues [3].

Another type of physical technique is the use of activated carbon, applied as powder activated carbon (PAC) or granular activated carbon (GAC). PAC and GAC are considered as an effective adsorbent for removing persistent or non-biodegradable PhACs [3]. However, adsorption can only transfer PhACs from liquid phase to carbon phase without transformation and mainly work for hydrophobic compounds [331]. Furthermore, natural organic matter (NOM) in the wastewater would reduce the performance of PAC and GAC towards sorption of PhACs due to competitive adsorption of the NOM and blockage [162]. 


\subsubsection{Chemical techniques}

Disinfection techniques such as chlorination, ozonation and ferrate $\left(\mathrm{FeO}_{4}{ }^{2-}\right)$ were reported to oxidize PhACs to readily biodegradable compounds [3]. Ozonation was reported to significantly decrease PhACs as a post-treatment process at a full-scale WWTP [173]. Compared to chlorination, the reaction rate of ozonation was three orders of magnitude higher [303]. However, ozone production is energy intensive, which might increase the energy demand by $40-50 \%$ in a WWTP [3]. Additionally, ozonation performance is sometimes unstable resulting from the interference of radical scavengers [244]. Ferrate is a powerful disinfection agent by oxidizing contaminants with $\mathrm{Fe}^{6+}$ and coagulating contaminants with $\mathrm{Fe}^{3+}$ [187]. It is reported that ferrate can oxidize up to $90 \%$ of $\mathrm{PhACs}$ at $\mathrm{ng} / \mathrm{l}$ level. Nevertheless, the ferrate ions have a poor stability in the liquid phase and manufacturing is costly [3].

Fenton-based techniques are well known for the oxidization of water-miscible organic contaminants by $\bullet \mathrm{OH}$ produced by the reaction of $\mathrm{H}_{2} \mathrm{O}_{2}$ and $\mathrm{Fe}^{2+}$ [238]. Fenton oxidation can even improve the biodegradability of PhAC in wastewater [342]. However, iron sludge is produced during the Fenton oxidation due to the precipitation of $\mathrm{Fe}(\mathrm{OH})_{3}$, particularly at high $\mathrm{pH}$, which requires to be treated and disposed properly [348]. To reduce the production of iron sludge, Fenton process was modified to electro-Fenton and photo-Fenton by introducing electricity and light to reduce the consumption of catalytic iron [3]. The modified Fenton techniques can efficiently oxidize PhACs, but as with conventional Fenton, the performance is strongly dependent on the $\mathrm{pH}$ of the aqueous solution and the dosage of $\mathrm{H}_{2} \mathrm{O}_{2}$ and $\mathrm{Fe}^{2+}$ [328]. Additionally, Fenton-based techniques are less efficient when treating hydrophobic organic contaminants with a high octanol-water partition coefficient $\mathrm{K}_{\mathrm{ow}}[3]$.

Photolysis is a process in which PhAC molecules can be decomposed by absorbing light directly or absorbing irradiation indirectly. The indirect photolysis occurs in the presence of natural photo-sensitizers such as dissolved $\mathrm{NOM}, \mathrm{Cl}^{-}, \mathrm{NO}_{3}{ }^{-}, \mathrm{Fe}^{3+}$ and manually added photosensitizers such as $\mathrm{H}_{2} \mathrm{O}_{2}[3,387]$. Different light sources are explored to simulate photolysis. To date, UV light is now most commonly used; higher energy electromagnetic radiations such as x-ray and gamma radiation are intensively investigated recently. Moreover, sunlight induced photolysis draws more and more attention due to the high cost of artificial light application $[3,306]$. 


\section{General introduction}

To enhance photolysis efficiency, photocatalysis was proposed in which transformation of PhACs can be stimulated by the catalyst [86]. Semiconductor metal oxides such as $\mathrm{TiO}_{2}$ and $\mathrm{ZnO}$ are mostly used [233]. The sunlight induced photocatalysis for $\mathrm{PhAC}$ removal is currently still under research [138].

\subsubsection{Biological techniques}

The membrane bioreactor (MBR) is widely considered as a state of the art technique for $\mathrm{PhAC}$ removal [3]. MBRs can remove a wide range of PhACs as ascribed mainly to the long sludge retention time, allowing the sludge to adapt to micropollutant degradation, and partially to physical interception of PhACs [242]. Thus, MBR techniques combine activated sludge treatment and membrane filtration. Nevertheless, membrane fouling, high energy and operational costs, and high cost of membranes are main obstacles for wide application of MBRs [271].

Attached growth techniques were reported promising to treat contaminants by cultivating microorganisms on the inert carriers either fixed or mobilized in the suspension of reactors [242]. Commonly used attached growth techniques are trickling filter, sand filtration and biological activated carbon (BAC) filtration. Recently, the attached growth concept has been extended for PhAC removal by applying the fixed bed biofilm reactor [130] and moving bed biofilm reactor [1]. Additionally, more economical bio-plastic materials have been investigated such as granules and pellets to replace the commonly used Kaldnes-like carriers for scaling up application in the future [1].

Apart from microorganisms-based biological treatment techniques, other microorganisms such as algae and fungi can also attenuate PhACs, often in a cost efficient and sustainable way. For example, a high-rate algal pond takes advantage of both microalgae and other microorganisms to biodegrade PhACs. In the algal pond, PhACs are degraded by heterotrophic microorganisms which consume oxygen produced by microalgae photosynthesis and thereby no aeration is required [267]. Additionally, the microalgae-based technique gains popularity due to the resource recovery of the algal biomass into fertilizer and biofuel [3]. White-rot fungi can produce intracellular (e.g. cytochrome P450) and extracellular (e.g. laccases, peroxidases) enzymes [137]. Those enzymes with low substance specificity can attack PhACs by acting as biocatalysts [250]. To date, limited research is available for PhAC bioremediation by using those microorganism-based techniques. 
Phytoremediation is a technique that uses plants (crop and wetland plant species) and associated rhizosphere microorganisms to remove, transform, and detoxify contaminants [59, 339]. These green techniques are often characterised as sustainable techniques with low energy and manpower input and being effective in numerous cases for treating contaminants including PhACs [206, 322]. However, accumulation of contaminants in the plant biomass might result in phytotoxicity [139], which can diminish or prevent plant growth in the field and thus negatively impact on phytoremediation.

\subsubsection{Hybrid techniques}

Biological techniques are by far the most widely used for removal of emerging contaminants including PhACs [3]. Meanwhile, physical and chemical techniques are generally considered to be also necessary, i.e. to treat non-biodegradable PhACs or increase their biodegradability. Hybrid techniques combine physical, chemical, and biological methods. Considering the complicated sources of PhACs (Figure 1.1), resulting in a cocktail of compounds, it may be required to develop such hybrid techniques to achieve high removal of a wide range of PhACs. Indeed, hybrid techniques showed promising removal performance for PhACs. For instance, the combination of ozonation and biological enhanced AC removed 94-99\% of analgesics pharmaceuticals such as carbamazepine, codeine, diclofenac, ibuprofen, naproxen, paracetamol and tramadol [3]. The combination of MBR and RO removed 83-100\% of sotalol, salbutamol, salicylic acid, and beta blockers [3]. An algae photo-reactor removed $60-100 \%$ of diclofenac, ibuprofen, paracetamol and metoprolol by exerting the combination of biodegradation and photolysis [92]. In general, sufficient PhAC removal can be achieved in the hybrid techniques by appling material/energy-intense physical and chemical techniques. However, considering the costly construction and maintenance of most of these techniques, more cost-efficient and sustainable hybrid techniques are required for long-term $\mathrm{PhAC}$ removing post-treatment. Wetland systems may be attractive for this purpose.

Wetland systems are based on shallow ponds, beds, or trenches that contain floating or emergent rooted wetland vegetation [137], which are normally categorized as a cost-effective biological technique. A wetland can be constructed to efficiently treat wastewater by various processes including photodegradation, sorption, (aerobic/anaerobic) biodegradation, and phytoremediation [224]. In principle, a constructed wetland (CW) itself can be considered as a "hybrid" system. In addition, PhACs in the environment are naturally attenuated by the processes identical with in CWs. Therefore, $\mathrm{CW}$ is actually an integrated and enhanced 


\section{General introduction}

version of natural ecosystem for PhAC removal. Furthermore, the main $\mathrm{PhAC}$ acceptors in the environment include soil/sediment, water, and plants, which are also assembled in CWs. This makes the investigation of $\mathrm{PhAC}$ removal in $\mathrm{CWs}$ even more worthwhile, because fate and distribution of PhACs in the environment can be further understood.

\subsection{Removal of PhACs in CWs}

CWs are managed as water quality improving systems, owing to their low cost, ease of operation/maintenance, and environmental-friendliness and sustainability. CWs have been documented to remove various contaminants such as organic carbon, suspended solids and nutrients since the 1970s [140, 400]. With the increasing concern of PhACs, CW potential for eliminating PhACs has been increasingly investigated in recent years.
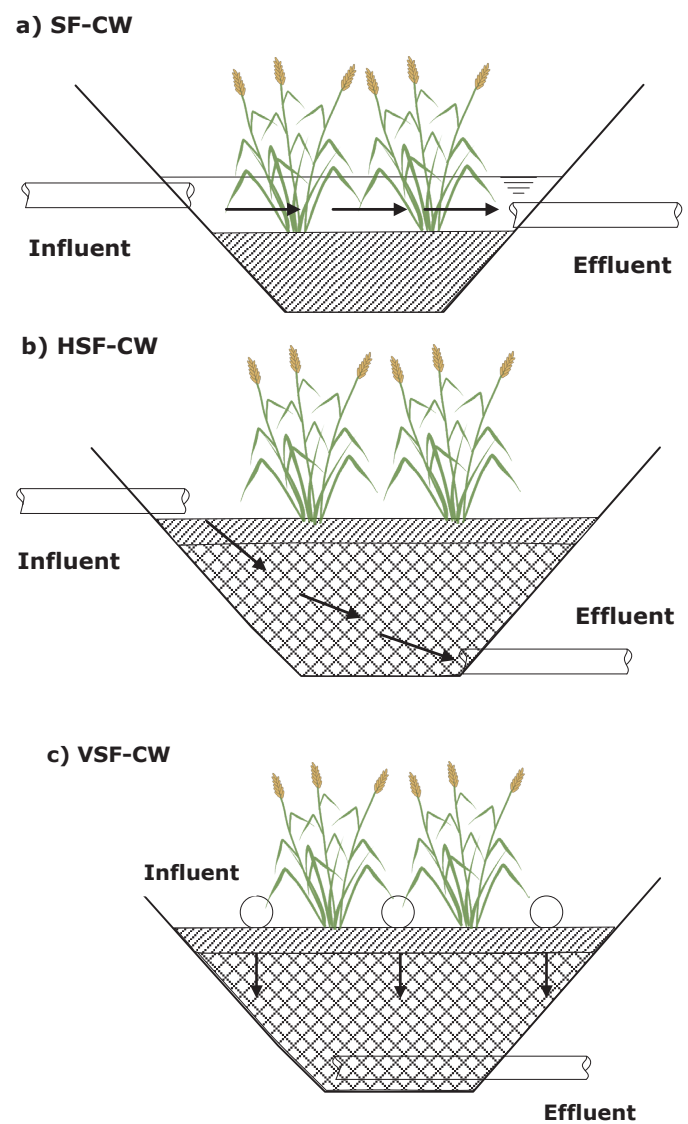

Figure 1.2 Structure of different configurations of $\mathrm{CWs}$ a) surface free water $\mathrm{CW}$ (SF-CW); b) horizontal subsurface flow CW (HSF-CW); c) vertical subsurface flow CW (VSF-CW). 


\subsubsection{Removal performance}

CWs applied as a post-treatment process can be categorized into free water surface flow CWs (SF-CWs), horizontal subsurface flow CWs (HSF-CWs), vertical subsurface flow CWs (VSFCWs), and a combination of CWs with these different configuration (Figure 1.2). In SF-CWs, free water flows at relatively shallow depth over an impermeable bottom liner or packed substrate layer. In HSF-CWs, water flows horizontally through the subsurface of wetland bed where substrate was packed. In VSF-CWs, water was distributed evenly onto the surface of the wetland bed and then flows vertically from the planted later down through the substrate layer. Removal of PhACs from wastewater effluent in pilot-scale or full-scale of CWs with different configurations is summarized in Table 1.1. In summary, CWs can provide promising $\mathrm{PhAC}$ removal performance but the removal varies in different conditions.

Removal efficiencies of PhACs in CWs can be affected by many factors [378]. These include design parameters (configuration types, vegetation, substrate matrix, depth), operational parameters (hydraulic retention time HRT, feeding mode) and environmental factors (redox potential, temperature), as partially reflected in Table 1.1. Taking the configuration type as an example, SF-CWs commonly include ponds, lagoons and free water basins [362]. This configuration makes SF-CWs the most popular systems for wastewater post-treatment compared to the other types due to their larger storage capacity and more natural landscape effects [400]. In comparison, HSF-CWs and VSF-CWs possess a superior rhizosphere effect and more sorption surface in the roots zone due to the subsurface flow, which may enhance PhAC removal. On the other hand, subsurface flow increases the construction and maintenance cost $[119,400]$.

$\mathrm{PhAC}$ elimination in CWs is a result of complex processes, mainly photodegradation, sorption, biodegradation, and phytoremediation. These removal processes are the functional tools of the $\mathrm{CW}$ system. Only if we understand how the different tools work, optimal performance can be achieved by applying them in an optimal combination. So far, relevant studies mainly focus on removal efficiencies of PhACs in full-scale CWs, comparison of different types of CWs in removing $\mathrm{PhACs}$ by lab-scale experiments, or application of $\mathrm{CWs}$ in removing $\mathrm{PhACs}$ from different sources of wastewater. However, there are fewer studies investigating mechanisms of the individual processes, i.e. individual tools, for PhAC removal in CWs. 
General introduction

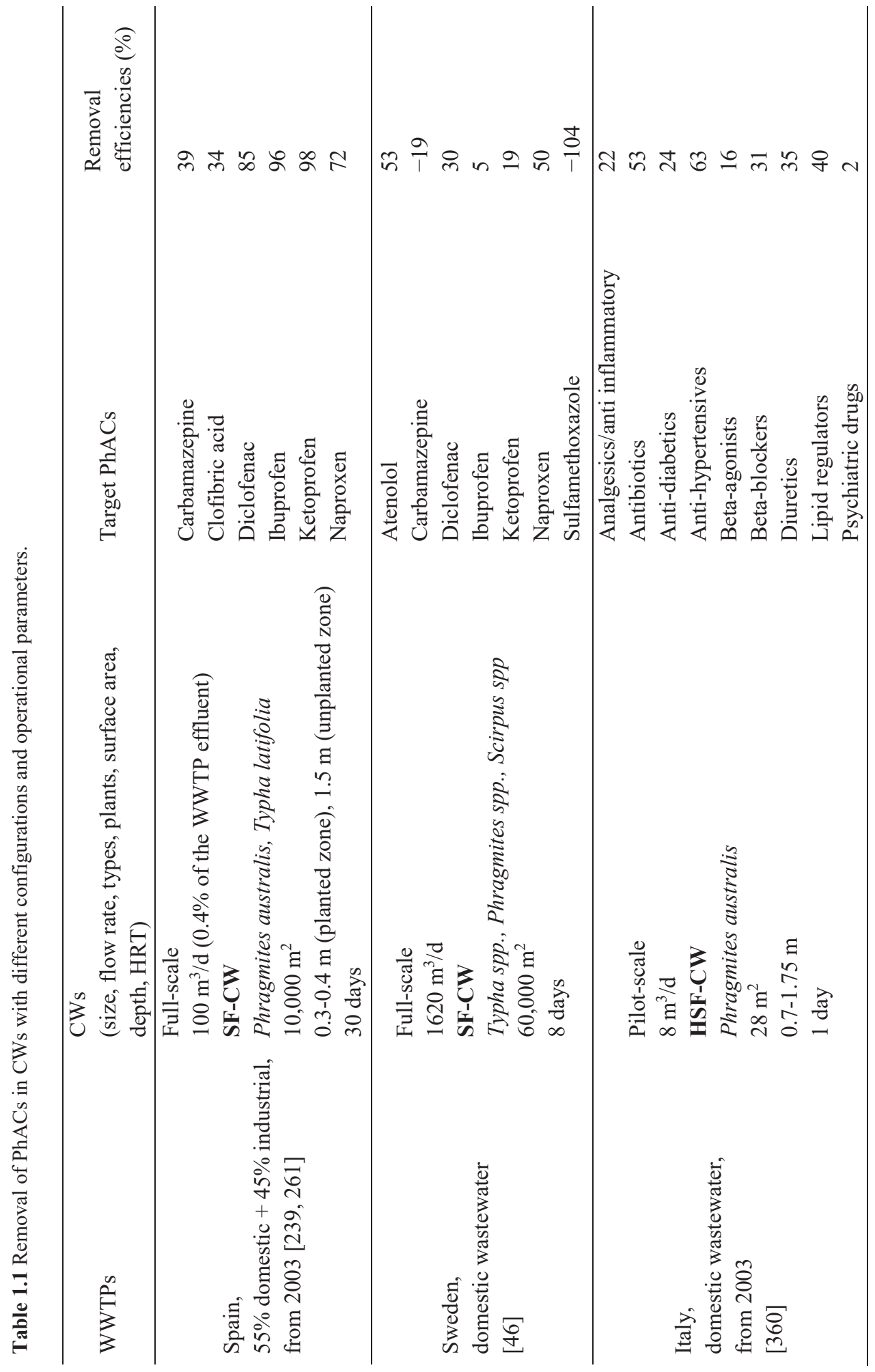


Chapter 1

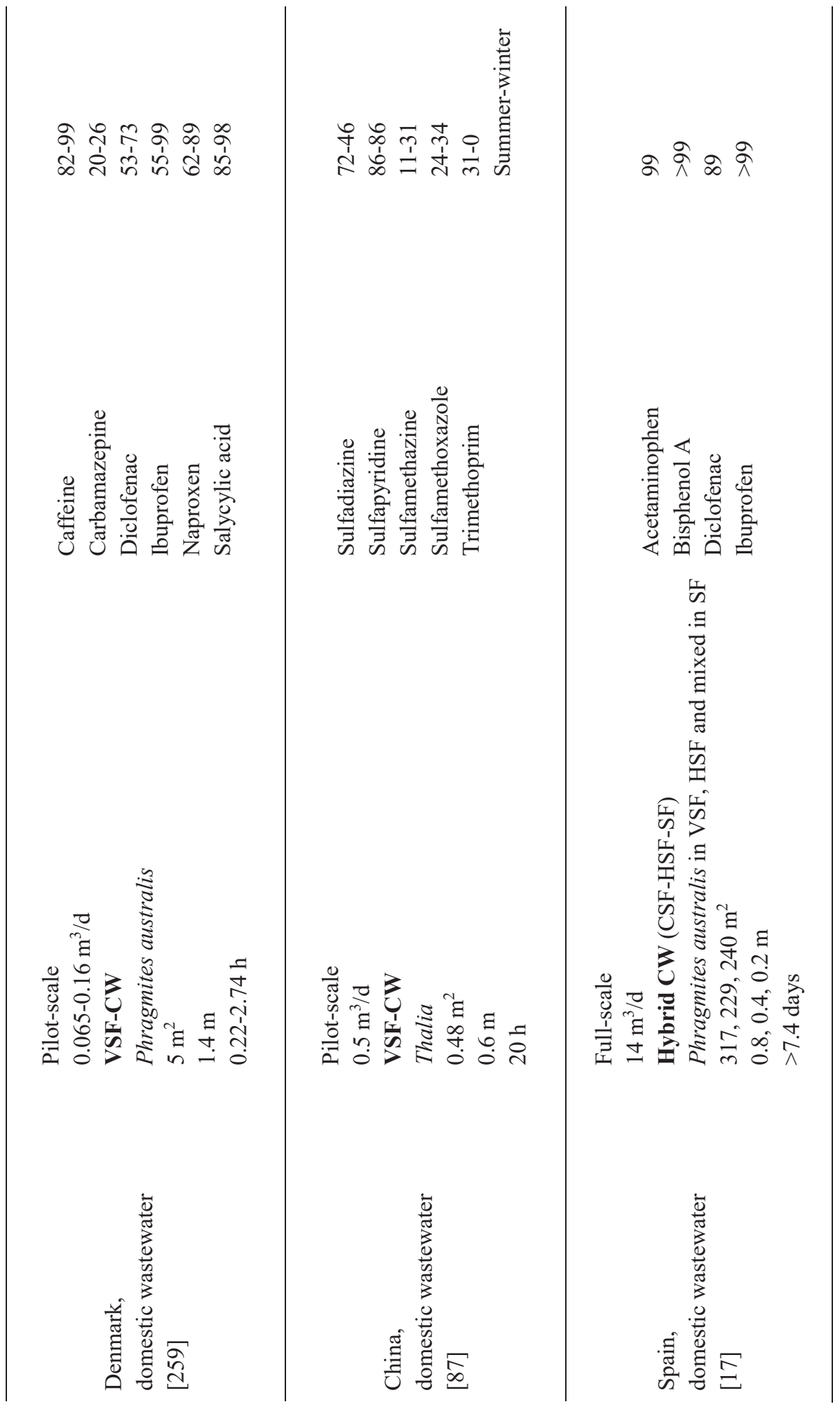




\section{General introduction}

\subsubsection{Removal processes}

CWs appear to have a promising future for treating PhACs as the tertiary treatment process. Nevertheless, they are generally viewed and investigated as a "black box" due to the complex interplay of the removal processes [400], as shown in Figure 1.3.

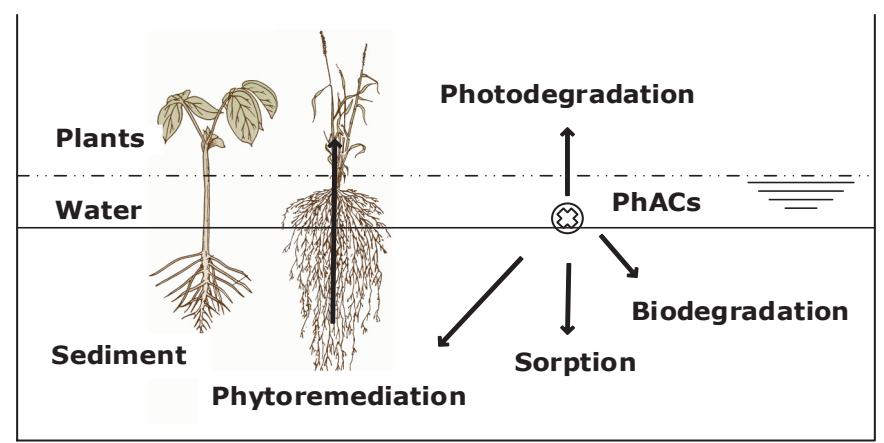

Figure 1.3 The main removal processes of PhACs in the $\mathrm{CW}$, including photodegradation, sorption, biodegradation, and phytoremediation.

Photodegradation is suggested to be a significant and predominant removal process for PhACs in a CW [10]. PhACs can be degraded by direct and/or indirect photolysis. Several factors affect photodegradation efficiency in aquatic systems, including seasonal variation (sunlight availability), light intensity, and light attenuation by water depth [261]. Nonetheless, photodegradation of PhACs are more intensively studied in surface water bodies such as lakes, rivers, and sea. Thus far, there are only limited numbers of studies about understanding the photodegradation process of PhACs in field CWs [400].

PhACs can be sorbed by the CW substrate, including soil, organic carbon, mineral surface and biofilms coating the gravel bed [400]. Apart from the substrate originally in a CW, suspended particles and organic carbon from wastewater effluent can be retained in the CW bed and further sorb PhACs [257]. Sorption performance can be influenced by physicochemical properties of PhACs, composition of soil NOM, and environmental factors such as $\mathrm{pH}$, temperature, and ionic strength $[48,80]$. For example, hydrophobic PhACs have great potential to be sorbed compared to hydrophilic PhACs. However, at present very few data are available on understanding the sorption behaviour of PhACs in the $\mathrm{CW}$ sediment, compared to the well-investigated PhAC sorption in soil and river sediment $[97,251,300]$. 
The potential of PhACs to undergo biotransformation is an important aspect for their removal process. PhAC biodegradation in CWs could occur under both aerobic and anaerobic conditions by various microorganisms such as heterotrophic microorganisms, autotrophic microorganisms, fungi (basidiomycetes and yeasts), and specific protozoa [191]. Biodegradation of PhACs is influenced by the physicochemical properties of PhACs and environmental parameters, such as redox conditions, composition and activity of the microbial communities [311]. Previous studies mainly report the appearance of parent PhACs, while much less is known for the formation of intermediates that may also be persistent and even more toxic than the parent compounds [265, 397].

Direct uptake and translocation of PhACs by plants have been considered as important mechanisms for phytoremediation [79, 103]. It is reported that the uptake and translocation process could be simply driven by diffusion and no specific transporters exist in plant cells to transfer PhACs into plant tissues [105]. Meanwhile, there is a statement about phytodegradation that, following uptake and translocation PhACs might be transformed to less toxic compounds in plants [312]. To date, phytoremediation of PhACs is mainly studied in hydroponic systems rather than in the real substrate system. Additionally, the phytoremediation significance is mainly proved indirectly by mass balance rather than by direct analytical detection of PhACs in plant tissues $[102,265]$.

\subsection{Research opportunities}

CWs have a mixture of actors (water, sediment, microorganisms, and plants) involved in complicated conversion processes. There are thus a large number of research opportunities available that need to be addressed to enable the understanding of $\mathrm{CW}$ systems and to improve their application. The following knowledge demands are emerging for research on $\mathrm{PhAC}$ removal by CWs:

1) To comprehensively evaluate $\mathrm{CW}$ performance on removal of $\mathrm{PhACs}$ and $\mathrm{PhAC}$-related hazards.

Performance for PhAC removal is normally evaluated based on chemical analysis of $\mathrm{PhACs}$ in most studies. However, it is nearly impossible to identify not only all $\mathrm{PhACs}$ but also the intermediates in complex wastewater matrices at low concentrations. Furthermore, chemical analysis alone gives limited information to understand the potential hazards of PhACs and their intermediates on the aquatic environment [349], such as toxicity to non-target organisms 


\section{General introduction}

and acting as selection pressure of antibiotic resistance as described in section 1.1. Bioassays employing different test organisms can directly characterize the toxicity levels of known and unknown components in a mixed wastewater matrix [391]. ARGs can be qualified by molecular analysis techniques. Under the assistance of statistical analysis tools, outcomes of chemical, toxicological, and molecular analyses could be combined to explore the correlation of PhACs and related hazards. Such combined studies for CWs are limited and no clear correlation has been established between these analyses.

2) To understand the individual processes and their interaction.

Among the individual removal processes (Figure 1.3), the underlying mechanism of phytoremediation in CWs is much less known. Some research have identified that PhACs can be taken up and translocated in wetland plant species by mass balance calculation $[102,104$, 206, 409]. Moreover, whether the plants can phytodegrade PhACs after uptake and translocation is not clear. To date, only two studies reported phytodegradation of diclofenac and carbamazepine in wetland plant species by detecting intermediates in the tissues [27, 104]. Plants appear to contain isoenzymes with the specificity to detoxify xenobiotics, which resembles mammalian livers [314]. The potential PhAC transformation in plants might be explained by the interactions with PhACs and the associated enzymes. Therefore, more research needs to be implemented to quantitatively evaluate the $\mathrm{PhAC}$ phytoremediation including uptake, translocation, and phytodegradation and to explore the underlying mechanism of phytodegradation.

The individual removal processes in CWs could influence each other. For example, in CWs plant can create conditions favourable for $\mathrm{PhAC}$ removal via biodegradation and sorption. Plants in CWs can stabilize the substrate bed and act as anchoring surface for biofilm. Moreover, roots of plants can release $\mathrm{O}_{2}$ and exudates to the rhizosphere which could stimulate microbial growth and microbial activity [137]. It is reported that enzymes released from roots can interact with and/or even mineralize $\mathrm{PhACs}$ to compounds such as $\mathrm{CO}_{2}, \mathrm{H}_{2} \mathrm{O}$, $\mathrm{Cl}_{2}$ [269]. The released exudates contain ions, inorganic and organic acids, proteins, and enzymes [313], which may affect the sorption capacity of sediment as well. Therefore, in addition to understand the individual processes, it is of importance to understand the interactions between the processes related to $\mathrm{PhAC}$ removal in CWs. 
3) To understand the effect of important environmental factors on PhAC removal.

Environmental factors such as NOM, redox conditions, temperature, and $\mathrm{pH}$ are relevant for PhAC removal [272, 400]. NOM is a heterogeneous mixture containing molecules with different sizes, structures, functions, and properties, which can affect environmental behaviour of organic contaminants such as their solubility, bioavailability, and transport [66]. NOM, including dissolved NOM and solid NOM, originates in CWs from wastewater effluent, dead plant tissues, and the NOM originally contained in the substrate. NOM is an important environmental factor as it may affect photodegradation, sorption, and biodegradation of PhACs in CWs. During photodegradation, dissolved NOM could enhance the transformation of PhACs by acting as a photosensitizer [51], especially for organic contaminants with a phenolic OH group [364]. On the other hand, dissolved NOM could inhibit the photodegradation by quenching reactive species such as $\bullet \mathrm{OH}$ and screening photochemically active light [387]. During sorption, NOM can provide readily accessible sites and rate-limited sites to organic contaminants depending on the properties of the contaminants and composition of the NOM $[250,402]$. During biodegradation, NOM can affect the process by acting as a) electron acceptors for microbial respiration; b) electron donors to reduce more oxidized electron acceptors and compete with PhACs; and c) electron shuttles between electron donors and electron acceptors and thus enhance redox reactions [253]. Therefore, it is of great interests to investigate how NOM affects the fate of PhACs in CWs.

Different redox conditions take place simultaneously in CWs, such as aerobic, nitrate reducing, sulfate reducing, and methanogenic conditions [334]. The ability of microorganisms in CWs to biodegrade a wide spectrum of PhACs can be ascribed to the coexistence of aerobic and anaerobic micro-environments [362]. Nitrification is a reaction that occurs under aerobic conditions, and has been reported to play a significant role in $\mathrm{PhAC}$ biodegradation by co-metabolism. Ammonia mono-oxygenases (AMO) of ammoniaoxidizing bacteria is believed to be responsible for co-metabolizing PhACs resulting from its broad substance specificity [89]. For example, a previous study concluded that biotransformation of ethinylestradiol can be mediated co-metabolically by AMO [390]. However, several studies also concluded that AMO is ineffective for biodegradation of erythromycin, roxithromycin, and fluoxetine in nitrifying activated sludge [121]. Thus, the role of nitrification in $\mathrm{PhAC}$ biodegradation still needs further clarification, especially for $\mathrm{PhACs}$ with different physicochemical properties. Anaerobic biodegradation of PhACs are typically studied under general anaerobic conditions without distinguishing specific electron 


\section{General introduction}

acceptors, such as nitrate, sulfate, and $\mathrm{CO}_{2}$. Therefore, knowledge on $\mathrm{PhAC}$ biodegradation dependent on specific anaerobic redox conditions need to be further explored.

4) To enhance photodegradation and biodegradation of PhACs in CWs.

Capacity of CWs towards $\mathrm{PhAC}$ removal can be enhanced through strengthening the individual removal processes. Especially, photodegradation and biodegradation are typically the most influential processes for degradation of organic micropollutants in WWTP effluentreceiving waters [362]. There are a few studies on PhAC photodegradation in natural plantbased systems like CWs, compared to related studies in surface water bodies such as lakes, rivers, and seas. One of the reasons is presumably due to the high plant coverage in natural plant-based systems, which blocks potential sunlight irradiation. Thus, an open shallow unit constructed prior to the wetland can be considered as a strategy for photodegradation enhancement. In addition, photocatalysis under sunlight irradiation can be considered to maximize the photodegradation performance. Other added values of enhancing photodegradation are to increase the biodegradability of contaminants and to detoxify the wastewater streams [284]. In terms of biodegradation enhancement, a primary way is to acclimate and enrich the microbial community for removing PhACs. By exposing microorganisms in growth medium containing PhACs, microorganisms may regulate their metabolism toward using PhACs as the carbon source and/or electrons for energy and growth, or toward tolerating the stress resulting from PhACs by changing their own morphology and/or physiology [325]. Therefore, microbial enrichment could be a promising strategy to increase the biodegradation capacity for PhAC removal.

5) To explore the fate and distribution of different PhACs in CWs.

Literature on PhACs in CWs handles the system as a black box, mostly focusing on measuring influent and effluent concentrations of PhACs to evaluate the overall removal performance. These studies generally do not investigate the fate of PhACs during removal and their distribution in the matrices of CWs. For PhACs with different physicochemical properties, their removal mechanisms in real CWs via photodegradation, sorption, biodegradation, and phytoremediation are rarely reported. To date, there are several studies evaluating the photodegradation, biodegradation, and phytoremediation of $\mathrm{PhACs}$ in hydroponic mesocosm CWs [265, 397]. By comparing removal efficiencies of PhACs between varying designed mesocosm $\mathrm{CWs}$, the authors concluded the relative contribution of individual processes. For example, biodegradation was ascribed to the removal of naproxen 
and ibuprofen while diclofenac was mainly removed by photodegradation. However, in the real CWs with sediment, the conclusions might become different as microorganisms and components (e.g. NOM) in the substrate will involve sorption, biodegradation, and even photodegradation as explained previously. Nowadays, it is possible to assess the distribution of PhACs in CWs by direct measurement of PhACs in different matrices (surface water, wastewater, sediment, plant tissues) due to the development of extraction and analytical methods $[27,143,240]$. Therefore, it is essential and feasible to study the fate and distribution of PhACs with different physicochemical properties in substrate-based CWs via direct measurement.

\subsection{Research objectives}

As described above, the cost-effective and environmental-friendly technique $\mathrm{CW}$ has a promising potential to remove $\mathrm{PhACs}$ as a post-treatment process. A $\mathrm{CW}$ itself can be considered as a "hybrid" system containing photodegradation, sorption, biodegradation, and phytoremediation processes. Those removal processes are the efficient tools of the $\mathrm{CW}$ system. Nevertheless, CW has been often viewed as a "black box" due to the complicated interplay of the removal processes. Therefore, it is of importance to obtain more knowledge to transform the black box into a transparent understanding of the individual tools and to take advantage of that to make tailored, controllable, and optimisable $\mathrm{CW}$ for $\mathrm{PhAC}$ removal for specific situations. In addition, PhACs in the environment are naturally attenuated by the same processes as in CWs. CW is actually an integrated and enhanced version of natural ecosystem in which PhACs can be transported and removed. The main PhAC acceptors in the environment including soil/sediment, water, and plants are assembled in CWs. Thus, investigating the fate and distribution of $\mathrm{PhACs}$ in a $\mathrm{CW}$ is in principle investigating $\mathrm{PhAC}$ behaviour in the natural environment.

Linked to the described research opportunities, the overall objectives of this research are to:

1) evaluate the attenuation capacity of field CWs for removal of PhACs and PhACrelated hazards (Chapter 2);

2) understand mechanisms of individual processes, including photodegradation (Chapter 3), sorption (Chapter 4), biodegradation (Chapter 4, 5, and 6) and phytoremediation (Chapter 7), and the interactions between them in CWs (Chapter 8); 


\section{General introduction}

3) investigate the effect of important environmental factors (i.e. NOM, redox conditions) on the removal processes (Chapter 3, 4, 5, 6, and 8);

4) enhance photodegradation and biodegradation capacity in CWs (Chapter 3, 5, 6, and 8);

5) understand the fate and distribution of PhACs in CWs (Chapter 8).

\subsection{Outline of the thesis}

The overview of the thesis outline to meet the research objectives and answer the research questions is provided in Figure 1.4.

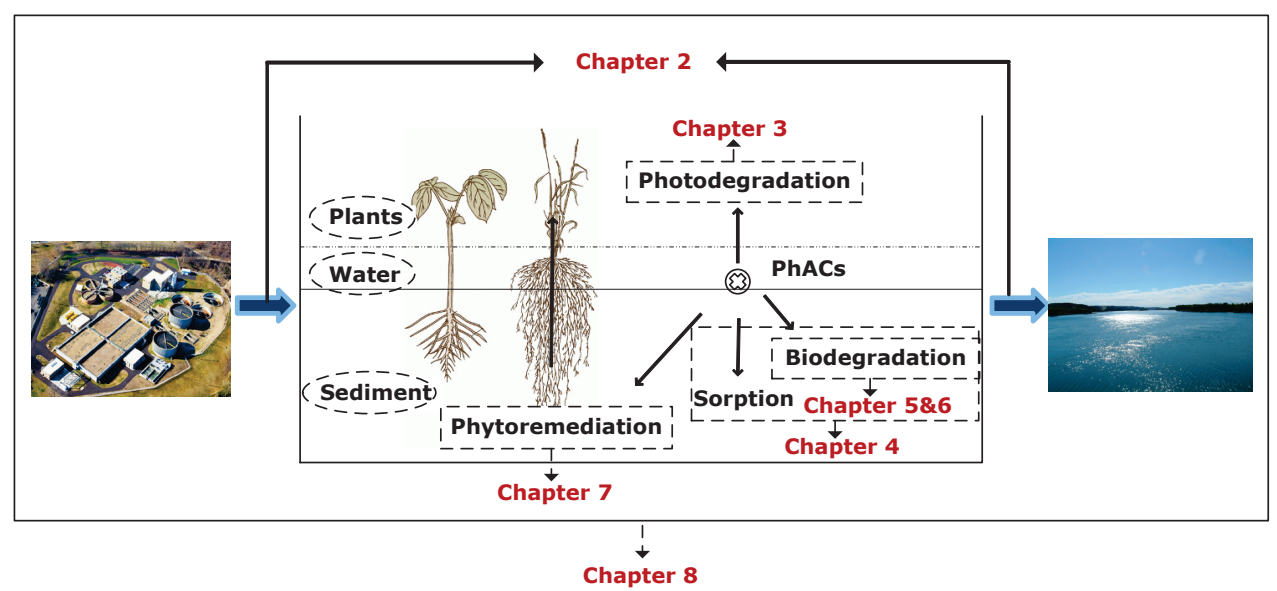

Figure 1.4 Schematic outline of this thesis. Chapter 2 focuses on the occurrence and removal of PhACs in field CWs, Chapter 3-7 focus on individual processes and optimization of photodegradation and biodegradation (Chapter 3-photodegradation, 4-sorption and biodegradation, 5-aerobic biodegradation, 6-anaerobic biodegradation, 7-phytoremediation), Chapter 8 focuses on the fate and distribution of $\mathrm{PhACs}$ in a whole $\mathrm{CW}$, where enhancement of individual removal processes were also merged.

Chapter 2 describes the performance of PhAC removal in existing field CWs as the posttreatment process for removing bulk components. Wastewater samples were collected from three CWs containing two SF-CWs and one VSF-CW. The occurrence of PhACs in the CW influents was screened for the most abundant PhACs, which were used as the target compounds for further lab-scale studies. Attenuation of PhACs, toxic potency, and ARGs was assessed in the CWs and their correlations were analysed by statistical tools. Removal performance of CWs with different configuration types and operational parameters was 
compared to delineate the significant removal processes in CWs, which was further optimized in the following chapters.

Chapter 3 demonstrates the effect of solar irradiation on a PhAC mixture in wastewater and the potential of a photocatalysis technique to enhance photodegradation in CWs. In a batch experiment, the catalyst $\mathrm{TiO}_{2}$ was immobilized on sand and Xenon light was employed to simulate solar light. Photolysis and photocatalysis of a PhAC mixture were compared under four days irradiation, which is a typical HRT applied in CWs. Irradiation of PhACs in wastewater effluent and deionized water were compared to identify the role of dissolved NOM during photodegradation. From an application perspective, the importance of water depth in photodegradation was determined by modelling and experiments. Toxicity and biodegradability of $\mathrm{PhAC}$ mixture was measured to evaluate the application potential of the photocatalysis technique.

Chapter 4 illustrates the sorption and biodegradation of a PhAC mixture in $\mathrm{CW}$ sediments by using batch and column set-ups. The sorption and biodegradation behaviour of individual PhACs were compared to identify the difference between PhACs with different physicochemical properties. In addition, the effect of specific redox conditions on the removal of PhACs in sediment microbial populations were assessed in both set-ups by applying aerobic, nitrate reducing, sulfate reducing, and methanogenic conditions. Enrichment was tested under aerobic conditions to determine its feasibility to enhance the biodegradation capacity of sediments for PhAC removal.

Chapter 5 presents the effect of dissolved NOM and nitrification on the biodegradation of a $\mathrm{PhAC}$ mixture by an enrichment culture. The enrichment culture applied in the batch experiment originates from the natural aerobic sediment inoculum, enriched in the work described in Chapter 4. Dissolved NOM extracted from the natural CW sediment was added to the batches to examine the role of dissolved NOM in PhAC biodegradation. Nitrification, a prevalent redox reaction under aerobic conditions, was investigated to understand its effect on the biodegradation of PhACs.

Chapter 6 displays the biodegradation of a PhAC mixture by an enrichment culture under different anaerobic redox conditions. The enriched culture applied in the batch experiment is from the anaerobic sediment culture enriched in Chapter 4. PhAC biodegradation under nitrate reducing, sulfate reducing, and methanogenic conditions were investigated by looking at the removal efficiencies of parent PhACs as well as the production of intermediates. 


\section{General introduction}

Biodegradation of PhACs as individual compounds or as a mixture of PhACs was compared to understand the interactions between PhACs. The biodegradation process was evaluated by a combination of chemical and toxicological analyses. Additionally, correlations were identified between chemical and toxicological data by statistical tools by which more knowledge of the biodegradation process can be explored.

Chapter 7 depicts the uptake, translocation, and transformation of ibuprofen in a widely applied wetland species Phragmites australis. The batch experiment was conducted in perlite pots under greenhouse conditions. Plants treated with ibuprofen were harvested to extract ibuprofen and potential intermediates in the roots and rhizomes (RR) tissue, and the tissue of stems and leaves (SL). Ibuprofen and potential intermediates were measured in the RR, SL, and in the liquid medium to evaluate the uptake, translocation, and transformation of ibuprofen. Treated and untreated plants were compared to determine the phytotoxicity of ibuprofen and to identify the variation of enzymatic activity during exposure. By correlating the behaviours of ibuprofen and enzymatic activity in the plants, the underlying mechanism of ibuprofen uptake and transformation in the plants were addressed.

Chapter 8 demonstrates the fate and distribution of PhACs in CWs, and the role of individual removal processes in CWs. Mesocosm CWs were operated for 4 months to treat wastewater spiking with continuous PhAC streams. Levels of PhACs and ibuprofen intermediates were measured in liquid aqueous, substrate (mainly sediment), and plants to estimate the mass balance of PhACs in the mesocosm CWs. Dominant removal processes were identified for individual PhACs with different physicochemical properties. By comparing CWs with different conditions, effect of irradiation via pre-photocatalysis, substrate composition through litter (dead plant biomass, solid NOM) addition, and plants were investigated on PhAC removal. Accordingly, recommendations of enhancing $\mathrm{CW}$ capacity for PhAC removal were proposed.

Finally in Chapter 9, findings from previous chapters were integrated to highlight the potential of $\mathrm{CW}$ application for $\mathrm{PhAC}$ removal, summarize removal mechanisms of $\mathrm{PhACs}$ in CWs, and propose feasible strategies for enhancing capacity of CWs. Furthermore, future perspectives on research and application were discussed. 


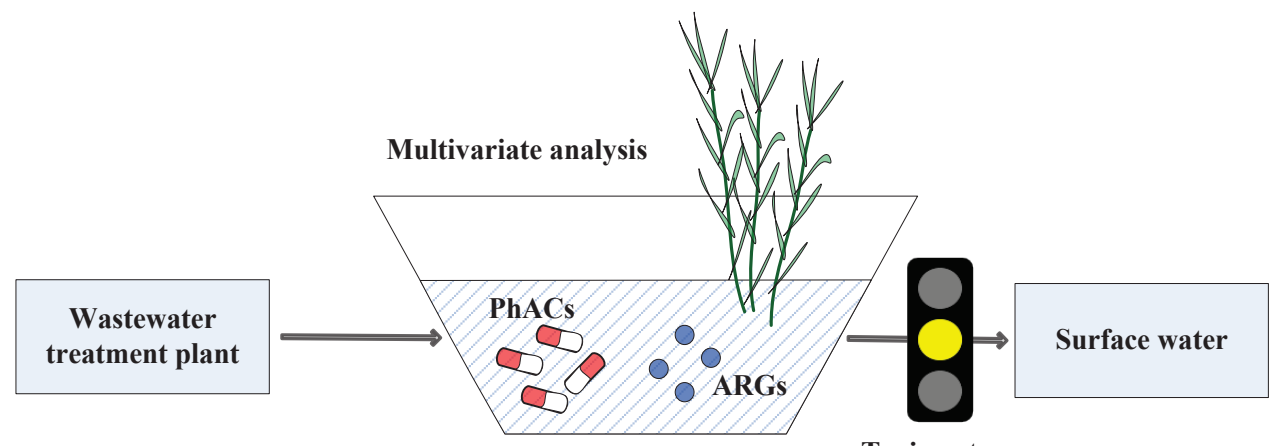

Constructed wetland

Toxic potency 


\section{Chapter 2}

Evaluation of attenuation of pharmaceuticals, toxic potency, and antibiotic resistance genes in constructed wetlands treating wastewater effluents

A modified version of this chapter has been submitted as

He, Y., Nurul, S., Schmitt, H., Sutton, N.B., Murk, T.A.J., Blokland, M. H., Rijnaarts, H.H.M., Langenhoff, A.A.M. Evaluation of attenuation of pharmaceuticals, toxic potency, and antibiotic resistance genes in constructed wetlands treating wastewater effluents. Science of the Total Environment. 


\begin{abstract}
The performance of constructed wetlands (CWs) in the removal of pharmaceutically active compounds (PhACs) is generally evaluated on the basis of chemical analysis. In this work, we used a combination of chemical, toxicological, and molecular analyses to assess the attenuation of PhACs, toxic potency and antibiotic resistance genes (ARGs) in a field study of three different CWs serving as post-treatment of wastewater treatment plant effluents. 17 PhACs were analysed chemically, of which 14 were detected and seven at concentrations greater than $0.1 \mu \mathrm{g} / \mathrm{L}$. Even though some of the PhACs were moderately or highly removed in the CWs investigated, the median removal of PhACs in the vertical subsurface flow $\mathrm{CW}$ was approximately $50 \%$ while the removal in the other two free water surface flow CWs was negligible. Toxic potency of wastewater extracts was assessed in a range of bioassays. Estrogenicity was attenuated in CWs while the neurotoxic potency of wastewater extracts did not decrease after CW treatment. In general, CWs showed a positive removal of a integrase gene and three ARGs tested. Absolute concentrations of some ARGs increased after CW treatment as wells as total bacterial DNA, possibly due to regrowth of resistance-carrying bacteria. Multivariate analysis shows that most PhACs are positively correlated to the observed toxic potency. Additionally, low removal of organics and nutrients seems to parallel with low removal of PhACs. ARGs were found to be correlated to the integrase gene. ARGs also correlated to organics, nutrients and some PhACs but not to the respective antibiotics. In summary, this study demonstrated a comprehensive evaluation of the attenuation of PhACs, toxic potency, and ARGs in field CWs. The actual parameters controlling these attenuation processes need to be further studied to come to a comprehensive understanding of the underlying processes and to an optimal design of CWs as post-treatment facilities for removing micropollutants and ARGs.
\end{abstract}

\title{
Keywords
}

Domestic wastewater treatment; Constructed wetlands; Pharmaceuticals; Bioanalyses; Antibiotic resistance genes; Multivariate analysis 


\subsection{Introduction}

The occurrence of pharmaceutically active compounds (PhACs) in the environment is a growing concern due to their potential threat to the aquatic environment and human health. The term PhACs encompasses a diverse group of compounds, such as antibiotics, hormones, analgesic and anti-inflammatory drugs, $\beta$-blockers, lipids regulator agents, and antiepileptic drugs [235]. Tons of PhACs are consumed on a global scale [324]. For example, although annual consumption in the European Union is generally lower than other areas, nonetheless approximately 15,000 tons of human antibiotics are released yearly into the EU environment [350]. Due to manufacturing processes, improper disposal and metabolic excretion, PhACs are continuously released into the aquatic environment and as a result exhibit a pseudopersistent behaviour [165].

$\mathrm{PhACs}$ are originally made to elicit a biological effect in target organisms [164]. However, with the continuous release of PhACs in the aqueous environment, non-target organisms are and have been exposed over many species generations, which is raising concern towards adverse developmental effects in aquatic ecosystems [118]. Among the diverse pool of $\mathrm{PhACs}$, antibiotics are of particular concern [165] as they may accelerate the development of antibiotic resistance genes (ARGs) in microorganisms. This may compromise the effectiveness of antibiotics in curing diseases in humans and live-stock [194].

Wastewater treatment plants (WWTPs) are the key barrier against the release of PhACs and ARGs to the aquatic environment. However, the conventional treatment processes in WWTPs are not specifically designed for removing PhACs and ARGs, and they are not readily or fully removed $[65,224]$. To further remove PhACs and ARGs from wastewater effluents, posttreatment processes are required. From an economic and environmental perspective, constructed wetland $(\mathrm{CW})$ could be a promising post-treatment technique for removing PhACs and ARGs, as various studies have shown at lab-scale [180, 236, 260, 394], and at field-scale $[64,80,170,262]$.

To date, the performance of CWs related to PhAC removal is in most studies only evaluated on the basis of chemical analysis. However, it is nearly impossible to monitor all PhACs and candidate intermediates in all treatment situations, which is further complicated by complex effects of wastewater matrices on analytics of concentrations in the nano- or microgram per litre level. Furthermore, chemical analysis alone gives limited information to understand the potential effects of PhACs and intermediates on the aquatic environment [349]. Bioanalyses 
employing different test organisms can directly characterize the toxic potency of known and unknown components in a mixed wastewater matrix [391]. To date, such combined chemical and toxicological studies for CWs are limited and no clear correlation has yet been established between those two analyses.

The emergence of ARGs have been confirmed to correlate significantly with antibiotic use [376]. However, correlations between the antibiotic abundance and the ARG levels in the environment are uncertain [376, 383]. Additionally, human-driven changes of natural ecosystems may influence the evolution of ARGs, resulting in antibiotic resistance selection not only through antibiotic selective pressure but also through other chemical pollution such as biocides and detergents $[4,254]$. Therefore, correlations between ARG levels and the abundance of PhACs including antibiotics need to be further investigated.

In the work reported here, we conducted a single sampling campaign and assessed the attenuation performance of CWs on 17 PhACs, toxic potency, and three ARGs based on a combination of chemical, toxicological, and molecular analyses. Three full-scale operating CWs were selected, including two free water surface flow CWs (SF-CWs) and one vertical subsurface flow CW (VSF-CW). Bioanalyses were conducted by employing different varieties of receptors, varying from enzymes to algae. The objectives of this study are to 1) identify the level of PhACs, toxic potency and presence of ARGs in waste effluents and their attenuation in different types of CWs; 2) explore the correlations between levels of PhACs and toxic potency, and between levels of PhACs and ARGs.

\subsection{Materials and methods}

\subsubsection{Chemicals and reagents}

Target PhACs were selected from different categories (Table 2.1), including ketoprofen, diclofenac, ibuprofen, naproxen, erythromycin, lincomycin, sulfamethoxazole, propranolol, metoprolol, clofibric acid, carbamazepine, caffeine, bisphenol A, estrone, 17 $\beta$-estradiol, ethinylestradiol and estriol, which were purchased from Sigma (USA). Properties of the target PhACs are shown in Table S2.1 of the supplementary materials. Other chemicals and reagents used are described in the Text S2.1. 


\subsubsection{Sampling}

Wastewater samples were collected in July 2015 from three CWs acting as post-treatment process of three WWTPs: Land van Cuijk (L), Hapert $(\mathrm{H})$, and Kaatsheuvel $(\mathrm{K})$ in the Netherlands. Detailed background information of related WWTPs and CWs are shown in Table S2.2. CW-L and CW-H are the SF-CWs while CW-K is a VSF-CW.

In order to evaluate the attenuation of PhACs, toxic potency, and ARGs in CWs and the discharge effect of residual PhACs to the following aquatic system, duplicate grab samples were collected at locations indicated in Figure 2.1. The attenuation performance of three CWs were calculated based on the difference between $\mathrm{CW}$ influent and effluent which is represented by L1-L2, H2-H4, and K1-K2, respectively. Water samples were collected in 500 $\mathrm{ml}$ brown glass bottles, transferred to the laboratory the same day, and stored at $4{ }^{\circ} \mathrm{C}$. Sampling bottles were pre-washed with ethanol and deionized water and air dried. For ARG analysis, sampling bottles were further autoclaved at $121^{\circ} \mathrm{C}$ for $20 \mathrm{~min}$ and capped until time of sampling. Pre-treatment of samples (as described in 2.3) was completed within 48 hours before being analysed. Wastewater characteristics were detected: dissolved oxygen (DO), $\mathrm{pH}$, and temperature were analysed using a multi-parameter digital meter (Hach HQ40d, USA); chemical oxygen demand (COD), ammonium $\left(\mathrm{NH}_{4}-\mathrm{N}\right)$, nitrate $\left(\mathrm{NO}_{3}-\mathrm{N}\right)$, and total phosphate (TP) were analysed by using Dr. Lange test kits (Hach Lange GmbH, Germany) on a Hach DR/3900 spectrophotometer. 


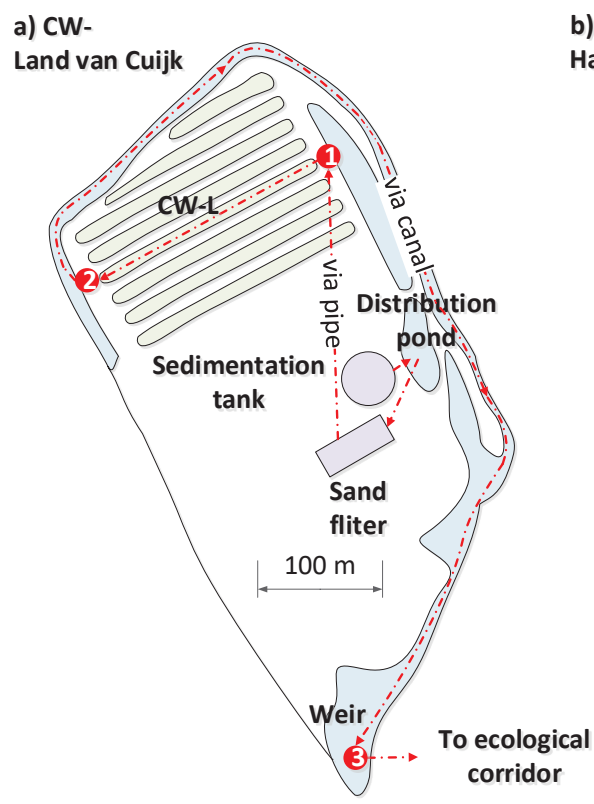

c) CW- b) CW-

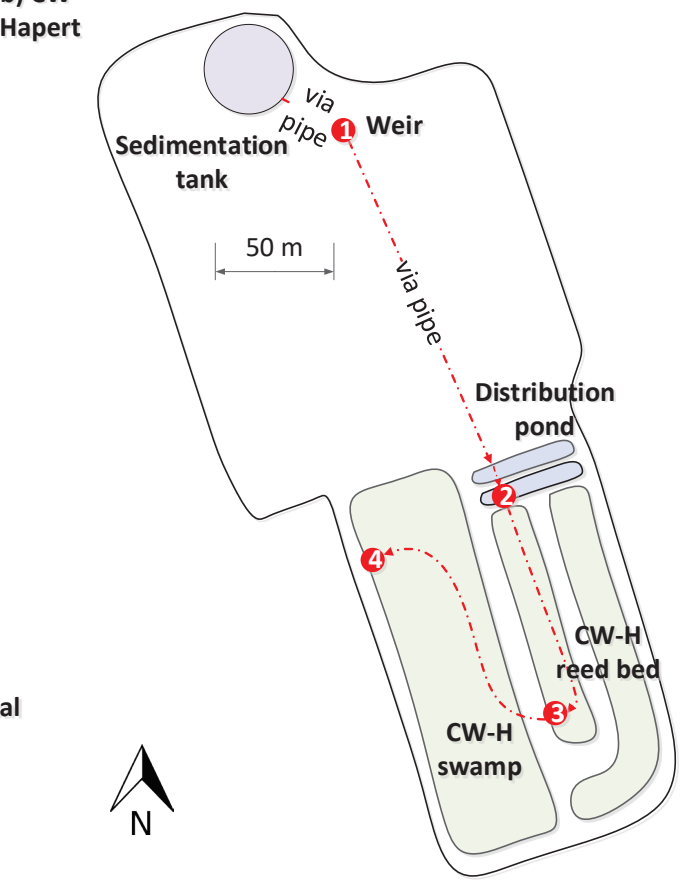

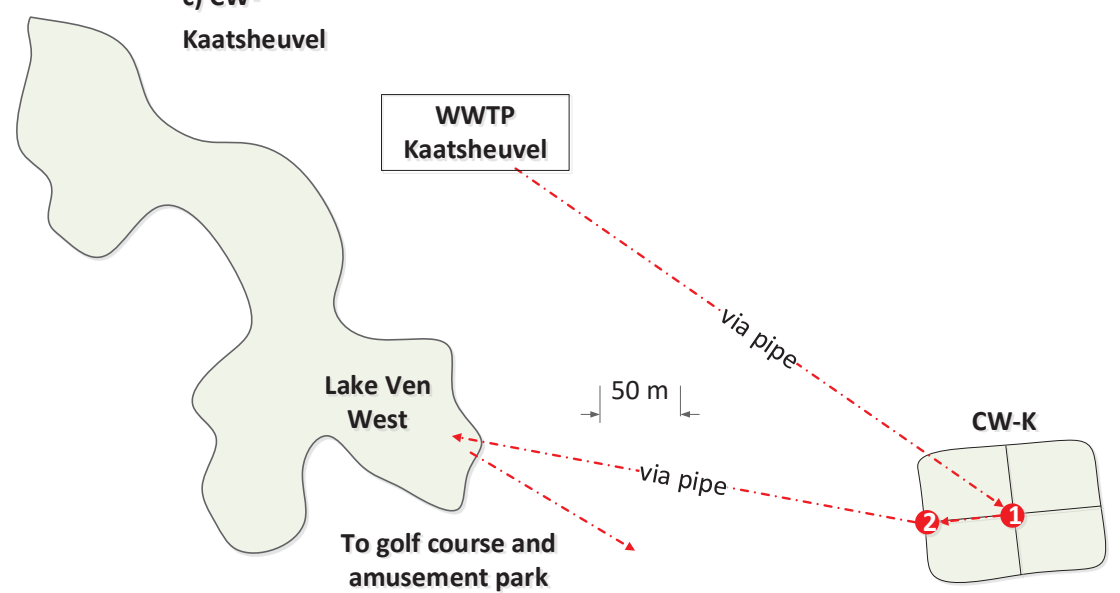

Figure 2.1 Sampling points in three constructed wetlands (CWs) a) Land van Cuijk (L), L1: inlet of CW; L2: outlet of CW; L3: discharge point to an ecological corridor (b) Hapert (H); H1: effluent of sedimentation tank; H2: inlet of CW; H3: after reed bed treatment; H4: discharge point to the surface water; (c) Kaatsheuvel (K). K1: effluent of sand filter/inlet of CW; K2: outlet of CW. Dash lines only indicate the flow direction rather than the real flow paths. The figure was drawn based on the geographical map. 


\subsubsection{Sample pre-treatment}

For chemical and toxicological analyses, samples were pre-treated by filtration $(0.7 \mu \mathrm{m}$ glass filters, GF/F, Whatman, USA) and solid phase extraction (SPE). The pre-treatment procedure is described in detail in Text S2.2 and Figure S2.1. In general, $400 \mathrm{ml}$ of samples was loaded on the SPE cartridges to obtain $12 \mathrm{ml}$ elute, of which $3 \mathrm{ml}$ elute was evaporated to achieve a final $500 \mu \mathrm{l}$ extract with $10 \%$ methanol for chemical analysis while $9 \mathrm{ml}$ elute to $500 \mu \mathrm{l}$ dimethyl sulfoxide (DMSO) for bioanalysis. During SPE, 17 -estradiol-d3 and 10,11dihydrocarbamazepine were added as the internal standards for gas chromatography tandem mass spectrometry (GC-MS/MS) and ultra-high-performance liquid chromatography (UHPLC)-MS/MS analysis, respectively. Recovery of individual PhACs were tested by spiking PhACs in two different matrices, deionized water and wastewater effluent collected from Bennekom WWTP, the Netherlands. Recovery rates are summarized in Table S2.3. As recovery of diclofenac was low from wastewater, concentration of diclofenac was determined by direct injection on UHPLC-MS/MS after being pre-treated by centrifugation at 10,000 rpm for $10 \mathrm{~min}$ (Microlite, Thermo IEC, USA).

For ARG analysis, $500 \mathrm{ml}$ water samples were filtered using $0.2 \mu \mathrm{m}$ membrane filter (Merck Milipore, Ireland) and the filter was placed in centrifuge tubes. Those tubes were stored at $20^{\circ} \mathrm{C}$ before DNA extraction.

\subsubsection{Chemical and bioanalyses}

Hormones were analysed on a GC-MS/MS and quantification of other PhACs was performed by a UHPLC-MS/MS. Detailed analytical methods are shown in Text S2.3, Table S2.4, and Table S2.5.

Bioanalyses to quantify the toxic potency were performed by using 96 -wells plates and detected by a plate reader (Tecan infinite M200 PRO, Switzerland). Different receptors, including yeast, green algae, acetylcholinesterase (AchE) and luminescence bacteria were exposed to the wastewater extracts to determine their acute and chronic toxic potency (Table S2.6). The AchE assay quantifies the potency of the compounds present to inhibit the acetylcholine esterase enzyme, a measure of neurotoxic potency. The REA (RIKILT Estrogen Assay) with the human estrogen receptor $\alpha(h E R \alpha)$, comparable to the yeast estrogen screening assay, was performed to quantify the estrogenicity specifically for the hER $\alpha$. The microtiter Microtox assay provides measure of general toxic and the microtiter algal growth 
inhibition assay (AGIA) is a measure of the phytotoxicity of the hydrophilic compounds present in the wastewater extracts. Methods were validated by reference compounds (Figure S2.2) and the responses expressed relative to that of assay-specific standards. DMSO was used as the blank control. The emission and excitation spectra of wastewater extract were scanned (Figure S2.3) to make sure that the background of wastewater did not overlap with selected measurement wavelengths in Table S2.6. The wastewater extracts were tested in quintuplicate in microtiter AGIA and in triplicate for the other bioanalyses. More information about the bioanalysis protocols are shown in Text S2.4.

For all the conducted bioanalyses, results were reported in inhibition percentage as well as toxic equivalence concentrations (TEQs, equations 2.1-2.3), which is the concentration of a reference compound used to elicit the same response as the unknown and undefined mixture of compounds actually present [247].

$$
\begin{aligned}
& T E Q_{s}=\frac{E C_{50}(\text { reference compound })}{E C_{50}(\text { sample })}=\frac{C_{\text {reference compound }} \times \text { dilution factor }_{\text {reference compoud }}}{\text { enrichment factor }} \\
& \text { Dilution factor } \text { reference compound }=\frac{\text { volume of reference compound added to the bioassay }}{\text { total volume of the bioassay }}
\end{aligned}
$$

Enrichment factor SPE $=\frac{\text { volume of sample loading in } S P E}{\text { volume of extract }}$

$C_{\text {reference compound }}$ is the $50 \%$ effective concentration $\left(\mathrm{EC}_{50}\right)$ of reference compound added to the wells. In principle, any effect level can be used to derive TEQs other than $\mathrm{EC}_{50}$, provided that the concentration-effect curves are reliable [367]. In this study, dilution factor of reference compound was the same with that of wastewater extracts in each bioanalysis. Therefore, the

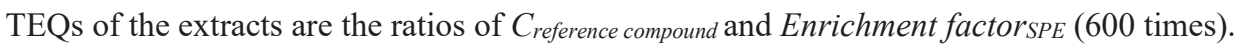

\subsubsection{DNA extraction and ARG quantification}

DNA filters of water samples were conducted using a PowerWater DNA Isolation Kit (MoBio Laboratories, USA), according the manufacturer's protocols. The extracted DNA was stored at $-80^{\circ} \mathrm{C}$ until further analysis.

Quantitative PCR (qPCR) was used to quantify the abundances of 16S-rRNA, the integrase gene Intll and three ARGs, including sull and sul2 (sulfonamide resistance genes), and ermB (macrolide resistance gene). These genes have been recommended for environmental monitoring of antibiotic resistance $[34,141]$ and have been detected in wastewater at high 
prevalence and concentrations [64, 309], making them suitable for analysis of attenuation efficiencies. A synthetic standard known quantity for each gene was used as the standard and DNase free water was used as the blank control. Samples, standards and blanks were run using the same procedure in duplicate. Samples were diluted 50 times to avoid qPCR inhibition by humic acids, biological contaminants or proteins. qPCR assays were conducted using the $\mathrm{iQ}^{\mathrm{TM}}$ SYBR ${ }^{\circledR}$ Green Supermix (Bio-Rad Laboratories, USA) and $\mathrm{iQ}^{\mathrm{TM}}$ Supermix (Bio-Rad Laboratories, USA) for the SYBR Green reactions and TaqMan reactions, respectively. The reaction mixture of $10 \mu \mathrm{l}$ consisted of our sample, master mix, primers (Eurogentec, Belgium), precision blue (Biorad, USA), DNase- and RNase-free water. Details of qPCR conditions and primers are shown in Table S2.7. qPCR assays were conducted on a CFX384 Touch Real-Time PCR Detection System (Bio-Rad Laboratories, Canada). Abundances of genes were normalized to 16S-rRNA values to represent the resistance genes per total bacteria. Detection limit is in the range of $4.81 \times 10^{0}-4.70 \times 10^{7}$ copies $/ \mathrm{ml}$ for $16 \mathrm{~S}$ rRNA, Intll and all ARGs.

\subsubsection{Statistical analysis}

In order to investigate the correlation between removal of PhACs and their physicochemical properties, principal component analysis was conducted by using SIMCA 13.0. In addition, multivariate analysis was conducted using CANOCO 5 software package (Biometrics, the Netherlands) to analyze the correlation 1) among wastewater characteristics, concentration of $\mathrm{PhACs}$ and related toxic potency; and 2) among wastewater characteristics, the abundance of PhACs and ARGs. Response variables in multivariate analysis were centralized and standardized to achieve zero average and unit variance. ARG data was processed by BioRad CFX Manager 3.1 (Biorad, USA).

\subsection{Results and discussion}

\subsubsection{Wastewater characteristics}

The wastewater macro-chemical characteristics $\mathrm{DO}, \mathrm{pH}$, temperature, $\mathrm{COD}, \mathrm{NH}_{4}-\mathrm{N}, \mathrm{NO}_{3}-\mathrm{N}$, and TP, as well as removal efficiencies of these by the CWs are presented in Table S2.8. Discharged wastewater of all three CWs complied with the urban wastewater treatment standards (Council Directive 91/271/EEC). The CW-K removed 2-3 times more nutrients than $\mathrm{CW}-\mathrm{L}$ and $\mathrm{CW}-\mathrm{H}$, respectively (Table S2.8). Levels of $\mathrm{NO}_{3}-\mathrm{N}$ and $\mathrm{TP}$ in the wastewater 
stream further decreased with $66 \%$ and $21 \%$ after passing the canal and ponds (L2 to L3), indicating that open waters could attenuate nutrients as well.

\subsubsection{PhACs}

Of the 17 target PhACs, only the lipid regulator clofibric acid and the estrogenic compounds ethinylestradiol and estriol, were not detected in the WWTP effluent before entering the CWs, and estrone and $17 \beta$-estradiol only in a few samples (Table 2.1). It cannot be excluded that this was because the samples were not deconjugated before analysis, as (synthetic) hormones are excreted as conjugates [218] and as such not detected by the chemical analysis. Ketoprofen only was detected in CW-H. Seven out of 14 detected PhACs discharged to the surface water were at concentrations higher than the guidance value of $0.1 \mu \mathrm{g} / 1$ adopted by toxicologists for drinking water [363]. No regulations exist yet for PhACs discharged from WWTPs to the surface water. Concentrations of ibuprofen and bisphenol A even reached the microgram per liter level. The abundance of ibuprofen was also shown by the study of Verlicchi and Zambello [361], in which ibuprofen was found to be the most abundant PhAC out of $137 \mathrm{PhACs}$ in 136 different CW systems with an average of $32 \mu \mathrm{g} / \mathrm{l}$ and $9.8 \mu \mathrm{g} / \mathrm{l}$ in the influent and effluent of horizontal subsurface flow CWs (HSF-CWs), respectively. Bisphenol A levels were higher in CW-L and CW-H than in CW-K. The latter only receives wastewater from households while the other two CWs also receive wastewater from industries as well (Table S2.2). We assumed that part of the detected bisphenol A in CW-L and CW-H might come from the industries, considering bisphenol $\mathrm{A}$ is an industrial compound manufactured in large quantities as a monomer for producing polycarbonate and epoxy resins [183]. Some $\mathrm{PhACs}$ were detected at higher levels in the subsequent sampling point compared to the upstream point closer to the WWTP effluent discharge point [46, 338], which might be caused by deconjugation by bacteria or because of new input of hormones originating from birds and other animals living in that water. Deconjugation has been observed in WWTPs occasionally for antibiotics, hormones, and psychiatric drugs [145, 155, 365].

Among the detected $14 \mathrm{PhACs}$, erythromycin, sulfamethoxazole, propranolol, and metoprolol could be highly removed ( $>75 \%)$ in the compartments of the CWs; diclofenac, naproxen, and lincomycin were removed moderately (30\%-60\%); while there was almost no removal or even an increase for the other compounds such as bisphenol A (Table S2.9). Fate of PhACs was reported to be correlated with their physicochemical properties [361]. In our study, principal component analysis was conducted to investigate the relationships between removal 
efficiencies of PhACs and combination of their properties including the dissociation constant $\mathrm{pK}_{\mathrm{a}}$, the octanol-water partition coefficient $\log \mathrm{K}_{\mathrm{ow}}$ and $\log \mathrm{D}_{\mathrm{ow}} . \log \mathrm{D}_{\mathrm{ow}}$ is the apparent partitioning coefficient considering the combined effect of $\mathrm{pK}_{\mathrm{a}}$ and $\log \mathrm{K}_{\mathrm{ow}}$. The results showed no correlation between PhACs removal and those properties (Figure S2.4). The noncorrelation with $\mathrm{pK}_{\mathrm{a}}$ and $\log \mathrm{K}_{\mathrm{ow}}$ is also in line with previous research, which proved that PhACs behavior in the biological treatment processes cannot be determined by a certain chemical parameter alone [46, 287, 394]. The absence of correlation with a single or combined physical parameters indicates that more removal mechanism (e.g. phytoremediation) might be involved other than biodegradation and adsorption in our case. 
Evaluation of field constructed wetlands

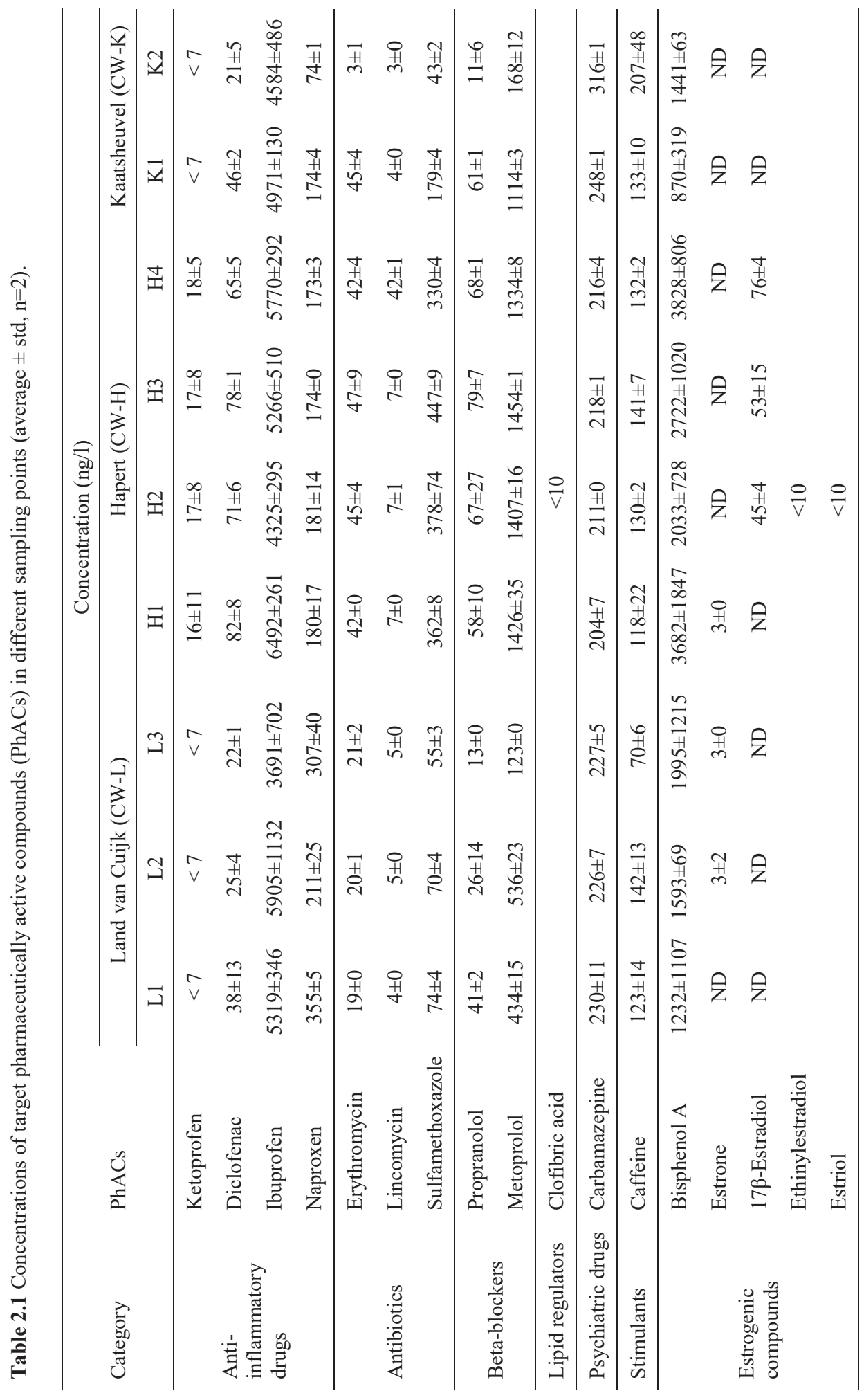




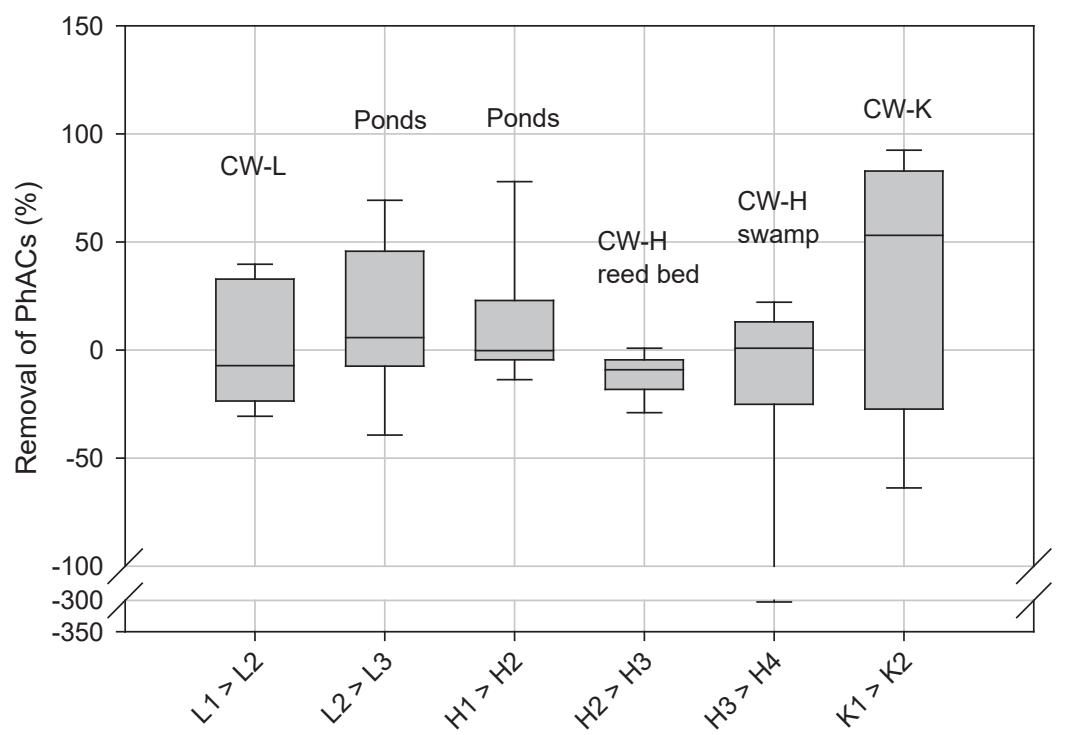

Figure 2.2 Removal efficiencies of 14 detected pharmaceutically active compounds (PhACs) in constructed wetlands (CWs) and open waters. The box plot shows the values in maximum, third quartile, median, first quartile, and minimum.

Removal efficiencies of 14 detected PhACs were evaluated in three CWs and open waters. Strikingly, the median removal of PhACs in CW-K was approximately $50 \%$ while that in $\mathrm{CW}-\mathrm{L}$ and $\mathrm{CW}-\mathrm{H}$ (both reed bed and swamp compartment) was negligible (Figure 2.2). The removal efficiencies we observed are relatively low compared with previous studies where removal of most investigated PhACs in CWs were higher than 50\% [170,262]. The better $\mathrm{PhAC}$ removal performance of CW-K might be caused by its vertical configuration and operational parameters such as hydraulic loading rate (HLR). On the one hand, CW-K is a VSF-CW while the other two CWs are SF-CWs. Compared with SF-CWs, VSF-CWs usually achieve a better oxygenation and possess a superior rhizosphere effect in rhizodegradation as well as adsorption [259, 400]. In fact, Matamoros et al. [262] reported that more than $50 \%$ of the studied PhACs were better and more consistently removed in the VSF-CWs as compared with other technologies such as compact bio-filters and biological sand filters. On the other hand, a lower HLR in CWs was reported to result in a higher removal of PhACs due to longer contact and interaction among nutrients, substrate and roots [16, 394]. In our study, CW-K with a lower HLR (Table 2.1) indeed showed higher removal for PhACs. 
PhACs could be attenuated in open waters (L2 to L3, H1 to H2 in Figure 2.2), in which PhACs are directly exposed to the sunlight. In fact, PhAC removal has been verified in ponds, either as polishing ponds followed by CWs or as post-treatment units in WWTPs, in which photodegradation might play an important role [168, 266, 311]. Therefore, CWs are suggested to include shallow open water compartments to enhance photodegradation of PhACs. But still, CWs with plants are useful as they are rich in biomass and thus are less affected by seasonal changes for removing biodegradable PhACs compared with ponds [266].

\subsubsection{Toxic potency}

In the present study, toxic potency of wastewater extracts was assessed by five bioanalyses based on different receptors. Results are expressed as inhibition or response to receptors and relative to that of the reference standard (toxic equivalence concentrations, TEQs). In the microtiter AGIA and Microtox assays no toxicity was observed (Figure S2.5). Interestingly, the vitality of the algae and bacteria was even enhanced when wastewater extracts were added, possibly because the extracts contained nutrients. It was confirmed that this was not due to background color or fluorescence from the extracts. The same enhancement phenomenon was also found in previous studies [156, 241].

In the YTA, toxic potency of wastewater extracts were notably attenuated in CW-H but not in the other two CWs (Figure 2.3a). The retained toxic potency is of environmental concern reflected from the TEQtributyltin observed (0.85-3.33 $\mu \mathrm{g} / \mathrm{l})$. It was reported that half of 7 day-old larvae of mussels (Mytilus edulis) died upon exposure to $0.1 \mu \mathrm{g} / 1$ tributyltin for 15 days and all the larvae were dead under $10 \mu \mathrm{g} / 1$ tributyltin exposure for 5 days [31]. The AchE inhibiting potency was comparable in all wastewater extracts, indicating that the operational compartments had no capacity to attenuate the toxic potency (Figure 2.3b). The TEQ dichlorvos was in the range of 6.4-11.8 $\mu \mathrm{g} / 1$ for surface water discharging points. This level of dichlorvos could already inhibit the activity of AchE (Figure S2.2) resulting in in an over-stimulation of the postsynaptic elements, i.e. neuron, muscle and gland in humans and animals. 

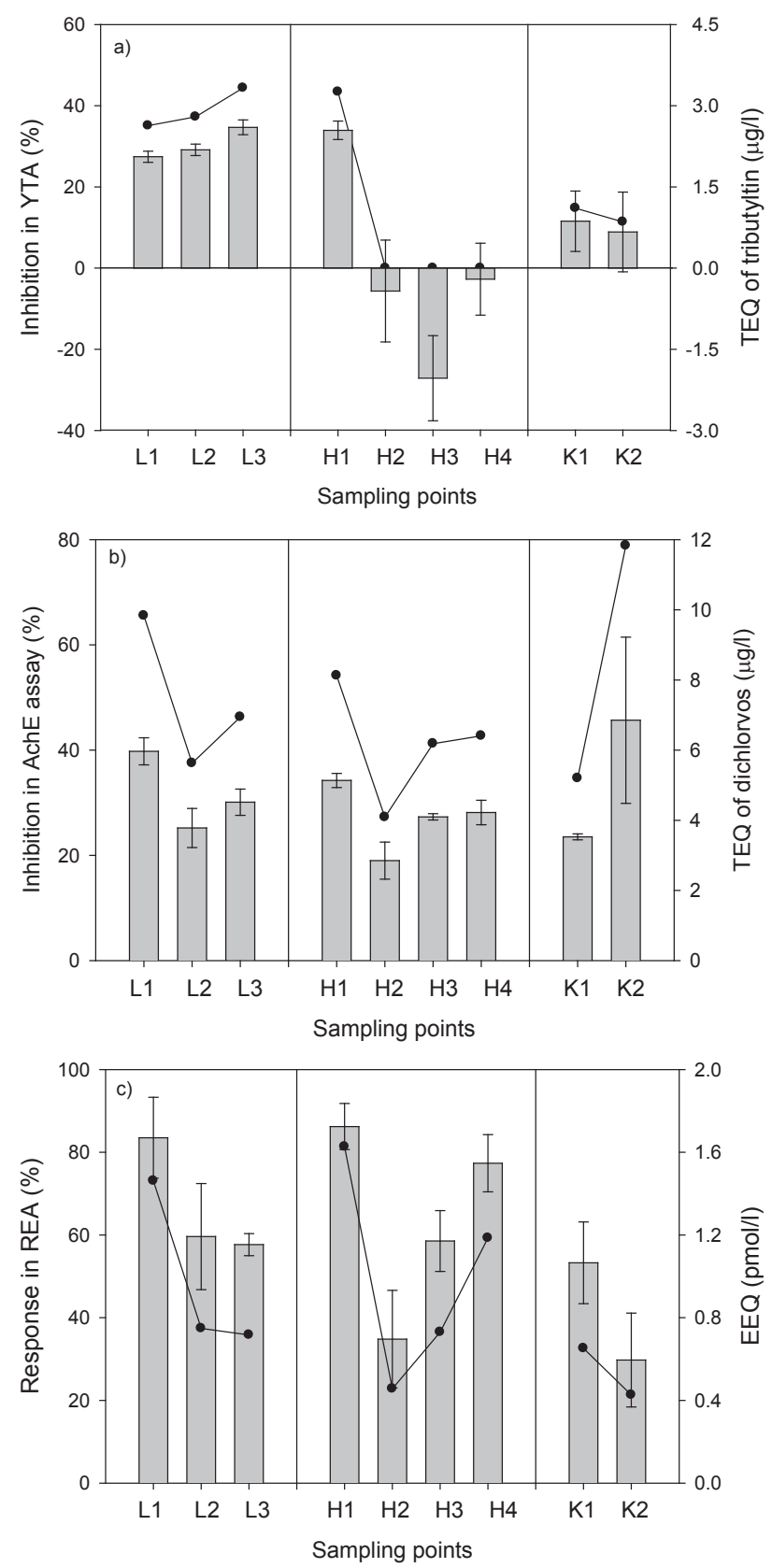

Figure 2.3 Toxic potency and toxic equivalence concentrations (TEQs) of wastewater extracts in various compartment positions in the $\mathrm{CW}$ for three different bioanalyses: a) yeast toxicity assay (YTA); b) acetylcholinesterase (AChE) assay; c) RIKILT Estrogen Assay (REA). The left axis corresponds to data in bars; the right axis corresponds to data in dots. Toxicity data are mean value \pm standard error $(n=6)$. 
The estrogenic potency of the sample extracts, as analysed in the REA, decreased overall in sequential operational compartments, from point L1 to L3, H1 to H2, and K1 to K2 (Figure 2.3b). Only in $\mathrm{H} 3$ and $\mathrm{H} 4$ the potency increased, which happen to be the few locations in which $17 \beta$-estradiol was found with the chemical analysis. The observed $17 \beta$-estradiol may originate from additional sources such as hormones from the inhabitant birds, as was previously reported for $\mathrm{CW}-\mathrm{H}$ [128]. In our study, the level of estradiol equivalents (EEQ) was 0.4-1.6 pmol/1 (Figure 2.3b), which is of the same magnitude as the EEQ detected in Dutch wastewater effluent in previous studies: 0.9-2.5 pmol/1 [128] and 0-2.1 pmol/1 [278]. Even though the estrogenicity decreased in operational compartments before discharging to surface water bodies, still the observed estrogeniticity level is of concern. A previous study showed that $1.3 \mathrm{pmol} / 1$ EEQ could affect immature male rainbow trout to produce estrogen biomarker vitellogenin after 28 weeks of dosing [326].

In summary, wastewater extracts showed in general toxicity in YTA and specific toxicity in REA and AChE assays. The TEQ levels reflected from different assays are of environmental concern. Especially no attenuation of the toxic potency in the AchE assay was observed after $\mathrm{CW}$ treatment.

\subsubsection{Antibiotic resistant genes}

In the present study, the integrase gene (intll) and three ARGs (sul1, sul2, and ermB) were investigated in the wastewater. All ARGs were detected in the wastewater samples except that ermB was under detection limit at K2 (Figure 2.4a). Overall, the class 1 integron gene intl had the highest concentrations. Among ARGs, the abundance of sull was highest followed by sul2 and ermB, in terms of both absolute concentrations and concentrations relative to the total bacterial community. The detected ARG concentrations varied from 4.9 copies $/ \mathrm{mL}$ (ermB, L2) to $1.7 \times 10^{5}$ copies $/ \mathrm{mL}$ (sul1, H4) in wastewater samples (Figure 2.4a). The findings in this study are in line with previous studies in which sull and sul2 genes were the most abundant ARGs in CWs [64, 65], rivers [61, 214, 295], and marine environments [340]. 

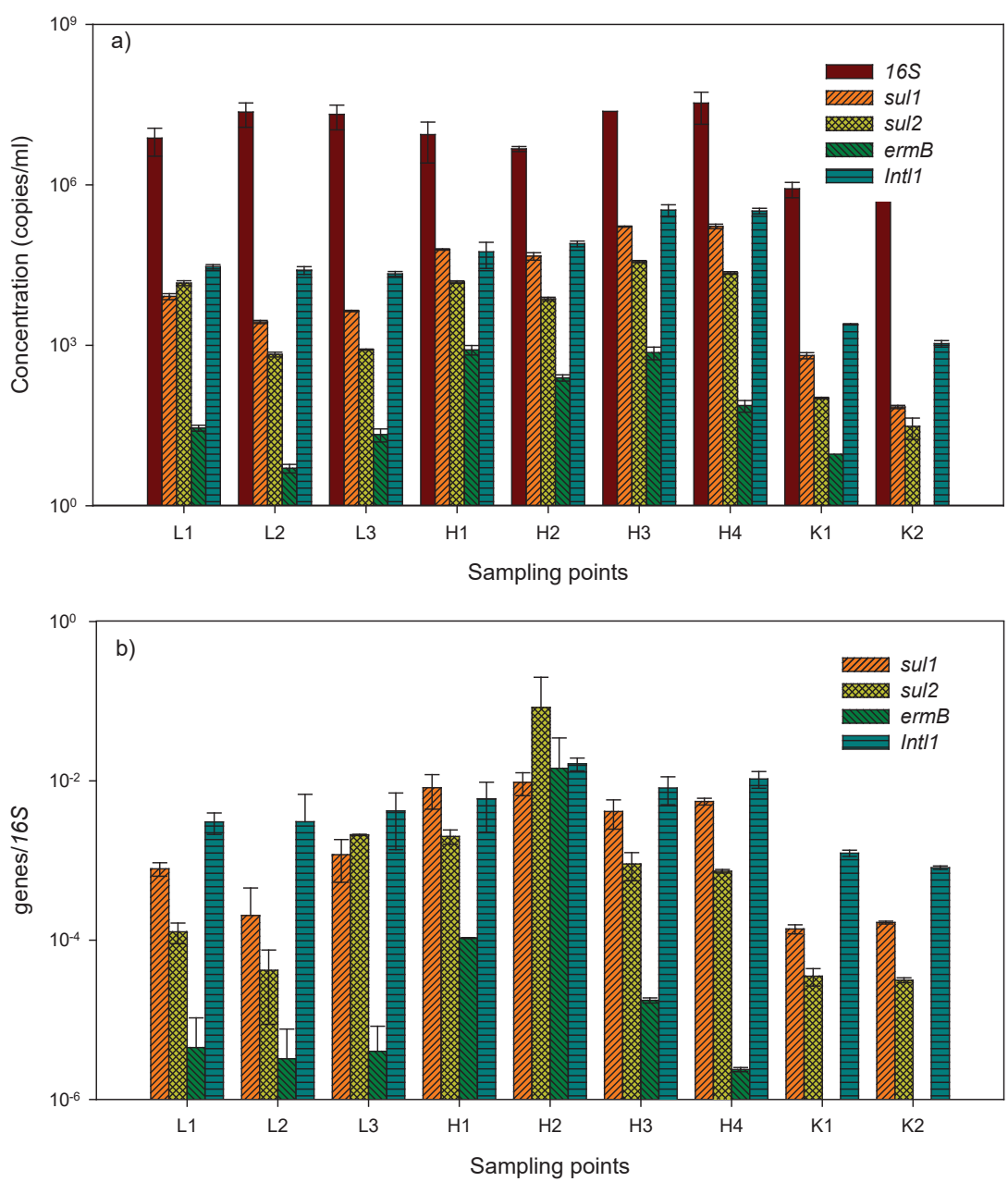

Figure 2.4 The a) absolute concentrations and b) normalized concentrations of genes in three constructed wetlands CW-L, CW-H and CW-K. Results are mean value \pm standard deviation $(n=2)$.

$\mathrm{CW}-\mathrm{L}$ and $\mathrm{CW}-\mathrm{K}$ showed positive removal of the absolute concentrations of all ARGs in the range of $14 \%$ (Intll) to $95 \%$ (sul2), and $57 \%$ (Intll) to $100 \%$ (ermB) (Table S2.10). Meanwhile, CW-H showed negative removal (i.e. increase) of all ARGs except for ermB (70\%). Notably, the total bacteria increased in all the investigated CWs (Table S2.10). Relative to the total bacterial, most resistance genes remained stable or showed a decrease after CW treatment (L1-L2, H2-H4, K1-K2, Figure 2.4b). Some of the previous researches concluded that CWs are able to reduce the concentration of ARGs [13, 65, 117], while some also observed a significant increase in antibiotic resistance after $\mathrm{CW}$ treatment, either in 
absolute concentrations [282] or relative concentrations [177, 236]. Suitable conditions can promote regrowth of microorganisms after the treatment [404], which might lead to the observed absolute increases of ARGs. In addition, selective pressures might be present that also promote regrowth of resistance-carrying bacteria, including antibiotic selective pressure which might be present even at low concentrations [149, 343], interaction mediated by antibiotics or non-antibiotic metabolites [36], or heavy metal selective pressure [19].

\subsubsection{Multivariate analysis of PhACs, toxic potency, and ARGs data}

Multivariate analysis was conducted to explore chemical, toxicological and molecular outcomes, and their correlation was investigated through projections onto the ordinations obtained. The detected toxic potency is positively correlated to wastewater characteristics and PhACs (Figure 2.5a). Various researchers have positively correlated organics (COD) and nutrients to the toxic potency of wastewater [30, 245, 391]. In contrast, PhACs have rarely been correlated with toxic potency in real wastewater matrixes. In our study, toxic potency described in REA and YTA seemed to be positively correlated with wastewater characteristics and PhACs. Especially REA positively correlated to organics, nutrients $\left(\mathrm{NH}_{4}-\mathrm{N}, \mathrm{NO}_{3}-\mathrm{N}, \mathrm{TP}\right)$ as well as most of the PhACs. In comparison, AchE correlated less with environmental variables, indicating that the neurotoxic potency of wastewater extracts might be related to other pollutants than the PhACs we tested.

ARG levels are not correlated to the abundance of related antibiotics but rather to organics, nutrients, and some PhACs (Figure 2.5b). Higher concentrations of SMZ and ETM did not correlate with higher concentrations of $\operatorname{sul1/2}$ and $\operatorname{ermB}$, respectively. This lack of correlation may result from three reasons: 1) Wastewater already contains high amounts of resistance genes, which are not necessary related to the actual wastewater antibiotic content. 2) Abundance of resistance gene in the CWs result from survival (or even growth) of wastewater bacteria carrying these genes, or selection of resistant bacteria in situ. These processes are in turn possibly partly, but not exclusively mediated by PhACs or other selective pressures. 3) Mobile genetic element such as plasmids, integrases, and transposases are able to assist the spread of ARGs without antibiotics being present [412]. Thus, antibiotics and resistance genes do not necessarily have to be correlated, as also shown in previous research [7, 296, 376]. However, a positive correlation was found between concentrations of ARGs and concentrations of organics, nutrients and some PhACs. This might indicate that organics and 
nutrients stimulate growth of resistant bacteria in CWs, and that processes removing these pollutants also reduce resistance genes to a similar extent.

Sul1, sul2 and ermB show strong correlations with intll (Figure 2.5b), indicating that removal or regrowth of bacteria harboring these genes in general co-occurs. The intll gene has been found to be correlated with the dissemination of both types of sul genes in the environment [62]. The sull gene is normally found in class 1 integrons intl1 [330], whereas sul2 is usually located on small non-conjugative plasmids [111] or large transmissible, multi-resistance plasmids [166]. In a study of Antunes et al. [11], they observed intl1 was presence in almost $98 \%$ of sull isolates. Shehabi et al. [327] also found that $62 \%$ of sull/sul2 was positively associated with intll. With this correlation, Muziasari et al. [279] suggested that intll may play a role in the prevalence of sull through horizontal gene transfer.

As indicated in Figure 2.5a and 5b, the observed positive correlation between concentrations of organics and nutrients and concentrations of PhACs shows that conditions that remove organics and nutrients most likely remove PhACs as well. Similar results were found in the study of Matamoros et al. [259], in which the authors positively linked the high removal of most targeted PhACs with the high removal of BOD, total suspended solid (TSS), and $\mathrm{NH}_{4}-\mathrm{N}$ in a VSF-CW and sand filter systems. Therefore, the low removal of organics and nutrients might explain the low attenuation of PhACs in this study.

Finally, it should be noted that the single sampling campaign executed in this study may limit the evaluation of $\mathrm{CW}$ attenuation performance. It cannot be excluded that possible dilution or concentration of compounds occurred depending on the amount of rain water received. However, the multivariate analysis implemented in this study provides more insight into the presence and removal of PhACs as well as their associated environmental hazards (i.e. toxic potency and ARGs). These results overall show a snapshot of limited and variable attenuation of PhACs, toxic potency and ARGs in the three CWs. The findings might indicate many removal processes in the CWs are sub-optimal and more knowledge generation on the attenuation mechanisms under varying $\mathrm{CWs}$ operational conditions is essential. More repeated measurement would be conducted in the future to confirm this indication. 

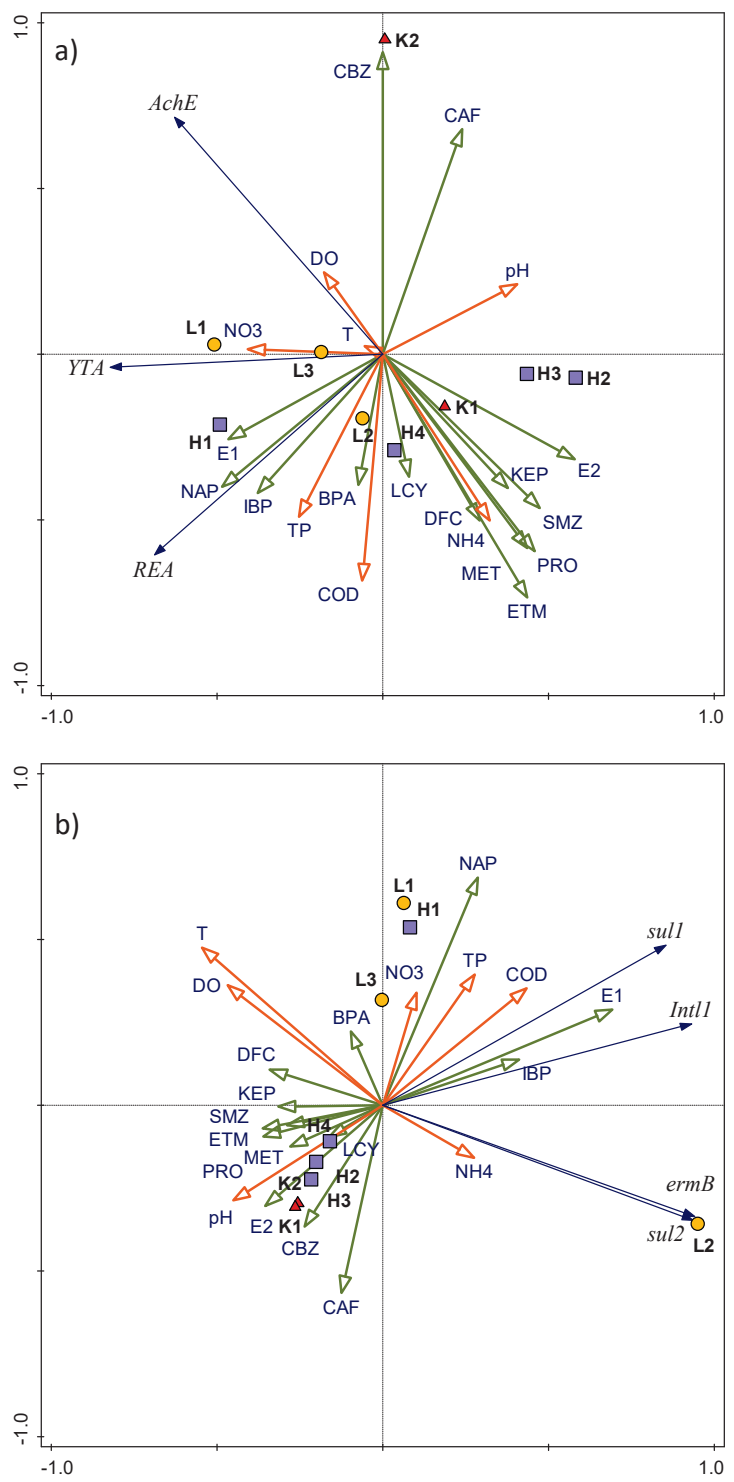

Figure 2.5 Multivariate analysis of the correlation a) among toxic potency, wastewater characteristics and PhACs; b) among ARGs (normalized), wastewater characteristics and PhACs. Sampling points are indicated in circles for $\mathrm{CW}-\mathrm{L}$, rectangles for $\mathrm{CW}-\mathrm{H}$, and triangles for $\mathrm{CW}-\mathrm{K}$, respectively. Environmental variables are shown in arrows. The length of arrows indicates variance that can be explained by the environmental variables while the direction of arrows indicates the magnitude of variables. The intersection angle between arrows represents their correlation, in which a more acute intersection angle means a stronger correlation. The eigenvalues of the first and second canonical axis are 0.51 and 0.29 in a), 0.84 and 0.13 in b). 


\subsection{Conclusions}

In this study, performance of CWs to attenuate PhACs, toxic potency, and ARGs has been assessed. Furthermore, correlations between toxic potency, PhACs, ARGs, and water characteristics were explored. The main findings are: 1) Several PhACs discharged to the surface water were at concentrations higher than $0.1 \mu \mathrm{g} / \mathrm{l}$, especially for bisphenol A and ibuprofen. Even though some of the PhACs were moderately or highly removed, the median removal of PhACs in CWs was approximate $50 \%$ in $\mathrm{CW}-\mathrm{K}$ and negligible in other two CWs. No correlation was found between the removal efficiencies of PhACs and their physicochemical properties. 2) Wastewater extracts showed general toxicity in YTA and specific toxicity in REA and AChE assays. The TEQ levels reflected from bioanalyses are of environmental concern and the toxic potency did not show attenuation in the AchE assay. 3) Positive ARG removal was observed in CW-L and CW-K in terms of both absolute and relative concentrations. The increased absolute concentrations of sul1, sul2, and Intl1 in CW$\mathrm{H}$ as wells as the increase of total bacteria in all CWs may link to regrowth of microorganisms mediated by suitable growth conditions and/or selective pressures. 4) Most PhACs were positively correlated to the toxic potency, indicating a potential hazard of these compounds to the environment. Concentrations of organics, nutrients, and some PhACs were positively correlated to ARG concentrations while no concrete pattern of ARGs can be predicted from the concentration of the antibiotics analysed. Intll showed positive correlation with all three ARGs. The low removal of organics and nutrients might explain the low attenuation of $\mathrm{PhACs}$ due to their positive correlations.

Considering the insufficient removal of PhACs, toxic potency and ARGs in CWs, enhancement of $\mathrm{CW}$ performance is desirable, where optimal construction (e.g. vertical configuration, constructed with open waters) and operational parameters (e.g. HLR) can be considered. Multivariate analysis in this study offers a great potential to comprehensively evaluate the performance of CWs by associating chemical, toxicological, and molecular analyses. 


\section{Acknowledgement}

Authors thank Hennie van Rossum for the technical help with GC-MS/MS analysis, and thank Mark Elders, Kasper Kusters, Peter van Dijk, Mario Dieltjes, and Joost van den Bulk for their support of sampling and supplying wetland information. The support provided by China Scholarship Council (CSC) for the research of Yujie He at Wageningen University is kindly acknowledged. 


\section{Supplementary materials}

Text S2.1 Chemicals and reagents

Methanol, formic acid, ammonium formate and water (Actu-All Chemicals, the Netherlands) used for ultra-high-performance liquid chromatography tandem mass spectrometry (UHPLCMS/MS) analysis was of UPLC grade. The derivatization reagent MSTFA++ was a mixture of N-methyl-N-trimethylsilyl-trifluoroacetamide from Alltech (Anaconda, MT, US), ammonium iodide from Fluka (Zwijndrecht, The Netherlands), and dithioerythreitol from AnalaR $(1000: 2: 4, \mathrm{v} / \mathrm{w} / \mathrm{w})$. All other chemicals and reagents used were of analytical grade or higher. Deionized water from a Milli-Q system (Millipore, USA) was used to prepare solutions. DMSO used in bioassays was purchased from Sigma ( $\geq 99.5 \%$, USA).

Text S2.2 Pre-treatment of water samples for chemical and toxicological analyses

For chemical and bioanalysis, samples were filtered through $0.7 \mu \mathrm{m}$ glass filters $(\mathrm{GF} / \mathrm{F}$, Whatman, USA) prior to solid phase extraction (SPE). Oasis HLB cartridges (6 cc/60 mg, Waters, USA) were used for the SPE. Firstly, $\mathrm{pH}$ of water samples was adjusted by adding 4 $\mathrm{ml}$ buffer ( $\mathrm{pH}=10$, Merck, Germany). The cartridge was pre-conditioned with $5 \mathrm{ml}$ methanol and then equilibrated with $5 \mathrm{ml}$ deionized water. Next, $400 \mathrm{ml}$ of samples passed through SPE cartridges with a flow of 5-10 $\mathrm{ml} / \mathrm{min}$ controlled by a vacuum pump (Buchi V-700, Switzerland). After loading samples, cartridges were washed with $12 \mathrm{ml}$ deionized water and eluted with $12 \mathrm{ml}$ of $25 \% \mathrm{NH}_{4} \mathrm{OH}$ : methanol (8/92, v/v). The eluent of each $400 \mathrm{ml}$ sample was divided into $3 \mathrm{ml}$ and $9 \mathrm{ml}$ for chemical and bioanalysis, respectively (Figure S2.1). The $3 \mathrm{ml}$ eluent was evaporated to $200 \mu \mathrm{l}$ at $35^{\circ} \mathrm{C}$ under a gentle stream of nitrogen (VLM evaporator, Germany). Then, $250 \mu \mathrm{l}$ Milli-Q water was added and the remaining methanol $(200 \mu 1)$ was evaporated. As a final step, the extract was adjusted with water:methanol (80:20, $\mathrm{v} / \mathrm{v}$ ) by weight to achieve a final $500 \mu \mathrm{l}$ extract with $10 \%$ methanol. The $9 \mathrm{ml}$ eluent was evaporated to dryness and replaced with $500 \mu \mathrm{DMSO}$ by weight. 
Text S2.3 Chemical analysis of pharmaceutially active compounds (PhACs)

Hormones were analysed on a gas chromatography (GC)-MS/MS. Samples were derivatized prior to chemical analysis. The $10 \%$ methanol extract was evaporated at $60{ }^{\circ} \mathrm{C}$ to dryness under a gentle nitrogen flow. The dry residue was derivatized by adding $25 \mu \mathrm{MSTFA}^{++}$ followed by incubation at $60{ }^{\circ} \mathrm{C}$ for $1 \mathrm{~h}$. The derivatized extract was evaporated at $60{ }^{\circ} \mathrm{C}$ to dryness and reconstituted with $25 \mu \mathrm{l}$ of iso-octane. Afterwards, the extract was sonicated for 2 min and mixed by vortexing. GC-MS/MS analysis was performed on a Varian 1200 triple quadrupole MS system (Varian, USA) comprised of a Varian CP 3800 GC and a Varian CP 8400 auto-sampler. The GC was equipped with a J\&M GC column $(30 \mathrm{~m} \times 0.25 \mathrm{~mm}, 0.25 \mu \mathrm{m}$, Agilent, USA). $2 \mu \mathrm{l}$ of samples were injected in splitless mode to the GC column by a pulsed pressure of $30 \mathrm{psi}$ for $1.2 \mathrm{~min}$. The injector was kept at $250{ }^{\circ} \mathrm{C}$ for $1 \mathrm{~min}$. The oven temperature program was as follows: $110^{\circ} \mathrm{C}$ (held for $1 \mathrm{~min}$ ), ramped at $20^{\circ} \mathrm{C} / \mathrm{min}$ to $250{ }^{\circ} \mathrm{C}$ (held for $2 \mathrm{~min}$ ), then ramped at $5{ }^{\circ} \mathrm{C} / \mathrm{min}$ to $280{ }^{\circ} \mathrm{C}$, and finally ramped at $25{ }^{\circ} \mathrm{C} / \mathrm{min}$ to $330{ }^{\circ} \mathrm{C}$ (held for $2 \mathrm{~min}$ ). Helium was used as the carrier gas and the flow was kept at $1 \mathrm{ml} / \mathrm{min}$. Selected ion monitoring (SIM) mode was conducted to analyse E3 while the other hormones were measured in multiple reaction monitoring (MRM) mode. Detailed transitions and collision energies are listed in Table S2.4.

Quantification of other PhACs was performed by using a Waters UHPLC Acquity system coupled to a Waters Xevo TQ MS. $10 \mu$ l extract was injected on a Atlantis HILIC Silica T3 column $(3.0 \times 100 \mathrm{~mm}, 3 \mu \mathrm{m})$ (Waters, USA) and was separated using gradient elution with a stable flow of $0.4 \mathrm{ml} / \mathrm{min}$. The solvent used were A: water/ammonium formate/formic acid (1000/2/0.16) and B: methanol/ammonium formate/formic acid (1000/2/0.16). The gradient was set as: $0-0.5 \mathrm{~min} 10 \% \mathrm{~B}$; 0.5-6 min linear increased to $70 \% \mathrm{~B}$; 6-7 linear increased to $100 \%$ $\mathrm{B}$ and hold $1 \mathrm{~min} ; 8-8.1$ decreased to $10 \% \mathrm{~B}$ and hold until $10 \mathrm{~min}$. Column temperature was maintained at $60{ }^{\circ} \mathrm{C}$. Waters Xevo TQ was operated in MRM mode using electrospray ionization. The instrument conditions for positive mode were: capillary voltage $2.2 \mathrm{kV}$, cone voltage $40 \mathrm{~V}$, desolvation gas flow $800 \mathrm{~L} / \mathrm{h}$ at $600{ }^{\circ} \mathrm{C}$, con gas flow $150 \mathrm{~L} / \mathrm{h}$, collision gas flow $0.18 \mathrm{ml} / \mathrm{min}$. While in negative mode, capillary voltage was $1.5 \mathrm{kV}$ and other parameters were the same with positive mode. Product ions were chosen for confirmation, in which the most intensive product ion was selected for the quantification. MS parameters including optimized collision energy were summarized in Table S2.5. Peak identification and quantification was performed using MassLynx software version 4.2. 
Text S2.4 Procedure of five bioanalyses. Percentages of DMSO in wells were $0.5 \%, 1.0 \%$, $1.7 \%, 0.5 \%$, and $0.9 \%$ in the following five bioanalyses respectively.

YTA. The Yeast Toxicity Assay was adjusted based on Fai and Grant [113]. Toxic potency of wastewater samples was quantified relative to the lethality of yeast cells induced by tributyltin. S.cerevisiae, a regular baking and brewing yeast, was purchased from the local supermarket. Briefly, 0.15-0.18 g yeast was activated by adding into $100 \mathrm{ml}$ of the mixture of phosphatebuffered saline (PBS) and glucose and incubating for $1 \mathrm{~h}$ at $30^{\circ} \mathrm{C}$. The mixture was prepared by mixing 1 pill of PBS and $2 \mathrm{~g}$ glucose in $100 \mathrm{ml}$ deionized water. Next, $5 \mathrm{ml}$ of tributyltin or samples were diluted with $495 \mathrm{ml}$ of the PBS/glucose mixture. $100 \mathrm{ml}$ of the diluted tributyltin or samples were added to the plate in triplicate. Afterwards, $70 \mathrm{ml}$ yeast suspension and $30 \mathrm{ml}$ redox dye resazurin were added to each well as substrate. Fluorescence of resorufin was analysed after $1 \mathrm{~h}$ of cultivation at $30^{\circ} \mathrm{C}$.

REA. RIKILT Estrogen Assay with the human estrogen receptor $\alpha(\mathrm{hER} \alpha)$ was performed based on the study of Bovee et al. [42]. Yeast used was genetically modified to express human estrogen receptor-beta by RIKILT, Wageningen University and Research, the Netherlands.

AchE assay. Acetylcholinesterase (AchE) assay was performed based on previous studies $[152,154]$. AchE is a key enzyme for stopping signal transmission in humans and animals after excitation. The neurotoxic potency of xenobiotics is quantified based on their ability to block esterases, thus inhibiting the hydrolysis of the neurotransmitter acetylcholine by AChE $[152,154]$. AchE was extracted from honey bees heads collected from a clean area in Renkum, The Netherlands and prepared according to Hamers et al. [152].

Microtiter Microtox Assay and microtiter AGIA. Luminescence bacteria Vibrio fischeri was purchased from Microlan (the Netherlands). Green algae Pseudokirchneriella subcapitata was obtained from Department of Aquatic Ecology and Water Quality Management, Wageningen University. The microtiter Microtox assay was based on the method developed by Hamers et al. [153] with some modifications as described in $\mathrm{He}$ et al. [156]. The microtitier algal growth inhibition assay (AGIA) was performed as described previously [39, 189]. 
Load $400 \mathrm{ml}$ filtrated sample, duplicate

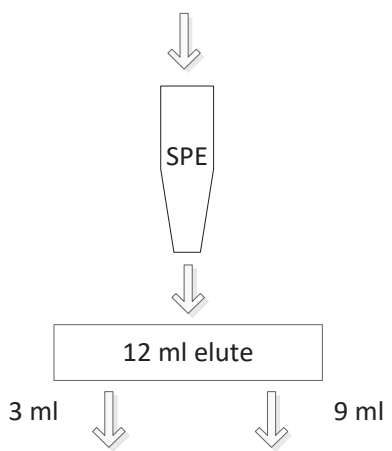

$500 \mu$ lextract in $10 \% \mathrm{MeOH}$,

$500 \mu \mathrm{l}$ extract in DMSO,

200 times concentrated

600 times concentrated

LC-MS/MS analysis for chemical concentration

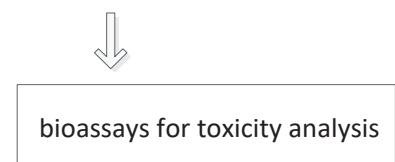

Figure S2.1 Pre-treatment of wastewater samples for chemical and bioanalyses. 

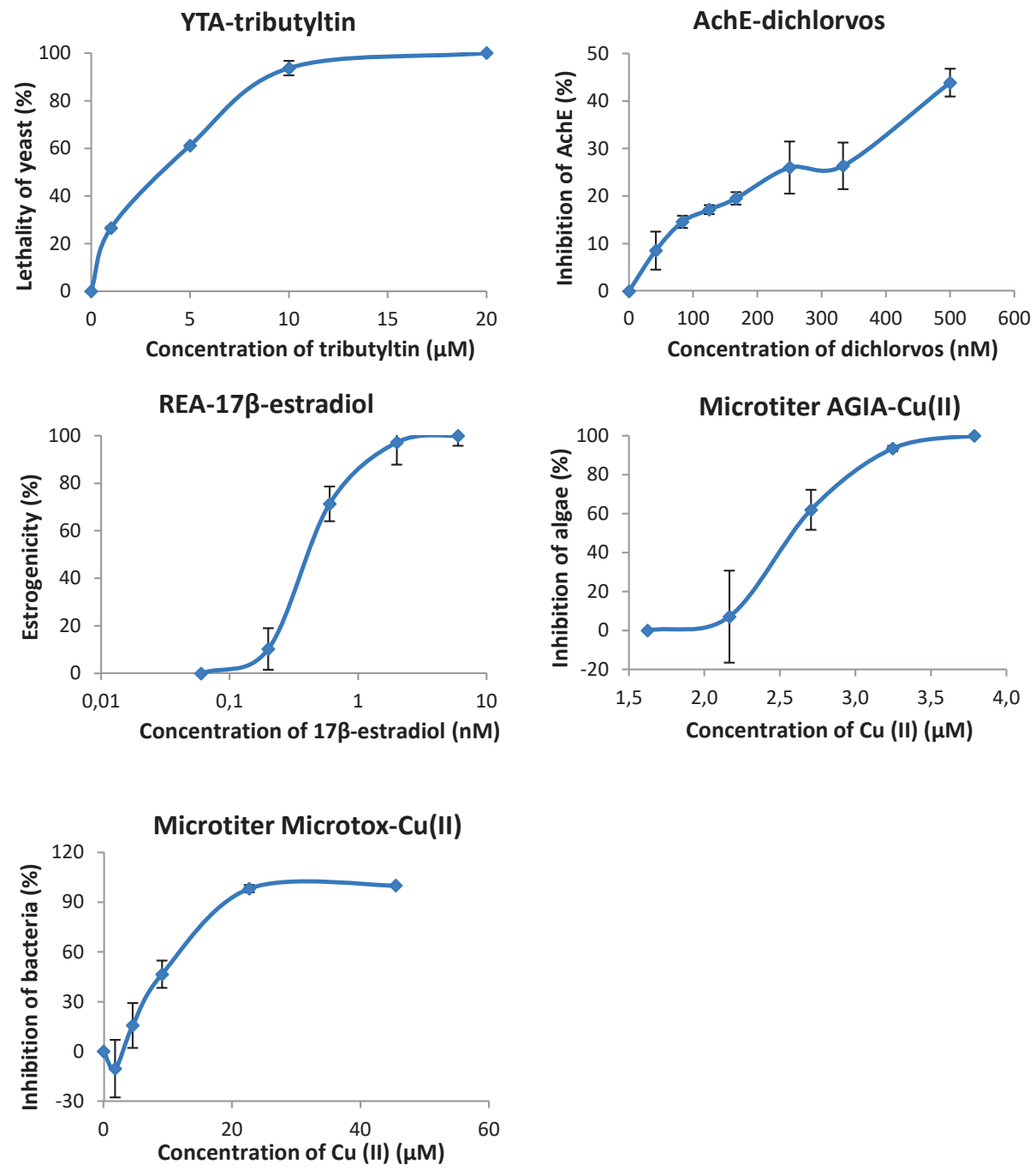

Figure S2.2 Standard curves of reference compounds in five bioanalyses. Values of Axis x are the concentrations of standard compounds in wells. Results are shown as average and standard error $(n=5$ in microtiter AGIA; $\mathrm{n}=3$ in the other bioanalyses). 


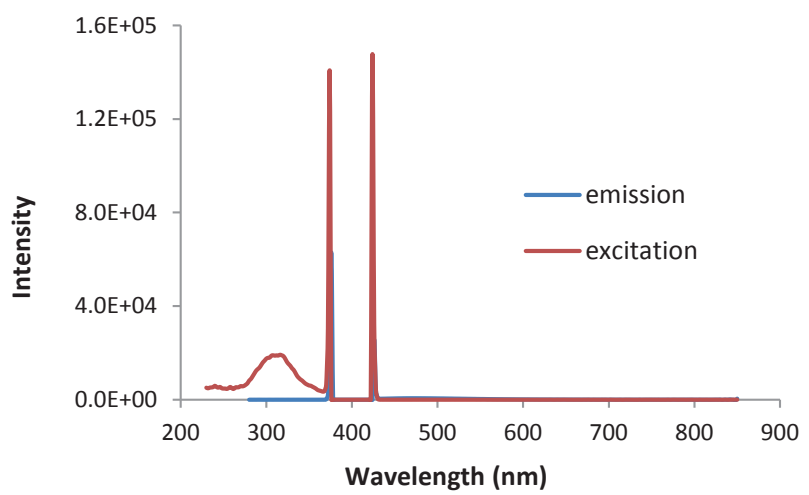

Figure S2.3 Fluorescence scans of the wastewater extract. Results showed that maximum fluorescence-related wavelength did not overlap with the measured wavelengths in bioanalyses as shown in Table S2.6.

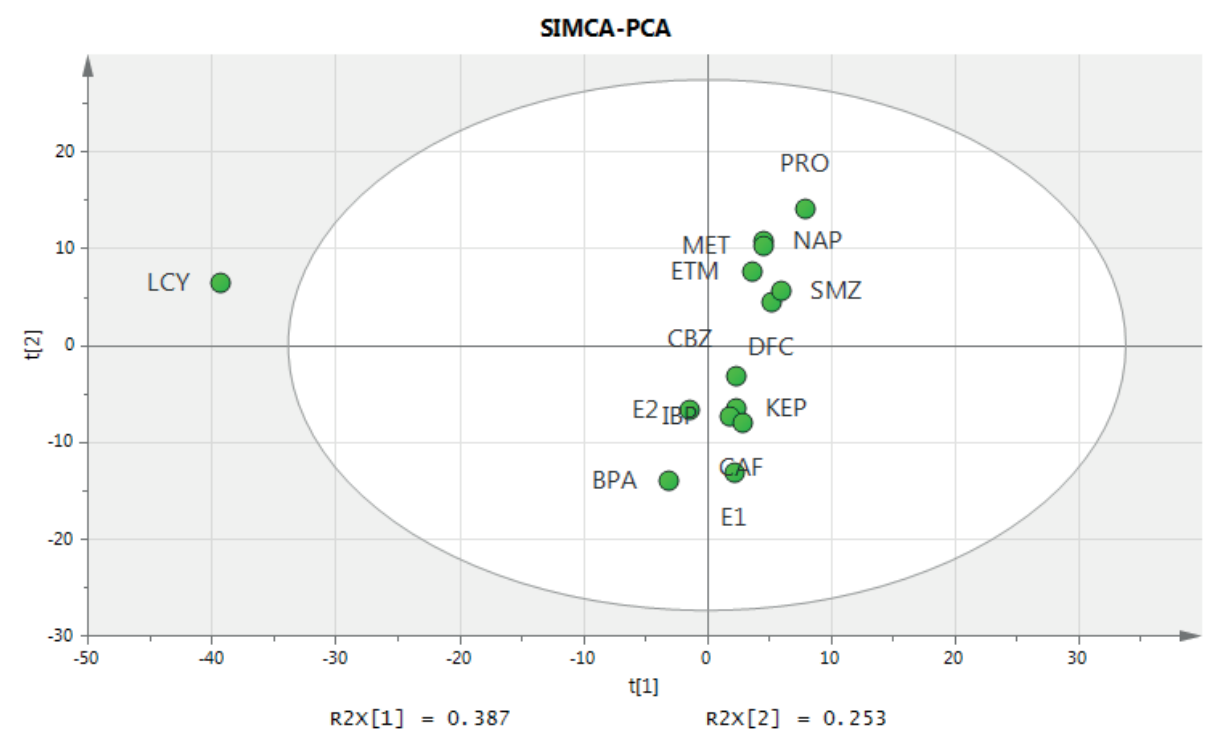

Figure S2.4 Principle component analysis of the relationship between removal efficiency of PhACs and their physicochemical properties, including $\mathrm{pKa}, \log \mathrm{K}_{\text {ow }}$ and $\log \mathrm{D}_{\text {ow }}$ (Table S2.1). No clear correlation was found because $\mathrm{PhACs}$ with similar properties did not cluster. 

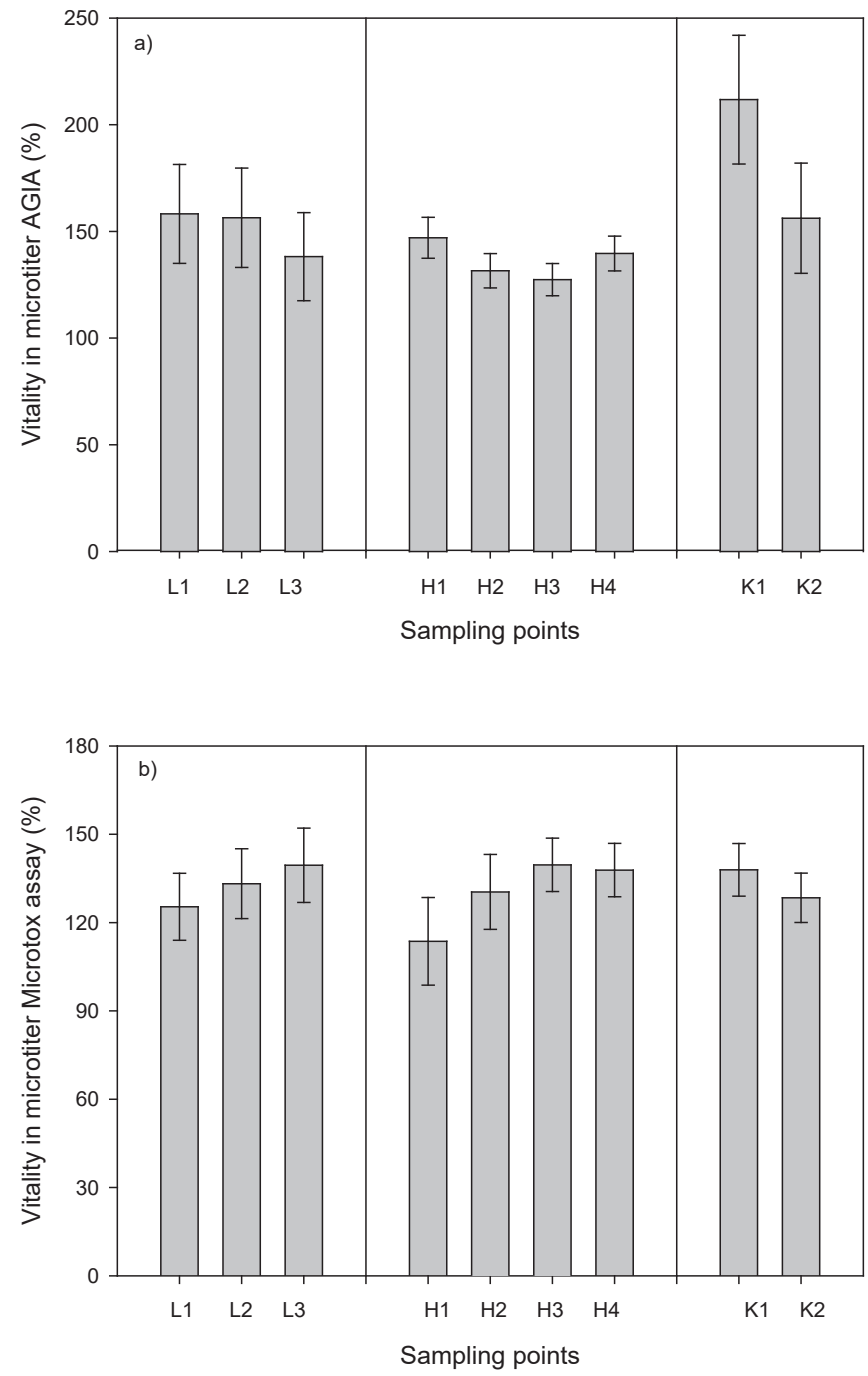

Figure S2.5 Toxic potency of sample extracts in different sampling points in two different bioanalyses: a) Microtiter algal growth inhibition assay (AGIA); b) Microtiter Microtox assay. Toxicity data are mean value \pm standard error $(n=6)$. 


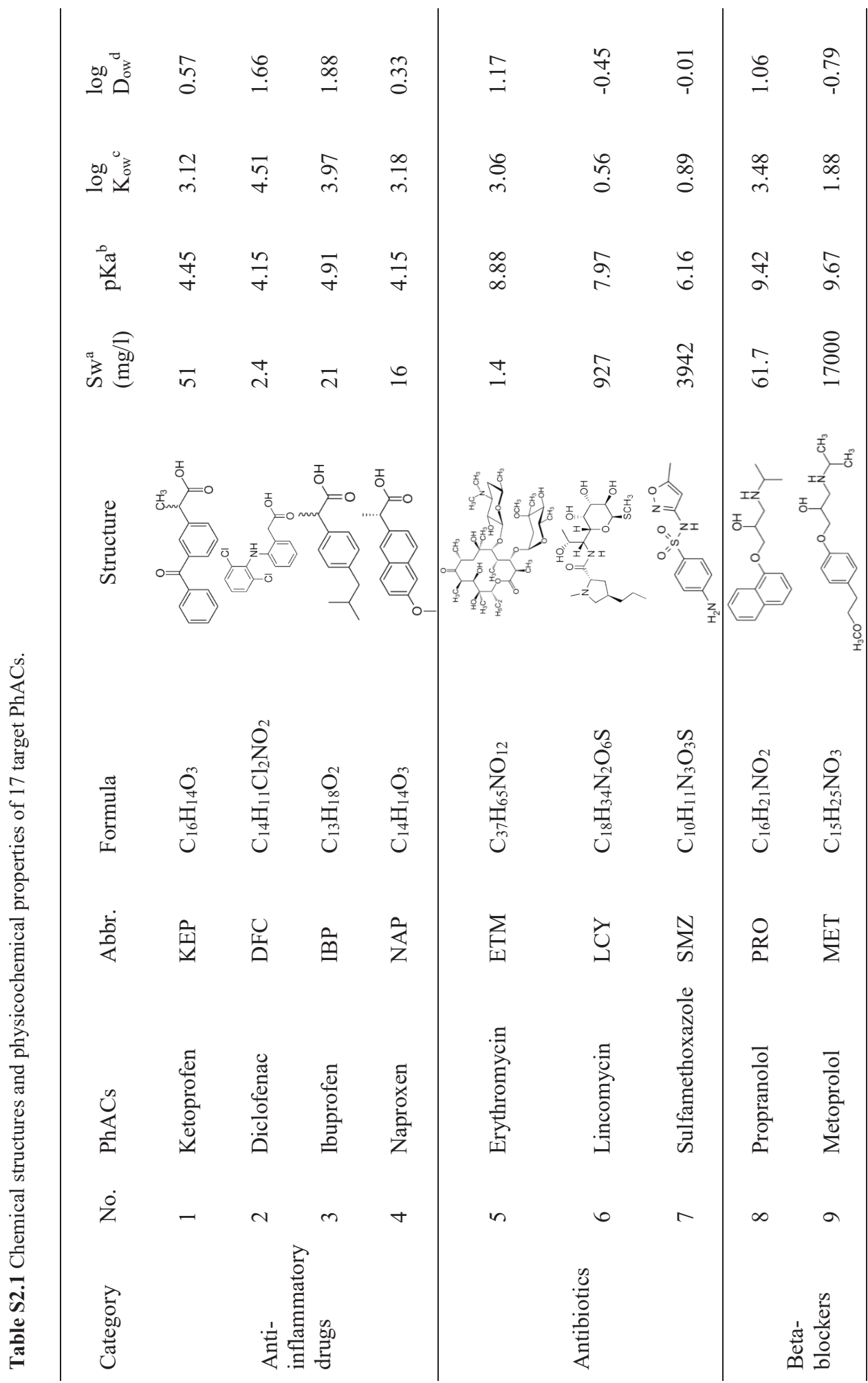


Chapter 2

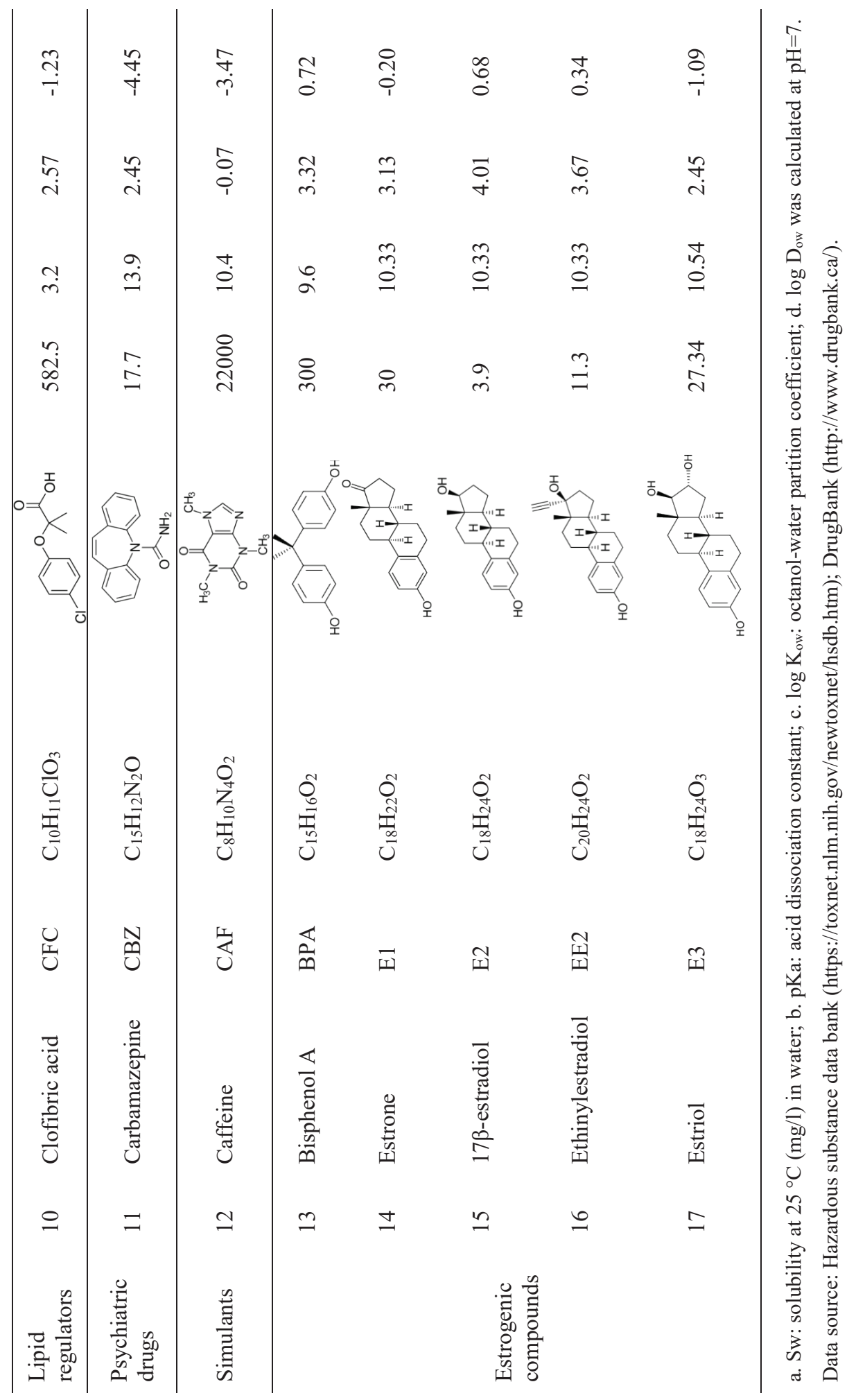


Table S2.2 Overview and operational parameters of target WWTPs and their CWs.

\begin{tabular}{|c|c|c|c|}
\hline Parameters & $\begin{array}{l}\text { Land van Cuijk } \\
\text { (CW-L) }\end{array}$ & $\begin{array}{l}\text { Hapert } \\
(\mathrm{CW}-\mathrm{H})\end{array}$ & $\begin{array}{l}\text { Kaatsheuvel } \\
(\mathrm{CW}-\mathrm{K})\end{array}$ \\
\hline $\begin{array}{l}\text { Capacity } \\
\text { (inhabitant equivalent) }\end{array}$ & 175,000 & 71,000 & 57,300 \\
\hline Wastewater source & $\begin{array}{l}43 \% \text { domestic, } 57 \% \\
\text { industrial }^{\mathrm{a}} \& \text { hospital }\end{array}$ & $\begin{array}{l}78 \% \text { domestic, } 22 \% \text { industrial }^{\mathrm{c}}\end{array}$ & Domestic \\
\hline Biological treatment & $\begin{array}{l}\text { Activated sludge with } \\
\text { sand filter }\end{array}$ & Oxidation ditch & $\begin{array}{l}\text { Oxidation ditch } \\
\text { with sand filter }\end{array}$ \\
\hline $\begin{array}{l}\text { Flow rate of WWTPs } \\
\left(\mathrm{m}^{3} / \mathrm{h}\right)\end{array}$ & 2,500 & 718 & 2,200 \\
\hline Effluent treated by CWs & Approx. 25\% & Approx. $15 \%$ & Approx.10\% \\
\hline Type of CWs & $\begin{array}{l}\text { Surface flow, } \\
\text { since } 1999\end{array}$ & $\begin{array}{l}\text { Surface flow, } \\
\text { since } 2001\end{array}$ & $\begin{array}{l}\text { Vertical subsurface } \\
\text { flow, since } 1997\end{array}$ \\
\hline Area of CWs $\left(\mathrm{m}^{2}\right)$ & 20,000 & 7,009 & 7,800 \\
\hline Flow rate of CWs $\left(\mathrm{m}^{3} / \mathrm{h}\right)$ & 360 & 300 & 58 \\
\hline $\begin{array}{l}\text { Hydraulic retention time } \\
\text { of CWs (d) }\end{array}$ & 4 & 0.82 & 1.7 \\
\hline $\begin{array}{l}\text { Hydraulic loading rate of } \\
\mathrm{CWs}(\mathrm{cm} / \mathrm{d})\end{array}$ & 43.3 & 102.7 & 17.6 \\
\hline Plant species & Phragmites australis & $\begin{array}{l}\text { Phragmites australis } \\
\text { (reed bed); } \\
\text { trees (swamp) }\end{array}$ & $\begin{array}{l}\text { Phragmites } \\
\text { australis }\end{array}$ \\
\hline Receiving water & River Maas & River Grote Beerze & Lake Ven west \\
\hline
\end{tabular}

a. Sources of industrial wastewater are industrial process water, organic biodegradable wastewater, and paper manufacturing wastewater. b. Two small hospitals are connected to the sewage system of WWTP Land van Cuijk. The exact individual percentage of industries and hospitals is unknown; c. Main sources of industrial wastewater are from meat processing industries, metal industries, and food industries. 
Table S2.3 Recovery rate of individual PhACs in deionized water and wastewater effluent.

\begin{tabular}{llll}
\hline \multirow{2}{*}{ Category } & Compounds & \multicolumn{2}{c}{ Recovery (\%) } \\
& & Deionized water & Wastewater \\
\hline \multirow{3}{*}{ Anti-inflammatory drugs } & DFC & $128-129$ & $109-115$ \\
& IBP & $103-116$ & $1-2$ \\
& NAP & $154-158$ & $116-132$ \\
\hline \multirow{3}{*}{ Antibiotics } & ETM & $159-185$ & $108-112$ \\
& LCY & $13-38$ & $155-181$ \\
\hline \multirow{2}{*}{ Beta-blockers } & SMZ & $91-91$ & $83-83$ \\
& PRO & $53-56$ & $93-93$ \\
\hline Lipid regulators & MET & $3-10$ & $71-100$ \\
\hline Psychiatric drugs & CFC & $83-99$ & $144-147$ \\
\hline Simulants & CBZ & $94-99$ & $15-17$ \\
\hline Internal standard & CAF & $81-94$ & $85-89$ \\
\hline \multirow{2}{*}{ Estrogenic compounds } & Dihy-CBZ & $115-117$ & $130-131$ \\
& BPA & 19.0 & 17.0 \\
\hline Internal standard & E1 & $78-80$ & $45-71$ \\
\hline & E2 & $101-110$ & $48-49$ \\
\hline & EE2 & $94-96$ & $61-62$ \\
& E3 & $96-99$ & $42-64$ \\
& E2-d3 & $90-106$ & $44-52$ \\
\hline
\end{tabular}

a. Dihydrocarbamazepine, the internal standard for UHPLC-MS/MS analysis. b. The internal standard for GC-MS/MS analysis

Table S2.4 Operational parameters of PhACs analysis on GC-MS/MS.

\begin{tabular}{lllllll}
\hline \multirow{2}{*}{ Segment } & Compounds & \multirow{2}{*}{$\begin{array}{c}\text { Retention time } \\
(\mathrm{min})\end{array}$} & \multicolumn{4}{c}{ Parent and product ion (from high to low) } \\
\cline { 4 - 7 } & & & 1 & $\mathrm{CE}(\mathrm{v})^{\mathrm{a}}$ & 2 & CE (v) \\
\hline 1 & BPA & 9.616 & $372.3>357.3$ & 15 & $372.3>191.1$ & 32 \\
4 & E1 & 14.656 & $414.3>155.3$ & 16.5 & $414.3>296.5$ & 7 \\
3 & E2 & 14.748 & $416.3>285.3$ & 7 & $416.3>326.3$ & 6 \\
4 & EE2 & 16.228 & $425.3>193.2$ & 14 & $425.3>231.2$ & 15 \\
5 & E3 & 16.456 & 504.4 & 414.3 & 386.3 & 311.2 \\
6 & E2-d3 & 14.707 & $419.3>285.3$ & 7 & $/$ & $/$ \\
\hline
\end{tabular}

a. $\mathrm{CE}=$ collision energy. $\mathrm{b}$. Estriol was analysed in selected ion monitoring (SIM) mode. 
Evaluation of field constructed wetlands

Table S2.5 Operational parameters of PhACs analysis on UHPLC-MS/MS.

\begin{tabular}{llllllll}
\hline Compounds & Mode & $\begin{array}{l}\text { Rentition } \\
\text { time }(\mathrm{min})\end{array}$ & Parent ion & CE (v) & $\begin{array}{l}\text { product } \\
\text { ion-1 }\end{array}$ & $\begin{array}{l}\text { product } \\
\text { ion-2 }\end{array}$ & $\begin{array}{l}\text { product } \\
\text { ion-3 }\end{array}$ \\
\hline KEP & - & 7.5 & 253 & 10 & 209 & 197 & $\mathbf{1 0 5}^{\text {a }}$ \\
DFC & - & 8.24 & 294 & 10 & $\mathbf{2 5 0}$ & 214 & \\
IBP & - & 1.33 & 205 & 10 & $\mathbf{1 6 1}$ & 159 & \\
NAP & - & 7.63 & 229 & 10 & $\mathbf{1 8 5}$ & 169 & \\
ETM & + & 6.86 & 734 & 20 & 558 & 576 & $\mathbf{1 5 8}$ \\
LCY & + & 3.81 & 407 & 29 & $\mathbf{3 5 9}$ & 126 & \\
SMZ & + & 4.51 & 254 & 15 & $\mathbf{1 8 8}$ & 156 & \\
PRO & + & 5.72 & 260 & 20 & $\mathbf{1 8 3}$ & 116 & \\
MET & + & 4.73 & 268 & 20 & $\mathbf{1 5 9}$ & 133 & \\
CFC & - & 7.5 & 213 & 10 & $\mathbf{1 2 7}$ & 85 & \\
CBZ & + & 6.95 & 237 & 20 & 194 & $\mathbf{1 9 2}$ & \\
CAF & + & 4.31 & 195 & 20 & 138 & $\mathbf{1 1 0}$ & \\
Dihy-CBZ & + & 7.15 & 239 & 20 & 195 & $\mathbf{1 8 0}$ & \\
\hline
\end{tabular}

a. Product ion in bold was selected for the quantification.

Table S2.6 Description of bioassays applied for bioanalysis of the toxic potency of wastewater extracts.

\begin{tabular}{|c|c|c|c|c|c|}
\hline Toxicity & Bioassays & Receptors & $\begin{array}{l}\text { Exposure } \\
\text { time }\end{array}$ & Measurement & $\begin{array}{l}\text { Reference } \\
\text { compound }\end{array}$ \\
\hline \multirow{4}{*}{ Acute } & YTA & $\begin{array}{l}\text { Saccharomyces } \\
\text { cerevisiae }\end{array}$ & $1 \mathrm{~h}$ & $\begin{array}{l}\text { Fluorescence } \\
\lambda_{\text {emission }}=590 \mathrm{~nm}, \\
\lambda_{\text {excitation }}=530 \mathrm{~nm}\end{array}$ & Tributyltin \\
\hline & AchE Assay & $\begin{array}{l}\text { Acetylcholinester } \\
\text { ase }\end{array}$ & $14 \min$ & $\begin{array}{l}\text { Absorbance } \\
\lambda=412 \mathrm{~nm}\end{array}$ & Dichlorvos \\
\hline & REA & $\begin{array}{l}\text { Genetically } \\
\text { modified baker's } \\
\text { yeast }\end{array}$ & $24 \mathrm{~h}$ & $\begin{array}{l}\text { Fluorescence } \\
\lambda_{\text {emission }}=530 \mathrm{~nm}, \\
\lambda_{\text {excitation }}=485 \mathrm{~nm}\end{array}$ & $\begin{array}{l}17 \beta- \\
\text { estradiol }\end{array}$ \\
\hline & $\begin{array}{l}\text { Microtiter Microtox } \\
\text { Assay }\end{array}$ & Vibrio fischeri & $15 \mathrm{~min}$ & Luminescence & $\mathrm{CuSO}_{4}$ \\
\hline Chronic & Microtiter AGIA & $\begin{array}{l}\text { Pseudokirchneriel } \\
\text { la subcapitata }\end{array}$ & $72 \mathrm{~h}$ & $\begin{array}{l}\text { Fluorescence } \\
\lambda_{\text {emission }}=680 \mathrm{~nm}, \\
\lambda_{\text {excitation }}=435 \mathrm{~nm}\end{array}$ & $\mathrm{CuSO}_{4}$ \\
\hline
\end{tabular}


Table S2.7 Overview of primer sequencing and thermal cycling conditions for qPCR.

\begin{tabular}{|c|c|c|c|c|}
\hline ARGs & Sequence (5'-3') & Thermal profile & Cycles & $\begin{array}{l}\text { Detection } \\
\text { format }\end{array}$ \\
\hline \multirow{2}{*}{$16 S[126]$} & ACTCCTACGGGAGGGCAG & $95^{\circ} \mathrm{C} 3 \mathrm{~min}$ & 1 & SYBR \\
\hline & GACTACCAGGGTATCTAATCC & $95^{\circ} \mathrm{C} 15 \mathrm{~s}, 60^{\circ} \mathrm{C} 45 \mathrm{~s}$ & 40 & Green \\
\hline \multirow[t]{2}{*}{ intl1 [23] } & GCCTTGATGTTACCCGAGAG & $95^{\circ} \mathrm{C} 3 \mathrm{~min}$ & 1 & TaqMan \\
\hline & GATCGGTCGAATGCGTGT & $95^{\circ} \mathrm{C} 15 \mathrm{~s}, 60^{\circ} \mathrm{C} 45 \mathrm{~s}$ & 40 & \\
\hline \multirow[t]{2}{*}{ sul $1[166]$} & CCGTTGGCCTTCCTGTAAAG & $95^{\circ} \mathrm{C} 3 \min$ & 1 & TaqMan \\
\hline & TTGCCGATCGCGTGAAGT & $95^{\circ} \mathrm{C} 15 \mathrm{~s}, 60^{\circ} \mathrm{C} 45 \mathrm{~s}$ & 40 & \\
\hline \multirow[t]{2}{*}{ sul 2 [167] } & CGGCTGCGCTTCGATT & $95^{\circ} \mathrm{C} 3 \mathrm{~min}$ & 1 & TaqMan \\
\hline & CGCGCGCAGAAAGGATT & $95^{\circ} \mathrm{C} 15 \mathrm{~s}, 60^{\circ} \mathrm{C} 45 \mathrm{~s}$ & 40 & \\
\hline \multirow[t]{2}{*}{$\operatorname{ermB}[203]$} & AAAACTTACCCGCCATACCA & $95^{\circ} \mathrm{C} 3 \mathrm{~min}$ & 1 & SYBR \\
\hline & TTTGGCGTGTTTCATTGCTT & $95^{\circ} \mathrm{C} 15 \mathrm{~s}, 60^{\circ} \mathrm{C} 45 \mathrm{~s}$ & 40 & Green \\
\hline
\end{tabular}

Table S2.8 Physicochemical analysis of collected wastewater samples and removal efficiencies in CWs (average \pm std, $\mathrm{n}=2$ ).

\begin{tabular}{|c|c|c|c|c|c|c|c|c|}
\hline WWTPs & Sites & $\begin{array}{c}\mathrm{DO} \\
(\mathrm{mg} / \mathrm{l})\end{array}$ & $\mathrm{pH}$ & $\mathrm{T}\left({ }^{\circ} \mathrm{C}\right)$ & $\begin{array}{l}\text { COD } \\
(\mathrm{mg} / \mathrm{l})\end{array}$ & $\begin{array}{l}\mathrm{NH}_{4}-\mathrm{N} \\
(\mathrm{mg} / \mathrm{l})\end{array}$ & $\begin{array}{l}\mathrm{NO}_{3}-\mathrm{N} \\
(\mathrm{mg} / \mathrm{l})\end{array}$ & $\begin{array}{c}\mathrm{TP} \\
(\mathrm{mg} / \mathrm{l})\end{array}$ \\
\hline \multirow{3}{*}{ CW-L } & L1 & 8.1 & 6.4 & 22.6 & $29.4 \pm 0.28$ & 0.05 & 2.69 & $0.38 \pm 0.01$ \\
\hline & L2 & 1.2 & 6.5 & 20.3 & $\begin{array}{c}32.70 \pm 0.85 \\
(-11)^{\mathrm{a}}\end{array}$ & $\begin{array}{c}0.64 \pm 0.01 \\
(-1180)\end{array}$ & $\begin{array}{l}0.91 \pm 0.01 \\
\quad(66)\end{array}$ & $\begin{array}{c}0.30 \pm 0.03 \\
(21)\end{array}$ \\
\hline & L3 & 16.2 & 7.1 & 24.6 & $35.35 \pm 0.78$ & 0.02 & $0.28 \pm 0.02$ & $0.13 \pm 0.01$ \\
\hline \multirow{4}{*}{ CW-H } & H1 & 5.7 & 6.5 & 22.5 & $28.65 \pm 1.48$ & $0.70 \pm 0.01$ & 0.4 & $0.38 \pm 0.17$ \\
\hline & $\mathrm{H} 2$ & 5.7 & 6.7 & 22.4 & $31.70 \pm 1.27$ & 0.7 & $0.54 \pm 0.05$ & $0.30 \pm 0.03$ \\
\hline & $\mathrm{H} 3$ & 5.9 & 6.8 & 22.9 & $29.95 \pm 0.07$ & $0.70 \pm 0.01$ & $0.52 \pm 0.01$ & 0.28 \\
\hline & $\mathrm{H} 4$ & 5.5 & 6.7 & 21.6 & $\begin{array}{c}30.15 \pm 2.05 \\
\quad(5)\end{array}$ & $\begin{array}{c}0.54 \pm 0.01 \\
(23)\end{array}$ & $\begin{array}{c}0.78 \pm 0.01 \\
(-44)\end{array}$ & $\begin{array}{c}0.40 \pm 0.04 \\
(-33)\end{array}$ \\
\hline \multirow{2}{*}{ CW-K } & $\mathrm{K} 1$ & 9.4 & 6.8 & 22.7 & $19.85 \pm 0.92$ & 0.02 & $1.18 \pm 0.01$ & $0.08 \pm 0.01$ \\
\hline & $\mathrm{K} 2$ & 8.4 & 6.8 & 21.9 & $\begin{array}{c}14.70 \pm 4.10 \\
(26)\end{array}$ & $\begin{array}{c}0.02 \\
(0) \\
\end{array}$ & $\begin{array}{c}0.74 \pm 0.01 \\
(37) \\
\end{array}$ & $\begin{array}{l}0.12 \\
(-50)\end{array}$ \\
\hline $\begin{array}{l}\text { EU standard } \\
{[96]}\end{array}$ & I & / & I & I & $<125$ & \multicolumn{2}{|c|}{$\begin{array}{l}\text { Total nitrogen }(\mathrm{TN}) \\
<15(10,000-100,000 \\
\text { p.e. }) \\
<10(>100,000 \text { p.e. })\end{array}$} & $\begin{array}{l}<2(10,000- \\
100,000 \text { p.e. }) \\
<1(> \\
100,000 \text { p.e. })\end{array}$ \\
\hline
\end{tabular}

a. Removal efficiencies in three CWs are displayed in brackets and were calculated based on L1 to L2, $\mathrm{H} 2-\mathrm{H} 4$, and K1-K2, respectively. 


\section{Evaluation of field constructed wetlands}

Table S2.9 Removal of PhACs in three CWs (average \pm std, $n=2$ ). Negative numbers mean the levels of the compounds increased over the CW.

\begin{tabular}{cccc}
\hline \multirow{2}{*}{ PhACs } & \multicolumn{3}{c}{ Removal efficiency (\%) } \\
\cline { 2 - 4 } & CW-L & CW-H & CW-K \\
\hline KEP & ND & $-7.3 \pm 57.9$ & ND \\
DFC & $32.8 \pm 24.8$ & $8.4 \pm 10.5$ & $73.1 \pm 11$ \\
IBP & $-11.0 \pm 22.5$ & $-33.4 \pm 11.3$ & $57.7 \pm 1.1$ \\
NAP & $40.6 \pm 7.1$ & $4.4 \pm 7.6$ & $44.3 \pm 2.3$ \\
ETM & $-7.2 \pm 5.3$ & $6.3 \pm 12.2$ & $41.2 \pm 0$ \\
LCY & $-31.0 \pm 0$ & $-534.1 \pm 86.9$ & $76.3 \pm 1.2$ \\
SMZ & $5.6 \pm 7.4$ & $12.7 \pm 17.1$ & $82.8 \pm 9.8$ \\
PRO & $36.3 \pm 34.3$ & $-0.9 \pm 40.9$ & $84.9 \pm 1.1$ \\
MET & $-23.6 \pm 6.8$ & $5.2 \pm 1.2$ & ND \\
CFC & ND & ND & $-27.4 \pm 0.7$ \\
CBZ & $1.5 \pm 5.6$ & $-2.8 \pm 1.9$ & $-56.4 \pm 37.9$ \\
CAF & $-15.3 \pm 16.9$ & $-2 \pm 2.2$ & $-65.7 \pm 61.2$ \\
BPA & $-29.2 \pm 116.3$ & $-88.3 \pm 78.2$ & ND \\
E1 & ND & ND & ND \\
E2 & ND & $-70.2 \pm 17.4$ & ND \\
EE2 & ND & ND & ND \\
E3 & ND & ND & \\
\hline
\end{tabular}

Notes: Ellipse frames represent removal higher than $75 \%$; rectangle frames represent removal higher between $30-60 \%$. ND = not determined because levels were below the limit of detection (Table 2.1).

Table S2.10 Removal detected genes in the three CWs.

\begin{tabular}{lllll}
\hline CWs & Genes & $\begin{array}{l}\text { Influent } \\
(\text { copies } / \mathrm{mL})\end{array}$ & $\begin{array}{l}\text { Effluent } \\
\text { copies/mL) }\end{array}$ & $\begin{array}{l}\text { Removal } \\
(\%)\end{array}$ \\
\hline \multirow{6}{*}{ CW-L } & sull & $7.5 \times 10^{6}$ & $2.3 \times 10^{7}$ & -207.7 \\
& sul2 & $1.2 \times 10^{3}$ & $2.7 \times 10^{3}$ & 66.7 \\
& ermB & $2.8 \times 10^{4}$ & $6.9 \times 10^{2}$ & 95.4 \\
& Intl1 & $2.9 \times 10^{4}$ & $4.9 \times 10^{0}$ & 82.3 \\
& I6S & $4.7 \times 10^{6}$ & $2.5 \times 10^{4}$ & 13.8 \\
& sul1 & $4.6 \times 10^{4}$ & $3.4 \times 10^{7}$ & -619.8 \\
CW-H & sul2 & $7.3 \times 10^{3}$ & $1.7 \times 10^{5}$ & -261.5 \\
& ermB & $2.4 \times 10^{2}$ & $2.2 \times 10^{4}$ & -206.5 \\
& Intl1 & $7.8 \times 10^{4}$ & $7.3 \times 10^{1}$ & 69.8 \\
& $16 S$ & $8.4 \times 10^{5}$ & $3.2 \times 10^{5}$ & -308.9 \\
& sul1 & $6.4 \times 10^{2}$ & $1.3 \times 10^{6}$ & -60.0 \\
CW-K & sul2 & $1.0 \times 10^{2}$ & $7.0 \times 10^{1}$ & 89.1 \\
& ermB & $9.0 \times 10^{0}$ & $3.0 \times 10^{1}$ & 70.5 \\
& Intl1 & $2.4 \times 10^{3}$ & ND & 100 \\
\hline
\end{tabular}

a. $\mathrm{ND}=$ not determined because levels were below the limit of detection 4.81 copies $/ \mathrm{ml}$. 
Sunlight

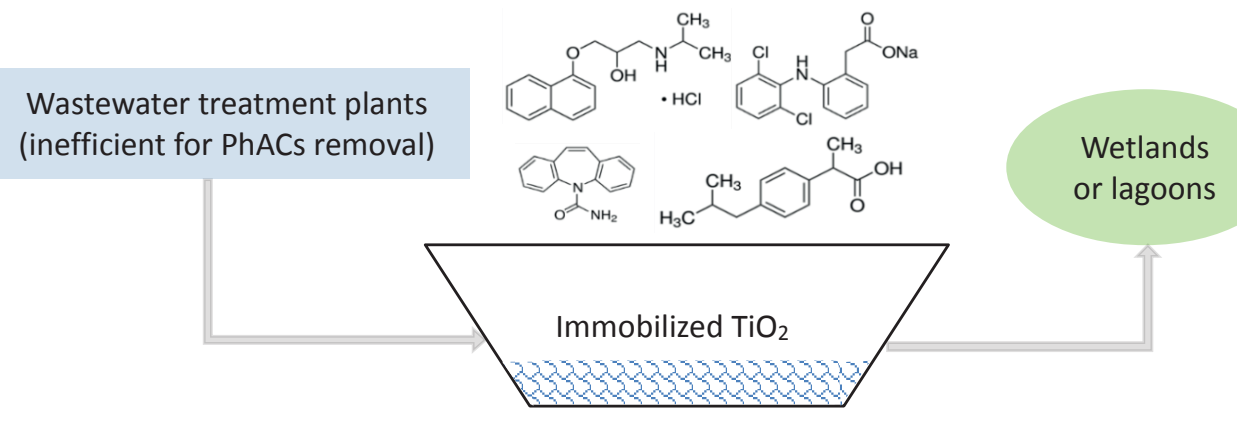

Open shallow cell 


\section{Chapter 3}

Degradation of pharmaceuticals in wastewater using immobilized $\mathrm{TiO}_{2}$ photocatalysis under simulated solar irradiation

A modified version of this chapter was published as

He, Y., Sutton, N.B., Rijnaarts, H.H.M., Langenhoff, A.A.M. Degradation of pharmaceuticals in wastewater using immobilized $\mathrm{TiO}_{2}$ photocatalysis under simulated solar irradiation. Applied Catalysis B: Environmental 2016, 182, 132-141. 


\title{
Photodegradation
}

\begin{abstract}
Pharmaceutically active compounds (PhACs) are not efficiently removed in wastewater treatment plants and are released into surface waters resulting in toxin accumulation. The aims of this study were to investigate the effect of solar irradiation on PhACs in wastewater using immobilized $\mathrm{TiO}_{2}$ present as a catalyst, and to study the potential of this photocatalysis technique as a post-treatment process for wastewater effluent. We treated a mixture of PhACs spiked in wastewater effluent and in deionized water as a control with simulated solar irradiation for $96 \mathrm{~h}$. Experiments were conducted with immobilized $\mathrm{TiO}_{2}$ (photocatalysis) and without (photolysis). First, $\mathrm{TiO}_{2}$ was successfully immobilized on $200-500 \mu \mathrm{m}$ sand by using a sol-gel method. The photocatalysis resulted in high removal efficiencies for poorly biodegradable $\mathrm{PhACs}$ in wastewater effluent: $100 \%$ for propranolol, $100 \%$ for diclofenac, and $76 \pm 3 \%$ for carbamazepine. Photodegradation of all four PhACs followed pseudo-firstorder kinetics, and the kinetic constant of photocatalysis was much higher than that of photolysis in the absence of a catalyst. Natural dissolved organic matter (DOM) in wastewater effluent enhanced photodegradation of $\mathrm{PhACs}$ by producing reactive radicals. However, at the same time, DOM inhibited photodegradation, possibly because DOM reforms the oxidation intermediates of PhACs into parent compounds. From an application perspective, water depth was confirmed as a key factor in photodegradation of $\mathrm{PhACs}$ due to light attenuation by modelling and experimental results. In addition, after photocatalysis, toxicity of PhACs decreased and biodegradability of wastewater effluent increased slightly. In conclusion, the technique is a promising post-treatment process to improve water quality, prior to discharging to natural waters or to polishing water treatment systems such as wetlands and lagoons.
\end{abstract}

\section{Keywords}

Pharmaceuticals; Photocatalysis; Sunlight; DOM; Application 


\subsection{Introduction}

Many pharmaceutically active compounds (PhACs) behave as persistent organic micropollutants, as evidenced by their continuous input and accumulation in the environment [252]. PhACs are released into the aquatic environment, primarily due to their incomplete removal by conventional treatment processes in wastewater treatment plants (WWTPs) [144]. PhACs are evidenced or suspected to provoke toxic effects on living organisms even when present at concentrations as low as ng/l level [127]. Consequently, the accumulation of $\mathrm{PhACs}$ poses a threat to the quality of water resources. Advanced technologies such as application of UV and ozonation are effective for treating some PhACs, but have limited applicability due to high construction, maintenance and energy costs [261]. Therefore, more efficient treatment techniques need to be explored and further developed to eliminate PhACs emissions to the environment.

Multiple biotic and abiotic routes could transform PhACs once they reach the surface water [208]. One of the abiotic processes is photodegradation, which is a significant and predominant pathway to remove PhACs in natural water treatment systems such as wetlands, ponds and lagoons [10]. In addition, photodegradation has been considered as an effective remediation tool for wastewater, especially for increasing the biodegradability of contaminants and detoxifying the effluent stream [284]. In order to maximize photodegradation performance, photocatalysis has often been combined with solar irradiation, which could provide a more economically feasible and ecologically effective alternative, as compared to using artificial light sources. Photolysis and photocatalysis of PhACs have been investigated previously, mostly in pure water or surface water matrices under UV irradiation [52, 289, 290, 341]. However, to our knowledge, PhACs removal by both photolysis and photocatalysis from wastewater effluent under solar irradiation has only been studied by a few groups [201, 275].

In our study, we focused on PhACs that are most commonly detected in wastewater affected water systems and aquatic organisms [146, 306], and are not easily biodegradable: propranolol (PRO, $\beta$-blocker), carbamazepine (CBZ, antiepileptics), ibuprofen and diclofenac (IBP and DFC, anti-inflammatories and analgesics). For example, removal efficiencies of CBZ and DFC in WWTPs have been reported below $30 \%$ and $21-40 \%$ respectively [408]. $\mathrm{TiO}_{2}$ has been most extensively used in photocatalytic reactions because it is biologically and chemically inert, inexpensive, and non-toxic [55]. In addition, at sea-level heights of the 


\section{Photodegradation}

surface of the Earth, the sun could still provide sufficient light intensity and available spectrum to activate $\mathrm{TiO}_{2}$ [91]. However, $\mathrm{TiO}_{2}$ nanoparticles may exert ecotoxicological effects on aquatic microorganisms. For example, the half maximal effective concentration (EC50) of $\mathrm{TiO}_{2}(25 \mathrm{~nm})$ was determined about $40 \mathrm{mg} / \mathrm{l}$ for algae [179]. According to the EU Directive on classification, packaging and labelling of dangerous substances [107], a toxicity in the range of $10-100 \mathrm{mg} / 1$ is classified as harmful to aquatic organisms; and may cause long-term adverse effects in the aquatic environment. Thus a non-dispersed method is needed when applying $\mathrm{TiO}_{2}$ in open water treatment systems. To this end, we immobilized $\mathrm{TiO}_{2}$ on sand to avoid this potential of aquatic system pollution. Previously, Avisar et al. [18] reported a $35 \%$ reduction of CBZ in surface water and negligible removal in WWE, when the matrices exposed to solar irradiation simulated by a $150 \mathrm{~W}$ Xenon arc lamp in the presence of nitrogen-doped $\mathrm{TiO}_{2}$-coated glass slides.

In this study, we aim to evaluate the application of photocatalysis for PhACs removal in WWE by combing solar light and immobilized $\mathrm{TiO}_{2}$. To determine the feasibility of such a system, and for proper design of experiments, we analysed the light attenuation at various water depths and its effect on photolysis efficiency of PhACs mathematically and experimentally. Based on the optimized depth, we investigated the reaction mechanisms of target PhACs spiked in WWE under simulated solar irradiation. Additionally, in order to test the potential application of the photocatalysis, we analysed the change of PhACs in toxicity and biodegradability during photodegradation. The outcome of this work contributes to understanding the solar induced photocatalysis as a low-cost, efficient treatment technique to remove PhACs as a post-WWTP process, before wastewater effluent is discharged into surface waters, such as lakes, rivers, natural wetlands, ponds, and lagoons.

\subsection{Materials and methods}

\subsubsection{Chemicals}

Chemicals were purchased from Sigma-Aldrich (USA) at various purity grades: $( \pm$ )Propranolol hydrochloride ( $\geq 99 \%$ ), diclofenac sodium salt ( $\geq 99 \%)$, carbamazepine $(\geq 99 \%)$, and ibuprofen ( $\geq 98 \%$ ). Physical and chemical characteristics of target PhACs is shown in Table S2.1. Fenoprofen calcium salt $(\geq 97 \%)$ and hydrate 10, 11-dihydrocarbamazepine $(\geq 99 \%)$ were used as internal standards (Sigma-Aldrich, USA). Acetonitrile with $0.1 \%$ formic acid, water with $0.1 \%$ formic acid, and methanol (Biosolve B.V., the Netherlands) 
were used for ultra-performance liquid chromatography (UPLC) analysis. Titanium (IV) butoxide ( $\geq 97 \%$, Sigma-Aldrich), $\mathrm{HNO}_{3}$ (Merck, Germany), ethanol ( $\geq 99 \%$, Merck) and glacial acetic acid (100\%, Merck) were used to immobilize the catalyst. All the other reagents used were of analytical grade or higher. Deionized water (DI) from a Milli-Q system (Millipore, Bedford, MA, USA) was used to prepare all solutions. All glassware was manually cleaned with detergent, acetone, DI, and air-dried before using.

\subsubsection{Immobilization techniques}

The purchased quartz sand (Happy home, the Netherlands) was already pre-rinsed by acid. The sand was sieved to obtain $200-500 \mu \mathrm{m}$ fractions, mixed with DI, stirred for 24 hours, and air-dried prior to being used as the immobilization substrate. $\mathrm{TiO}_{2}$ was coated on the sand by using the sol-gel method based on the work of Xia et al. [380]. Firstly, $14.2 \mathrm{ml}$ of Titanium (IV) butoxide was added to $50 \mathrm{ml}$ of ethanol and then the mixture was stirred continuously with a magnetic bar at $300 \mathrm{rpm}$ for $10 \mathrm{~min}$ (Heidolph MR 3001K, Germany). In this step, white precipitation should be avoided, otherwise the gel would not be formed. Secondly, $1 \mathrm{ml}$ of $0.1 \mathrm{~mol} / 1 \mathrm{HNO}_{3}, 1 \mathrm{ml}$ of DI and $1 \mathrm{ml}$ of glacial acetic acid were sequentially added dropwise. After stirring for 6-7 $\mathrm{min}$ at $300 \mathrm{rpm}$ at room temperature, a pale-yellow gel was formed. Next, $25 \mathrm{~g}$ sand, as the substrate for immobilization, was poured slowly into the gel and stirred for $15 \mathrm{~min}$ at $350 \mathrm{rpm}$. Finally, the gel mixture was dried for $2 \mathrm{~h}$ at $105^{\circ} \mathrm{C}$ in the oven (Heraeus Hanau, Germany). After totally cooling down in the oven, the 1-layer immobilized material was calcined for $3 \mathrm{~h}$ at $500^{\circ} \mathrm{C}$ and then cooled in the oven. The temperature of the oven was raised and cooled slowly before and after calcination to avoid cracking of the wrapped gel. The coating process was repeated twice more on the 1-layer immobilized sand to get 3-layers of immobilized material.

\subsubsection{Photodegradation experiments}

In order to simulate solar light, Xenon high-intensity discharge lamps (55W) with charger and battery were installed in a climate chamber (Heraeus Vötsch, Germany), in which the temperature was maintained at $25 \pm 2{ }^{\circ} \mathrm{C}$. Xe-lamps were fixed above the water surface and directly irradiated solutions. The lamps emitted irradiation in the wavelength range of 300 $800 \mathrm{~nm}$ and the irradiation intensity at the water surface was maintained at 1020 lux, which is comparable with the solar light intensity in the Netherlands (latitude $51^{\circ} 59^{\prime} 4$ '’ $\mathrm{N}$, longitude $5^{\circ} 39^{\prime} 31$ ' $E$, Figure S3.1) Light spectrum and intensity were detected by using a USB2000+ spectrometer (Ocean optics, UK). 


\section{Photodegradation}

In order to investigate the effect of light attenuation on the photodegradation of PhACs, a 0.5 $\mathrm{m}$ water filled column experiment was performed by adding $5 \mathrm{mg} / \mathrm{l}$ of each $\mathrm{PhAC}$ in DI and irradiating for $96 \mathrm{~h}$. The solution in the column was under static condition, and thus unhomogenized. Sampling points were evenly distributed on the side profile of the column so that water samples could be collected at every $0.1 \mathrm{~m}$ between depths of 0.0 to $0.5 \mathrm{~m}$. Prior to the start of the experiment, needles were affixed at each sampling point. Samples were collected by attaching a syringe to each needle and slowly withdrawing liquid, thus minimizing disruption of the water column during sampling. Samples were collected every $24 \mathrm{~h}$.

For the kinetic experiment, the initial concentration of individual PhACs was $5 \mathrm{mg} / \mathrm{l}$ spiked in $500 \mathrm{ml}$ DI or WWE, collected from Bennekom WWTP, the Netherlands. The basic water quality data of used WWE are shown in Table S3.1. WWE was filtrated by $0.4 \mu \mathrm{m}$ filter prior to being used in experiments. Photolysis experiments were performed by only applying irradiation, while photocatalysis experiments included 25 g immobilized $\mathrm{TiO}_{2}$. During the 96 h photodegradation, a shaking speed of $75 \mathrm{rpm}$ was kept so that the solution could be homogeneous while the catalyst was still stable at the bottom of the beaker. Adsorption experiments were performed in parallel in the dark by shielding light with foil and a sealed box. For all the experiments, the solution was shaken for $1 \mathrm{~h}$ to be totally homogeneous in darkness, prior to withdrawing the first sample as the initial concentration $\mathrm{C}_{0}$. Wastewater samples were centrifuged (IEC microlite centrifuge, Thermo, USA) at $10000 \mathrm{rmp}$ for $10 \mathrm{~min}$ before UPLC analysis. All the experiments were performed in duplicate.

\subsubsection{Analytical procedures}

\subsubsection{Basic chemical analysis}

Biodegradability was evaluated using the ratio between 5-day biochemical oxygen demand $\left(\mathrm{BOD}_{5}\right)$ and chemical oxygen demand $(\mathrm{COD})$. BOD 5 was determined by the OxiTop ${ }^{\circledR}$ system (WTW Weilheim, Germany) thermostated at $20{ }^{\circ} \mathrm{C}$. COD was measured using Dr. Lange test kits (Hach Lange GmbH, Germany) on a Hach DR/3900 spectrophotometer. The dissolved organic carbon and anion of WWE was measured using a Shimadzu analyser (Japan) and ion chromatography (Dionex ICS 2100, USA). WWE samples were freeze-dried into solid material by an alpha 2-4 $\mathrm{LD}^{+}$freeze dryer (Martin Christ $\mathrm{GmbH}$, Germany). FTIR analysis of $1 \mathrm{mg}$ WWE solid was performed using a Bruker Tensor 27 FTIR spectrometer equipped with a Platinum ATR accessory (Bruker, Germany). 


\subsubsection{Characterization of immobilization material}

Surface morphologies of raw sand and $\mathrm{TiO}_{2}$ coated sand were analysed by a field emission scanning electron microscope (SEM, Magellan 400, FEI, the Netherlands) with secondary electron detection at $2 \mathrm{kV}$, for which air-dried samples were attached on carbon adhesive tabs (EMS Washington USA) and subsequently sputter coated with $15 \mathrm{~nm}$ Iridium (SCD500 Leica, Vienna, Austria). Crystal structure of the sample was confirmed by X-ray diffraction (XRD, Philips, the Netherlands, $\mathrm{Cu}-\mathrm{K} \alpha, 30 \mathrm{kV}, 50 \mathrm{~mA}$ ). The stride length $A=1.5406 \lambda$ ( $\lambda$ is the

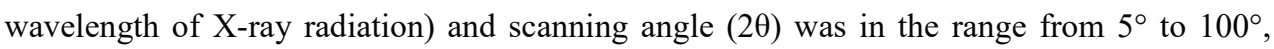
with a step size of $0.02^{\circ}$ and a measurement time of $1 \mathrm{~s}$ per step. Nitrogen gas adsorption isotherms were collected at $77 \mathrm{~K}\left(-196{ }^{\circ} \mathrm{C}\right)$ using a surface area and porosity analyzer (TriStar 3000, Micromeritics, USA). The Brunauer-Emmett-Teller (BET) model, BarrettJoyner-Halenda (BJH) model and t-plot model were used to determine the specific surface area, pore diameter, and micro-pore volume individually. Prior to collecting the isotherms, samples were degassed at $300{ }^{\circ} \mathrm{C}$ for two hours under a nitrogen gas flow of $30 \mathrm{~mL} / \mathrm{min}$ in a degassing apparatus (SmartPrep 065, Micromeritics, USA).

\subsubsection{UPLC analysis}

UPLC (ultimate 3000, Thermo, USA) with a diode array detector (DAD) was used to determine the concentration of PhACs. UPLC is equipped with CSH phenyl-Hexyl column $(1.7 \mu \mathrm{m}, 2.1 \times 150 \mathrm{~mm})$. The mobile phase was a mixture of A (water with $0.1 \%$ formic acid) and $\mathrm{B}$ (acetonitrile with $0.1 \%$ formic acid) with a flow rate at $0.3 \mathrm{ml} / \mathrm{min}$. The percentage of B gradually increased from $0 \%$ at $0.5 \mathrm{~min}$ to $80 \%$ at $13 \mathrm{~min}$, kept constant for $3 \mathrm{~min}$, then decreased to $0 \%$ at $17 \mathrm{~min}$ and stopped at $22.4 \mathrm{~min}$. The sample injection volume was $10 \mu \mathrm{l}$. Detection results were acquired and analysed automatically by software. Limits of detection of PhACs were $50 \mu \mathrm{g} / \mathrm{l}$ and detection results of the standards showed a good linearity $\left(\mathrm{R}^{2}>0.999\right)$.

\subsubsection{Toxicity assay}

Green algae, blue-green algae (cyanophyta) and luminescence bacteria, typical microorganisms, were exposed to PhACs samples collected at different irradiation time points to determine the acute and chronic toxicity of PhACs. Toxicity assays were completed based on the 96-wells plate experiment and detected by a plate reader (Tecan infinite M200 PRO, Switzerland). Green algae Pseudokirchneriella subcapitata and blue-green algae 


\section{Photodegradation}

Anabaena flos-aquae were obtained from Department of Aquatic Ecology and Water Quality Management, Wageningen University, the Netherlands; luminescence bacteria Vibrio fischeri was purchased from Microlan (the Netherlands). Algae were cultivated in an incubator shaker (Innova ${ }^{\circledR}$ 44, Eppendorf, USA, 600 lux, light/dark $=16$ h/8 h, $20^{\circ} \mathrm{C}, 80 \mathrm{rpm}$ ) and were fed with WC growth medium [148] weekly.

The growth inhibition of algae represents related toxicity of PhACs. Fluorescence of algae (excitation wavelength of $445 \mathrm{~nm}$; emission wavelength of $680 \mathrm{~nm}$ ) was applied to indicate their cell density, since the fluorescence of chlorophyll is in proportion to the density of algae cells $[109,268]$. Prior to the assay, a linear relationship of fluorescence and cell density was observed by using a cell counter (CASY Model TT, Roche, USA). Algae observed in the exponential growing phase were exposed to PhACs samples for 72 hours, based on the method described by Blaise and Ferard [39]. Inhibition of Vibrio fischeri was analysed based on the decay in emitted light when exposed to toxic compounds. The luminescence was recorded after $15 \mathrm{~min}$ exposure at room temperature in darkness. The inhibition was calculated following the established protocol offered by sub-department of Toxicology, Wageningen University, the Netherlands. For the mentioned toxicity assays, $\mathrm{CuSO}_{4}$ as $\mathrm{Cu}$ (II) was selected as the reference compound to validate the protocols (Figure S3.2). Filtrated WWE was set as the blank control.

\subsubsection{Photodegradation kinetics and effect of light attenuation}

When $\mathrm{TiO}_{2}$ was applied as the catalyst, photocatalysis kinetics of organic compounds fit the Langmuir-Hinshelwood model [98], which could be used to describe the reactions between radicals and substrate molecules in either absorbed or dissolved status [205].

$$
-\frac{d C}{d t}=\frac{k_{r} K C}{1+K C}
$$

where $k_{r}$ is the true photodegradation rate of the organic compound $(\mathrm{mg} / \mathrm{l} / \mathrm{h}), C$ the concentration of the compound (mg/l), $t$ the illumination time, and $K$ the adsorption coefficient of the compound to be degraded $(1 / \mathrm{mg}) . k_{r}$ related to several parameters such as mass of catalyst, efficient photon flow, layer of oxygen, etc. [123]. The L-H model can be simplified to a pseudo-first-order kinetic equation and $k$ is the apparent rate of the pseudofirst-order reaction. 
$-\frac{d C}{d t}=k_{r} K C=k C$ or $C=C_{0} e^{-k t}$

In addition, direct photolysis also follows the pseudo-first-order reaction [136]. The photodegration half-life of PhACs could be calculated by

$$
t_{1 / 2}=\frac{\ln 2}{k}
$$

In this study, removal of PhACs by adsorption onto immobilized $\mathrm{TiO}_{2}$ in the dark was insignificant (Figure S3.3), showing that the removal of PhACs in our experiments can be attributed to photodegradation. In surface waters, photodegradation efficiencies are limited by light attenuation by depth [263]. Furthermore, it is reported that the pseudo-first-order kinetic constant of the contaminant is directly proportional to photon fluence underneath the water in direct photolysis $[50,237,393]$. The vertical profiles of down-welling attenuation can be estimated by the Lambert-Beer equation [93]

$$
I_{z}=I_{0} e^{-k_{d} z}
$$

where $I_{0} \quad i$ s the light intensity at water surface measured by the upper sensor (photosynthetically active irradiation, $\mu \mathrm{mol}$ photons $/\left(\mathrm{m}^{2} \mathrm{~s}\right)$ ), $z$ the depth underneath the water surface $(\mathrm{m}), I_{z}$ the light intensity at depth $z$, and $k_{d}$ the light attenuation coefficient $\left(\mathrm{m}^{-1}\right)$. Therefore, for individual compounds, the photodegradation kinetic constant is connected with light intensity as in equation (3.5).

$k=p I_{z}=p I_{0} e^{-k_{d} z}$

where $\mathrm{p}$ is the proportion constant. The value of $k_{d}$ was determined by detecting light intensity at different water depths (Figure S3.4). The slope of $\ln \left(I / I_{0}\right)$ versus water depth gives a $k_{d}$ value of $-3.17 \mathrm{~m}^{-1}$ in DI. 


\subsection{Results and discussion}

\subsubsection{Characteristics of the immobilized catalyst}

Various catalyst support materials have been used to enhance the available surface area of immobilized $\mathrm{TiO}_{2}$ for PhACs removal, including glass spheres [276], glass slides [292], silicon plates [366], titanium alloys [77], fiber sheets [142], and activated carbon [193]. In this work, immobilization of $\mathrm{TiO}_{2}$ on sand was investigated as a potential low-cost and robust catalyst.

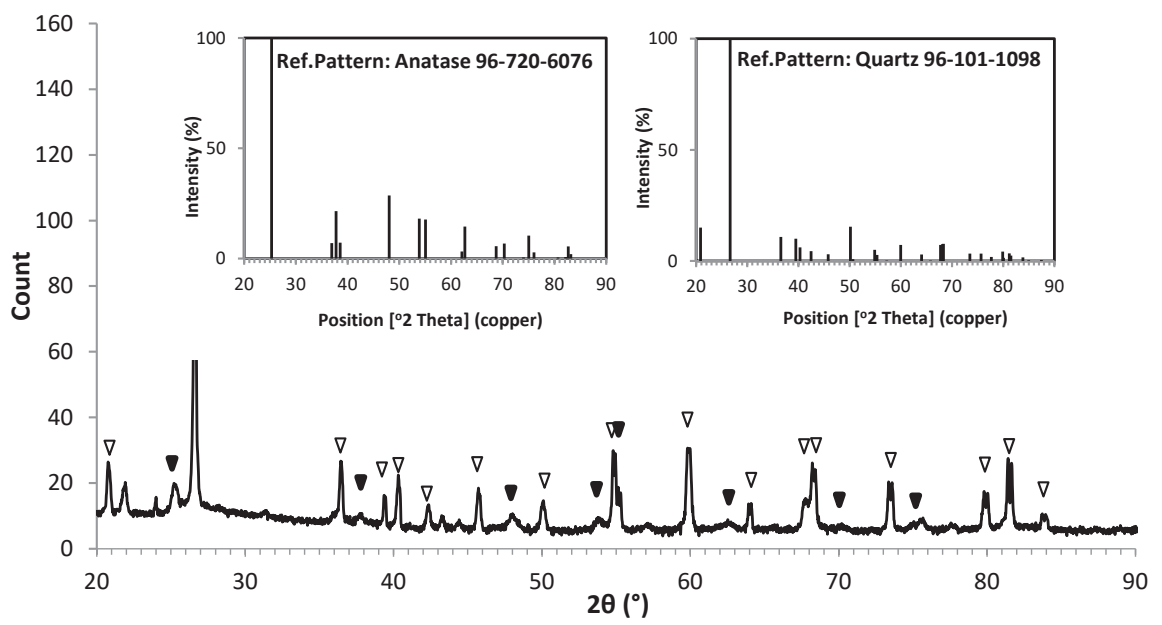

Figure 3.1 XRD pattern of the immobilized $\mathrm{TiO}_{2}$ on sand shows the peaks of anatase phase (close triangle) and quartz phase (open triangle).

The XRD pattern of the immobilized catalyst shows the presence of anatase and quartz phase, and the absence of impurities (Figure 3.1). The characteristic diffraction peaks of $\mathrm{TiO}_{2}$ can be observed at $2 \theta=25.4^{\circ}$ corresponding to the reference plane of anatase, which indicates $\mathrm{TiO}_{2}$ was impregnated in the immobilized material. The anatase phase was produced on purpose during immobilization because this specific phase is preferred over other phases of $\mathrm{TiO}_{2}$ for solar cell application, because anatase exhibits a higher electron mobility, lower dielectric constant, lower density and lower deposition temperature [55]. In addition, the relative intensity of quartz peaks detected was the same as the reference plane, suggesting that the immobilization of $\mathrm{TiO}_{2}$ did not change the structure of the sand. XRD results showed $68.2 \%$ of anatase crystalline phase compared with $31.8 \%$ of quartz phase. The crystallite size of anatase phase $(D, \mathrm{~nm})$ could be estimated by Scherrer's formula $[135,286]$. 
$D=\frac{0.9 \lambda}{\beta \cos \theta}$

where $\lambda$ is the wavelength of X-ray radiation $\left(0.154 \mathrm{~nm}\right.$ for $\mathrm{Cu} K_{\alpha}$ used), $\theta$ the diffraction peak angle, and $\beta$ the difference between the full width at half-maximum of detected sample and the standard sample at certain $\theta$. The crystallite size of anatase was calculated to be 22.6$46.4 \mathrm{~nm}$.

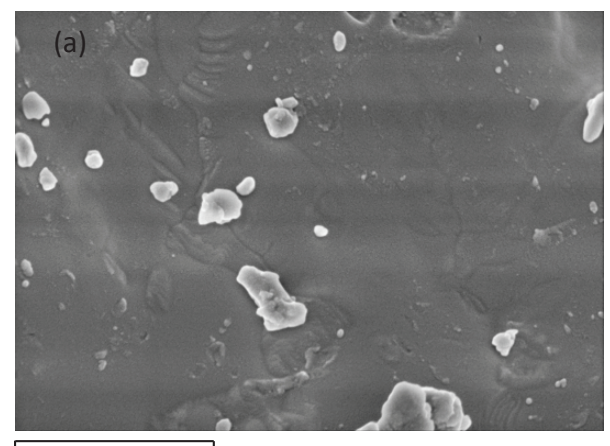

$1 \mu \mathrm{m}$

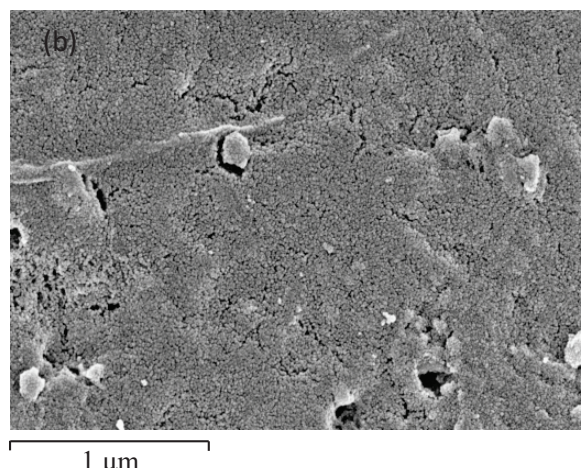

$1 \mu \mathrm{m}$

Figure 3.2 Microstructure of the catalyst (a) surface of raw sand (200-500 $\mu \mathrm{m})$; (b) spherical morphologies of $\mathrm{TiO}_{2}$ grains distributed on sand surface.

SEM images in Figure 3.2 were taken at $100,000 \times$ magnifications to observe the surface morphology of quartz sand before and after immobilization of $\mathrm{TiO}_{2}$. SEM images showed the relatively smooth surface of sand without obvious pores (Figure 3.2a). After immobilization, spherical morphologies of $\mathrm{TiO}_{2}$ grains were observed to be heterogeneously distributed on the sand surface (Figure 3.2b). Microstructure of catalyst did not change after being used twice in the photodegradation experiment. According to the nitrogen gas adsorption isotherms, immobilization of $\mathrm{TiO}_{2}$ increased the specific surface area of raw sand from 0.04 to $2.02 \mathrm{~m}^{2} / \mathrm{g}$. The expected increase was consistence with the morphology showed in SEM. Additionally, the micropore volume of sand increased from almost zero to $0.003 \mathrm{~cm}^{3} / \mathrm{g}$ after immobilization. With the low micropore volume and small pore size (6.88 $\mathrm{nm}$ in diameter), the immobilized catalyst was expected not to adsorb PhACs, which was supported by the results obtained from adsorption tests (Figure S3.3). 


\section{Photodegradation}

\subsubsection{Light attenuation by water: relation between depth and photolysis efficiency}

In order to study the application of solar irradiation in passive photodegradation of PhACs, we investigated the effect of light attenuation by water depth on the photodegradation performance. PhACs samples were collected at different water depths every $24 \mathrm{~h}$. However, counting the detection deviation, there was no obvious concentration difference $(<3 \%)$ between samples at different water depths in $0.5 \mathrm{~m}$ column experiment. Thus, we developed a simplified model to establish the relationship between the pseudo-first-order constant of individual PhACs with water depths. According to the equation (3.2) and (3.5), we calculated $\bar{C}_{t, \text { model }}$, the average concentration of PhACs in the column at certain time points in the model as follows,

$\bar{C}_{t, \bmod e l}=\frac{1}{H} \int_{0}^{H} C_{0} e^{-k t} d z=\frac{1}{H} \int_{0}^{H} C_{0} e^{-p I_{z} t} d z=\frac{1}{H} \int_{0}^{H} C_{0} e^{-p I_{0} e^{-k_{d} z} t} d z$

where $H$ is the total depth of the water column. The experimental value of $\bar{C}_{t, \text { exp }}$ could be obtained by mixing the samples collected at different water depths at certain time points.

In order to yield a more accurate simulation, we simulated and experimentally tested two scenarios: direct photolysis of four PhACs in a water column of $0.1 \mathrm{~m}$ depth in the beaker and $0.5 \mathrm{~m}$ depth in the column. We iterated different values of the proportion constant $p$ to minimize the variance between $\bar{C}_{t, \bmod e l}$ and experimental $\bar{C}_{t, \mathrm{exp}}$. According to the modelling results, $p$ was 0.021 for PRO, 0.040 for $\mathrm{DFC}, 0.010$ for $\mathrm{CBZ}$, and 0.017 for IBP. By integrating the equation (3.5), pseudo-first order constants could be calculated.

$$
k_{\bmod e l}=\frac{1}{H} \int_{0}^{H} p I_{0} e^{-k_{d} z} d z
$$

According to the equation (3.2), the removal efficiencies of PhACs can be calculated using kinetic constants. Then the calculated removal efficiencies were compared with related experimental results (Figure 3.3). 


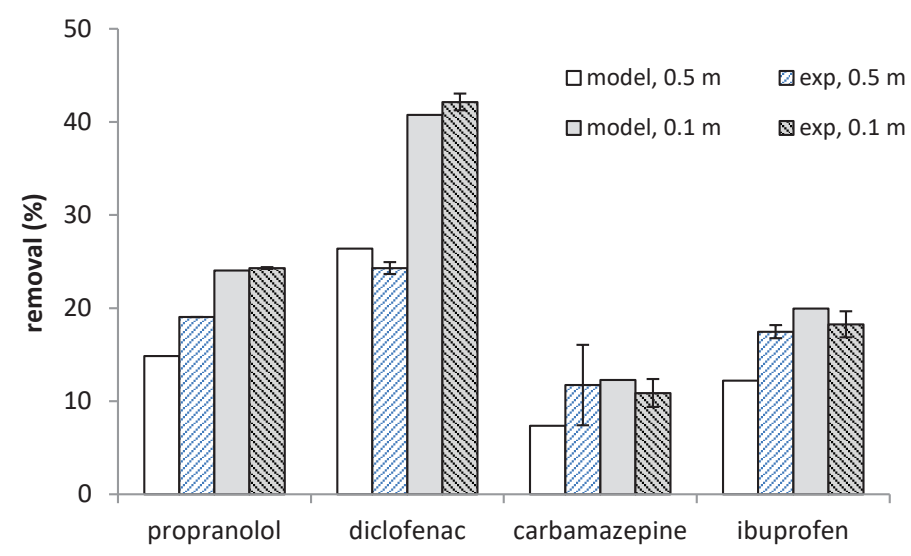

Figure 3.3 Comparison of removal efficiencies of individual PhACs from modelling and experimental set-ups. $96 \mathrm{~h}$ photolysis of $5 \mathrm{mg} / \mathrm{l} \mathrm{PhACs}$ each, spiked in deionized water (DI). Error bars indicate standard errors of the mean (s.e.m.), $n=2$.

In Figure 3.3, the modelling results indicate high similarity in removal efficiencies of four PhACs compared to the efficiencies obtained from experiments performed at water depth of $0.5 \mathrm{~m}$ and $0.1 \mathrm{~m}$, respectively. Additionally, according to the regression analysis in Figure S3.5, the modelling concentrations of four PhACs are approximate to the related experimental values. Thus, the model describes the experimental results adequately, indicating the proposed attenuation mechanisms to be active. Furthermore, we could predict the relationship between water depth and direct photolysis removal efficiency of individual PhACs (Figure 3.4). The data presented here did not account for the water quality containing various components and other site-specific factors, but only focused on the direct photolysis of PhACs. Results suggest that removal of PhACs by direct photolysis distinctly decreased with water depth when the depth is less than $1.0 \mathrm{~m}$, while the removal efficiencies are insignificant when the water column is deeper than $1.0 \mathrm{~m}$ (Figure 3.4). However, for the natural attenuation of PhACs in waters, as calculated by our work, even the water depth was close to 0 , the removal efficiencies were still not satisfying, with approximately $26 \%$ for PRO, $43 \%$ for DFC, $13 \%$ for CBZ, and $21 \%$ for IBP. Therefore, it is essential to apply a catalyst to improve the photodegradation performance. For the following kinetic study, we completed the experiment by applying a water depth of $0.1 \mathrm{~m}$ to achieve higher removal performance based on our experimental set-up. 


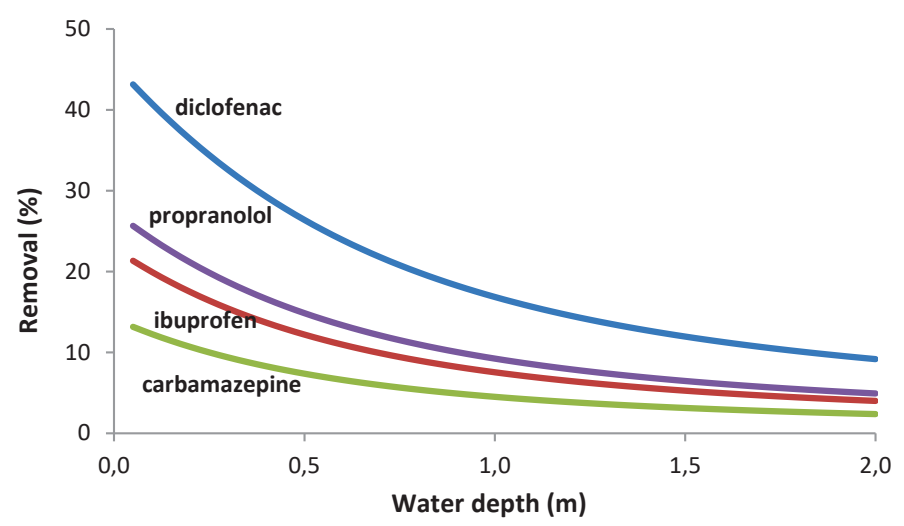

Figure 3.4 Relationship of PhACs removal versus water depth obtained from validated modelling, 96 $\mathrm{h}$ direct photolysis of PhACs spiked in deionized water (DI). Relatively higher removal efficiency corresponded to a depth less than $1.0 \mathrm{~m}$.

\subsubsection{Photodegradation performance, kinetics and mechanisms}

Following the optimized water depth, we evaluated the effect of immobilized $\mathrm{TiO}_{2}$ on $\mathrm{PhACs}$ removal in a simplified system, which is performing photolysis and photocatalysis in DI for $96 \mathrm{~h}$. With respect to the PhACs spiked in DI, both photolysis and photocatalysis of PhACs fit the pseudo-first-order kinetics and the slope represents the kinetic constant (Figure S3.6). However, photocatalysis showed notably better performance in eliminating PhACs than photolysis (Figure 3.5). The half-life time of PRO and DFC under simulated solar irradiation were decreased from 239 and $121 \mathrm{~h}$ to 13 and $12 \mathrm{~h}$ due to addition of the catalyst (Table 3.1). The less photo-active PhACs, CBZ and IBP showed long half-lift times (578 h for CBZ; 330 $\mathrm{h}$ for IBP) under photolysis (Table 3.1), which is comparable to a study by Matamoros et al. [263], in which CBZ and IBP were exposed to solar light in May in Barcelona. However, for photocatalysis, the half-life time of CBZ and IBP were only 25 and $21 \mathrm{~h}$, indicating a good performance of the catalyst, which was also confirmed by the formation of by-products detected by UPLC. Chromatography results showed more by-products formed and further degraded in photocatalysis than in photolysis (Figure S3.7), indicating the process of photocatalysis was much faster. Thus, by producing more active hydroxyl radicals $\left({ }^{\circ} \mathrm{OH}\right)$, the immobilized catalyst showed significant enhancement for removing PhACs. 

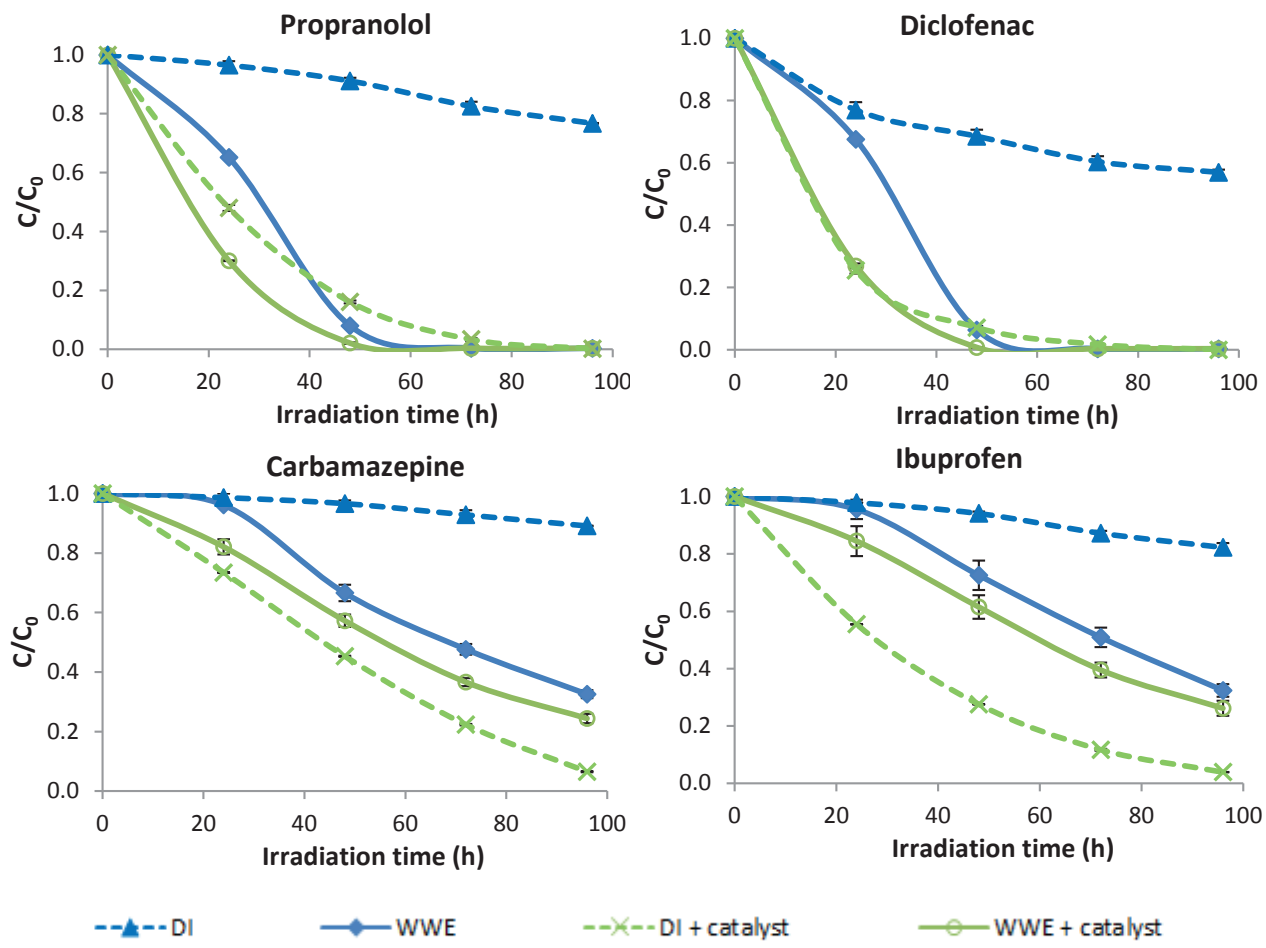

$--\ngtr--\mathrm{DI}+$ catalyst

WWE + catalyst

Figure 3.5 Photolysis and photocatalyis of PhACs versus irradiation time. $5 \mathrm{mg} / 1$ for each $\mathrm{PhAC}$ spiked in deionized water (DI) and wastewater effluent (WWE) under $96 \mathrm{~h}$ simulated solar irradiation. Data points and error bars are average values/standard errors of the mean (s.e.m.), $n=2$.

To understand and determine the effect of immobilized $\mathrm{TiO}_{2}$ in a real water matrix, the catalyst was applied in the WWE. In WWE, photocatalysis of PhACs also performed better than photolysis (Figure 3.5). Although catalyst addition yielded a smaller improvement in removal efficiencies in WWE when compared with the DI photodegradation, the removal efficiencies of PRO, DFC, CBZ, and IBP were 100\%, 100\%, 76\%, and 74\% respectively after $96 \mathrm{~h}$ photocatalysis (Table 3.1). Especially for the PhACs recalcitrant towards biodegradation, PRO, DFC and CBZ, photocatalysis under simulated solar light showed considerable removal performance. Additionally, the formation and depletion of by-products in photocatalysis was faster compared with photolysis (Figure 3.6). For example, the byproduct at $10.03 \mathrm{~min}$ in the chromatogram was produced within $24 \mathrm{~h}$ irradiation and depleted after $72 \mathrm{~h}$ in photocatalysis, while it only appeared and accumulated after $48 \mathrm{~h}$ irradiation in photolysis. 


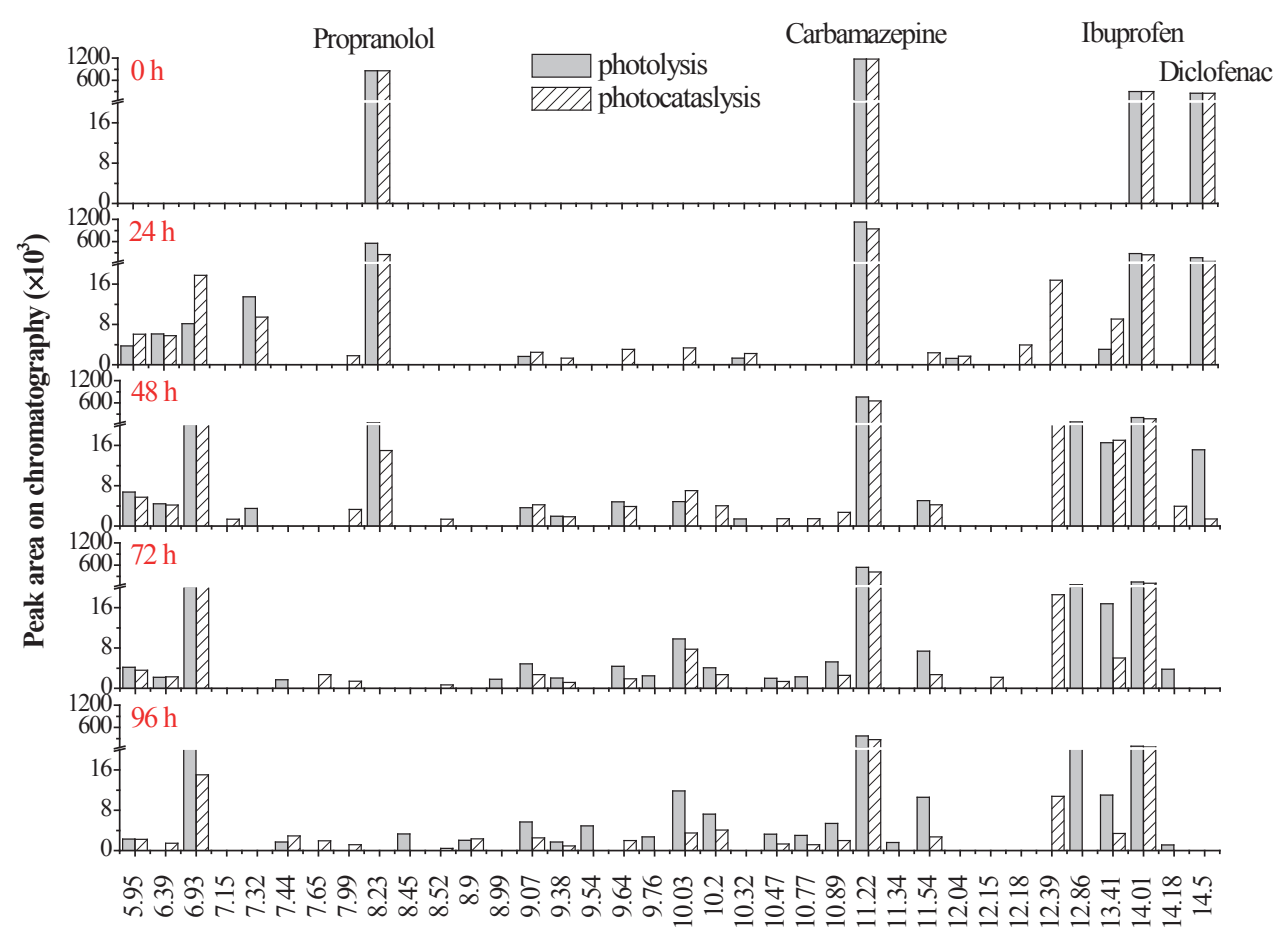

Retention time on chromatography (min)

Figure 3.6 Presence and variation of by-products at different irradiation time points showed in chromatography spectrum. Photolysis and photocatalysis of PhACs spiked in wastewater effluent (WWE).

Photolysis constants of PhACs in WWE were upwards of ten times higher compared with the related values in DI. For PRO and DFC in DI, the high constants were even comparable with the constants obtained when the catalyst was present (Table 3.1). This is possibly caused by the enhancement of degradation by natural dissolved organic matter (DOM) in WWE $(\mathrm{DOM}=12.2 \mathrm{mg} \mathrm{C} / \mathrm{l})$, which could absorb light to excite triplet states of DOM ( $\left.{ }^{3} \mathrm{DOM}^{*}\right)$ and further to ${ }^{\circ} \mathrm{OH}$. ${ }^{\circ} \mathrm{OH}$ could subsequently oxidize PhACs into by-products or $\mathrm{CO}_{2}$, shown as the mechanism 4 in Figure 3.7 and further elaborated below. ${ }^{3} \mathrm{DOM}^{*}$ could enhance the transformation of aquatic contaminants by acting as a photosensitizer [51], especially for aromatic pollutants with a phenolic OH group [364], such as PRO in our case (Table S2.1). Chen et al. [67] verified fulvic acid, a generic form of DOM, to be the main photochemically reactive species to interact with PRO. The kinetic constant of PRO increased from $0.069 \mathrm{~h}^{-1}$ to $0.230 \mathrm{~h}^{-1}$ after adding fulvic acid $(5 \mathrm{mg} \mathrm{C} / 1)$. In addition, the first order constant of DFC 
proved to increase twice higher under UV irradiation after adding effluent organic matter of $10.2 \mathrm{mg}$ C/1 from a WWTP in Korea [216].

Table 3.1 Pseudo-first-order kinetic constants and removal performance of PhACs under $96 \mathrm{~h}$ photolysis and photocatalysis. $5 \mathrm{mg} / \mathrm{l}$ for each PhAC spiked in deionized water (DI) and wastewater effluent (WWE).

\begin{tabular}{|c|c|c|c|c|c|c|c|c|c|}
\hline \multirow[b]{2}{*}{ Matrix } & \multirow[b]{2}{*}{ PhACs } & \multicolumn{4}{|c|}{ Photolysis } & \multicolumn{4}{|c|}{ Photocatalysis } \\
\hline & & $\begin{array}{l}\text { Removal } \\
(\%)\end{array}$ & $\begin{array}{l}\mathrm{k}_{\mathrm{con}} \\
\left(\mathrm{h}^{-1}\right)\end{array}$ & $\mathrm{r}^{2}$ & $\mathrm{t}_{1 / 2}(\mathrm{~h})$ & $\begin{array}{l}\text { Removal } \\
(\%)\end{array}$ & $\mathrm{k}_{\mathrm{con}}\left(\mathrm{h}^{-1}\right)$ & $r^{2}$ & $\begin{array}{l}\mathrm{t}_{1 / 2} \\
\text { (h) }\end{array}$ \\
\hline \multirow{4}{*}{ DI } & PRO & $23.3 \pm 0.1$ & -0.0029 & 0.98 & 239.0 & $99.8 \pm 0.2$ & $-0.0523 \pm 0.0088$ & 0.96 & 13.3 \\
\hline & DFC & $43.3 \pm 0.9$ & $-0.0057 \pm 0.0001$ & 0.92 & 121.6 & 100 & $-0.0562 \pm 0.0022$ & 1.00 & 12.3 \\
\hline & $\mathrm{CBZ}$ & $10.9 \pm 1.5$ & $-0.0012 \pm 0.0001$ & 0.97 & 577.6 & $93.5 \pm 0.1$ & $-0.0277 \pm 0.0002$ & 0.93 & 25.0 \\
\hline & IBP & $17.7 \pm 1.4$ & $-0.0021 \pm 0.0001$ & 0.96 & 330.1 & $96.1 \pm 0.2$ & $-0.0336 \pm 0.0003$ & 0.98 & 20.7 \\
\hline \multirow{4}{*}{ WWE } & PRO & 100 & $-0.0527 \pm 0.0003$ & 0.87 & 13.2 & 100 & $-0.0808 \pm 0.0027$ & 0.95 & 8.6 \\
\hline & DFC & 100 & $-0.0573 \pm 0.0005$ & 0.85 & 12.1 & 100 & $-0.1018 \pm 0.0033$ & 0.93 & 6.8 \\
\hline & $\mathrm{CBZ}$ & $67.4 \pm 2.2$ & $-0.0122 \pm 0.0007$ & 0.96 & 57.0 & $75.6 \pm 2.6$ & $-0.0150 \pm 0.0008$ & 0.98 & 46.2 \\
\hline & IBP & $67.6 \pm 1.4$ & $-0.0119 \pm 0.0005$ & 0.94 & 58.2 & $73.8 \pm 1.6$ & $-0.0142 \pm 0.0006$ & 0.98 & 48.8 \\
\hline
\end{tabular}

Nitrate could adsorb solar light when $\lambda<350 \mathrm{~nm}[246]$ and produce ${ }^{\circ} \mathrm{OH}$ to accelerate the indirect photodegradation of PhACs. Compared with DOM, the light screening effect of nitrate is negligible with its very low molar extinction coefficient [68]. Ji et al. [186] reported an enhanced photodegradation rate constant of atenolol from $0.001 \mathrm{~min}^{-1}$ to $0.007 \mathrm{~min}^{-1}$, when the concentration of nitrate increased from $0.5 \mathrm{mM} / 1$ to $10 \mathrm{mM}$. In this study, the concentration of WWE was $0.07 \mathrm{mM} / 1$ (Table S3.2), much lower than the level in previous literature studying the effect of nitrate on micropollutants removal. Thus, the presence of nitrate might insignificantly contribute to enhancing photodegradation of PhACs.

On the other hand, DOM and other inorganic ions including $\mathrm{CO}_{3}{ }^{2-}$ and $\mathrm{HCO}_{3}{ }^{-}$could inhibit the photodegradation. DOM is the main inhibitor while inorganic ions contribute insignificant to scavenging [382]. There are three main mechanisms by which DOM can inhibit photodegradation, that is 1 ) DOM acts as a quencher of reactive species such as ${ }^{\circ} \mathrm{OH} ; 2$ ) DOM screens photo-chemically active light; or 3) DOM reacts with contaminant intermediates (Figure 3.7). 


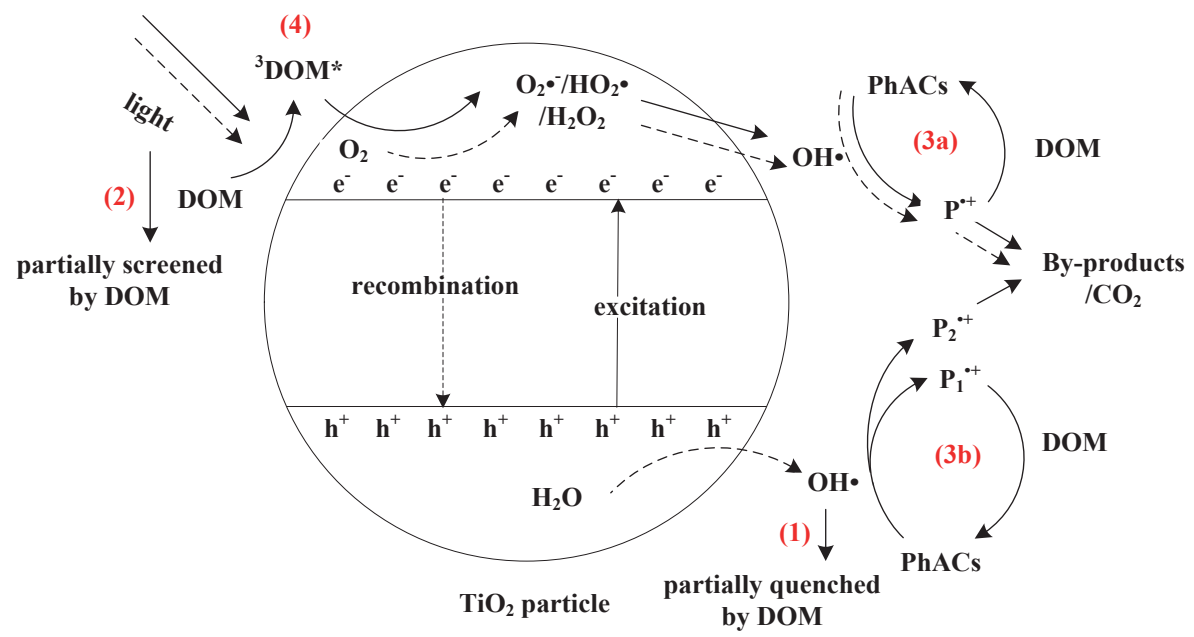

Figure 3.7 Hypothetical mechanisms of photocatalysis of PhACs (P) without DOM (dash lines), and the role of DOM as the photosensitizer and inhibitor (solid lines). The inhibition of DOM could be due to (1) quenching $\bullet \mathrm{OH}$; (2) screening active photons; (3) reacting with oxidized $\mathrm{PhACs}\left(\mathrm{P}^{{ }^{++}}\right.$or $\mathrm{P}_{1}{ }^{\cdot+}$ ). The enhancement of DOM could be due to (4) production of ${ }^{3} \mathrm{DOM}^{*}$ and further to ${ }^{\circ} \mathrm{OH}$.

For the first proposed mechanism, in our case with $12.2 \mathrm{mg} \mathrm{C} / 1 \mathrm{DOM}$, the first mechanism might be negligible, because no significant ${ }^{\circ} \mathrm{OH}$ quenching effect $(>10 \%)$ on aromatic ketones was detected when the concentration of DOC was below 22-72 mg/1 [374].

As discussed in section 2.5, photodegradation of PhACs in this study followed pseudo-first order kinetics, which means an exponential decrease of the concentration of PhACs in time. However, in the case of photolysis in WWE, completed exponential tendencies were not detected for all the PhACs due to an apparent delay phase observed in the first $24 \mathrm{~h}$ (Figure 3.5). This might be caused by the light screening of DOM present in WWE (Figure 3.7), which is the second proposed mechanism. DOM has a coloured or chromophore fraction which could absorb solar light and modify the spectrum of radiation penetrating into the water column [215]. Similarly, the same phenomenon was also observed by Dong et al. [99], in which the photodegradation of atenolol, carbamazepine, and primidone in WWE was delayed in the first 24 or $48 \mathrm{~h}$ under solar irradiation. After the delay phase, production of $\mathrm{OH}$ appeared to be dominant and photodegradation proceeded at an increasing rate. The delay phase was much shorter or absent in case photocatalysis was applied, and this is consistent with the higher amounts of radicals produced by the catalyst. 
In the third proposed mechanism, after reacting with ${ }^{\bullet} \mathrm{OH}$ or ${ }^{3} \mathrm{DOM}^{*}, \mathrm{PhACs}$ transform to a radical cation, $\mathrm{P}^{\cdot+}$ (in the case of transferring one electron) or to different types of intermediate radical cations such as $\mathrm{P}_{1}{ }^{++}$and $\mathrm{P}_{2}{ }^{-+}$, shown as path $3 \mathrm{a}$ and $3 \mathrm{~b}$ in Figure 3.7. A portion of $\mathrm{P}^{\cdot+}$ could either be further irreversibly transformed to by-products or to $\mathrm{CO}_{2}$ (as in $\mathrm{P}_{1}{ }^{++}$), or be reduced by DOM to reform the parent compounds (as in $\mathrm{P}_{2}{ }^{++}$) [50,373]. DOM contains a variety of organic moieties, especially the phenolic functional groups which could act as antioxidants to reduce the oxidation intermediates $\mathrm{P}^{\bullet+}[373]$. This process was shown by investigating the photo-depletion of 22 contaminants in a photoreactor installed with a mercury lamp [51]. They found that the depletion rate of half of the target contaminants decreased in the presence of Suwannee River fulvic acid which was used as their reference DOM.

In WWE, when more ${ }^{\circ} \mathrm{OH}$ were produced by the catalyst, the first-order constants of PRO and DFC increased, while the constants of CBZ and IBP were almost the same with those in photolysis (Table 3.1). However, when the catalyst was present, CBZ and IBP have the potential to be further degraded, as the removal efficiencies of those two compounds yielded higher level in DI compared with WWE. As the inhibition of DOM through mechanism 1 and 2 are identical for all the PhACs in mixture, the different levels of inhibition between different PhACs were possibly caused by mechanism 3. It is reported that DOM mediated indirect photolysis of organic compounds is partly driven by the chemical reactivity of the contaminants [147]. In our work, during the competition of ${ }^{\circ} \mathrm{OH}$ with the other $\mathrm{PhACs}$ in DI photocatalysis experiment, CBZ and IBP are more recalcitrant to degradation. Thus, intermediate radical cations of CBZ and IBP $\left(\mathrm{P}^{*+}\right)$ could be more easily reduced back to their parent compounds. We hypothesize that the intermediate radical cations of CBZ and IBP have been selectively reformed to parent compounds through reduction by DOM, which is in line with mechanism $3 \mathrm{~b}$. That means no matter how much $\mathrm{P}_{1}{ }^{++}$is produced by reactive species, $\mathrm{P}_{1}{ }^{++}$will be reduced to $\mathrm{P}$ by sufficient DOM while the production of irreversible byproducts could only depend on the production of $\mathrm{P}_{2}{ }^{\cdot+}$, resulting in no increase of kinetic constants of CBZ and IBP even though the catalyst provides more reactive species. However, for PRO and DFC, we could not conclude whether DOM inhibited their depletion rates because their kinetic constants in WWE photocatalysis were higher compared with the constants in DI photocatalysis. Assuming similar DOM inhibition for PRO and DFC, the story might be different from that of $\mathrm{CBZ}$ and IBP. When more $\mathrm{P}^{{ }^{+}}$is produced due to catalysis, even though parts of the $\mathrm{P}^{\cdot+}$ were transformed to parent PRO and IBP, there were 


\section{Photodegradation}

still more by-products formed because of the excessive $\mathrm{P}^{\cdot+}$ (Figure 3.7). This conforms to mechanism 3a. Wenk et al. [373] also showed that the theory of $\mathrm{P}^{\cdot+}$ fits for sulfamethoxazole while the theory of $\mathrm{P}_{1}{ }^{++}$and $\mathrm{P}_{2}{ }^{\cdot+}$ is suitable for $\mathrm{N}, \mathrm{N}$-dimethylaniline and trimethoprim, when $5 \mu \mathrm{M}$ of the components are exposed to a mercury lamp with DOM in 0.1-2.9 mg C/1.

It is reported that the DOM type in WWE greatly varies among different water treatment systems, hence unexpected inhibitions of oxidation might occur [41]. Alternatively natural waters and wetland DOM might enhance photodegradation of PhACs otherwise conservative in degradation [216]. In order to understand the relationship between the DOM structure and its potential role in photodegradation, we performed FTIR analysis of the WWE solid. From the FTIR spectra in Figure S3.8, we found absorption bands that correspond to the following functional groups [21, 347]: 1) $3352 \mathrm{~cm}^{-1}(\mathrm{O}-\mathrm{H}$ stretching of inter- and intra-molecular hydrogens bonds); 2) $1638 \mathrm{~cm}^{-1}$ (aromatic $\mathrm{C}=\mathrm{C}$ skeletal vibrations, asymmetric stretching of $\mathrm{C}=\mathrm{O}$ of quinones and ketones, symmetric stretching of $\mathrm{COO}^{-}$); 3) $1406 \mathrm{~cm}^{-1}$ (asymmetric stretching of $\mathrm{COO}^{-}, \mathrm{C}-\mathrm{H}$ bending of aliphatic groups); 4) $1188 \mathrm{~cm}^{-1}(\mathrm{C}-\mathrm{O}$ asymmetric stretching and $\mathrm{OH}$ bending of $\mathrm{COOH}$ groups and phenols); 5) $1101 \mathrm{~cm}^{-1}(\mathrm{C}-\mathrm{O}$ stretching of primary alcohols and in-plane bending of aromatic $\mathrm{C}-\mathrm{H}$. These results show the presence of aromatic and phenolic functional groups, and this might explain the complicated role of DOM in photodegradation not only as a photosensitizer, but also as an inhibitor for PhACs photodegradation. DOM could produce ${ }^{3} \mathrm{DOM}^{*}$ under irradiation, which could enhance the transformation of aquatic contaminants by acting as a photosensitizer [51]. However, it is also reported that highly aromatic DOM generally presents a higher efficiency in inhibiting ${ }^{3} \mathrm{DOM}^{*}$-induced oxidation and a lower photochemical reactivity. Furthermore, phenolic functional groups could act as antioxidants to reduce the oxidation intermediates $\mathrm{P}^{\mathbf{p}^{+}}[373]$.

\subsubsection{Toxicity and biodegradability}

In order to evaluate the feasibility and environmental impact of the photodegradation for treating WWE spiked with PhACs, toxicity tests were performed in parallel with sampling for PhACs. It is reported that non-stable by-products formed during photodegradation may pose environmental risks [2]. Therefore, the toxicity of PhACs during photodegradation is essential when assessing the feasibility of water treatment techniques. 


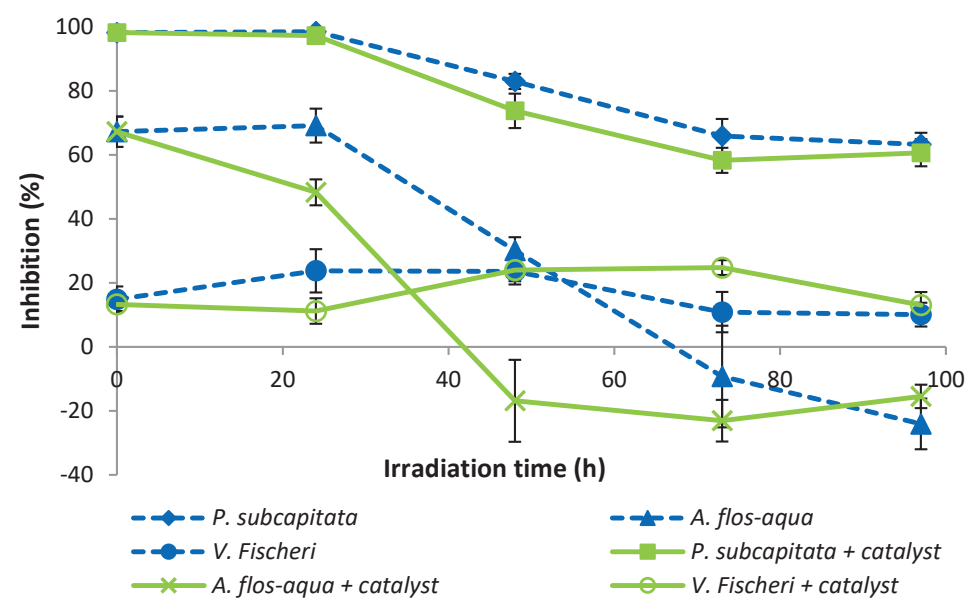

Figure 3.8 Toxicity of PhACs spiked in wastewater effluent (WWE) during photocatalysis and photolysis. $5 \mathrm{mg} / 1$ for each $\mathrm{PhAC}$ under $96 \mathrm{~h}$ simulated solar irradiation. Green algae (Pseudokirchneriella subcapitata) and blue-green algae (Anabaena flos-aqua) exposed to PhACs for $72 \mathrm{~h}$ in algae incubator; luminescence bacteria (Vibrio fischeri) exposed for $15 \mathrm{~min}$. Data points and error bars are average values/standard deviation, $n=5$.

We used known aquatic microorganisms, including green algae, blue-green algae, and luminescence bacteria, to test the chronic ( $72 \mathrm{~h}$ exposure for algae species) and acute (15 min exposure for bacteria) toxicity of PhACs spiked in WWE. Previous studies mainly focus on the toxicity of parent compounds [45], synergistic toxicity of PhACs and related by-products before and after photodegradation [95], or toxicity of PhACs during photodegradation in DI rather than the real water matrix [308].

The inhibition of microorganisms was calculated by setting WWE without spiking PhACs as the blank control. In Figure 3.8, the presence of PhACs indeed inhibited the target microorganisms, among which the green algae seemed to be more sensitive to the PhACs with almost $100 \%$ growth inhibition before photodegradation. During $96 \mathrm{~h}$ irradiation, chronic toxicity of PhACs to the algal species decreased. Similarly, in the work of Rizzo et al. [307], the toxicity of mixed PhACs (10 mg/l amoxicillin, $5 \mathrm{mg} / \mathrm{l} \mathrm{CBZ,} 2.5 \mathrm{mg} / \mathrm{l} \mathrm{DFC}$ ) to $P$. subcapitata gradually decreased from $96 \%$ to $60 \%$ inhibition when the PhACs were degraded by UV irradiation for $2 \mathrm{~h}$. For the blue-green algae, a dramatic decrease in toxicity was achieved yielding negative inhibition, i.e. a slight stimulation. This fact might be because residual $\mathrm{CBZ}$, IBP, and the photodegradation by-products (Figure 3.6) are mildly toxic or 


\section{Photodegradation}

non-toxic, while the high content of organic matter in WWE is available for the cells as a nutrition resource resulting in growth. This enhanced growth of blue-green algae was also found in the control wells filled with WWE only. For the luminescence bacteria, the produced intermediates seemed to be similarly toxic than the parent compounds during the photodegradation, followed by a slight decreased tendency.

Overall, the algal growth inhibition assays suggested that photocatalysis promoted the formation of less toxic by-products and the toxicity decreased with prolonging treatment time. Additionally, photocatalysis caused a more obvious reduction of toxicity compared with photolysis. On the basis of the results, we could conclude that the photocatalysis technique not only yielded high PhACs removal efficiencies, but also led to a reduction in the toxicity after treatment.

Table 3.2 Biodegradability of wastewater effluent (WWE) before and after being treated by photolysis and photocatalysis.

\begin{tabular}{llll}
\hline Conditions & $\mathrm{BOD}_{5}(\mathrm{mg} / \mathrm{l})$ & $\mathrm{COD}(\mathrm{mg} / \mathrm{l})$ & $\mathrm{BOD}_{5} / \mathrm{COD}$ \\
\hline WWE & $6.0 \pm 1.0$ & $35.2 \pm 0.6$ & 0.17 \\
WWE spiked with mixed PhACs & $12.7 \pm 0.3$ & $67.0 \pm 2.8$ & 0.19 \\
After photolysis treatment of spiked WWE & $12.7 \pm 0.1$ & $61.9 \pm 1.6$ & 0.19 \\
After photocatalysis treatment of spiked WWE & $13.7 \pm 2.7$ & $46.6 \pm 3.0$ & 0.29 \\
\hline
\end{tabular}

In practical cases, PhACs could be further degraded downstreams in natural water systems, such as natural wetlands, ponds or lagoons before discharge to lakes and rivers [80]. In order to evaluate the suitability of the photocatalytic treatment in wastewater containing PhACs, biodegradability $\left(\mathrm{BOD}_{5} / \mathrm{COD}\right)$ was assessed before and after photolysis and photocatalysis. After spiking $5 \mathrm{mg} / \mathrm{L}$ PhACs of each, COD of WWE increased by $31.8 \mathrm{mg} / \mathrm{l}$ while $\mathrm{BOD}_{5}$ increased by $6.7 \mathrm{mg} / \mathrm{l}$ (Table 3.2), suggesting that only a small fraction of PhACs have the potential to be metabolized by bacteria within test period of $\mathrm{BOD}_{5}$. Moreover, the $\mathrm{BOD}_{5} / \mathrm{COD}$ ratio was lower than 0.20 , which means the WWE containing PhACs was hardly biodegradable [82], thus preventing the direct application of bio-treatment techniques. After photocatalysis, $\mathrm{COD}$ decreased due to the mineralization of PhACs into by-products or $\mathrm{CO}_{2}$. Comparison of COD levels after photolysis and photocatalysis indicates that photocatalysis removed organic matter more efficiently. However, $\mathrm{BOD}_{5}$ did not show an obvious increase 
following either treatment. Even though some by-products were more completely degraded during the photocatalysis, 18 different by-products were detected at the end (Figure 3.6). These residual by-products were not easily photo-degradable under the experimental conditions in our work. Overall, the decrease of toxicity and slight increase of biodegradability showed that photocatalysis has the potential to improve the susceptibility of effluent to further biodegradation in natural surface water systems such as wetlands and lagoons.

\subsection{Conclusions}

The photolysis and photocatalysis of four selected PhACs spiked in WWE, has been demonstrated under simulated solar irradiation. The photodegradation pattern followed pseudo-first order kinetics. By using the immobilized $\mathrm{TiO}_{2}$ on sand as the catalyst, removal efficiencies of PhACs were significantly improved in DI. In the case of WWE, the removal rates of PRO and DFC were higher in photocatalysis than in photolysis; the rates of CBZ and IBP did not obviously increase. However, the degradation of PhACs to form by-products and further degradation of these by-products were faster in photocatalysis compared with photolysis. DOM could enhance the photodegradation efficiency by producing more reactive species, while it could also inhibit the degradation performance. A possible mechanism contributing to this is a partial or selective reduction of the oxidized PhACs back to parent compounds. After photocatalysis, the chronic toxicity of PhACs decreased for the aquatic microorganisms, green algae and blue-green algae. In addition, the biodegradability of WWE spiked with PhACs was slightly improved.

From a perspective of application, the photocatalysis was performed by combining the economical and robust components including $\mathrm{TiO}_{2}$, sand, and solar light. Additionally, photocatalysis under simulated solar light showed considerable removal performance for the PhACs recalcitrant towards biodegradation, PRO, DFC and CBZ. Therefore, applying the photocatalysis in an open shallow treatment cell as a post-treatment technique would be beneficial for the attenuation of PhACs in the downstream located natural water systems, such as wetlands, lagoons, or surface waters. 


\section{Acknowledgement}

Authors thank Harry Bruning for helpful modelling discussion, Tinka Murk and Ziqiu Su for the assistance of toxicity assays. The support provided by China Scholarship Council (CSC) during a study of Yujie He in Wageningen University is acknowledged. 


\section{Supplementary materials}

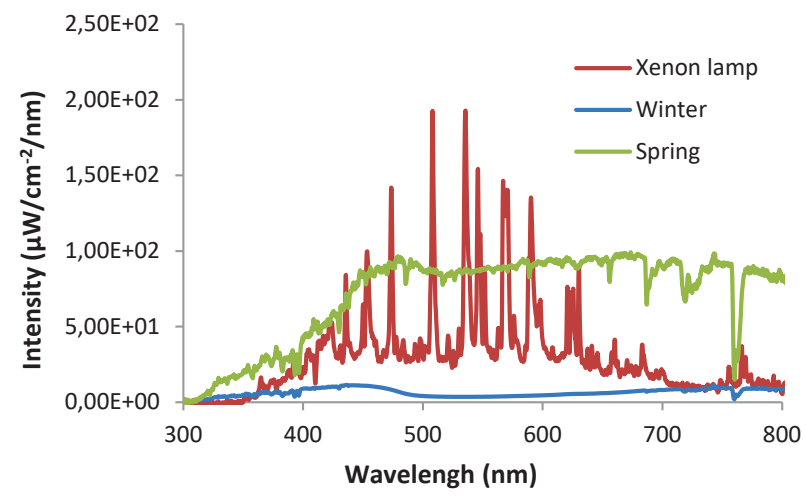

Figure S3.1 Spectrum of sunlight (latitude 51 $59^{\prime} 4^{\prime}$ 'N, longitude 5'39'31'’E, December and March) and Xe-lamp.

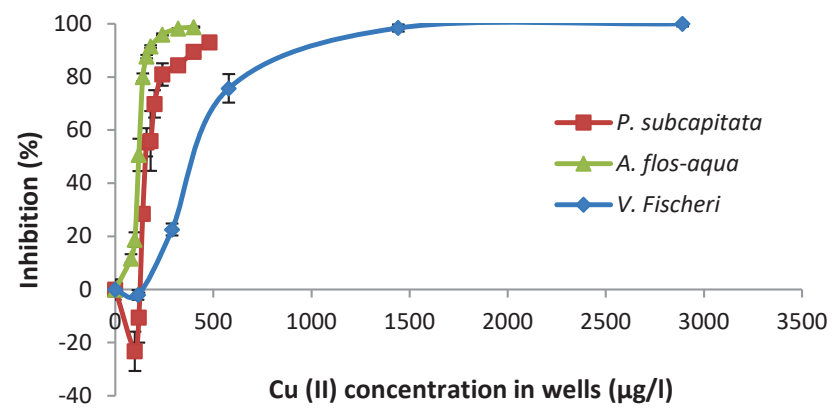

Figure S3.2 Toxicity of $\mathrm{Cu}$ (II) as a reference compound to microorganisms. Green algae (Pseudokirchneriella subcapitata) and blue-green algae (Anabaena flos-aqua) exposed to $\mathrm{Cu}$ (II) for $72 \mathrm{~h}$ in algae incubator; luminescence bacteria (Vibrio fischeri) exposed for $15 \mathrm{~min}$. Data points and error bars are average values/standard deviation, $n=5$. 


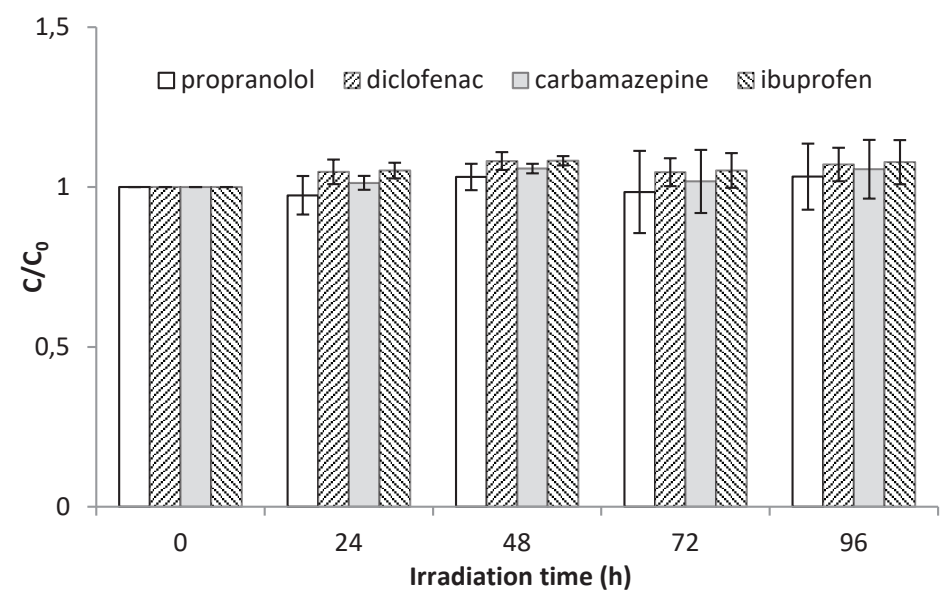

Figure S3.3 Concentration of PhACs under $96 \mathrm{~h}$ adsorption, showing almost no adsorption of PhACs onto immobilized catalyst. $5 \mathrm{mg} / \mathrm{l}$ for each PhAC spiked in wastewater effluent. Data points and error bars are average values/standard errors of the mean (s.e.m.), $n=2$.

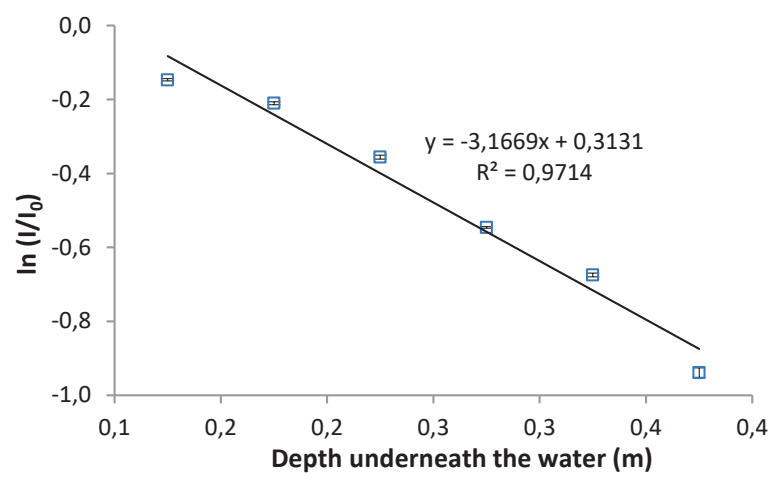

Figure S3.4 Linearity relationship between light intensity versus water depth. Data points and error bars are average values/standard deviation, $n=10$. 


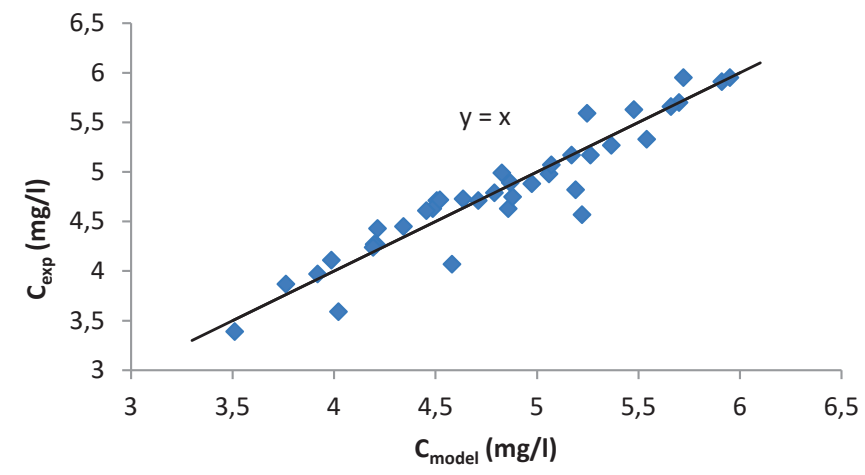

Figure S3.5 Regression analysis between modelling concentrations of four PhACs and related experimental concentrations.
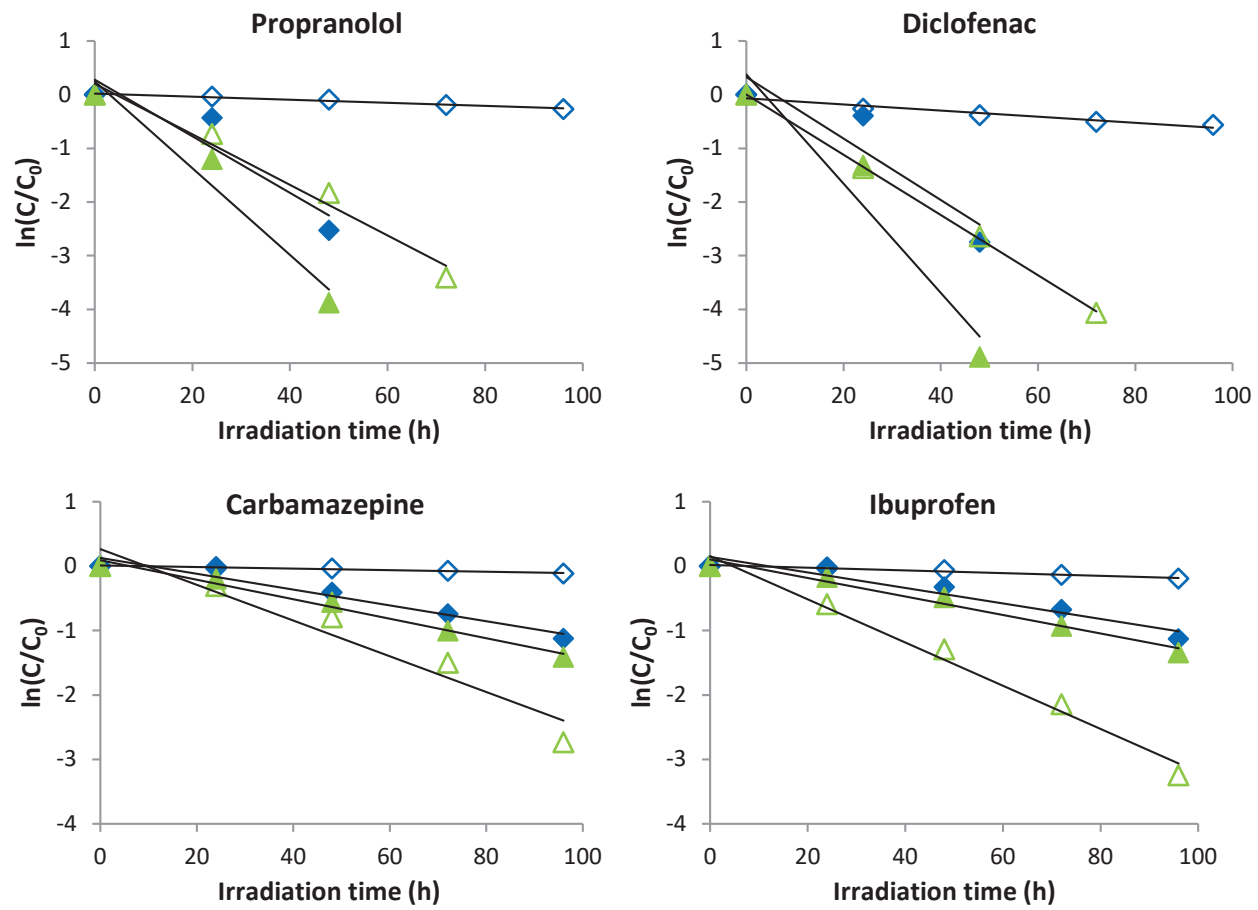

$\diamond \mathrm{DI}$

- WWE

$\triangle \mathrm{DI}+$ catalyst

$\triangle$ WWE + catalyst

Figure S3.6 Photolysis and photocatalysis of PhACs followed pseudo-first-order kinetics, $5 \mathrm{mg} / 1$ for each PhAC spiked in deionized water (DI) and wastewater effluent (WWE) under $96 \mathrm{~h}$ simulated solar irradiation. 


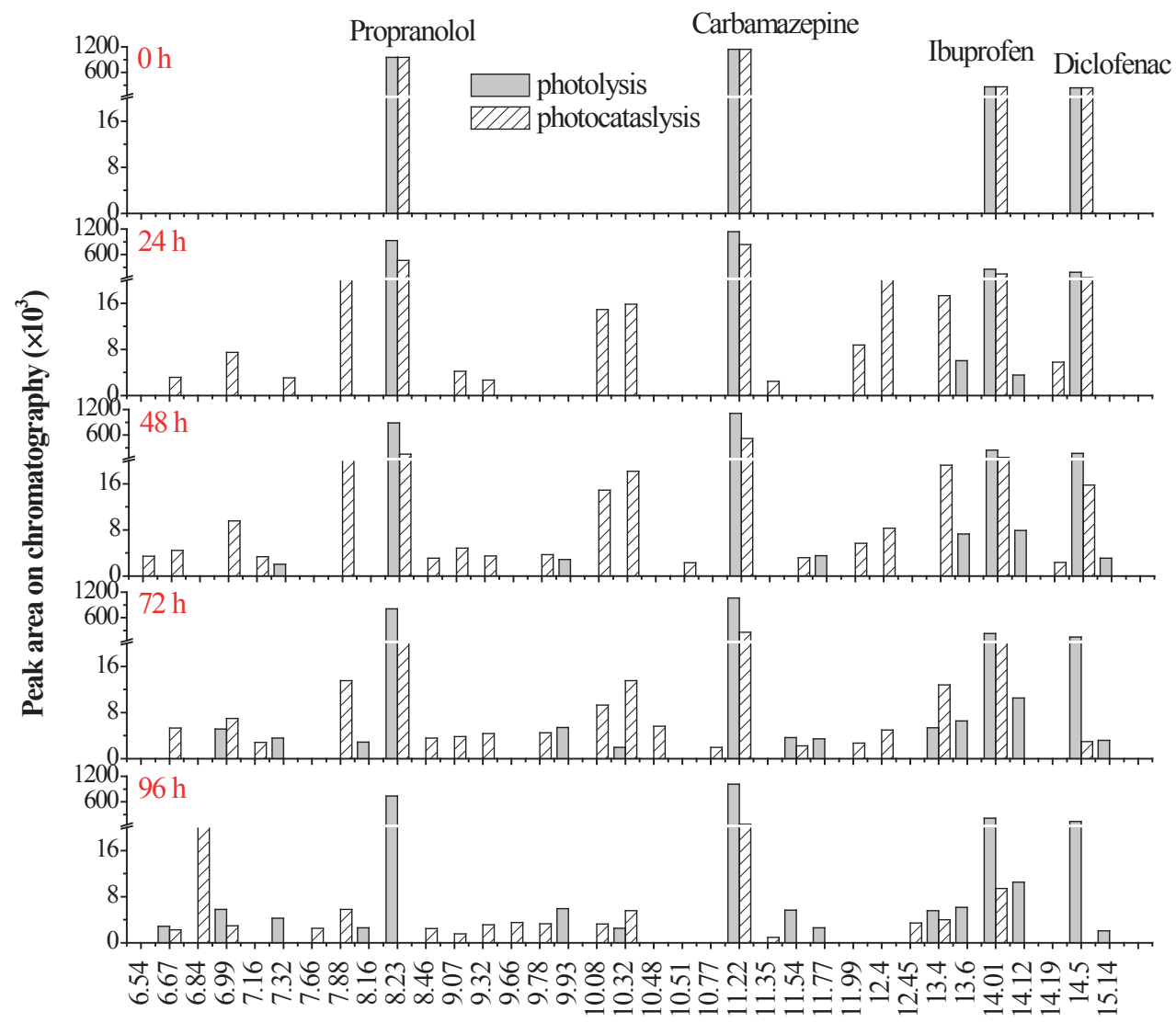

Retention time on chromatography (min)

Figure S3.7 Presence and variation of by-products at different irradiation time points showed in chromatography spectrum. Photolysis and photocatalysis of PhACs spiked in deionized water (DI). 


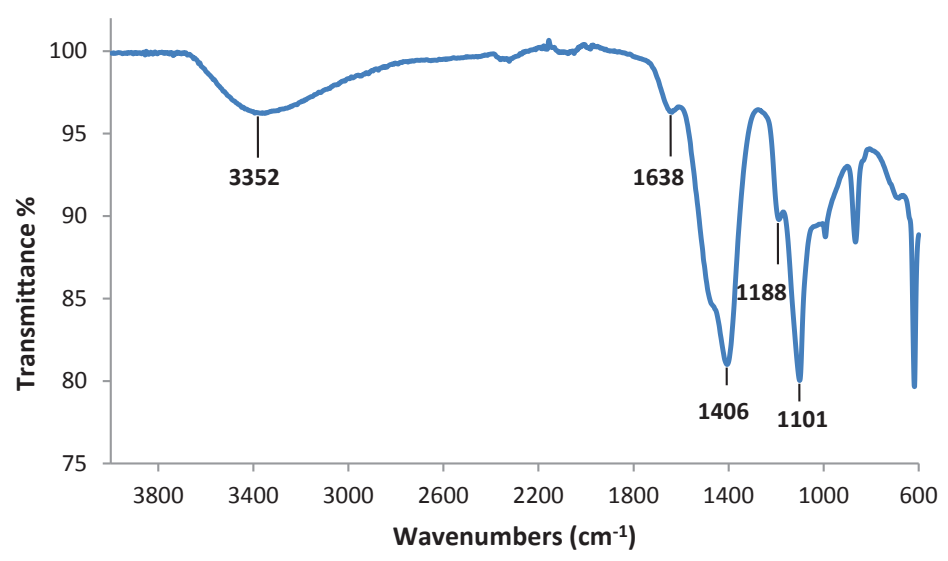

Figure S3.8 FTIR spectra of our wastewater effluent sample. absorption bands corresponding to different functional groups: 1) $3352 \mathrm{~cm}^{-1}$ (O-H stretching of inter- and intra-molecular hydrogens bonds); 2) $1638 \mathrm{~cm}^{-1}$ (aromatic $\mathrm{C}=\mathrm{C}$ skeletal vibrations, asymmetric stretching of $\mathrm{C}=\mathrm{O}$ of quinones and ketones, symmetric stretching of $\mathrm{COO}^{-}$); 3) $1406 \mathrm{~cm}^{-1}$ (asymmetric stretching of $\mathrm{COO}^{-}, \mathrm{C}-\mathrm{H}$ bending of aliphatic groups); 4) $1188 \mathrm{~cm}^{-1}$ (C-O asymmetric stretching and $\mathrm{OH}$ bending of $\mathrm{COOH}$ groups and phenols); 5) $1101 \mathrm{~cm}^{-1}$ (C-O stretching of primary alcohols and in-plane bending of aromatic $\mathrm{C}-\mathrm{H}$.

Table S3.1 Basic water quality data of wastewater effluent of Bennekom wastewater treatment plant $(\mathrm{pH}=7.3)$.

\begin{tabular}{llll}
\hline Components & Concentration $(\mathrm{mg} / \mathrm{l})$ & Components & Concentration $(\mathrm{mg} / \mathrm{l})$ \\
\hline BOD & 6.0 & Chloride & 49.54 \\
COD & 35.2 & Fluoride & not detected \\
DOC & 12.2 & Sulphate & 24.40 \\
Total nitrogen & 7.2 & Nitrate & 4.5 \\
Total phosphate & 0.55 & Nitrite & not detected \\
Kjeldahl-nitrogen & 2.7 & Bromide & not detected \\
\hline
\end{tabular}




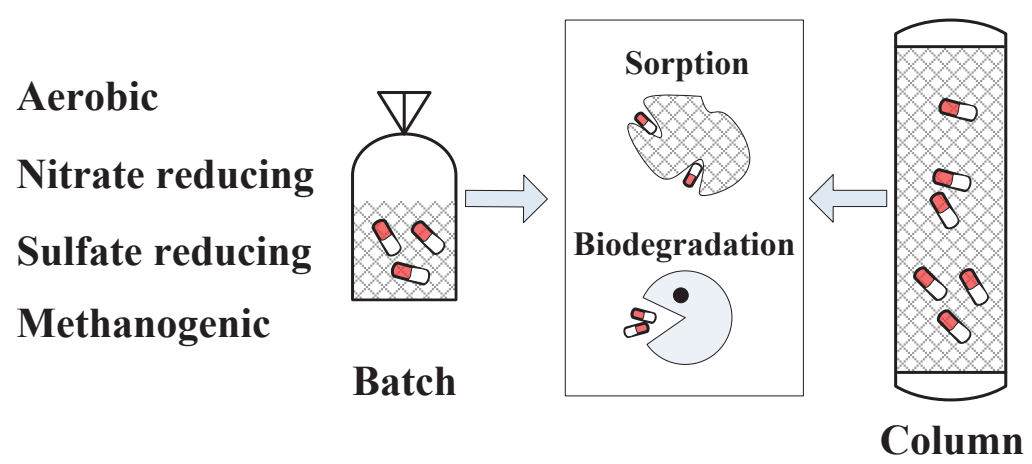




\section{Chapter 4}

\section{Influence of redox conditions on sorption and} biodegradation of pharmaceuticals in sediment batch and column experiments

\footnotetext{
A modified version of this chapter has been submitted as

De Wilt, A. *, He, Y.*, Sutton, N.B., Langenhoff, A.A.M., Rijnaarts, H.H.M. Influence of redox conditions on sorption and biodegradation of pharmaceuticals in sediment batch and column experiments). Chemosphere.

* Both authors contributed equally as the first author.
} 


\begin{abstract}
Specific redox conditions select for specific environmental conditions and microbial communities with different targeted functions, and thereby strongly influence sorption and biodegradation of organic contaminants. This study explored the removal of six pharmaceutically active compounds (PhACs) in constructed wetland sediments under four redox conditions, namely aerobic, nitrate reducing, sulfate reducing, and methanogenic conditions using batch and column set-ups. Our study demonstrates that sorption and biodegradation dominate PhAC removal. Findings of batch experiments are comparable with outcomes of column experiments. For individual PhACs, sorption behaviour is more related to their molecular charge than hydrophobicity. Redox conditions influence both $\mathrm{PhAC}$ sorption and biodegradation. Of the four PhACs which showed sorption, lowest sorption was found under nitrate reducing conditions. Aerobic biodegradation of PhACs was better than anaerobic biodegradation. Among the anaerobic conditions no specific redox condition asserted a more effective overall PhAC biodegradation than the others. Reflecting to PhAC sorption and biodegradation in sediment-based applications, this study shows that breakthrough of all tested PhACs is expected after 300 pore volumes and pre-exposure can improve the PhAC biodegradation ability. In summary, this study provides a comprehensive insight into the influence of redox conditions on the sorption and biodegradation of PhACs in constructed wetland sediments.
\end{abstract}

\title{
Keywords
}

Pharmaceuticals; Redox conditions; Sorption; Biodegradation; Constructed wetland sediment 


\subsection{Introduction}

Pharmaceutically active compounds (PhACs) were developed to target specific human physiological pathways [270]. After consumption, residual PhACs or/and their metabolites are excreted from human bodies into sewage systems. However, conventional wastewater treatment plants (WWTPs) are not specifically designed for removing PhACs [315]. Therefore, PhACs that are not completely removed are discharged to the aquatic environment and may even reach drinking water intakes [53]. Carmona et al. [54] investigated the transport of $\mathrm{PhACs}$ from wastewater to drinking water resources. PhACs were detected in wastewater effluents at concentrations of 7-940 ng/l. Some prevalent PhACs such as ibuprofen ended up in drinking water in the level of approx. $100 \mathrm{ng} / 1$ which is concerning, considering the guideline value of $100 \mathrm{ng} / \mathrm{l}$ micropollutants adopted by toxicologists for safe drinking water [363]. This problem will be exacerbated in the future as PhAC use and discharge will increase, as population increases in urban areas, aging of these populations proceeds, and the accessibility of more people to medical care improves. Thus more PhACs are consumed and PhAC fluxes towards WWTPs and surface waters are increasing [352]. In this context, efficient post-treatment technologies for removing PhACs are needed and emerging.

Commonly proposed physico-chemical post-treatment technologies have various disadvantages. For example, activated carbon traps contaminants in the solid phase, needs to be regenerated, which is costly, and does not capture non-sorbing compounds. Chemical technologies such as UV-treatment and ozonation effectively transform contaminants but also have high operational costs due to expensive equipment and energy demand [3]. In comparison, constructed wetlands (CWs) with adequate removal efficiencies using less energy and base materials have lower operational costs and a self-maintaining capacity. CWs could therefore be a more robust and attractive alternative.

However, processes involved in $\mathrm{CW}$ systems are more complex and require a proper understanding to come to a robust design and operation. In the sediment of CWs, both sorption and biodegradation play an important role in removing organic contaminants. A multitude of factors affect the removal process, such as redox conditions (electron acceptors), organic matter, temperature, and $\mathrm{pH}$ [272]. Especially natural organic matter (NOM), temperature and $\mathrm{pH}$ affect sorption of organic contaminants. However, the effect of redox conditions on sorption behaviour on organic contaminants is unknown. On the contrary for 


\section{Sorption and biodegradation}

biodegradation, specific electron acceptors select for specific microbial communities with different targeted functions, and thereby strongly influence the biological removal of organic contaminants [119]. Maeng et al. [248] studied removal efficiencies of micropollutants in river bank infiltrations and managed aquifer recharges using principle component analysis. They concluded that the redox condition is the main factor dictating variance in removal efficiencies. Specific redox conditions can be engineered to favour and optimize specific microbial respiration processes involved in removing specific pollutants [119]. For example, transformation of sulfamethoxazole was reported to strongly depend on the occurrence of nitrate reducing conditions and be sensitive to the concentration of nitrate [20].

Although redox conditions are identified as one of the controlling factors for removing PhACs, the reported dependencies of removal processes on redox conditions vary significantly for specific PhACs for various reasons. Firstly, most of the previous works only study the removal efficiencies of PhACs under aerobic and anaerobic conditions without identifying the dominant terminal electron acceptor [385, 413]. Secondly, results reported for PhACs biodegradation in terms of redox effects are often contradictory. For example, sulfamethoxazole (SMX) was proved to be more rapidly eliminated under anoxic conditions than under aerobic conditions in bank filtration in the work of Heberer et al. [161], while Baumgarten et al. [29] concluded that SMX was more rapidly removed under aerobic conditions compared to anoxic conditions. Conkle et al. [81] concluded that degradation of carbamazepine (CBZ) was enhanced under aerobic conditions as compared to anaerobic conditions in sediment collected from three types of wetlands; in contrast, Hai et al. [151] reported that $\mathrm{CBZ}$ showed degradation only in an anoxic environment instead of under oxic conditions in a membrane bioreactor. Furthermore, the various studies that report the effects of redox conditions on $\mathrm{PhAC}$ removal are difficult to compare as they use different reactor setups, different concentrations, and different compounds.

Thus, there is a significant knowledge gap on comparative effects of redox conditions on removal of PhACs in CWs. To get a more comprehensive understanding of the influence of selected redox conditions on specific PhACs removal via sorption and biodegradation, it is necessary to investigate this in defined experimental setups varying the applied redox conditions. Therefore, the objective of this study is to elucidate the influence of redox conditions on removal mechanisms of six PhACs applying four specific redox conditions. Batch and column systems were used for controlled tests under aerobic, nitrate reducing, 
sulfate reducing, and methanogenic conditions. The results of this study give insight into understanding the influence of redox conditions on $\mathrm{PhACs}$ removal in $\mathrm{CW}$ systems.

\subsection{Materials and methods}

\subsubsection{Chemicals and regents}

PhACs were purchased from Sigma-Aldrich (USA) at various purity grades: $( \pm$ )-metoprolol $(+)$-tartrate salt (MET, $\geq 98 \%$ ), caffeine (CAF, $\geq 99 \%)$, ( \pm )-propranolol hydrochloride (PRO, $\geq 99 \%$ ), Carbamazepine (CBZ, $\geq 99 \%$ ), naproxen (NAP, $\geq 98 \%$ ), ibuprofen (IBP, $\geq 98 \%$ ), and fenoprofen calcium salt $(\geq 97 \%)$. Physicochemical properties of PhACs are given in Table S2.1. Acetonitrile with $0.1 \%$ formic acid, water with $0.1 \%$ formic acid, and methanol (Biosolve B.V., the Netherlands) were used for ultra-performance liquid chromatography (UPLC) analysis. Stock solutions of PhACs were prepared in deionized water for batch and in methanol for column. All the other chemicals and reagents used were of analytical grade or higher. Deionized water from a Milli-Q system (Millipore, USA) was used to prepare solutions.

\subsubsection{Experimental setup}

\subsubsection{Sediment}

CW sediment was collected from WWTP Hapert and WWTP Land van Cuijk (both, the Netherlands) as a solid phase of the batch and column systems. In addition, the sediments contain microorganisms that serve as a natural inoculant of the biologically active laboratory systems. CWs at both facilities have received WWTP effluent for several years. Sediment dry matter $(\mathrm{DM})$ and organic matter $(\mathrm{OM})$ content were determined gravimetrically after drying at $105{ }^{\circ} \mathrm{C}$ following combustion at $550{ }^{\circ} \mathrm{C}$.

The aerobic column was inoculated with upper layer sediment $(0-5 \mathrm{~cm})$ with an OM content of $6.2 \mathrm{~g} \mathrm{OM} / \mathrm{kg}$ DM. Sediment at a depth of 10-20 $\mathrm{cm}$ below the surface level with an OM content of $16.2 \mathrm{~g} \mathrm{OM} / \mathrm{kg} \mathrm{DM}$ was collected to inoculate the anaerobic columns. A mixture of upper, deeper layer, and rhizosphere sediment was used for batch experiments containing 19 $\mathrm{g} \mathrm{OM} / \mathrm{kg} \mathrm{DM}$. 


\section{Sorption and biodegradation}

\subsubsection{Batch experiments}

Four different media were used to enrich dominant bacteria in different redox conditions. Media were prepared according to previous works for aerobic (Table S4.1), nitrate reducing [112], sulfate reducing [212], and methanogenic conditions [174]. The ionic strength of media was calculated by OLI Studio Analyzer 9.2 software. In each batch bottle, $15 \mathrm{~g}$ wet sediment was mixed with $120 \mathrm{ml}$ medium spiked with mixture of six PhACs (1 mg/l each). The gas phase of each bottle was filled with either atmospheric air for aerobic conditions or $\mathrm{CO}_{2} / \mathrm{N}_{2}(20 / 80, \mathrm{v} / \mathrm{v})$ for anaerobic conditions. Among the four redox conditions, no extra carbon source was added except for the PhACs, spiked into the systems. Abiotic controls contained chemical inhibitors: $1.3 \mathrm{~g} / 1$ of sodium azide in aerobic batch bottles, and $0.3 \mathrm{~g} / 1$ of mercury chloride in anaerobic bottles, which are concentrations known to control abiotic conditions. The aerobic batch experiment lasted for six weeks and samples were collected on week $0,1,3$ and 6; the anaerobic batch lasted for three months and samples were collected every three weeks. Samples of week 0 were collected the day after spiking to ensure a homogeneous distribution of PhACs. In order to determine the role of microbial adaptation, mixed PhACs were re-spiked three times in the aerobic batch for enrichment after week 6 . Batch bottles were incubated on a shaker $(120 \mathrm{rpm})$ at $20^{\circ} \mathrm{C}$. To prevent photolysis batch bottles were kept in the dark during the experiment. Sorption coefficient $\mathrm{K}_{\mathrm{d}}$ was calculated from the abiotic controls as the ratio of the PhACs concentration in the sediment phase and in the water phase at equilibrium. The concentrations of sorbed PhACs were calculated from the measured water phase concentrations based on mass balance. 


\subsubsection{Column experiments}

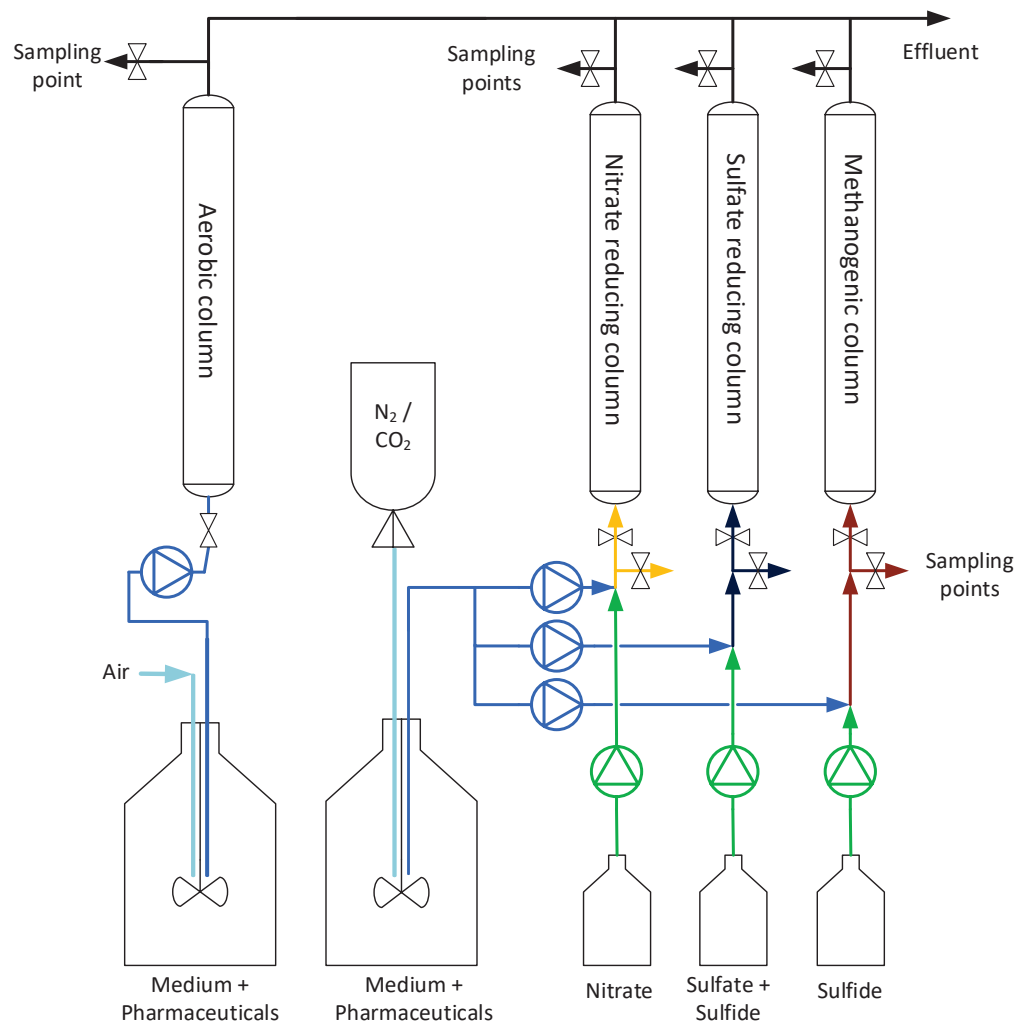

Figure 4.1 Experimental set-up of the column experiments.

Column experiments were conducted in four continuous-fed upflow soil columns (Figure 4.1). Identical to the batch experiments, aerobic, nitrate reducing, sulfate reducing and methanogenic conditions were tested. Cylindrical glass columns $(0.23 \mathrm{~L})$ were packed with wet sediment containing 292 g DM and 230 g DM for respectively aerobic and anaerobic experiments. Sediment was retained in the columns by sintered glass filters (pore size 40-100 $\mu \mathrm{m})$. The packed sediment was circularly homogenised biweekly, while keeping sediment layers at their depths in the columns. This is needed to prevent bypass flows through the columns, i.e. as a result of mobilised particle clogging or biogas bubbles formed. Columns were fed with medium at a flow rate of $9.8 \pm 0.4 \mathrm{ml} / \mathrm{h}$, resulting in a hydraulic retention time of $8.2 \pm 0.3 \mathrm{~h}$. For each redox condition, specific media were prepared (Table S4.1), which contained $100 \mu \mathrm{g} / \mathrm{l} \mathrm{PhACs}$ during the experiment of 110 days. Prior to the experiment columns were run two weeks on media without $\mathrm{PhACs}$ to allow redox conditioning. Influent 


\section{Sorption and biodegradation}

and effluent samples were collected for chemical analysis within 24 hours from each other per time point. PhAC removal was calculated based on effluent concentrations per time point over the averaged influent concentration of the entire experiment to deal with fluctuations in the influent concentration. Sorption parameter values (specific sorption coefficient $\mathrm{k}_{\mathrm{d}}$ ) were determined for sorptive PhACs by calculating the retardation factor $\left(\mathrm{R}_{\mathrm{d}}\right)$ from the time point when the effluent PhAC concentration reached fifty percent of the influent concentration. Columns and media were kept in the dark during the experiment to prevent photolysis.

\subsubsection{Sample collection and analysis}

Liquid samples were taken from batch media and column influents and effluents for PhACs measurements. Samples collected from batch bottles were centrifuged at 10,000 rpm for 10 min (Microlite, Thermo IEC, USA) and stored at $-20{ }^{\circ} \mathrm{C}$ prior to analysis. Liquid samples of column experiments were pre-treated by solid phase extraction (SPE) before analysis. Oasis HLB cartridges (6 cc/60 mg, Waters, USA) were pre-conditioned with $5 \mathrm{ml}$ methanol and equilibrated with $5 \mathrm{ml}$ buffered deionized water (10 $\mu \mathrm{l}$ buffer/ml, $\mathrm{pH}=10$, Merck, Germany). Cartridges were loaded with $3 \mathrm{ml}$ and $9 \mathrm{ml}$ for influent and effluent samples, respectively. Loaded cartridges were washed with $5 \mathrm{ml}$ buffered deionized water and eluted with $10 \mathrm{ml} 25 \%$ $\mathrm{NH}_{4} \mathrm{OH}$ : methanol $(8 / 92, \mathrm{v} / \mathrm{v})$. Eluates were evaporated till dryness under a gentle nitrogen flow. Samples were reconstituted in $1 \mathrm{ml} 3.6 \%$ methanol. Prior to SPE, 10,11dihydrocarbamazepine was spiked to samples as an internal standard. PhAC analysis was conducted by UPLC (Ultimate 3000, Thermo, USA) as described by He et al. [156]. Quantification was based on the internal standard and external calibration standards. Results were considered valid if SPE recoveries were within $85-115 \%$.

Concentrations of nitrite, nitrate, and sulfate were measured by ion chromatography (IC). Before measurement, samples were filtered using a $0.45 \mu \mathrm{m}$ cellulose filter (VWR, USA) and diluted five times with MilliQ water. A Dionex ICS-2100 IC system (Thermo, USA) was used for analysis. The system was equipped with an anion exchange column (Dionex, IonPac AS19, $4 \times 250 \mathrm{~mm}$ ), where the anions were separated using a hydroxide gradient. The eluent was made automatically using the eluent generator configured with a $\mathrm{KOH}$ cartridge (Dionex $\mathrm{P} / \mathrm{N}$ 058900) and deionized water as the carrier. Detection was done by a DS6 Heated conductivity cell.

Oxygen and methane were analysed in the headspace of batches by gas chromatography (GC2010, Shimadzu, Japan). The instrument contained a parallel combination column: Porabond 
$\mathrm{Q}(50 \mathrm{~m} \times 0.53 \mathrm{~mm} ; 10 \mu \mathrm{m})$ and Molsieve $5 \mathrm{~A}(25 \mathrm{~m} \times 0.53 \mathrm{~mm} ; 50 \mu \mathrm{m})$. The carrier gas was helium and operated at 0.95 bar. Column temperature was $80{ }^{\circ} \mathrm{C}$, detector temperature was $150{ }^{\circ} \mathrm{C}$, and injection temperature was $120^{\circ} \mathrm{C}$. Pressure in the batch was measured by a digital pressure meter (GMH 3151, Greisinger) for calculating gas volume. In the aerobic column dissolved oxygen was measured by optical oxygen meter Fibox 3 trace (PreSens, Germany) fixed near the outlet of the column. For methanogenic column experiments, dissolved methane in liquid effluent samples was turned into gaseous methane as described by Zhang et al. [403] and analysed by GC as described above.

\subsection{Results}

\subsubsection{Redox conditions}

Consumption of electron acceptors and accumulation of respiration products were observed in all batches and columns (Figure S4.1). Under aerobic conditions, $\mathrm{O}_{2}$ consumption was observed. Nitrate and sulfate consumption were detected under nitrate and sulfate reducing conditions, respectively. Production of $\mathrm{CH}_{4}$ was observed under methanogenic conditions. These results indicated that the desired redox conditions were achieved. In addition, changes in sediment colour were observed in both batch and column experiments. Sediment in the aerobic and nitrate reducing conditions turned lighter after several days of operation, indicating that applied redox conditions had an influence on the sediment.

\subsubsection{Removal of PhACs}

The removal of PhACs in biological systems can be attributed to biodegradation, chemical conversion, sorption, volatilization, and photolysis. The chosen experimental setup is aimed to parse out the contribution of physical, chemical, and biological processes to $\mathrm{PhAC}$ removal. The selected compounds are considered non-volatile and experiments were performed in dark, therefore volatilization and photolysis of PhACs are considered to have no effect in this work. To our knowledge, PhACs have not been reported to be chemically converted in sediments. We demonstrated that PhACs did not react with batch and column media (results not shown). The removal of PhACs is therefore hypothesized to be mainly influenced by sorption and biodegradation. 


\section{Sorption and biodegradation}

\subsubsection{Batch systems}

Outcomes from abiotic controls are used to characterize the sorption capacity of the used sediment for targeted PhACs. To compare PhAC removal under different redox conditions, $\mathrm{PhAC}$ removal under aerobic conditions was only shown in the first six weeks as PhACs were re-spiked afterwards. In general, the high sorption capacity of CW sediment was found for NAP, CAF, PRO, and MET while CBZ and IBP were much less sorbed, especially in sulfate reducing and methanogenic conditions (Figure 4.2). Sample of week 0 was collected after mixing the bottles overnight, which might cause fast sorption and then followed by desorption resulting in negative removal. When comparing PhAC sorption under different redox conditions, nitrate reducing conditions showed lower sorption capacity for MET, NAP, and CAF compared with sulfate reducing and methanogenic conditions.

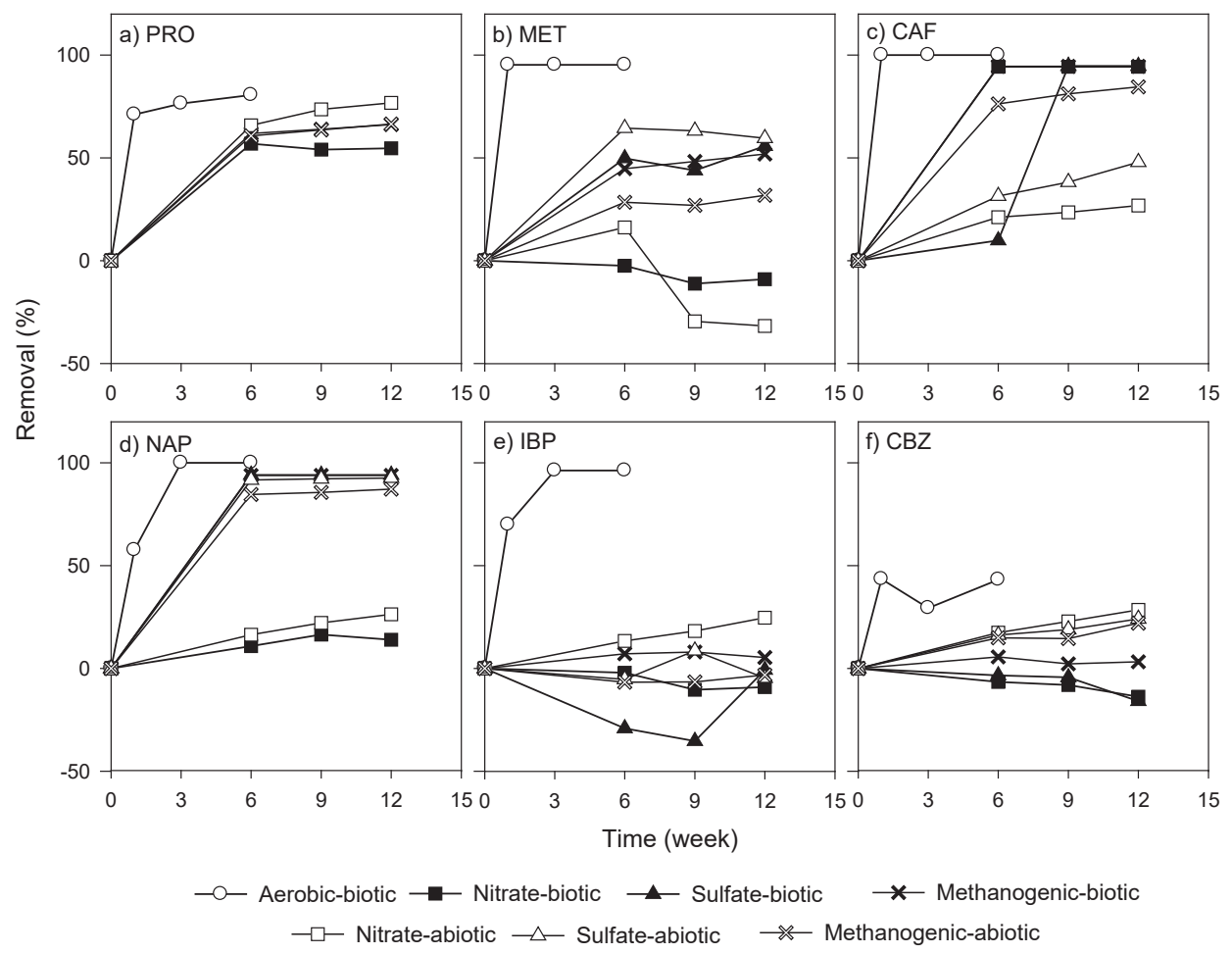

Figure 4.2 Removal of PhACs under different redox conditions in batches: a) PRO; b) MET; c) CAF; d) NAP; e) IBP; f) CBZ. PRO removal under sulfate reducing conditions is not shown because the initial PRO concentration was accidently very low $(0.05 \mathrm{mg} / \mathrm{l})$ under sulfate reducing condition, which is the same as the detection limit. Abiotic removal of PhACs under aerobic conditions per time point is not shown and abiotic removal at endpoints are given in Table S4.2. 
Biodegradation in batches was determined by comparing biotic and abiotic removal efficiencies. Under aerobic conditions, MET, CAF, NAP, and IBP were completely removed within 6 weeks (Figure 4.2), which was mainly contributed to biodegradation as sorption only contributed to less than $30 \%$ (Table S4.2). In comparison, PRO and CBZ were poorly biodegraded considering their similar biotic and abiotic removal. Under anaerobic conditions, only CAF was readily biodegraded under nitrate and sulfate reducing conditions. Removal of CAF under methanogenic conditions was mainly caused by sorption (Figure 4.2). Similarly, the observed removal of PRO, NAP, and MET was a result of their high sorption rather than biodegradation. Under all anaerobic conditions IBP and CBZ were poorly removed in the biotic and abiotic batches.

To determine the role of microbial adaptation, the aerobic inoculum in batches was enriched to select for PhACs removal by re-spiking PhACs. During the first six weeks, NAP and IBP were approximately $60-70 \%$ removed within one week and complete removal was reached at week 3 (Figure 4.3). After re-spiking, complete removal of NAP and IBP was reached within one week (Figure 4.3). Therefore, microbial adaptation by pre-exposure did accelerate the biodegradation of NAP and IBP. Half-lives of NAP and IBP were 1.0 and 1.8 days; half-lives of MET and CAF were less than 1 day (Figure S4.2).

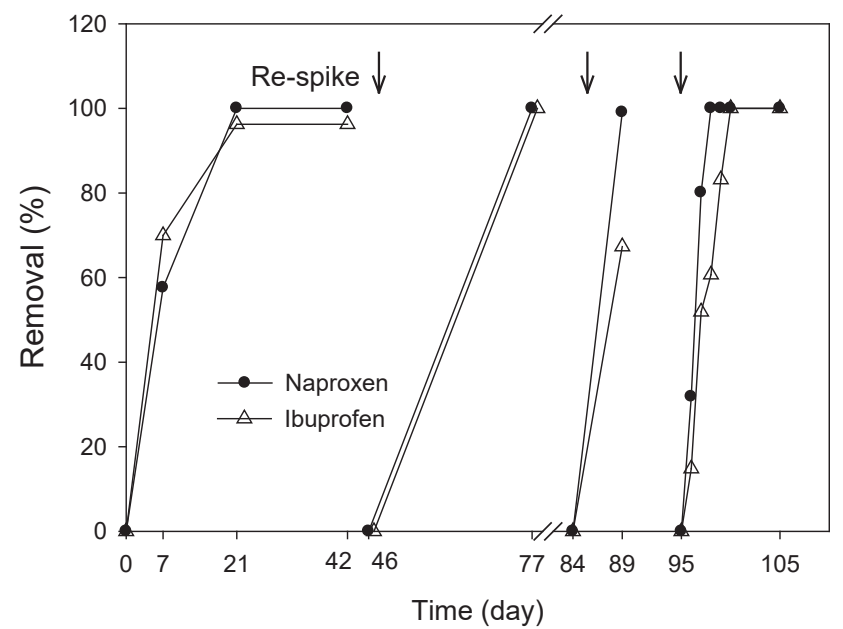

Figure 4.3 Removal of NAP and IBP under aerobic condition with re-spike of PhACs at day 46, 84, and 95 . 


\section{Sorption and biodegradation}

\subsubsection{Column systems}

Different removal patterns were observed for individual PhACs in columns among the tested redox conditions (Figure 4.4). In general the PhAC removal patterns could be typically described as 1) high removal followed by breakthrough of the PhAC, 2) high removal with subsequent breakthrough followed by an increase in removal, 3) low to moderate removal followed by an increase in removal, 4) constant high removal and 5) no removal. Main removal mechanisms were interpreted based on the observed removal patterns. Sorption was indicated to be a dominating removal process when initial $\mathrm{PhAC}$ removal was followed by breakthrough of that compound. Biodegradation was identified by the presence of a lag phase, significant removal and absence of subsequent breakthrough behaviour. In the case of IBP and CBZ for which no significant sorption or biodegradation was observed in any of the columns the PhACs were classified as persistent (Figure 4.2).

Significant removal (>95\%) of PRO and MET followed by compound breakthrough was apparent under all redox conditions (Figure 4.4). Therefore, sorption was identified to be the dominant removal mechanism for PRO and MET. Sorption of PRO and MET appears to be affected by the applied redox conditions. Methanogenic and sulfate reducing conditions indicated better sorption compared to aerobic and nitrate reducing conditions for PRO and MET. Especially the removal of MET under nitrate reducing conditions was limited compared to the other anaerobic redox conditions whereas the sediment was identical.

CAF appeared initially to be removed by sorption in each column (65-99\%) (Figure 4.4). However, the sorption capacity of the sediment towards CAF was low compared to PRO and MET as breakthrough behaviour was observed already after day 4 in all columns. Under all redox conditions, biodegradation of CAF took place after a lag phase: under aerobic and nitrate reducing conditions the lag phase lasted 27 days, while only after 67 days an increase in removal was found in the sulfate reducing and methanogenic columns.

The removal of NAP showed significant differences among the tested redox conditions. Complete NAP removal ( $>99 \%$ ) was achieved in the aerobic column after a lag phase of 27 days. The presence of the lag phase followed by the continuously high removal in the aerobic column indicates that biodegradation was the main removal mechanisms for NAP under aerobic conditions. Insignificant NAP removal $(<25 \%)$ was observed under nitrate reducing conditions (Figure 4.4). In the sulfate reducing and methanogenic columns, a constant NAP removal throughout the experiment of around $80 \%$ was found. This phenomenon could not be 
attributed to sorption, as there was neither breakthrough of NAP nor a constant complete NAP removal. Chemical NAP removal by the reactor medium was also excluded as it was investigated (results not shown). Therefore, most likely biodegradation is the main removal mechanism for NAP under sulfate reducing and methanogenic conditions. Why the removal did not improve over time, as would be expected due to the growth of more specialized NAP degrading microorganisms in a biological system, is not fully understood. A reason could be that microbial growth of specialized NAP degrading microorganisms is limited, i.e. by a growth factor limitation.

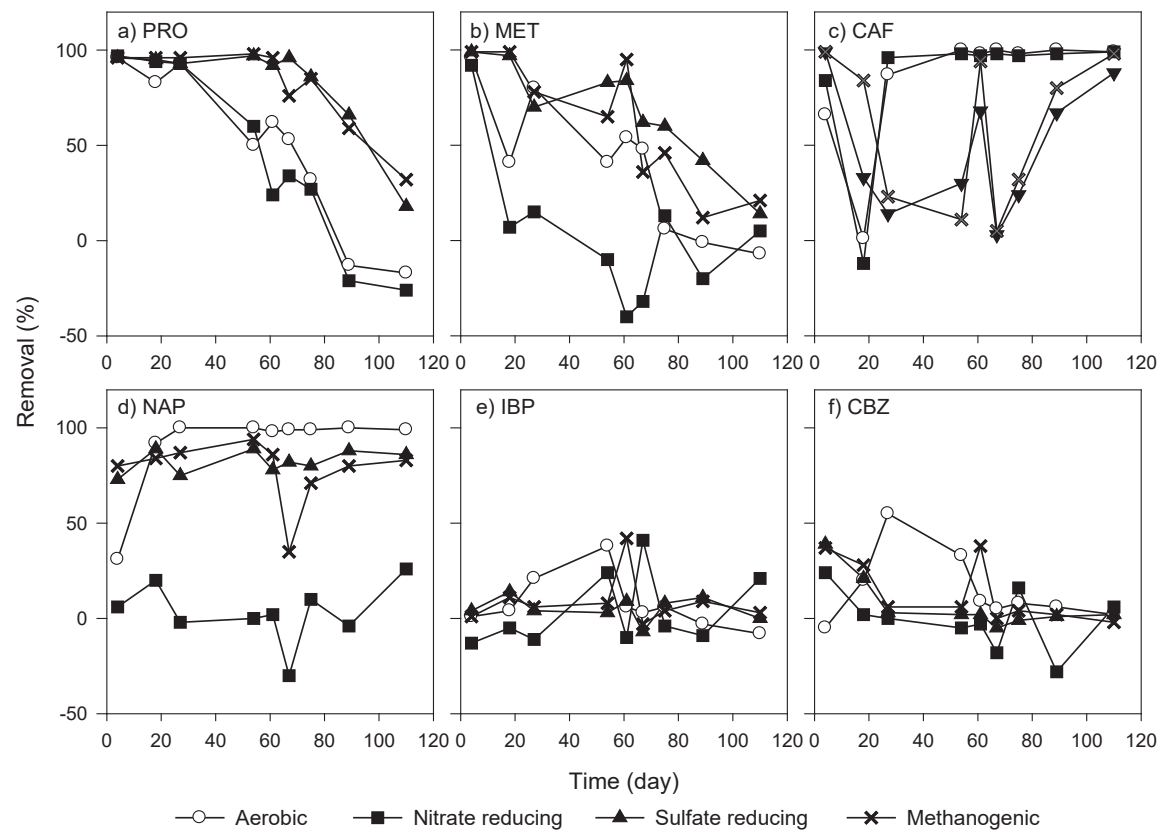

Figure 4.4 Removal of PhACs under different redox conditions in continuous flow-through columns: a) PRO; b) MET; c) CAF; d) NAP; e) IBP; f) CBZ.

\subsection{Discussion}

\subsubsection{Sorption of PhACs}

In both batches and columns, stronger sorption was indicated for PRO, MET and CAF while CBZ and IBP showed no significant sorption. NAP was highly sorbed in batches under sulfate reducing and methanogenic conditions and not in columns. In batches, sorption coefficient $\mathrm{K}_{\mathrm{d}}$ was calculated under all anaerobic conditions for all PhACs. In columns the 


\section{Sorption and biodegradation}

breakthrough behaviour of PRO, MET and CAF allowed the calculation of the retardation factor and $K_{d}$. These $K_{d}$ values were compared to available literature data on the sorption behaviour of target $\mathrm{PhACs}$ in agricultural soils, aquifer sediments, river sediments and $\mathrm{CW}$ sediments (Figure 4.5). Coefficients from literature demonstrate that PRO, CAF and MET sorbed more readily than NAP, CBZ, and IBP (in median, Figure 4.5). $\mathrm{K}_{\mathrm{d}}$ values found in this study are in accordance with reported literature values, except for MET in columns.

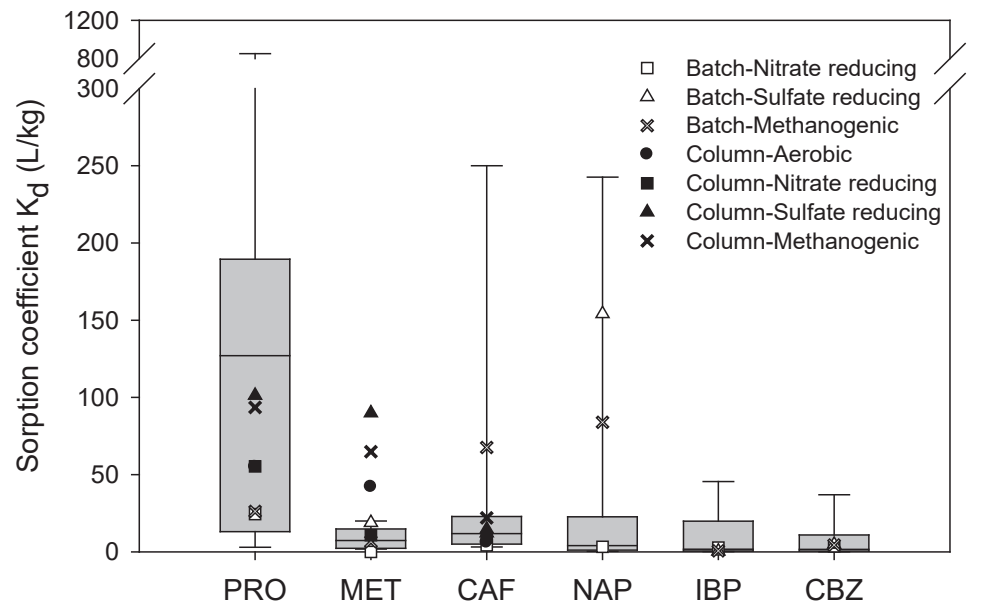

Figure 4.5 Comparison of sorption coefficients $K_{d}$ between this study and literature $[24,81,97,106$, 230, 251, 256, 300, 305, 317, 368, 381, 386, 406, 410]. For literature values of PRO, MET, CAF, NAP, IBP and CBZ, $\mathrm{n}=12,7,6,15,19$, and 8 , respectively. The box plot shows the values found in literature in maximum, third quartile, median, first quartile, and minimum. The squares, triangles, crosses and circles represent the values for the different redox conditions in batch (no filling) and column (filled).

Sorption of PhACs was reported to depend on their physicochemical properties, such as hydrophobicity and molecular charge [48]. In this study, we found that PhAC sorption by the sediment is more related to the molecular charge of PhACs than their hydrophobicity. To characterize the hydrophobicity, the apparent partitioning coefficient $\log \mathrm{D}_{\mathrm{ow}}$ (Table S2.1) was applied to modify the octanol-water partition coefficients $\log \mathrm{K}_{\mathrm{ow}}$ with $\mathrm{pH}$ [217]. However, PhACs sorption did not correlate with $\log \mathrm{D}_{\mathrm{ow}}$ (Figure S4.3). Based on $\mathrm{pK}_{\mathrm{a}}$ values (Table S2.1) and the $\mathrm{pH}$ range in our study $(\mathrm{pH}=6.8-7.5), \mathrm{CBZ}$ has no charge, IBP and NAP are negatively charged, while the other three PhACs are positively charged. Most sediment matrix components are negatively charged $[251,386]$. This likely explains why the cationic 
PhACs (CAF, PRO, MET) showed a stronger tendency to sorb compared to anionic (IBP) and neutral species (CBZ). The high sorption of anionic NAP in batches might be caused by the NOM present in the sediment. Similar results were observed in the work of MartínezHernández et al. [251]. These authors found that sorption onto the inorganic surface of sediment was the predominant sorption mechanism for all charged PhACs examined with the exception of NAP, which was partitioned to NOM instead. The higher content of NOM in batches in this study might explain its higher sorption compared to the columns.

In addition to the effect of molecular charge, it was also observed that redox conditions influence the sorption capacity of sediment towards PhACs. Anaerobic column experiments were inoculated with the same inoculum. Similarly, all anaerobic batches were inoculated with the same sediment. However, different PhAC sorption coefficients were found under different anaerobic redox conditions in batches and columns (Figure 4.5). Sorption of NAP in batches was found to be lowest under nitrate reducing conditions and highest under sulfate reducing conditions. Similar phenomenon was found for PRO sorption in columns and MET sorption in both systems that followed the order nitrate reducing $<$ methanogenic $<$ sulfate reducing conditions. In both systems, sorption of CAF followed the order nitrate reducing < sulfate reducing $<$ methanogenic conditions. Sorption coefficients $K_{d}$ of MET and CAF under different redox conditions correlated between batches and columns (Figure 4.6), which indicate consistency among the two test systems often used in compound removal testing. The sorption difference under various anaerobic redox conditions could not be explained by the characteristics of the media we applied, such as ionic strength. Among all PhACs investigated in batches, only CAF sorption showed to be inversely correlated to ionic strength with $149.5,108.1$ and $63.8 \mathrm{mmol} / 1$ under nitrate reducing, sulfate reducing and methanogenic conditions, respectively. However, the correlation identified for CAF in batches was not observed in columns, where ionic strength are similar under three anaerobic conditions (50.4, 69.3, and $42.6 \mathrm{mmol} / 1$ under nitrate reducing, sulfate reducing and methanogenic conditions, respectively). As the effect of redox conditions on sorption behaviour of $\mathrm{PhACs}$ is not described before, further studies need to be conducted to understand the underlying mechanisms. 


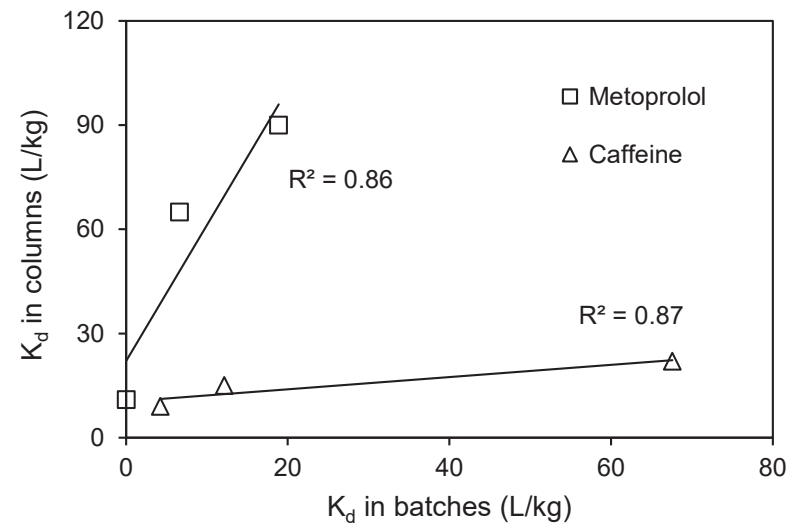

Figure 4.6 Linear relationship of sorption coefficients between batch and column experiments under different anaerobic redox conditions.

Sorption coefficients $\mathrm{K}_{\mathrm{d}}$ in our column study show that breakthrough is expected in sediment based applications. For the most sorptive PhAC, PRO in this study, saturation of sediment sorption sites occurred after approximately 300 pore volumes under sulfate reducing conditions. Rami et al. [300] demonstrated that sorption isotherms of PRO and MET to river and stream sediments are linear between 2-200 $\mu \mathrm{g} / \mathrm{l}$. For linear sorption behaviour retardation factors are independent on the PhAC concentration. Thus, reflecting on sediment based applications treating PhACs in WWTP effluents that are generally in the same concentration range, the sorption capacity of sorptive PhACs like PRO will reach saturation after approximately 300 pore volumes.

\subsubsection{Biodegradation of PhACs}

Aerobic batch and column experiments both demonstrated an effective CAF and NAP biodegradation. Their significant biodegradation is consistent with their biodegradation in soil columns reported by Kim et al. [197]. Additionally, in batches IBP and MET were biodegraded, whereas in column no significant IBP removal was observed and MET was removed by sorption. The poor removal of IBP is contrary to what other authors reported $[190,210]$. The applied hydraulic retention time of 8 hours is in a similar range of 3-18 hours for which IBP removal is reported [114], and seems therefore long enough. Zwiener and Frimmel [413] concluded that IBP is primarily degraded under aerobic conditions and poorly in the absence of oxygen in biofilm reactors. Throughout our experiment, no dissolved oxygen (DO) was found in the aerobic column effluent as it was completely consumed in the 
column. Hence, it is assumed that competition for DO resulted in a too low DO concentration to support significant aerobic IBP biodegradation in columns. MET was biodegraded and sorbed in batches whereas sorption was the only removal mechanisms in column. Biodegradation and sorption are reported to be the main MET removal mechanisms in water/sediment systems [228]. Similar to IBP removal in column, DO might be a limiting factor in MET biodegradation in column which was also observed by Radke and Maier [299]. These authors proved for a sequencing anaerobic-aerobic water/sediment system that MET biodegradation only occurred under aerobic conditions. No significant biodegradation of PRO was found in batches and columns which is comparable to the findings of Radke and Maier [299]. In their study on the removal of PRO in various river sediments, biodegradation occurred in 3 out of 8 tested sediments, while sorption was identified as major PRO removal process in all sediments.

Anaerobic batch and column experiments both demonstrated an effective CAF biodegradation. To our knowledge, there is a lack of studies on CAF biodegradation under anaerobic conditions in sediment. However, it was reported that CAF was $99 \%$ biodegraded in the anaerobic tank of a WWTP [385]. Among the anaerobic conditions, NAP biodegradation was observed in the sulfate reducing and methanogenic columns. In sulfate reducing and methanogenic batches NAP biodegradation could not be confirmed, as NAP removal was mainly ascribed to sorption. PRO was not biodegraded in anaerobic batch or column experiments. Similarly, Popple et al. [294] demonstrated that biodegradation was a minor process for radiolabelled ${ }^{14} \mathrm{C}$-PRO removal in an aerobic/anoxic sequencing batch reactor and Barbieri et al. [22] found no biodegradation of PRO in anaerobic soil column experiments. CBZ was not effectively biodegraded under any redox condition in both batch and column experiments. Similarly, Falås et al. [115] reported no CBZ removal under aerobic, sulfate reducing and methanogenic conditions in lab-scale biological wastewater treatment tests.

In our study using well-defined redox conditions, batch and column experiments independently showed that biodegradation of PhACs was influenced by the applied redox conditions. First, aerobic conditions showed better biodegradation performance compared to anaerobic conditions. In batch experiments, NAP, MET and IBP were readily biodegradable under aerobic conditions while they were recalcitrant under anaerobic conditions. A similar conclusion was identified for IBP biodegradation by Conkle et al. [81]. Half-lives of IBP were 7-19 days or more than 7 months under aerobic or anaerobic conditions, respectively. In 


\section{Sorption and biodegradation}

column experiments, NAP and CAF were significantly biodegraded under aerobic conditions while NAP was not removed under nitrate reducing conditions and CAF removal only started after a two month lag phase under sulfate reducing and methanogenic conditions (Figure 4.4). Secondly, among the anaerobic conditions no specific redox condition was found to assert a more effective overall $\mathrm{PhAC}$ biodegradation than the other anaerobic conditions. According to previous research on anaerobic biodegradation of fuel hydrocarbons which also contain aromatic rings like $\mathrm{PhACs}$, nitrate and sulfate appeared to be the most preferred electron acceptors for degrading toluene, ethylbenzene and xylenes [337]. Nevertheless, in our study NAP was poorly biodegraded under nitrate reducing conditions in the column, while it was effectively biodegraded under the other two anaerobic redox conditions. In contrast, nitrate reducing conditions showed the best biodegradation of $\mathrm{CAF}$, while its biodegradation was hampered under the other two anaerobic conditions in both batch and column experiments.

Pre-exposure to PhACs can improve the biodegradation capacity of sediments towards PhACs. Batch experiments in this work showed a better removal for IBP, NAP, CAF and MET, compared to half-life times reported in literature [81, 231, 406]. The improved removal can be explained by the exposure of the $\mathrm{CW}$ sediments to PhACs prior to the laboratory scale experiments. Sediments used in this study were collected from CWs receiving secondary effluents of WWTPs treating domestic wastewater which were in operation for more than 15 years. We detected the target PhACs in the CW influents in the range of 109-5272 ng/l which demonstrates that the $\mathrm{CW}$ sediments have been exposed to PhACs. It is widely accepted that adaptation of microorganisms to compounds can significantly improve biodegradation rates [332]. Especially for microbes capable of degrading micropollutants, pre-exposure to the compounds can enhance their biodegradation ability [56, 260]. Enrichment experiments by re-spiking PhACs in batch further improved the biodegradation rates of IBP and NAP. Thus, the significance of microbial growth by pre-exposure to $\mathrm{PhAC}$ resulting in enhanced biodegradation was further confirmed.

\subsection{Conclusions}

In conclusion, this study allowed a better understanding of the influence of redox conditions on the processes underlying removal of PhACs. The results show that PhAC removal efficiencies are influenced by the applied redox conditions that influence both sorption and biodegradation. Differences in sediment sorption coefficients $\mathrm{K}_{\mathrm{d}}$ of MET, CAF and PRO among the different redox conditions evidence the effect of redox conditions on sorption. 
Biodegradation of CAF and NAP in two independently tested systems, batch and column, confirm the influence of redox conditions on biodegradation. Among the four tested redox conditions aerobic conditions demonstrated the highest PhAC removal efficiencies with significant biodegradation of IBP, NAP, CAF and MET and sorption of PRO and MET. In addition to redox conditions, molecular charges of PhACs were found to influence sorption behaviour whereas hydrophobicity and ionic strength did not have an effect on PhAC sorption. Saturation of the sediment sorption capacity in sediment based applications treating $\mathrm{PhACs}$ is expected within approximately 300 pore volumes for the most sorptive compounds in this study. Enrichment of biomass by pre-exposure to PhACs in bioactive soil, sediment or other porous media filters is an effective strategy to increase removal efficiencies for a number PhACs. This study provides insights into the importance of redox conditions in developing and designing CWs for PhACs treatment.

\section{Acknowledgement}

Authors thank Yang Jiang and Linda Verweij for their practical support during the experiments. The support provided by China Scholarship Council (CSC) for the research of Yujie He at Wageningen University is kindly acknowledged. 


\section{Supplementary materials}
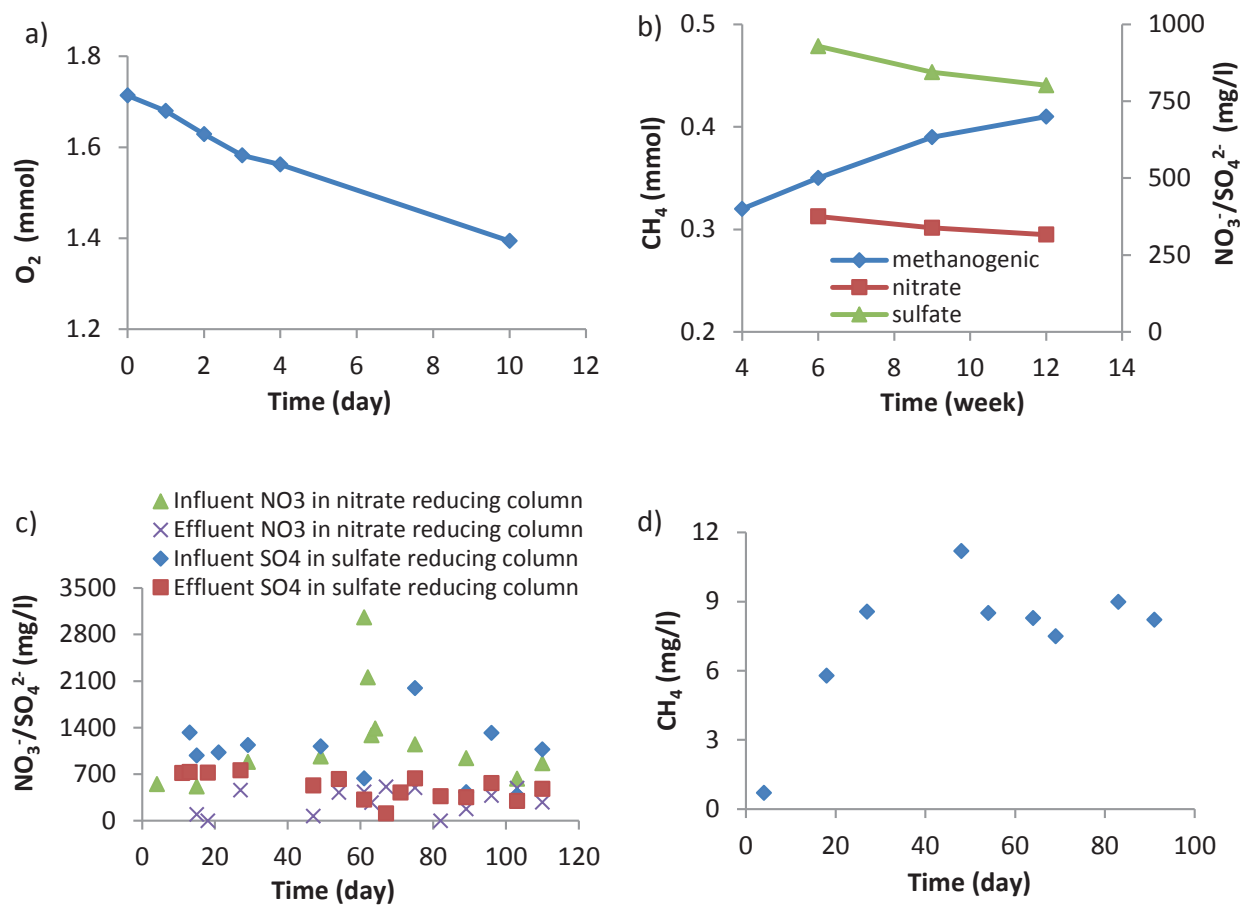

Figure S4.1 Concentrations of electron acceptors and reaction products. a) $\mathrm{O}_{2}$ concentration in aerobic batch experiment; b) $\mathrm{CH}_{4}, \mathrm{NO}_{3}{ }^{-}$, and $\mathrm{SO}_{4}{ }^{2-}$ concentrations in anaerobic batch experiment; c) influent and effluent $\mathrm{NO}_{3}{ }^{-}$and $\mathrm{SO}_{4}{ }^{2-}$ concentrations in columns with nitrate and sulfate; d) effluent $\mathrm{CH}_{4}$ concentration in methanogenic column. 


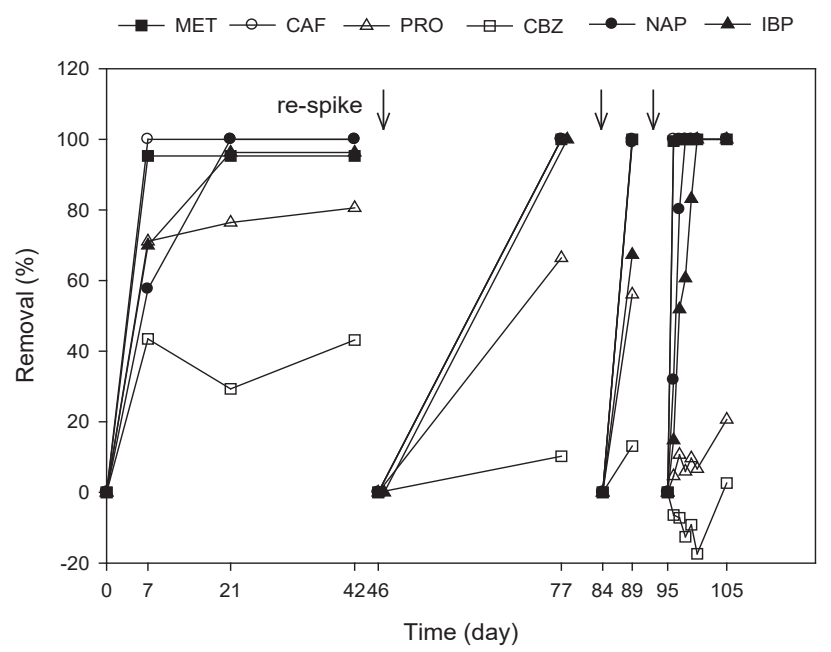

Figure S4.2 Removal of pharmaceuticals under aerobic conditions. PhACs were re-spiked three times at day 46,84 , and 95 .

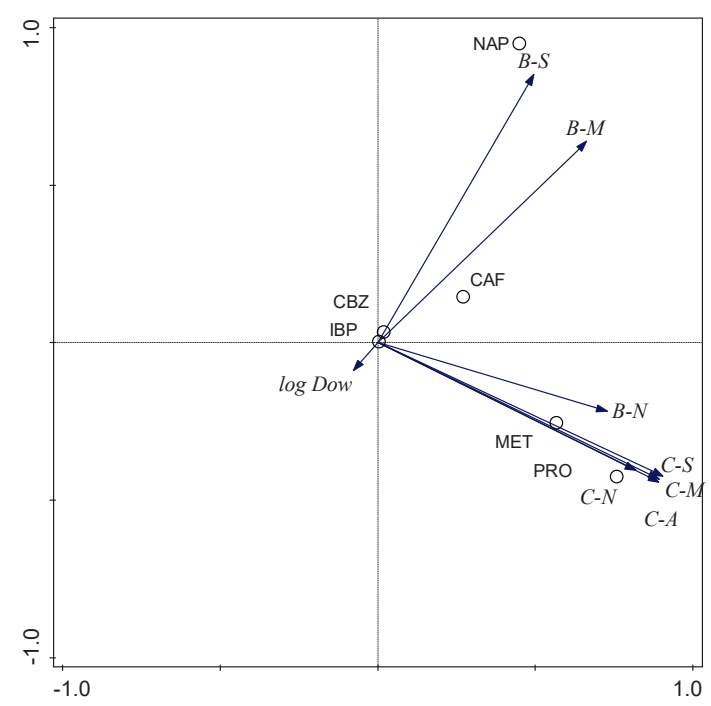

Figure S4.3 Principle component analysis of the relationship between $\log \mathrm{D}_{\text {ow }}$ of PhACs and their $\mathrm{K}_{\mathrm{d}}$ under the applied redox conditions. B-N, B-S, and B-M represent nitrate reducing, sulfate reducing, and methanogenic conditions in batches; C-A, C-N, C-S, and C-M represent aerobic, nitrate reducing, sulfate reducing, and methanogenic conditions in columns. The eigenvalues of the first and second canonical axis are 0.56 and 0.39 . The intersection angles between arrows represent their correlations, in which a more acute intersection angle means stronger correlations. The results show that $\log \mathrm{D}_{\text {ow }}$ of $\mathrm{PhACs}$ is not correlated with the $\mathrm{K}_{\mathrm{d}}$ value under any applied redox conditions. 


\section{Sorption and biodegradation}

Table S4.1 Medium composition for columns and aerobic batches, adjusted from Lindeboom et al. [232].

\begin{tabular}{|c|c|c|c|}
\hline & Reactor & Compounds & $\begin{array}{l}\text { Concentration in } \\
\text { medium }(\mathrm{mg} / \mathrm{L})\end{array}$ \\
\hline \multirow{3}{*}{ Macro nutrients } & \multirow{3}{*}{$\begin{array}{l}\text { Aerobic batches and } \\
\text { columns }\end{array}$} & $\mathrm{NH}_{4} \mathrm{Cl}$ & 1020 \\
\hline & & $\mathrm{CaCl}_{2} \cdot 2 \mathrm{H}_{2} \mathrm{O}$ & 48 \\
\hline & & $\mathrm{MgSO}_{4} .7 \mathrm{H}_{2} \mathrm{O}$ & 54 \\
\hline \multirow{2}{*}{$\mathrm{pH}$ buffer } & \multirow{2}{*}{$\begin{array}{l}\text { Aerobic batches and } \\
\text { columns }\end{array}$} & $\mathrm{Na}_{2} \mathrm{HPO}_{4}$ & 433 \\
\hline & & $\mathrm{NaH}_{2} \mathrm{PO}_{4}$ & 234 \\
\hline \multirow{12}{*}{ Trace elements } & \multirow{12}{*}{$\begin{array}{l}\text { Aerobic } \\
\text { batches and } \\
\text { columns }\end{array}$} & $\mathrm{FeCl}_{2} \cdot 4 \mathrm{H}_{2} \mathrm{O}$ & 1.2 \\
\hline & & $\mathrm{CoCl}_{2} \cdot 6 \mathrm{H}_{2} \mathrm{O}$ & 1.2 \\
\hline & & $\mathrm{MnCl}_{2} .4 \mathrm{H}_{2} \mathrm{O}$ & 0.3 \\
\hline & & $\mathrm{CuCl}_{2} \cdot 2 \mathrm{H}_{2} \mathrm{O}$ & 0.018 \\
\hline & & $\mathrm{ZnCl}_{2}$ & 0.03 \\
\hline & & $\mathrm{HBO}_{3}$ & 0.03 \\
\hline & & $\left(\mathrm{NH}_{4}\right) 6 \mathrm{Mo}_{7} \mathrm{O}_{24} \cdot 4 \mathrm{H}_{2} \mathrm{O}$ & 0.05 \\
\hline & & $\mathrm{Na}_{2} \mathrm{SeO}_{3} .5 \mathrm{H}_{2} \mathrm{O}$ & 0.06 \\
\hline & & $\mathrm{NiCl}_{2} \cdot 6 \mathrm{H}_{2} \mathrm{O}$ & 0.03 \\
\hline & & EDTA (tripex 2) & 0.6 \\
\hline & & $\mathrm{HCl} 36 \%$ & 0.0006 \\
\hline & & Resazurin & 0.3 \\
\hline \multirow{4}{*}{$\begin{array}{l}\text { Redox specific } \\
\text { compounds }\end{array}$} & Nitrate reducing columns & $\mathrm{NaNO}_{3}$ & 850 \\
\hline & \multirow{2}{*}{ Sulfate reducing columns } & $\mathrm{NaSO}_{4}$ & 1190 \\
\hline & & $\mathrm{Na}_{2} \mathrm{~S} .9 \mathrm{H}_{2} \mathrm{O}$ & 120 \\
\hline & Methanogenic columns & $\mathrm{Na}_{2} \mathrm{~S} .9 \mathrm{H}_{2} \mathrm{O}$ & 120 \\
\hline
\end{tabular}

Note: aerobic column medium was $60-80 \%$ oxygen saturated; other column media were flushed with $\mathrm{N}_{2}$ and did not contain oxygen.

Table S4.2 Abiotic under aerobic conditions within 6 weeks.

\begin{tabular}{lllllll}
\hline PhACs & MET & CAF & PRO & CBZ & NAP & IBP \\
\hline Removal (\%) & 29.0 & 27.1 & 55.0 & 22.4 & 14.8 & 24.7 \\
\hline
\end{tabular}




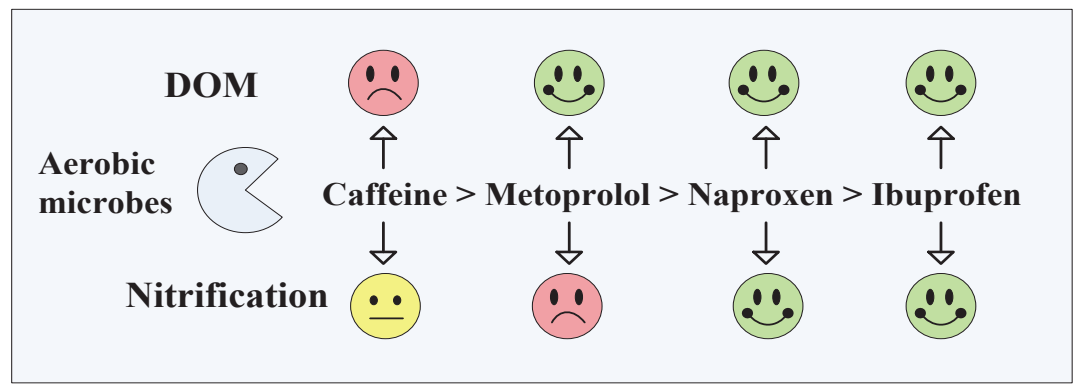

Constructed wetland 


\section{Chapter 5}

Effects of dissolved organic matter and nitrification on biodegradation of pharmaceuticals in aerobic enrichment cultures

A modified version of this chapter has been submitted as

He, Y., Langenhoff, A.A.M., Comans, R.N.J., Sutton, N.B., Rijnaarts, H.H.M. Effects of dissolved organic matter and nitrification on biodegradation of pharmaceuticals in aerobic enrichment cultures. Water Research. 


\begin{abstract}
Natural dissolved organic matter (DOM) and nitrification play an important role in biodegradation of pharmaceutically active compounds (PhACs) in aerobic zones of constructed wetlands (CWs). This study used an enrichment culture originating from $\mathrm{CW}$ sediment to study the effect of DOM and nitrification on aerobic biodegradation of PhACs. The enriched culture showed differences in biodegradation of seven PhACs with varying physicochemical properties. The culture degraded caffeine (CAF), metoprolol (MET), naproxen (NAP), and ibuprofen (IBP) with a consistent biodegradability order of CAF > MET > NAP > IBP. Biodegradation of propranolol, carbamazepine, and diclofenac was negligible in all cases. In the presence of DOM, CAF biodegradation was inhibited due to competition with the easily biodegradable DOM. Conversely, DOM enhanced biodegradation of MET, NAP, and IBP. We hypothesize that DOM can 1) sustain growth of more biomass capable of degrading PhACs, and/or 2) act as a structural analogue to stimulate the production of enzymes for PhAC biodegradation, and/or 3) act as electron shuttles that may support the metabolic or co-metabolic PhACs degradation. Finally, nitrification enhanced biodegradation of NAP and IBP and mineralization of the PhAC mixture as well as less biodegradable DOM, which may result from co-metabolism of ammonia oxidizing bacteria or enhanced heterotrophic microbial activity under nitrifying conditions. MET biodegradation was inhibited in the presence of nitrification. No influence of nitrification was observed for the biodegradation of the readily biodegradable CAF under nitrification conditions. DOM and nitrification effects on PhAC biodegradation in CWs gained from this study can be used in strategies to improve $\mathrm{CW}$ operation, namely: designing hydraulic retention times based on the biodegradability order of specific PhACs; applying DOM amendment; and introducing consistent ammonium streams to increase removal of PhACs of interest.
\end{abstract}

\title{
Keywords
}

Pharmaceuticals; Aerobic biodegradation; DOM; Nitrification; Enhancement; Inhibition 


\subsection{Introduction}

The occurrence and accumulation of pharmaceutically active compounds ( $\mathrm{PhACs}$ ) become a growing concern with the extensive detection of these contaminants in wastewater effluent, surface water, groundwater and sediment [165]. Some PhACs are beginning to be associated with adverse effects in aquatic organisms at environmentally relevant concentrations, i.e. ng/l to $\mu \mathrm{g} / 1$ [118]. The conventional wastewater treatment plants (WWTPs) could be a key barrier to eliminate $\mathrm{PhAC}$ discharge into the surface water, but are at this moment not designed to treat $\mathrm{PhACs}$ sufficiently [224]. Therefore, development of post-treatment processes in addition to conventional WWTPs are important ways to further remove PhACs from domestic effluents.

An alternative technique that could be coupled to WWTP is a constructed wetland (CW), which is sustainable, inexpensive in construction and easy to operate and maintain [272]. In recent years CWs have shown promising functionality for PhAC removal [400]. PhAC removal in CWs involves various mechanisms such as photodegradation, sorption, biodegradation, and phytoremediation [171]. Of those mechanisms, biodegradation plays a main role in the transformation and mineralization of PhACs [224]. PhAC biodegradation from wastewater is favored by aerobic conditions [400]. Oxygen supporting aerobic biodegradation of PhACs can be present in CWs throughout the water column in free water surface flow CWs, in the upper sediment layer, and in rhizosphere area of CWs [108]. Therefore, it is of importance to study the aerobic biodegradation of PhACs in CWs functioning as contaminant remediation systems. To date, aerobic biodegradation of PhACs in $\mathrm{CW}$ bioactive sediment or using enrichment cultures is hardly investigated, compared to related studies with soil and river sediment [204, 231, 406]. Therefore, CW sediment is the focus of this study.

CWs contain a large and potentially reactive pool of natural dissolved organic matter (DOM), and DOM play an important role in the bioavailability and fate of contaminants in CWs [74]. DOM is a complex mixture of organic compounds varying widely in molecular weight, from small molecules like organic acids to intermediate polymers like hemicellulose, and large humic substances such as humic acids (HA) and fulvic acids (FA) [195]. DOM is believed to interact with aerobic microbial communities influencing the biodegradation of organic contaminants through various mechanisms, including catabolic processes, redox cycling, and nutrient bioavailability [353]. For example, humic substances consisting of redox-mediating 
functional groups can significantly affect biodegradation of organic contaminants by acting as electron shuttles between electron donors and electron acceptors [125, 253]. The effect of DOM on the biodegradation of pesticides, herbicides, and polycyclic aromatic hydrocarbon are well investigated [353]. However, studies on the DOM impact on aerobic biodegradation of PhACs as emerging contaminants are limited.

Nitrification is an important redox reaction under aerobic conditions. Nitrification is known to enhance PhAC removal in various systems, e.g. nitrifying activated sludge (NAS), and membrane bioreactors $[28,90,346]$. It is believed that the ammonia monooxygenase (AMO) enzyme in ammonia oxidizing bacteria (AOB) can co-metabolize non-target PhACs in biochemical processes $[33,390]$. Nevertheless, the co-metabolism occurs for some PhACs, but is ineffective for others. For example, the biodegradation rate of $17 \alpha$-ethinylestradiol (EE2) significantly decreased after AOBs were inhibited, whereas trimethoprim biodegradation was not influenced by AOB inhibition in the same NAS systems [196]. Moreover, contradicting findings of nitrification on PhAC biodegradation were reported in different treatment systems. Fernandez-Fontaina et al. [121] showed that AOB enhanced biodegradation of ibuprofen (IBP) in NAS, whereas AOB was ineffective for IBP biodegradation in biofiltration columns packed with anthracite [301]. To date, there is no information available on the role of nitrification in biodegradation of PhACs in $\mathrm{CW}$ sediment microbial culture, especially for PhACs with different physicochemical properties.

This study applied a microbial enrichment culture originating from CWs to investigate the effect of DOM and nitrification on the aerobic biodegradation of a mixture of seven PhACs with different physicochemical properties. This research aims to elucidate the role of DOM and nitrification in CWs on PhAC biodegradation under aerobic conditions.

\subsection{Materials and methods}

\subsubsection{Chemicals and reagents}

PhACs were purchased commercially: $( \pm$ )-metoprolol (+)-tartrate salt (MET, $\geq 98 \%$ ), caffeine (CAF, $\geq 99 \%$ ), ( \pm )-propranolol hydrochloride (PRO, $\geq 99 \%$ ), CBZ ( $\geq 99 \%$ ), NAP ( $\geq 98 \%$ ), IBP $(\geq 98 \%$ ), and fenoprofen calcium salt (FEN, $\geq 97 \%$ ) from Sigma-Aldrich (USA). PhAC mixture stock $(20 \mathrm{mg} / \mathrm{L})$ was prepared with MilliQ water (Millipore, USA). Acetonitrile with $0.1 \%$ formic acid and water with $0.1 \%$ formic acid (Biosolve B.V., the Netherlands) were 
used for ultra-performance liquid chromatography (UPLC) analysis. All other chemicals used were of analytical grade.

\subsubsection{Batch experiments}

Batch experiments were performed to study the effect of DOM and nitrification on the aerobic biodegradation of a mixture of seven PhACs in a microbial enrichment culture. The enriched culture is originally from $\mathrm{CW}$ sediment microbial culture, which was acclimated aerobically with $\mathrm{PhAC}$ mixture $(1 \mathrm{mg} / \mathrm{l} \mathrm{each})$ in growth medium. The medium composition is the same as shown in Table S4.1, except the $\mathrm{NH}_{4} \mathrm{Cl}$ added in the medium was $157 \mathrm{mg} / \mathrm{l}$. The mixed sediment was collected from two CWs that are applied as post-treatment processes for two Dutch WWTPs. During the 22 months incubation, the inoculum was amended with PhAC mixture repeatedly. Afterwards, $10 \%$ of the enrichment was transferred to fresh aerobic medium spiked with PhAC mixture, and incubated for 2 weeks to obtain sediment free enrichment culture. After repeating the transfer twice, 10\% of the active PhAC degrading enrichment was used for our batch experiments. TOC analysis confirmed that no residual organic matter $(<0.3 \mathrm{mg} / \mathrm{l}$, detection limit) was transferred from the original sediment inoculum to our batch experiments.

Table 5.1 Designed experimental conditions to investigate the effect of DOM and nitrification on removal of PhACs.

\begin{tabular}{cccc}
\hline Conditions & $\begin{array}{c}\text { PhACs } \\
(1 \mathrm{mg} / \mathrm{l} \mathrm{each})\end{array}$ & $\begin{array}{c}\text { DOM } \\
(22.8 \mathrm{mg} / \mathrm{l} \text { in carbon })\end{array}$ & $\begin{array}{c}\text { Nitrification } \\
\text { (ammonium, 41.7 mg/l })\end{array}$ \\
\hline A & $\sqrt{ }$ & $\times$ & $\times$ \\
B & $\sqrt{ }$ & $\sqrt{ }$ & $\times$ \\
C & $\sqrt{ }$ & $\times$ & $\sqrt{ }$ \\
D & $\sqrt{ }$ & $\sqrt{ }$ & $\sqrt{ }$ \\
E & $\times$ & $\sqrt{ }$ & $\times$ \\
\hline
\end{tabular}

For the experiments, five batch conditions were designed to reach the research aims (Table 5.1). Nitrification was inhibited by spiking with allylthiourea (ATU, $5 \mathrm{mg} / \mathrm{l}$ ) in both conditions A and B. Condition E was only amended with DOM to determine its biodegradability in the microbial enrichment culture. For each condition, biotic batches were conducted in triplicate and abiotic controls in duplicate. Abiotic bottles were prepared by autoclaving for $20 \mathrm{~min}$ at $120{ }^{\circ} \mathrm{C}$ and adding chemical inhibitors $\left(0.3 \mathrm{~g} / \mathrm{L} \mathrm{HgCl}_{2}\right.$ and $1.3 \mathrm{~g} / \mathrm{L}$ 
$\mathrm{NaN}_{3}$ ) after cooling down. Milli-Q water was added to batches without DOM, ATU or chemical inhibitor addition to maintain the same volume and $\mathrm{PhAC}$ concentration in all batches.

Batch bottles with over-pressured air as gas phase were sealed with rubber stoppers. Batch bottles were incubated on a shaker $(120 \mathrm{rpm})$ at $20^{\circ} \mathrm{C}$ and covered with aluminum foil to avoid photodegradation of PhACs. The batch experiments lasted for 35 days and liquid samples were withdrawn at day $0,1,2,4,7,10,14,22,28$, and 35. Samples were pre-treated by centrifugation at $10000 \mathrm{rpm}$ for $5 \mathrm{~min}$ prior to UPLC and ion chromatography (IC) analyses. DOM samples were taken at day 0 and 35 from triplicate batches and mixed to compare the composition variation during the biodegradation. $50 \mathrm{ml}$ liquid samples were filtered using $0.2 \mu \mathrm{m}$ Isopore ${ }^{\mathrm{TM}}$ membrane filters (Milipore, Ireland) and stored in centrifuge tubes at $-20^{\circ} \mathrm{C}$ before DNA extraction. At day 23 ATU was re-spiked to maintain the nitrification inhibition and gas phase was exchanged to maintain the $\mathrm{O}_{2}$ supply.

\subsubsection{DOM extraction, fractionation and characterization}

DOM was extracted from the sediment together with litters in the $\mathrm{CW}$ to obtain natural organic matter. The sediment was mixed with Milli Q water (1:1, wet w/w) by shaking at 180 rpm for $1 \mathrm{~h}$. The mixture was centrifuged at $3750 \mathrm{rpm}$ for $15 \mathrm{~min}$ and the supernatant obtained was further centrifuged at $10000 \mathrm{rpm}$ for $20 \mathrm{~min}$. Afterwards the supernatant was filtered through $0.45 \mu \mathrm{m}$ mixed cellulose ester filters (ME25/21, Whatman, USA) to collect the DOM. DOM samples collected at day 0 and 35 were fractionated according to the procedure reported by Van Zomeren and Comans [354] to get the fractions including HA, FA, hydrophilic compounds (Hy), and hydrophobic neutrals (HON). Work-flow of the procedure is summarized in Figure S5.1. Recovery of the fractionation procedure was $84-96 \%$ in this work. For characterization, the DOM samples were first freeze-dried to solid material by an alpha 2-4 LD+ freeze dryer (Martin Christ GmbH, Germany) and then characterized by using a Bruker Tensor 27 Fourier transform infrared spectroscopy (FTIR) equipped with a Platinum ATR accessory (Bruker, Germany).

\subsubsection{Chemical analysis}

Concentrations of PhACs were measured by a UPLC (Ultimate 3000, Thermo, USA) equipped with diode array detection, as previously described [156]. Quantification of PhACs was performed based on the internal standard (FEN) and external calibration standards. 
Concentrations of $\mathrm{NO}_{2}{ }^{-}$and $\mathrm{NO}_{3}{ }^{-}$were analyzed by an IC (Dionex ICS-2100, Thermo, USA) as described in De Wilt et al. [375]. Concentrations of $\mathrm{O}_{2}$ and $\mathrm{CO}_{2}$ were followed by a gas chromatography (GC-2010, Shimadzu, Japan) according to the method described in in De Wilt et al. [375]. A Sievers ${ }^{\mathrm{TM}} 900$ Series TOC Analyzer (GE Analytical Instrument, USA) was used to quantify the concentrations of DOM and its fractions.

\subsubsection{Molecular analysis}

DNA was extracted from the filtered samples using PowerSoil ${ }^{\circledR}$ DNA Isolation Kits (MoBio Laboratories, USA) according to the manufacturer's protocol. Concentration and purity of the extracted DNA were estimated by a Nanodrop spectrophotometer (Denovix, DS-11, USA). The DNA extracts were stored at $-20{ }^{\circ} \mathrm{C}$ until further analysis. Quantitative PCR (qPCR) was used to quantify the abundances of bacterial 16S rRNA gene and AOB amoA genes. The qPCR measurements were performed in triplicate by a CFX384 touch real-time PCR detection system (Bio-Rad, Veenendaal, the Netherlands). In the reaction mixture, $2 \mu 1$ diluted sample (10 times dilution) was mixed with $2 \mu$ master mix comprising of $\mathrm{iQ}^{\mathrm{TM}}$ SYBR ${ }^{\circledR}$ Green (Bio-Rad), primers (forward and reverse), and DNase-free water (50:2:2:26). Standards and blanks were prepared similarly to the samples. Standard curves were obtained by using serial dilutions of a known amount of plasmid DNA containing a fragment of the respective genes. Detailed information of the primers and cycling conditions used for qPCR analyses are summarized in Table S5.1. Abundance of amoA was represented in absolute concentration and 16S-rRNA normalized values.

\subsection{Results and discussion}

\subsubsection{Biodegradation of PhACs}

Biodegradation of the seven PhACs tested showed remarkable differences. For example, in condition $\mathrm{C}$ with nitrification present (the same as during enrichment for 23 months), CAF, MET, NAP, and IBP were completely removed within 35 days (Figure 5.1). This can be ascribed to biodegradation as no obvious removal was found in abiotic controls (Figure S5.2). Our study obtained comparable or higher removal for the biodegradation of CAF, MET, and NAP than biodegradation observed in microbial cultures of soil and river sediments [204, 231, 406]. A CW sediment-based research presented that $50 \%$ of IBP can be removed within nine days resulting from sorption onto sediment and biodegradation by natural microbial population [81]. In comparison, our sediment-free batches gave a 50\% biodegradation 
approximately within 22 days. Similar with our findings (Figure 5.1), PRO, DFC, and CBZ were reported to be persistent during biodegradation tests [345, 384, 408]. The same conclusions can be drawn for the biodegradability of the seven PhACs in conditions A (nitrification inhibition), B (nitrification inhibition and DOM addition), and D (with both DOM and nitrification present), as indicated in Figure S5.3. Especially, in conditions B and D with DOM addition, no obvious sorption of PhACs onto the extracted DOM was found in the abiotic controls (Figure S5.2). PRO, DFC, and CBZ will not be discussed in the following content considering their negligible biodegradation.

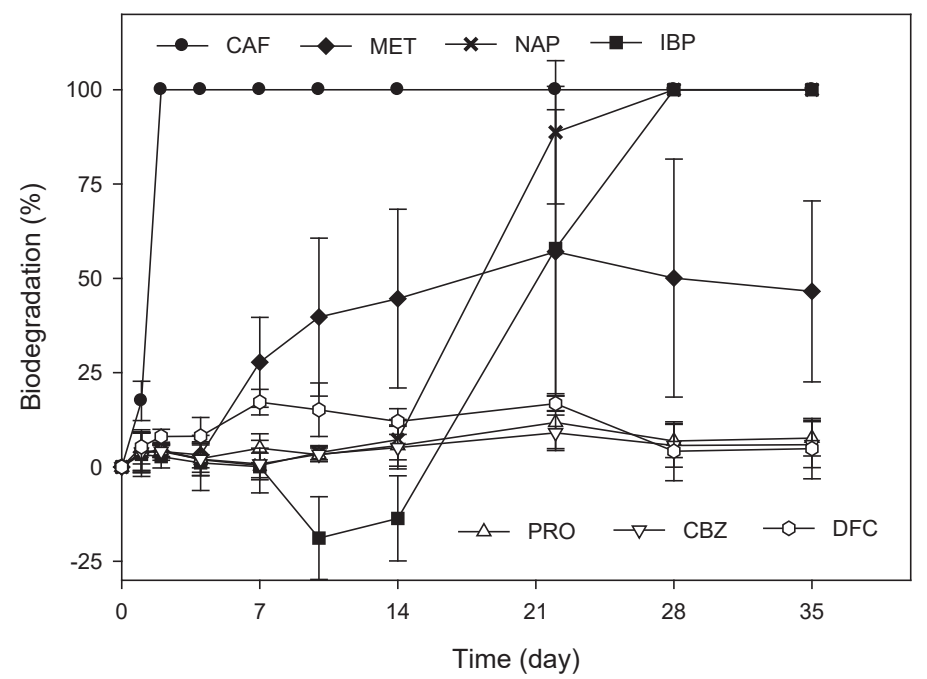

Figure 5.1 Biodegradation of a mixture of seven PhACs in the microbial enrichment culture in the condition $\mathrm{C}$ with nitrification present. Results are mean values \pm standard deviation $(\mathrm{n}=3)$.

Microorganisms seem to have a preferential order when biodegrading $\mathrm{PhACs}$, i.e. less biodegradable PhACs were only biodegraded once the readily degradable $\mathrm{PhACs}$ were removed. As shown in Figure 5.1, CAF was first biodegraded during day 0-2 followed by MET during day 2-14, and finally NAP and IBP during day 14-22. The notable standard deviation of MET biodegradation was due to different biodegradation capacity in our triplicate batches, but the biodegradation tendency in the triplicates are consistent where MET was not further biodegraded after day 14 (Figure S5.3). The microbial biodegradation preference was also observed for the other conditions: CAF > MET > NAP > IBP (Figure S5.4). Therefore, our study demonstrates a clear microbial preference in the order of PhACs biodegraded regardless of conditions with or without DOM and nitrification. 
PhACs can be the single carbon substrate to support microbial growth, as seen in condition A. Looking at the microbial respiration, $\mathrm{O}_{2}$ was consumed and $\mathrm{CO}_{2}$ was produced from $\mathrm{PhACs}$ in condition A (Figure 5.2). Moreover, the amount of total bacteria in this condition increased from $1 \times 10^{4}$ to $4.7 \times 10^{4}$ copies/ml after 35 days incubation (Figure S5.5). Both the microbial respiration and growth supported by $\mathrm{PhACs}$ indicate that PhACs can be used as single carbon substrate, and results in a selective pressure to enrich microorganisms capable of biodegrading PhACs. Similarly, ketoprofen was reported to act as a single source of carbon and energy for microorganisms in a membrane bioreactor [298]. These findings demonstrate the possibility to enrich microorganisms capable of biodegrading PhACs for the use of enhanced biodegradation technologies such as bioaugmentation. Iasur-Kruh et al. [181] successfully enriched and isolated a pure culture of Novosphingobium JEM-1 from CW sediment and bioaugmented the culture to $\mathrm{CW}$ sediment film to achieve $40 \%$ removal of estron (E1) and 17-estradiol (E2) in batch experiments.

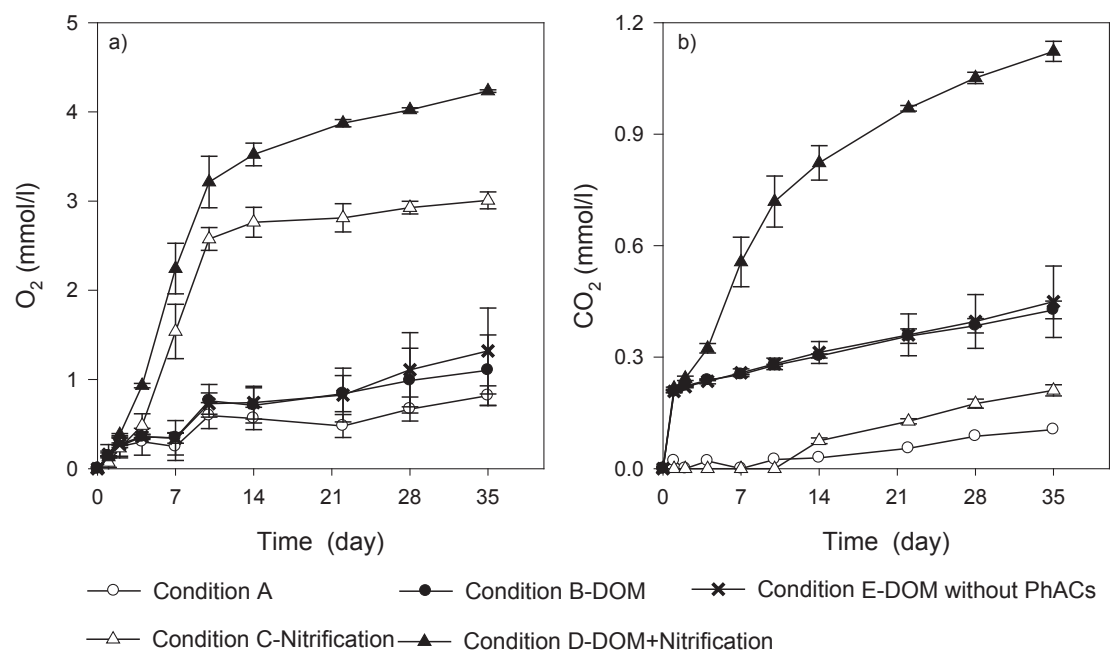

Figure 5.2 Microbial respiration in the five conditions: a) consumption of $\mathrm{O}_{2}$ and b) production of $\mathrm{CO}_{2}$. Results are mean values \pm standard deviation $(\mathrm{n}=3)$.

\subsubsection{Effect of DOM on PhAC biodegradation}

First, the characteristics of the DOM extracted from CW sediment were investigated. The DOM fractions comprised 5\% HA, 35\% FA, 27\% Hy, and 33\% HON (Figure 5.3, condition E with only DOM present). As presented in the FTIR spectra (Figure S5.6), the main absorption bands of the extracted DOM corresponded to the following functional groups: 1) 
$3390 \mathrm{~cm}^{-1}$ (O-H stretching of inter- and intra- molecular hydrogen bonds); 2) $1639 \mathrm{~cm}^{-1}$ (aromatic $\mathrm{C}=\mathrm{C}$ skeletal vibrations, asymmetric stretching of $\mathrm{C}=\mathrm{O}$, symmetric stretching of COO-); 3) $1359 \mathrm{~cm}^{-1}$ (C-O asymmetric stretching of COOH conditions); 4) $1105 \mathrm{~cm}^{-1}$ (C-O stretching of alcohols and carbohydrate) [63, 347].

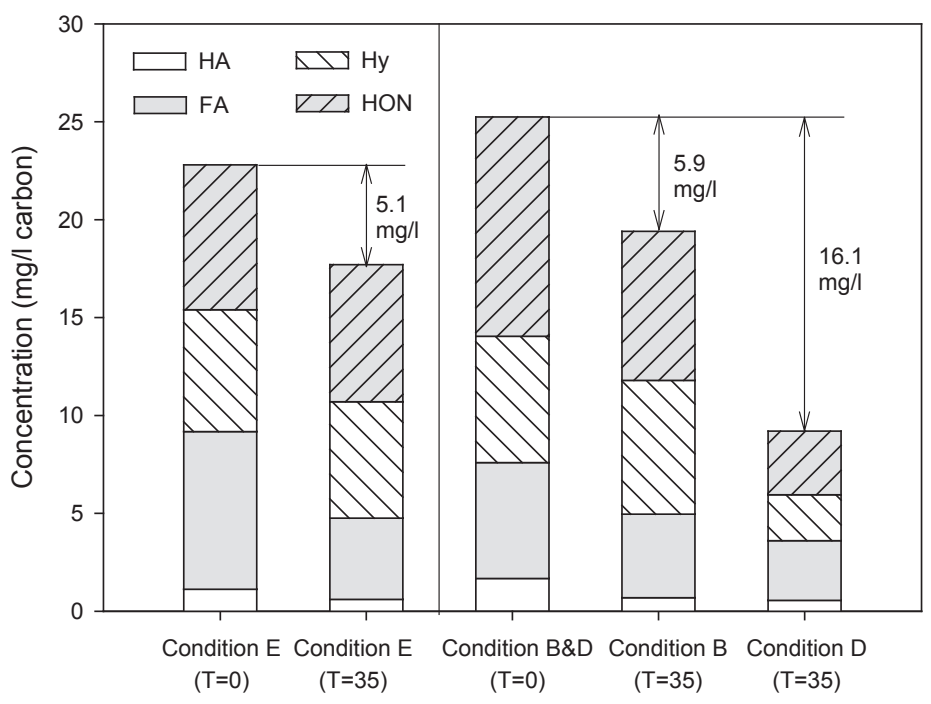

Figure 5.3 Composition of DOM fractions at day 0 and 35 in conditions with DOM present $(\mathrm{HA}=$ humic acid, FA= fulvic acid, Hy = hydrophilic compounds, $\mathrm{HON}$ = hydrophobic neutrals).

Second, the biodegradability of the DOM was evaluated by microbial respiration and fractionation studies. DOM was rapidly mineralized to $\mathrm{CO}_{2}$ in the first two days followed by a relatively slow mineralization rate (Figure $5.2 \mathrm{~b}$, condition E). A similar rapid $\mathrm{CO}_{2}$ production was also found in conditions B and D with DOM present. Furthermore, we confirmed that the significant $\mathrm{CO}_{2}$ production in the first two days was related to DOM mineralization rather than DOM breakdown due to abiotic process (results not shown). The observed fast production of $\mathrm{CO}_{2}$ may indicate that the microbial enrichment culture first rapidly mineralized DOM which are relatively easily biodegradable, followed by mineralization of the less biodegradable DOM with a slower rate. This microbial preference towards carbon substrate was reported in previous work, where the authors found that Gramnegative bacteria in the soil prefer to use fresh plant-derived carbon while Gram-positive bacteria use more older soil organic matter-derived carbon independent on the soil depth [207]. The microbial biodegradation preference for DOM compositions is comparable with the microbial preference found among the PhACs. 
In terms of the microbial respiration, the easily biodegradable DOM contributes to half of the $\mathrm{CO}_{2}$ production and the less biodegradable DOM is responsible for the other half. In total $22 \%$ DOM was consumed during 35 days incubation, with HA and FA being the main metabolized DOM fractions (Figure 5.3, respectively $46 \%$ and $49 \%$ consumed). This is in line with a previous modelling study, where HA and FA are mentioned as significant DOM fractions for microbial respiration [335]. The functional groups of 3390 and $1639 \mathrm{~cm}^{-1}$ in DOM disappeared after 35 days biodegradation (Figure S5.6), which indicates that the microbial enrichment culture is capable to transform DOM with those structures.

\subsubsection{DOM inhibits biodegradation of PhACs}

The effect of DOM on PhAC biodegradation was identified by comparing the biodegradation efficiencies of PhACs between conditions A and B as well as between conditions $\mathrm{C}$ and $\mathrm{D}$. In the presence of DOM, CAF biodegradation was delayed. CAF in conditions $\mathrm{A}$ and $\mathrm{C}$ without DOM addition was completely biodegraded within 2 days. This coincides with the time to consume the easily biodegradable DOM (Figure 5.4). Furthermore, CAF contains functional groups of asymmetric stretching of $\mathrm{C}=\mathrm{O}\left(1639 \mathrm{~cm}^{-1}\right)$ [150]. These functional groups are also present in our extracted DOM and they disappeared during 35 days of incubation, most likely due to biodegradation (Figure S5.6). Therefore, we assume that the easily biodegradable DOM competed with the readily biodegradable CAF resulting in a delay in CAF biodegradation till day 4 in the presence of DOM (Figure 5.4, conditions B and D). The competition between the easily biodegradable DOM and CAF also indicates a microbial biodegradation preference, which is comparable with the preferences found among PhACs and among DOM compositions. 


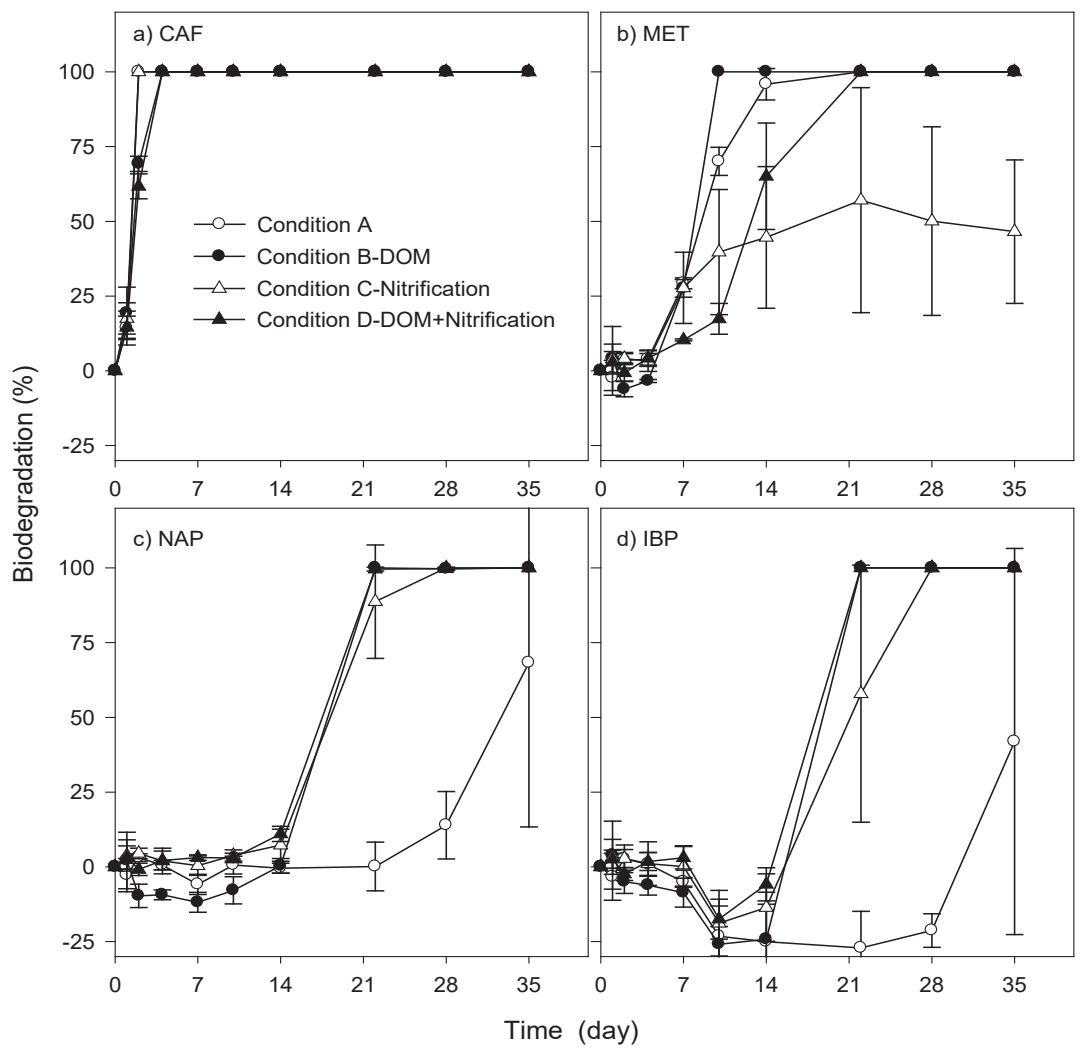

Figure 5.4 Biodegradation of the four biodegradable PhACs in four experimental conditions: a) caffeine, b) metoprolol, c) naproxen, d) ibuprofen. Results are mean values \pm standard deviation $(\mathrm{n}=$ $3)$.

\subsubsection{DOM enhances biodegradation of PhACs}

As opposed to CAF, biodegradation of MET, NAP, and IBP was enhanced by DOM addition. After the easily biodegradable DOM and the readily biodegradable CAF was utilized, microorganisms started degrading the other PhACs. A faster biodegradation of MET, NAP, and IBP was found in conditions B and D compared to conditions A and C (Figure 5.4). As with MET in condition $\mathrm{C}$, the standard deviation of IBP biodegradation at day 22 was caused by different biodegradation capacity in triplicate batches (Figure S5.7), but in general the IBP biodegradation in this condition was slower than in the group D (Figure 5.4d).

There appears to be various hypotheses to explain this DOM enhanced PhAC biodegradation. First, more biomass was found in the conditions with DOM addition (Figure S5.5, B > A, D > 
C). Furthermore, the extracted DOM can act as a co-substrate for the microbial enrichment culture as indicated by the increased $\mathrm{CO}_{2}$ production in conditions $\mathrm{B}$ and $\mathrm{D}$ compared to conditions $\mathrm{A}$ and $\mathrm{C}$ (Figure 5.2b). The observed higher biomass and higher microbial respiration with DOM addition indicate more active microbial community which may have increased PhAC biodegradation capacity. Rauch-Williams et al. [302] hypothesized that removal of organic contaminants including NAP and IBP was caused by a specialized microbial community growing on hydrophobic acids, i.e. HA and FA, as in our case.

Second, DOM may act as structural analogues to enhance PhAC biodegradation. By biodegrading DOM with similar molecular structures to PhACs, microorganisms may activate more enzymes that can readily biodegrade PhACs with similar structures. This structural analogue theory has been applied for the biodegradation of organic compounds such as pesticides, or cyclic ethers $[129,198]$.

Last, DOM can act as electron shuttles to stimulate microorganisms to degrade PhACs. DOM is a complex mixture of organics with many functional groups that could be potentially functional in redox reactions [353]. For example, quinones in DOM (especially in HA) can act as redox-mediating functional groups to stimulate the electron transfer between electron donors and electron acceptors and thus enhance redox reactions [253]. It has been confirmed that biodegradation of certain PhACs are dependent on the availability and concentrations of DOM [229, 249]. However, there are no studies that explicitly describe the mechanism of DOM in the enhancement of PhAC biodegradation. Further research efforts need be dedicated to understand the underlying mechanisms of these processes.

\subsubsection{Effect of nitrification on PhAC biodegradation}

Nitrification occurred in conditions $\mathrm{C}$ and $\mathrm{D}$ whereas it was inhibited in conditions $\mathrm{A}$ and $\mathrm{B}$ by the addition of ATU. $70 \%$ of the ammonium in batches was converted to $\mathrm{NO}_{3}^{-}$in conditions $\mathrm{C}$ and $\mathrm{D}$ along with biodegradation of PhACs. $\mathrm{No} \mathrm{NO}_{3}{ }^{-}$was formed in the other two conditions (Figure S5.8). In the q-PCR tests, amoA concentration was higher in conditions $\mathrm{C}$ and $\mathrm{D}$ as compared to the other two conditions, indicating an enrichment of AOBs (Figure S5.9). The absolute concentrations of amoA in conditions B and D might be influenced by humic acid presence (Figure S5.9), as reported by Schriewer et al. [319]. However, this effect in those two conditions was absent after normalization for abundance relative to the 16S rRNA gene abundance (Figure S5.9). In our nitrification conditions, nitrification plays an important role in microbial respiration as it stoichiometrically accounts 
for $39 \%$ and $38 \%$ of the $\mathrm{O}_{2}$ consumption for $\mathrm{NO}_{2}{ }^{-}$and $\mathrm{NO}_{3}{ }^{-}$production in conditions $\mathrm{C}$ and $\mathrm{D}$, respectively.

\subsubsection{Nitrification enhances biodegradation of $\mathrm{PhACs}$}

The effect of nitrification on PhAC biodegradation is seen by comparing the biodegradation efficiencies between conditions $\mathrm{A}$ and $\mathrm{C}$ and between conditions $\mathrm{B}$ and D. Nitrification enhanced the biodegradation of NAP and IBP. Significantly higher biodegradation of NAP and IBP was found in condition $\mathrm{C}$ than in condition A (Figure 5.4). No difference was observed between conditions B and D.

The enhancement by nitrification is likely a result of AOB driven co-metabolism. As demonstrated by Fernandez-Fontaina et al. [121], biodegradation of NAP and IBP was enhanced by $\mathrm{AOB}$ instead of contribution from heterotrophic microorganisms. AOB containing AMO can easily hydrolyze linear alkyl carbons especially for the secondary and tertiary carbons in the chain, due to its low specificity and broad substrate spectrum [121]. Such structures can be found in NAP and IBP (Figure 5.5, circles). Co-metabolic function of $\mathrm{AOB}$ was reported for many other PhACs in nitrifying bioreactors and membrane bioreactors $[310,390]$. It has been demonstrated that sludge-based wastewater treatment processes with significant nitrification showed higher removal efficiencies than those without nitrification for $90 \%$ of the investigated PhACs [384]. Comparable with sludge-based microbial cultures, our findings demonstrate positive effect of nitrification on PhAC biodegradation also in the $\mathrm{CW}$ sediment derived microbial cultures.

In our case, nitrification not only enhanced biodegradation of NAP and IBP but also enhanced mineralization of PhACs, as illustrated in the increased $\mathrm{CO}_{2}$ production in condition C compared to condition A (Figure 5.2b). In addition to increase $\mathrm{PhAC}$ mineralization, nitrification also stimulated mineralization of less biodegradable DOM. DOM fractionation reveals that condition D with nitrification showed more biodegradation of HA, Hy, and HON, compared to conditions B (Figure 5.3). The more biodegraded DOM in condition $\mathrm{D}$ was mainly mineralized to $\mathrm{CO}_{2}$, as illustrated by comparing the carbon balances between conditions $\mathrm{D}$ and $\mathrm{B}$. In condition $\mathrm{D}$, more DOM was consumed than in condition $\mathrm{B}$ (equal to $0.85 \mathrm{mmol} / 1 \mathrm{CO}_{2}$, Figure 5.3) and more $\mathrm{CO}_{2}$ was produced $(0.70 \pm 0.04 \mathrm{mmol} / \mathrm{l}$, Figure 5.2b). The enhanced biodegradation of less biodegradable DOM might be due to cometabolism via $\mathrm{AOB}$ and/or enhanced heterotrophic microbial activity. Microbial $\mathrm{CO}_{2}$ production was higher in conditions $\mathrm{C}$ and $\mathrm{D}$ with nitrification than in conditions $\mathrm{A}$ and $\mathrm{B}$, 
respectively (Figure 5.2b), which indicates higher heterotrophic microbial activity under nitrifying conditions. The higher heterotrophic microbial activity might contribute to the higher mineralization of $\mathrm{PhACs}$ and less biodegradable DOM under nitrifying conditions.

\subsubsection{Nitrification inhibits biodegradation of PhACs}

MET biodegradation was inhibited in the presence of nitrification, as indicated in Fig 4. To date, there is no literature referring to inhibition of $\mathrm{PhAC}$ degradation in nitrifying cultures; only enhanced PhAC biodegradation has been reported, as described at 3.3.1, or no effect on PhAC biodegradation has been observed [196]. To confirm the unexpected finding, we repeated our experiment with MET and the same conclusion was obtained (Table S5.2). The inhibitory effect of nitrification on MET is not yet well understood. Further research is needed to elucidate the observed discrepancies of nitrification effect on NAP and IBP, and MET.

Compared to the other three biodegradable PhACs, biodegradation of CAF was not obviously influenced by nitrification. The reason might be that CAF was readily biodegradable or the nitrification effect was not detected due to the designed sampling frequency.

\subsubsection{Implications for theory and practice}

In summary, the effect of DOM and nitrification on the biodegradation of PhACs is shown in Figure 5.5. Translating our findings to practice, first the clear biodegradability order among $\mathrm{PhACs}$ can provide an operational strategy for designing hydraulic retention time (HRT) for removing specific PhACs. Second, DOM addition could be a strategy to enhance the aerobic biodegradation of less biodegradable PhACs in CWs. Instead of harvesting dead plants seasonally and transporting them for special disposal, dead plant biomass can be (partially) disposed directly into a CW to obtain more natural DOM. Third, nitrification is beneficial for biodegradation of PhACs with certain structures. However, ammonium concentration is normally low in the $\mathrm{CW}$ influent, resulting in limited nitrification. A sub-stream of wastewater influent of WWTPs rich in ammonium could be introduced to CWs to strengthen the biodegradation of PhACs of interests. 


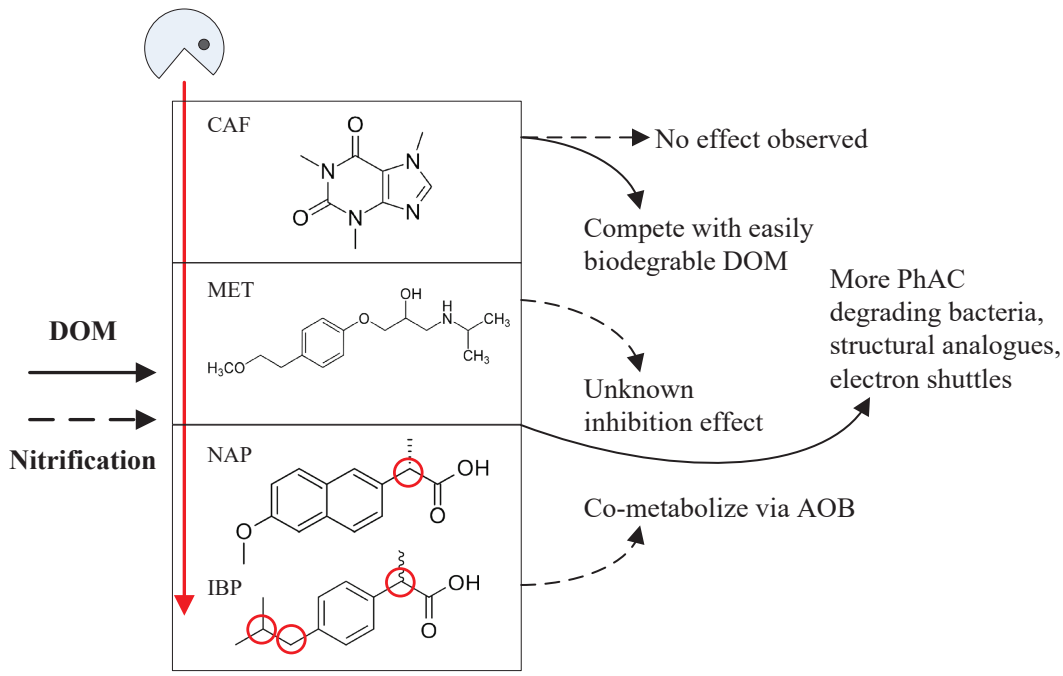

Figure 5.5 Proposed mechanisms of the effect of DOM and nitrification on biodegradation of caffeine, metoprolol, naproxen, and ibuprofen. DOM pathway represented in solid lines; nitrification pathways represented in dash lines. Directions of arrows show enhancement (up), inhibition (down), and no effect (horizontal). The red line represents the biodegradability order of the microbial enrichment culture. Red circles on the structure of NAP and IBP label the secondary and tertiary carbons in linear alkyl chains for hydroxylation by ammonia oxidizing bacteria (AOB).

\subsection{Conclusions}

This study applied a microbial enrichment culture originating from CW sediment to test the effect of DOM and nitrification on the biodegradation of PhACs in CWs. Our enrichment culture showed different biodegradation capacities for the seven PhACs that all had different physicochemical properties. CAF, MET, NAP, and IBP were completely biodegraded in most experimental conditions while biodegradation of PRO, CBZ, and DFC was negligible. In addition, aerobic microbial communities appear to have a biodegradation preference in the order of CAF $>$ MET $>$ NAP $>$ IBP. PhACs can be the sole carbon substrate to support microbial growth and respiration. This demonstrates the possibility to use PhACs as the single carbon substrate as a selective pressure to enrich microorganisms capable of biodegrading $\mathrm{PhACs}$ for the use of enhanced biodegradation technologies.

With DOM present, the easily biodegradable DOM was rapidly consumed followed by less biodegradable DOM. In general, the consumed DOM fractions are HA and FA, and the consumed DOM composition are with functional groups including $\mathrm{O}-\mathrm{H}$ stretching of inter- 
and intra- molecular hydrogen bonds, aromatic $\mathrm{C}=\mathrm{C}$ skeletal vibrations, asymmetric stretching of $\mathrm{C}=\mathrm{O}$, and symmetric stretching of COO-. Biodegradation of CAF competed with easily biodegradable DOM during the first two days. In contrast, DOM enhanced the biodegradation of MET, NAP, and IBP, potentially by either contributing more biomass capable of degrading PhACs, acting as structural analogues, or acting as electron shuttles to stimulate microorganisms to metabolize or co-metabolize PhACs.

Nitrification enhanced the biodegradation of NAP and IBP which may result from AOB driven co-metabolism. Additionally, nitrification enhanced mineralization of both $\mathrm{PhACs}$ and DOM fractions (Hy, HA, HON), indicating that heterotrophic microorganisms may flourish under nitrifying conditions. MET biodegradation was inhibited in the presence of nitrification. In comparison, no difference was observed for the readily biodegradable CAF in the presence or absence of nitrification.

This study contributes to the understanding of the impact of DOM on aerobic biodegradation of PhACs and the role of nitrification in PhAC biodegradation in enrichment cultures originating from $\mathrm{CW}$ sediment. For application, the clear biodegradability preference found among PhACs can offer a strategy to design different HRTs to remove corresponding PhACs. From the operational perspective, DOM additions and introducing consistent ammonium streams may be used to increase the potential of CWs to achieve higher PhAC biodegradation.

\section{Acknowledgement}

Authors thank Gerlinde Vink for the technical supervision of DOM fractionation. The support provided by China Scholarship Council (CSC) for the research of Yujie He at Wageningen University is kindly acknowledged. 


\section{Supplementary materials}

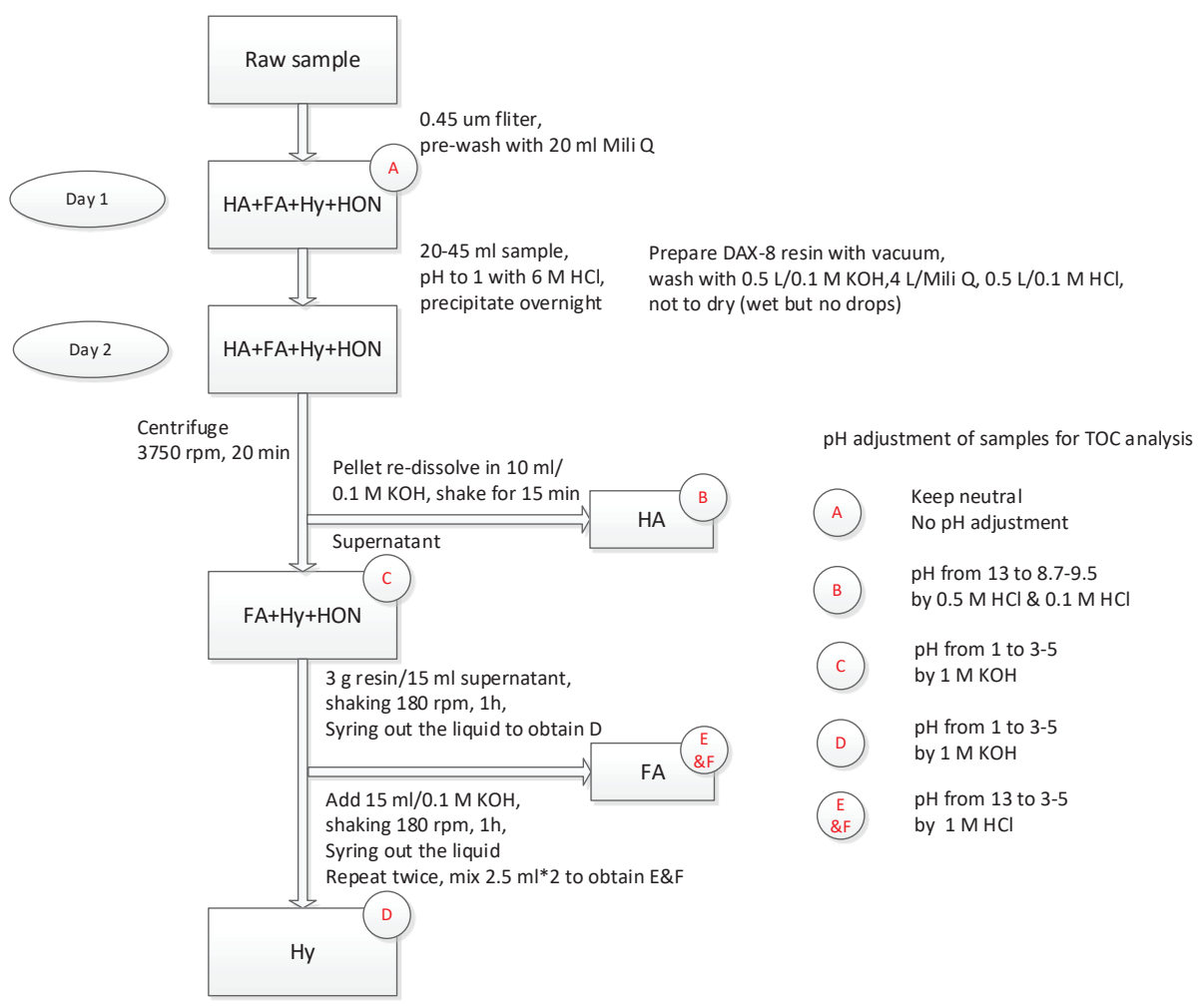

Figure S5.1 Workflow of DOM fractionation of humic acid (HA), fulvic acid (FA), hydrophilic compounds (Hy), and hydrophobicneutrals (HON). 

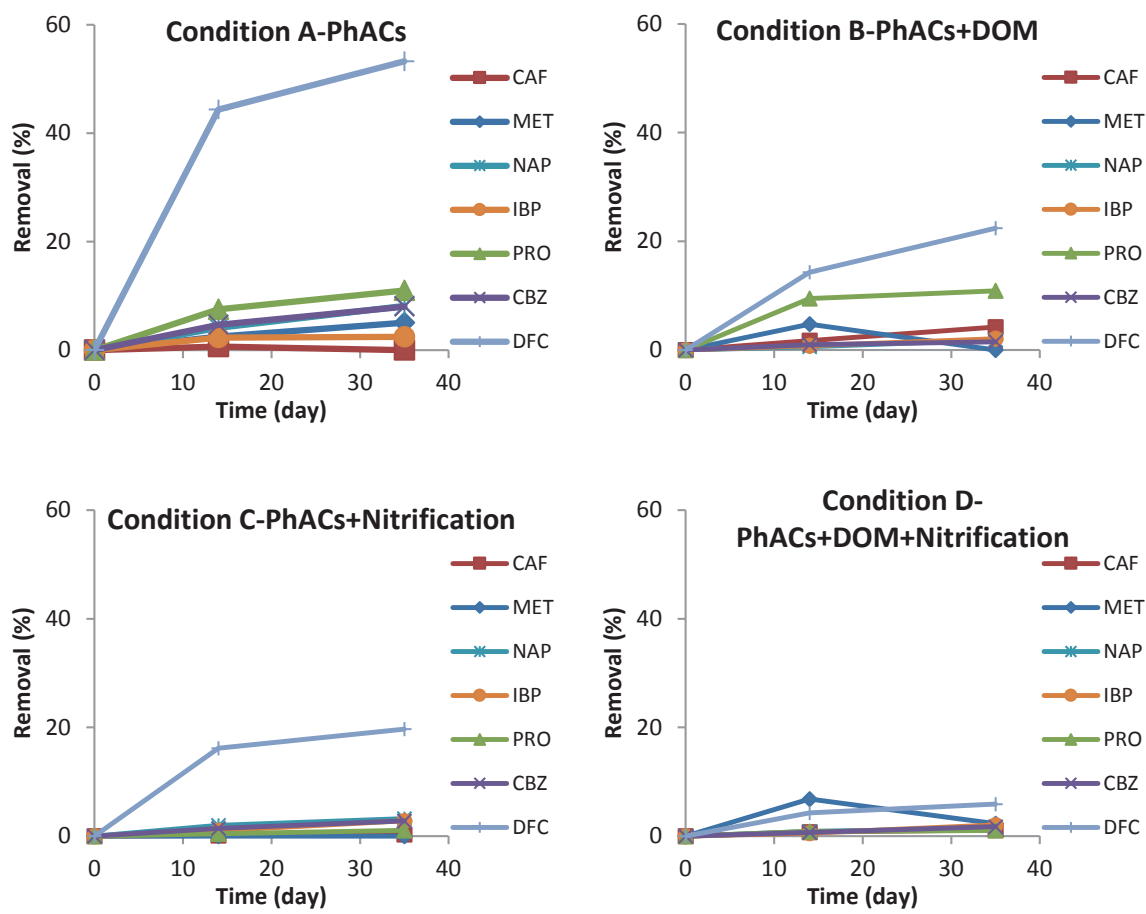

Figure S5.2 Abiotic removal of PhACs in four experimental conditions. Data are the mean value of duplicate results.

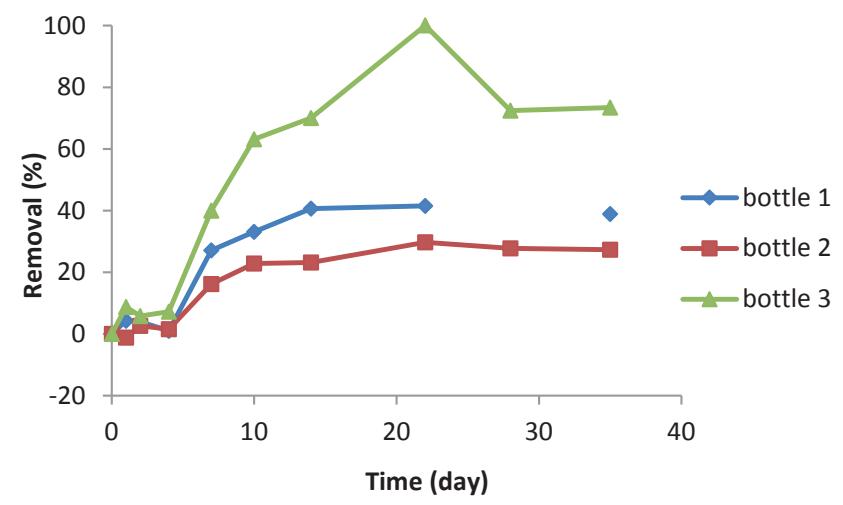

Figure S5.3 Biodegradation of metoprolol in triplicate bottles of condition C. Sample of bottle 2 at day 28 was missing due to operational mistake. 

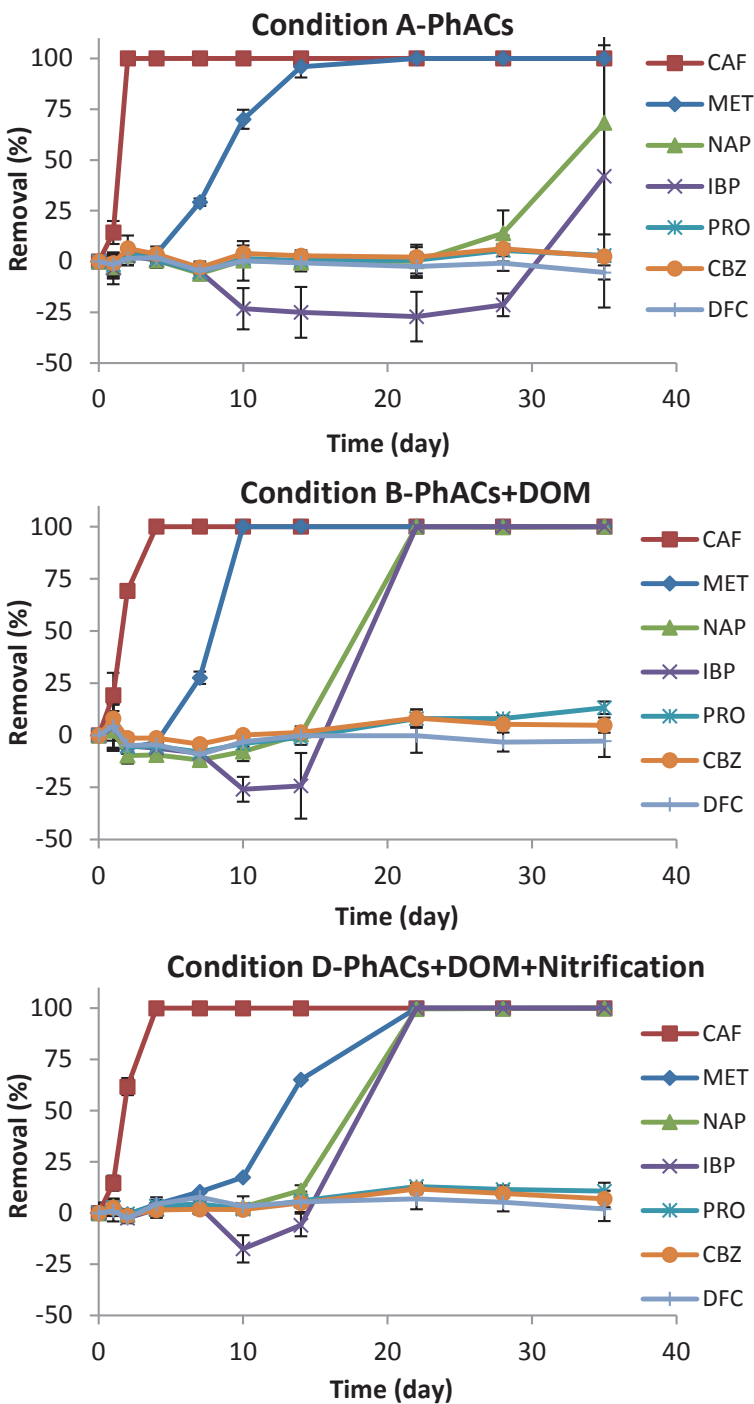

Figure S5.4 Biodegradation of $7 \mathrm{PhACs}$ in conditions A, B, and D. Results are mean values \pm standard deviation $(\mathrm{n}=3)$. The high standard deviation in the condition A at day 35 for NAP and IBP are due to the different biodegradation capacity in duplicate bottles. The biodegradability orders of PhACs are: CAF (day 0-2) > MET (day 2-22) > (NAP $>$ IBP) (day 22-35) in condition A; CAF (day 04) $>$ MET $($ day 4-10) $>(N A P>I B P)($ day 10-22) in condition B; CAF (day 0-4) $>($ MET $>N A P>I B P)$ (day 4-22) in condition D. 


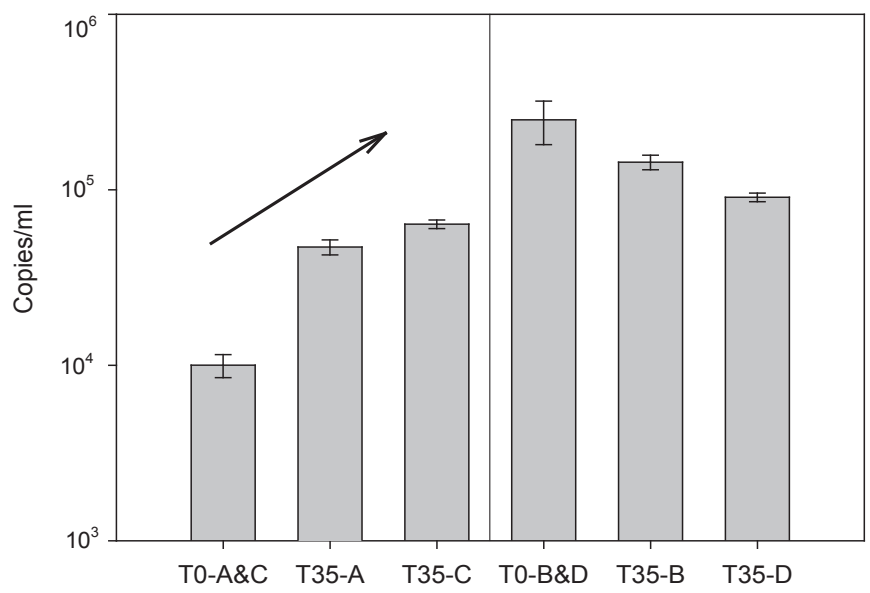

Figure S5.5 Abundance of total bacteria at day 0 and 35. Microbial source of conditions A and C are the enriched microbial culture; microorganism source of conditions B and D are the enriched microbial culture and microorganisms from the DOM. Results are mean values \pm standard deviation $(\mathrm{n}=3)$.

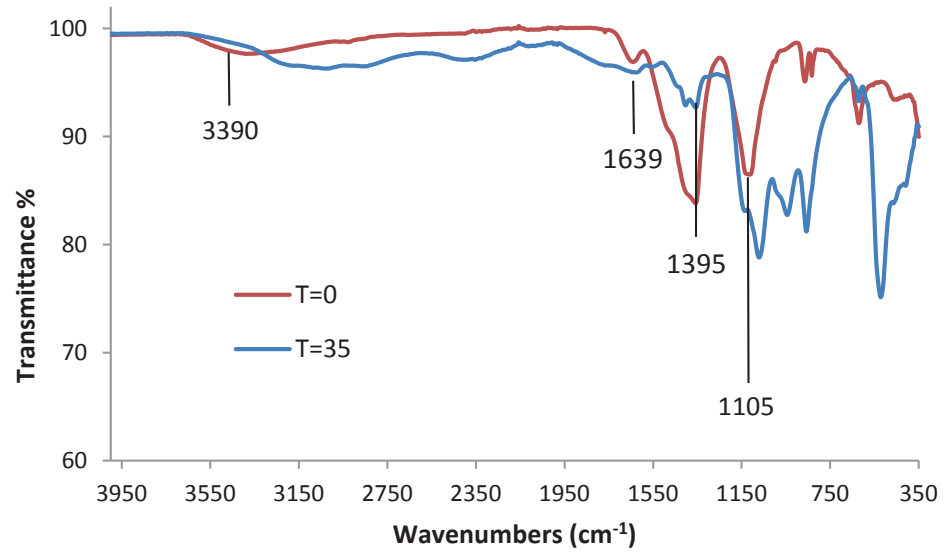

Figure S5.6 FTIR spectra of DOM at day 0 and 35. Absorption bands correspond to different functional groups: 1) $3390 \mathrm{~cm}^{-1}$ (O-H stretching of inter- and intra- molecular hydrogen bonds); 2) $1639 \mathrm{~cm}^{-1}$ (aromatic $\mathrm{C}=\mathrm{C}$ skeletal vibrations, asymmetric stretching of $\mathrm{C}=\mathrm{O}$, symmetric stretching of COO-); 3) $1359 \mathrm{~cm}^{-1}$ (C-O asymmetric stretching of COOH groups); 4) $1105 \mathrm{~cm}^{-1}$ (C-O stretching of alcohols and carbohydrate). 
Aerobic biodegradation

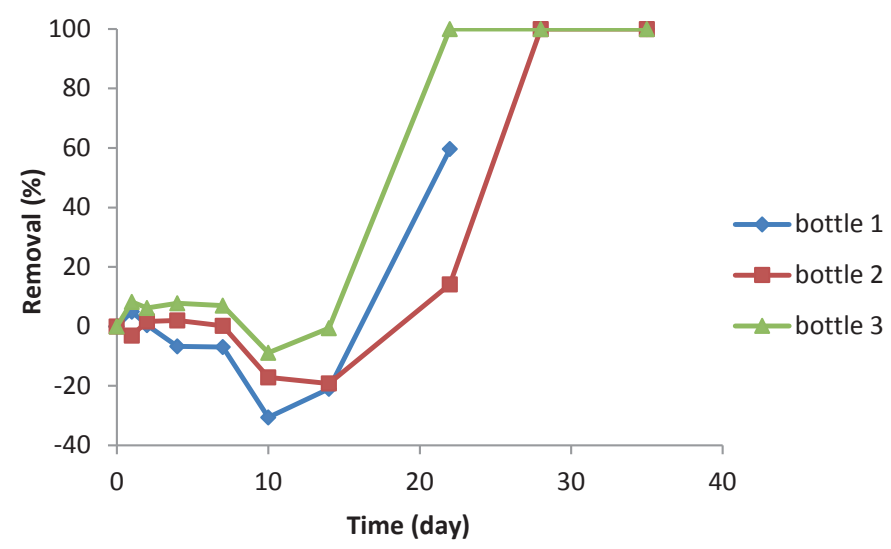

Figure S5.7 Biodegradation of ibuprofen in triplicate bottles of condition C. Sample of bottle 2 at day 28 was missing due to operational mistake.
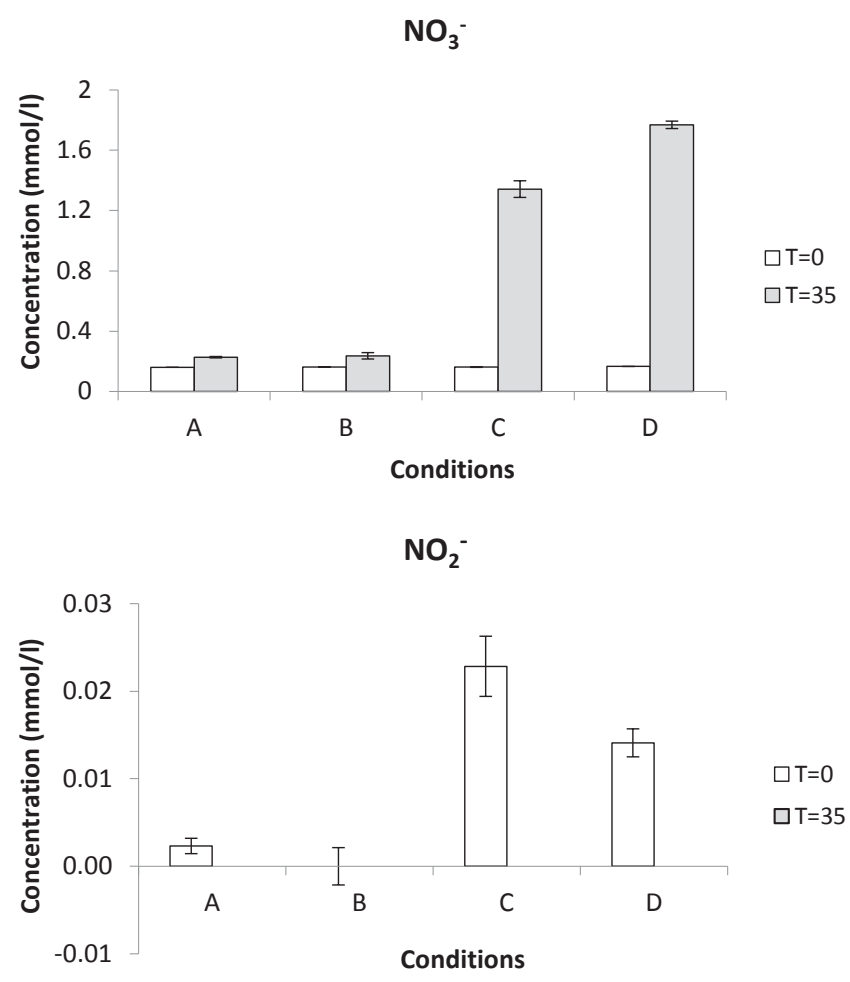


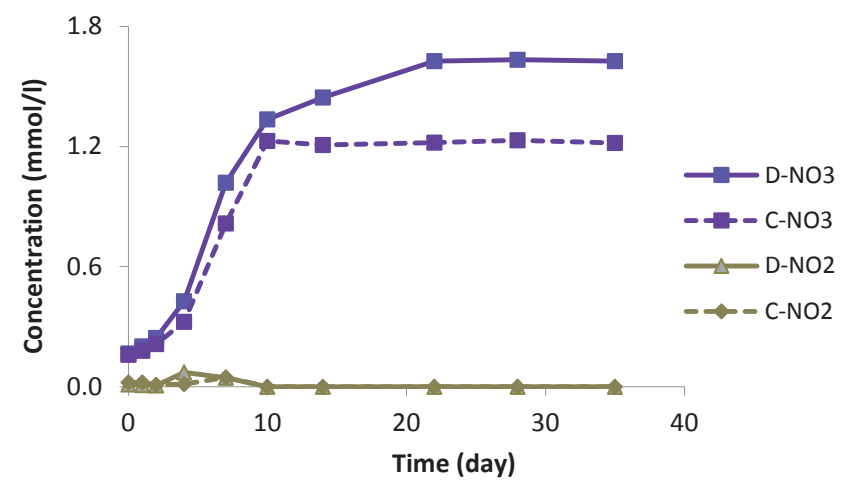

Figure S5.8 Concentrations of $\mathrm{NO}_{3}{ }^{-}$and $\mathrm{NO}_{2}{ }^{-}$in four conditions at day 0 and 35 and their concentrations in conditions $\mathrm{C}$ and $\mathrm{D}$ under nitrification conditions during the 35 days experiments. Results at day 0 and 35 are mean values \pm standard deviation $(n=3)$.

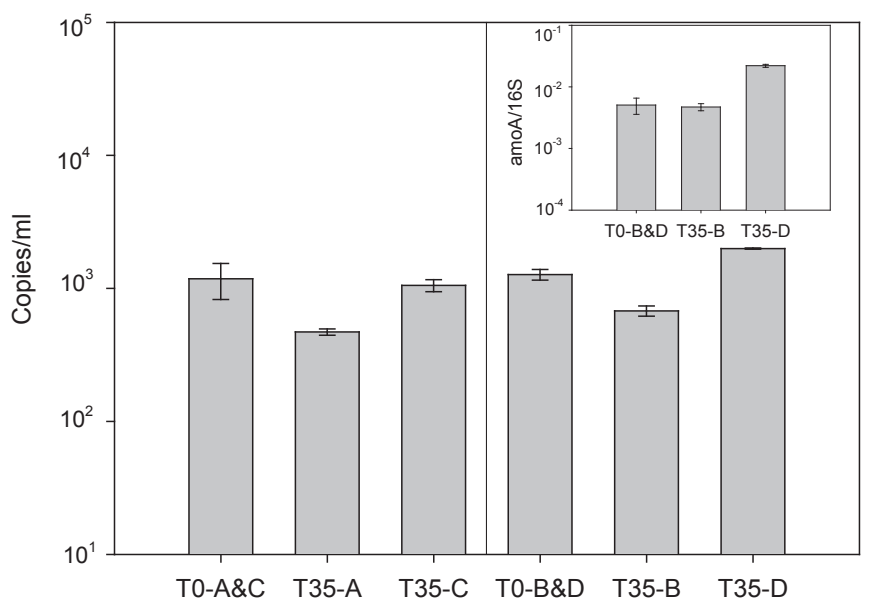

Figure S5.9 Abundance of ammonia monooxygenase (amoA) at day 0 and 35 . The abundance of amoA is represented in absolute concentration and 16S-rRNA normalized values. Microbial source of conditions $\mathrm{A}$ and $\mathrm{C}$ are the enriched microbial culture; microorganism source of conditions $\mathrm{B}$ and $\mathrm{D}$ are the enriched microbial culture and microorganisms from the DOM. Results are mean values \pm standard deviation $(\mathrm{n}=3)$. 
Aerobic biodegradation

Table S5.1 Overview of primer sequencing and thermal cycling conditions for qRCR [12].

\begin{tabular}{|c|c|c|c|c|}
\hline Target & Primers & Sequence $\left(5^{\prime}-3^{\prime}\right)$ & Thermal profile & Cycles \\
\hline $\begin{array}{l}\text { Total } \\
\text { bacteria }\end{array}$ & $\begin{array}{l}341 \mathrm{~F} \\
534 \mathrm{R}\end{array}$ & $\begin{array}{l}\text { CCTACGGGAGGCAGCAG } \\
\text { ATTACCGCGGCTGCTGGC }\end{array}$ & $\begin{array}{l}95^{\circ} \mathrm{C} 10 \mathrm{~min} \\
95^{\circ} \mathrm{C} 15 \mathrm{~s}, 60^{\circ} \mathrm{C} 30 \mathrm{~s}, \\
72^{\circ} \mathrm{C} 40 \mathrm{~s}\end{array}$ & $\begin{array}{l}1 \\
40 \\
\end{array}$ \\
\hline $\begin{array}{l}\text { amoA } \\
\text { bacteria }\end{array}$ & $\begin{array}{l}\text { Bact } \\
\text { amoA-1F } \\
\text { Bact } \\
\text { amoA-2R }\end{array}$ & $\begin{array}{l}\text { GGGGTTTCTACTGGTGGT } \\
\text { CCCCTCKGSAAAGCCTTCTTC }\end{array}$ & $\begin{array}{l}50^{\circ} \mathrm{C} 2 \mathrm{~min}, 95^{\circ} \mathrm{C} 10 \mathrm{~min} \\
95^{\circ} \mathrm{C} 30 \mathrm{~s}, 58^{\circ} \mathrm{C} 40 \mathrm{~s}, \\
72^{\circ} \mathrm{C} 1 \mathrm{~min}\end{array}$ & $\begin{array}{l}1 \\
45\end{array}$ \\
\hline
\end{tabular}

Table S5.2 Biodegradation of metoprolol (\%) in the repeated batch experiment.

\begin{tabular}{lllllll}
\hline \multirow{2}{*}{ Time } & \multicolumn{2}{l}{ Batch with nitrification } & \multicolumn{3}{l}{ Batch without nitrification } \\
\cline { 2 - 7 } & Bottle 1 & Bottle 2 & Bottle 3 & Bottle 1 & Bottle 2 & Bottle 3 \\
\hline Day 9 & -14 & -15 & -14 & -8 & -7 & 31 \\
Day 15 & -6 & -2 & 11 & 12 & 19 & 100 \\
Day 22 & -10 & -5 & 16 & 20 & 29 & 100 \\
\hline
\end{tabular}

Notes: The repeated batch experiment was based on a less inactivated enrichment culture compared to the culture used in the 35 days batch experiment. Still, the results show that metoprolol biodegradation was inhibited under the nitrification conditions. 


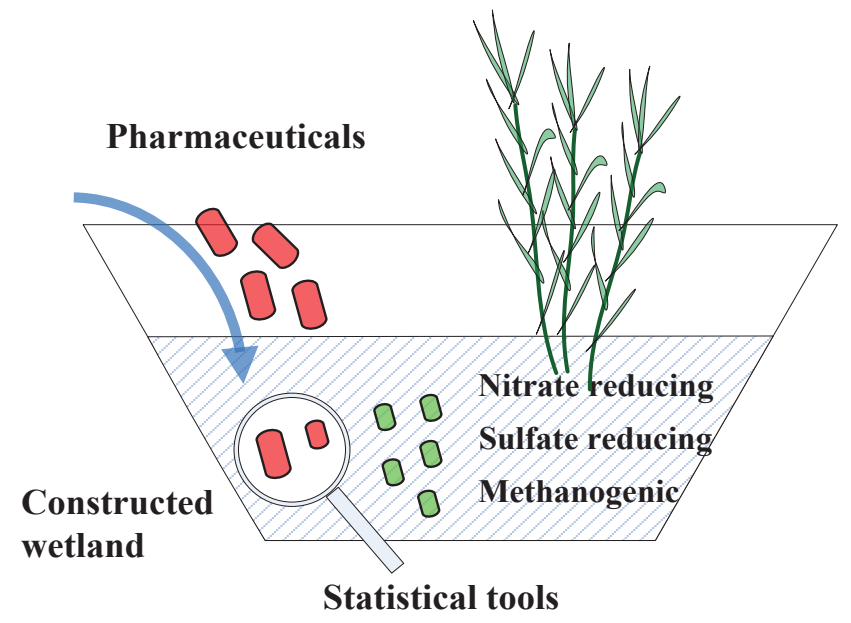




\title{
Chapter 6
}

\section{Pharmaceutical biodegradation under three anaerobic redox conditions evaluated by chemical and toxicological analyses}

\author{
A modified version of this chapter is published as \\ He, Y., Sutton, N.B., Rijnaarts, H.H.M., Langenhoff, A.A.M. Pharmaceutical biodegradation \\ under three anaerobic redox conditions evaluated by chemical and toxicological analyses. \\ Science of the Total Environment, DOI 10.1016/j.scitotenv.2017.07.219.
}




\begin{abstract}
So far, biodegradation of pharmaceutically active compounds (PhACs) in the subsurface layer of constructed wetlands (CWs) under various anaerobic redox conditions is rarely studied. In this study, CW sediment microbial populations were enriched for PhAC biodegrading organisms. Biodegradation effectivity of a mixture of six PhACs (caffeine, CAF; naproxen, NAP; metoprolol, MET; propranolol, PRO; ibuprofen, IBP; carbamazepine, $\mathrm{CBZ}$ ) and single compounds (CAF, NAP) was investigated under nitrate reducing, sulfate reducing, and methanogenic conditions using chemical and toxicological analyses. Biodegradation performance varied strongly among the six PhACs and three redox conditions chosen. CAF and NAP were completely biodegraded under sulfate reducing and methanogenic conditions whereas biodegradation efficiencies of the other PhACs are much less (MET, PRO <20\%; IBP, CBZ, negligible). CAF and NAP showed significantly lower biodegradation under nitrate reducing conditions than under the other two redox conditions. No difference was found in biodegradation efficiencies of CAF and NAP when present as single compound, or as a mixture with other PhACs. Different intermediates were observed, indicating different biodegradation pathways under different redox conditions and when the PhACs were present as single or in a mixture. From toxicological perspective, toxicity of $\mathrm{PhACs}$ and/or their intermediates to Vibrio fischeri was attenuated during the biodegradation process. Chemical and toxicological data showed positive correlations in principle component analysis, by which potentially toxic PhACs and intermediates are screened as candidates for further ecotoxicological hazard assessment.
\end{abstract}

\title{
Keywords
}

Pharmaceuticals; Constructed wetlands; Anaerobic biodegradation; Intermediates; Toxicity; Correlation 


\subsection{Introduction}

The occurrence and adverse effects of pharmaceutically active compounds (PhACs) in the environment becomes one of the emerging environmental problems [118]. Wastewater treatment plants (WWTPs) are the main barrier to prevent PhACs from being discharged into surface water [190]. Unfortunately, conventional WWTPs are not (yet) designed to efficiently remove PhACs [359]. Thus, residual PhACs and intermediates formed in WWTPs are discharged to aquatic ecosystems. As reported in a recent study, $37 \mathrm{PhACs}$ were detected at $113 \mathrm{ng} / \mathrm{L}$ (median) in source water samples from 25 American drinking water treatment plants [133]. Although PhACs in the environment are present at low concentrations (ng/l or $\mu \mathrm{g} / \mathrm{l})$ and are unlikely to reach lethal toxicity [94], their persistent accumulation may lead to observable toxicological effects in key organisms in aquatic or terrestrial ecosystems after long-term exposure [227]. For example, the lowest observed effect concentration of propranolol (PRO) affecting reproduction in Ceriodaphnia dubia (water flea) was $250 \mu \mathrm{g} / \mathrm{L}$, and reproduction in Hyalella azteca (crustacean) was affected at $100 \mu \mathrm{g} / \mathrm{L}$ after 27 days longterm exposure [178]. Although these effects are demonstrated at high concentrations, the effect of cocktails of a multitude of compounds is still unknown. Hence, cost efficient removal of PhACs from WWTP effluents is important and constructed wetlands (CWs) are attractive for this purpose.

CWs are engineered systems designed to use natural processes to treat contaminants [370]. It is reported that $\mathrm{CW}$ could be a sustainable, cost-efficient and easily maintained and operated system as a post-treatment process for PhAC removal [200]. A complicated interplay of physical, chemical and biological processes take place in CWs, such as photodegradation, phytoremediation, sorption and biodegradation. Among those processes, biodegradation plays a main role in the transformation and mineralization of micropollutants including PhACs [224]. Biodegradation of PhACs has been demonstrated and revealed a strong correlation with the availability of electron acceptors and redox conditions [334]. In the subsurface layer of CWs, due to the limited oxygen concentration, anaerobic processes predominate and different redox conditions take place simultaneously, such as nitrate reducing, sulfate reducing, and methanogenic conditions [334].

Microbial community composition is dictated by the availability of electron acceptors that vary with specific redox conditions, and select for specific biodegradation processes [119]. Most of the studies addressing PhAC biodegradation focused on the aerobic biodegradation 
and the indigenous microbial populations present in the sediment and soil [221, 231, 392, 406]. In comparison, investigations of $\mathrm{PhAC}$ biodegradation under specific anaerobic redox conditions are scarce, and rarely focus on redox specific microbial populations in $\mathrm{CW}$ sediment. When looking at the effect of redox conditions on PhAC biodegradation, the limited studies mainly discuss the effect by comparing the biodegradation rates of parent compounds [81]. However, different microbial communities can be selected by different redox conditions, which may result in differences not only in biodegradation rates but also in biodegradation pathways. As a previous study observed, different intermediates of ${ }^{14} \mathrm{C}$ radiolabelled sulfamethoxazole were produced under aerobic, anoxic, and anaerobic conditions [5]. Toxicity of those varied intermediates is much less unknown compared with the parent $\mathrm{PhACs}$ [60]. Therefore, it is essential to gain more knowledge of $\mathrm{PhAC}$ anaerobic biodegradation under specific redox conditions by chemical and toxicological analyses of parent PhACs and their intermediates.

In this study, batch experiments were performed to explore the effect of specific anaerobic redox conditions on $\mathrm{PhAC}$ biodegradation by chemical and toxicological analyses. Six widely consumed PhACs with different physicochemical properties were selected as the target compounds. As PhACs often present in a variety of mixture, biodegradation of PhACs presence as single or as a mixture with other PhACs were compared to investigate the interaction between PhACs. This research thus aims to provide insight into PhAC biodegradation processes in the subsurface layer of CWs, and to assess their contribution to reduce hazardous effects of PhACs and intermediates to aquatic ecosystems.

\subsection{Materials and Methods}

\subsubsection{Chemicals and reagents}

PhACs were purchased from Sigma-Aldrich (USA): metoprolol (MET), caffeine (CAF), PRO, carbamazepine (CBZ), naproxen (NAP), ibuprofen (IBP), and fenoprofen calcium salt (internal standard). Mixed PhAC stock $(20 \mathrm{mg} / \mathrm{L})$ was prepared with MilliQ water (Millipore, USA). Acetonitrile with $0.1 \%$ formic acid, water with $0.1 \%$ formic acid, and methanol (Biosolve B.V., the Netherlands) were used for ultra-performance liquid chromatography (UPLC) analysis. All the other chemicals used are of analytical grade. 


\subsubsection{Batch experiment}

Batch experiments were conducted to study $\mathrm{PhAC}$ biodegradation at different redox conditions with individual PhACs and a mixture of PhACs. Three types of media were prepared according to previous works for nitrate reducing [112], sulfate reducing [212], and methanogenic conditions [174]. As inoculum, sediment was collected from two CWs that are in use for post-treatment processes of two Dutch WWTPs and cultivated with a PhAC mixture ( $1 \mathrm{mg} / 1$ each) under three redox conditions for 15 months. The culture was amended with $\mathrm{PhAC}$ mixture repeatedly during cultivation. After these 15 months, $10 \%$ of the enrichment cultures from three redox conditions were transferred twice in 6 weeks separately. Microorganisms were active before transfer by removing more than $95 \%$ of CAF and NAP. By transferring, a liquid enrichment culture without sediment was obtained and more active microorganisms for PhAC biodegradation were expected to be selected.

For each redox condition, three batch groups were designed with either a mix of six PhACs, or single CAF or NAP, which makes nine groups in total. CAF and NAP were selected to investigate the biodegradation when present as single or mixed resulting from their higher biodegradation compared with other PhACs during the enrichment. In each group, biotic batches were conducted in triplicate and abiotic controls in duplicate. Abiotic batches were autoclaved at $120{ }^{\circ} \mathrm{C}$ for 20 minutes with four times continuous repetition. Additionally, chemical inhibitors were added to further inhibit microbial activity in abiotic controls $(0.3$ $\mathrm{g} / \mathrm{L} \mathrm{HgCl}_{2}$ and $1.3 \mathrm{~g} / \mathrm{L} \mathrm{NaN}_{3}$ ). In each batch bottle, $10 \mathrm{ml}$ liquid inoculum was mixed with $110 \mathrm{ml}$ medium spiked with a mixture of six PhACs ( $1 \mathrm{mg} / 1 \mathrm{each})$, or only CAF or NAP (1 $\mathrm{mg} / \mathrm{l})$. PhAC stock was prepared in water to ensure PhACs were the only carbon source added. Gas phase in the batch bottles was $\mathrm{CO}_{2} / \mathrm{N}_{2}(20 / 80$, v/v). Batch bottles were incubated on a shaker $(120 \mathrm{rmp})$ at $20{ }^{\circ} \mathrm{C}$. To avoid photodegradation of PhACs, all bottles were covered with aluminium foil. The batch experiments lasted for 10 weeks and samples were taken at week 0, 3, 6, 8, 10 for chemical and toxicological analyses. Since there were PhACs incompletely biodegraded in the enrichment period, the residual PhACs were transferred to the following batches inevitably.

\subsubsection{Chemical analysis}

UPLC (Ultimate 3000, Thermo, USA) equipped with diode array detection (DAD) was used to measure $\mathrm{PhAC}$ concentrations. Liquid samples were pre-treated by centrifugation at 10000 rpm for $5 \mathrm{~min}$ and spiked with fenoprofen as internal standard prior to UPLC-DAD analysis. 


\section{Anaerobic biodegradation}

The analysis method followed the description of a previous study [156]. Quantification of PhACs was conducted based on the internal standard and external calibration standards.

Concentrations of $\mathrm{NO}_{3}{ }^{-}$and $\mathrm{SO}_{4}{ }^{2-}$ under nitrate and sulfate reducing conditions were analyzed by an IC (Dionex ICS-2100, Thermo, USA) as described in De Wilt et al. [375]. Concentrations of $\mathrm{CO}_{2}$ were followed by a gas chromatography (GC-2010, Shimadzu, Japan) according to the method described in in De Wilt et al. [375].

\subsubsection{Toxicological analysis}

Microtiter Microtox bioassays were conducted to evaluate the toxicity variation of PhACs during the biodegradation process. The bioassay was performed as described by $\mathrm{He}$ et al. [156]. Luminescent bacteria Vibrio fischeri was purchased from Microlan (the Netherlands). Luminescence of $V$. fischeri was recorded by a plate reader (Tecan infinite M200 PRO, Switzerland). Liquid samples were pre-treated with centrifugation at $10000 \mathrm{rpm}$ for $5 \mathrm{~min}$ and analysed in triplicate. $\mathrm{CuSO}_{4}$ was set as the positive control and redox media as the blank control.

\subsubsection{Statistical analysis}

The correlation between $\mathrm{PhAC}$ biodegradation and toxicity variation during the biodegradation was conducted by principle component analysis (PCA) using CANOCO 5 software package (Biometrics, the Netherlands). Response variables in PCA were centralized and standardized to achieve zero average and unit variance. Statistical differences of PhAC toxicity between days were established by the analysis of variance method (ANOVA, single factor) at different significance levels. Comparisons were considered significantly different for $* \mathrm{p}<0.05, * * \mathrm{p}<0.01$ and $* * * \mathrm{P}<0.001$.

\subsection{Results and discussion}

\subsubsection{Biodegradation of PhACs under different redox conditions}

To confirm biological activity was associated with the applied redox conditions, concentrations of electron acceptors were measured, including $\mathrm{NO}_{3}{ }^{-}, \mathrm{SO}_{4}{ }^{2-}$ and $\mathrm{CO}_{2}$. Decrease of those electron acceptors was observed in biotic batches, which illustrates that the related nitrite reducing, sulfate reducing and methanogenic conditions did occur (Table S6.1). 


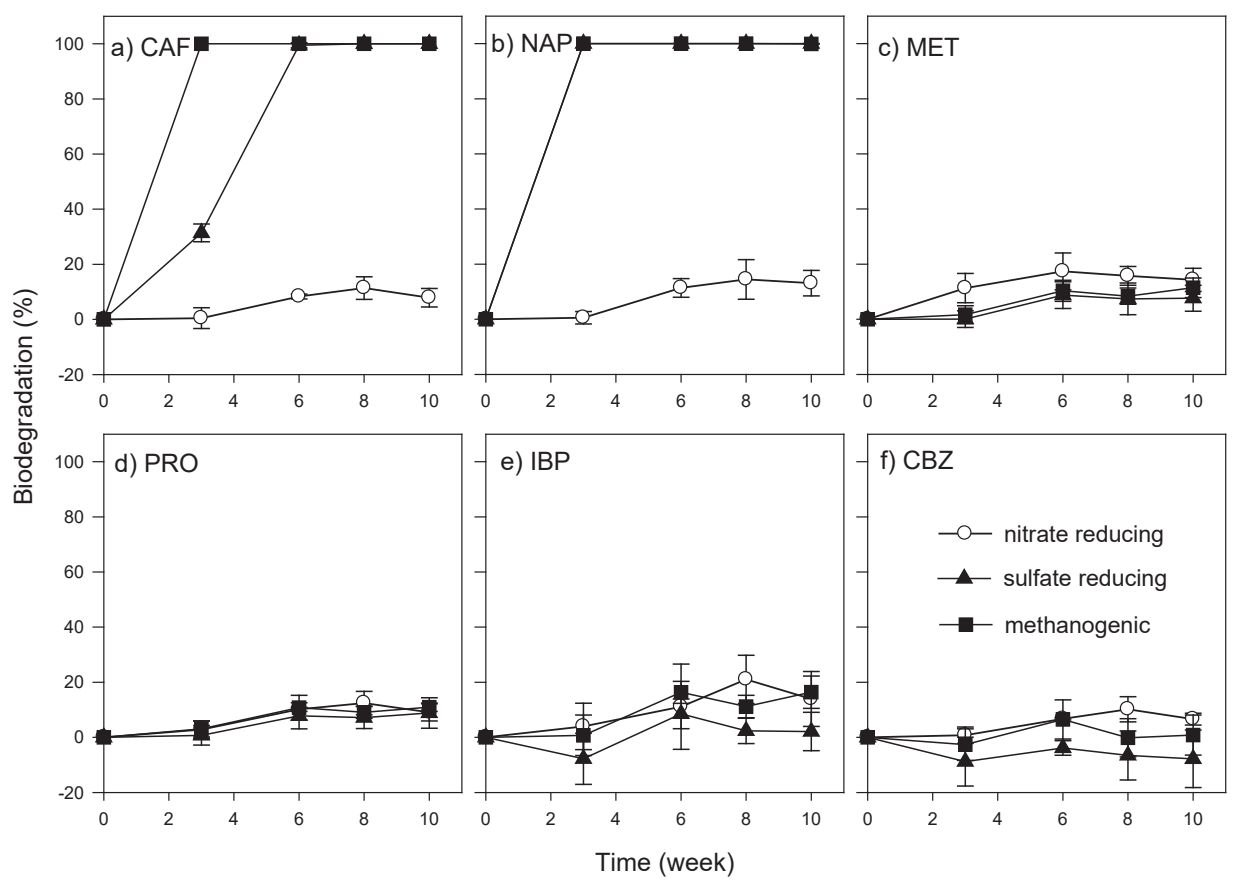

Figure 6.1 Biodegradation of PhACs in three redox conditions under mixed status a) CAF; b) NAP; c) MET; d) PRO; e) IBP; f) CBZ. Only two lines are visible for NAP biodegradation as the tendency under sulfate reducing and methanogenic conditions are overlapped. Data are mean biodegradation \pm standard error $(n=3)$.

Biodegradation of PhACs showed discrepancies for different PhACs. CAF and NAP were completely degraded under sulfate reducing and methanogenic conditions (Figure 6.1). Our observation of readily CAF removal is in line with literature. For example, high removal of CAF ( $>90 \%$ ) was reported for various treatment techniques with different electron acceptors including the activated sludge process, upflow anaerobic sludge blanket reactors, and stabilization lagoons [132]. NAP was found to remain unchanged in soil batches under anaerobic conditions during 84 days cultivation without pre-enrichment [231]. In comparison, the higher NAP biodegradation in our study may have been a result from the natural inoculum present in the sediment we selected and the 15 months microbial enrichment. The wetland sediment we used had regular exposure to PhACs from WWTP effluents, and could have therefore an enriched PhAC degrading microbial population. Biodegradation of MET and PRO were lower than 20\% for all three redox conditions (Figure 6.1). Similar with our study, MET and PRO showed negligible biodegradation in soil batches under nitrate reducing 
conditions in a study of Barbieri et al. [22]. IBP and CBZ were recalcitrant to biodegradation under all the investigated redox conditions (Figure 6.1). The major removal mechanism of IBP has been reported to be aerobic biodegradation [15], which is consistent with our observation of low IBP biodegradation in our anaerobic study. CBZ is known to be a persistent and difficult to be biodegraded under either aerobic or anaerobic conditions. The decrease of $\mathrm{CBZ}$ is mainly due to the sorption process [333]. Compared with the biotic experiments, PhAC biodegradation in abiotic controls was negligible (Figure S6.1).

Biodegradation of PhACs varied among redox conditions tested. Nitrate reducing conditions showed significantly lower biodegradation of CAF and NAP compared with the other two redox conditions. CAF biodegradation was faster under methanogenic than under sulfate reducing conditions (Figure 6.1). To date, there is few research reports comparison of PhAC biodegradation under various anaerobic redox conditions. The limited studies of estrogen anaerobic biodegradation showed contradicting outcomes. Mashtare et al. [255] found that biodegradation of $17 \alpha$-estradiol and $17 \beta$-estradiol under nitrate reducing conditions was several times higher than under sulfate reducing conditions. However, another estrogen research reported that biodegradation rate of $17 \beta$-estradiol was fastest under sulfate reducing conditions, followed by methanogenic conditions, and slowest under nitrate reducing conditions [85]. It is of interest to compare to other contaminants such as toluene that have been studied for decades and contain aromatic rings like many PhACs. Biodegradation rates of toluene increase with the prevailing oxidation potential (i.e., nitrate reducing $>$ sulfate reducing $>$ methanogenic conditions) $[83,211,304]$. Our study shows a different redox effect (methanogenic $>$ sulfate reducing $>$ nitrate reducing conditions), which may result from the PhACs we investigated are with different physicochemical properties.

In addition, we observed a variation of the transferability of degradation capacity. Capacity of CAF biodegradation was lost for all three redox conditions during transfer (Table S6.2). However, the capacity recovered under sulfate reducing and methanogenic conditions in the batch period but not under nitrate reducing conditions. Additionally, capacity loss of NAP biodegradation only occurred under nitrate reducing conditions did not recovered afterwards. Therefore, it seems that the microorganisms capable of degrading PhACs are more robust under sulfate reducing and methanogenic conditions compared with nitrate reducing conditions, and we did not found a report on that in previous studies. 
Production of different intermediates was observed under different redox conditions. Sulfate reducing and methanogenic conditions showed similar removal efficiencies for all the investigated PhACs (Figure 6.1) while a significant difference was observed in the intermediate production (Figure S6.2). Only two common intermediates (RT=8.61, $10.87 \mathrm{~min}$ ) were found among the 17 intermediates detected under sulfate reducing conditions and 10 intermediates under methanogenic conditions (Figure S6.2), assuming that one retention time (RT) represents one unique substance in the chromatograph. The observed variation in intermediates may indicate different biodegradation pathways, which may result from the selection pressure of redox conditions. Therefore, when evaluating the effect of redox conditions, exploring the variation of intermediates is essential apart from comparing biodegradation rates of the parent compounds.

\subsubsection{Biodegradation of PhACs present as single compound or in a mixture of PhACs}

Biodegradation efficiencies of CAF and NAP showed no significant differences when present as single or in a mixture with other PhACs under any of the investigated redox conditions (Figure S6.3). This is in contrast with previous studies showing that degradation rates of individual compounds are higher than for those present in a mixture $[6,277]$.

Differences were found in the intermediates of CAF and NAP for the two situations, indicating different biodegradation pathways. CAF was completely biodegraded under methanogenic conditions both alone and in a mixture. 18 intermediates were detected when CAF was the single compound, while only three out the 18 intermediates (labelled in Figure 6.2) were observed in the mixed compound incubations (Figure S6.2). Similar unequal intermediate patterns were observed when comparing the $\mathrm{CAF}$ intermediates under nitrate and sulfate reducing conditions for both single (Figure S6.4) and mixed compound studies (Figure S6.2, Figure 6.3b). The same was found for NAP intermediates for both single (Figure S6.5) and mixed PhACs studies under all three redox conditions (Figure S6.2, Figure $6.3 \mathrm{~b})$. Additionally, the message delivered from unequal intermediate production in the single and mixed conditions are complicated. To understand the exact mechanism, more chemical analyses are needed for intermediate identification. 


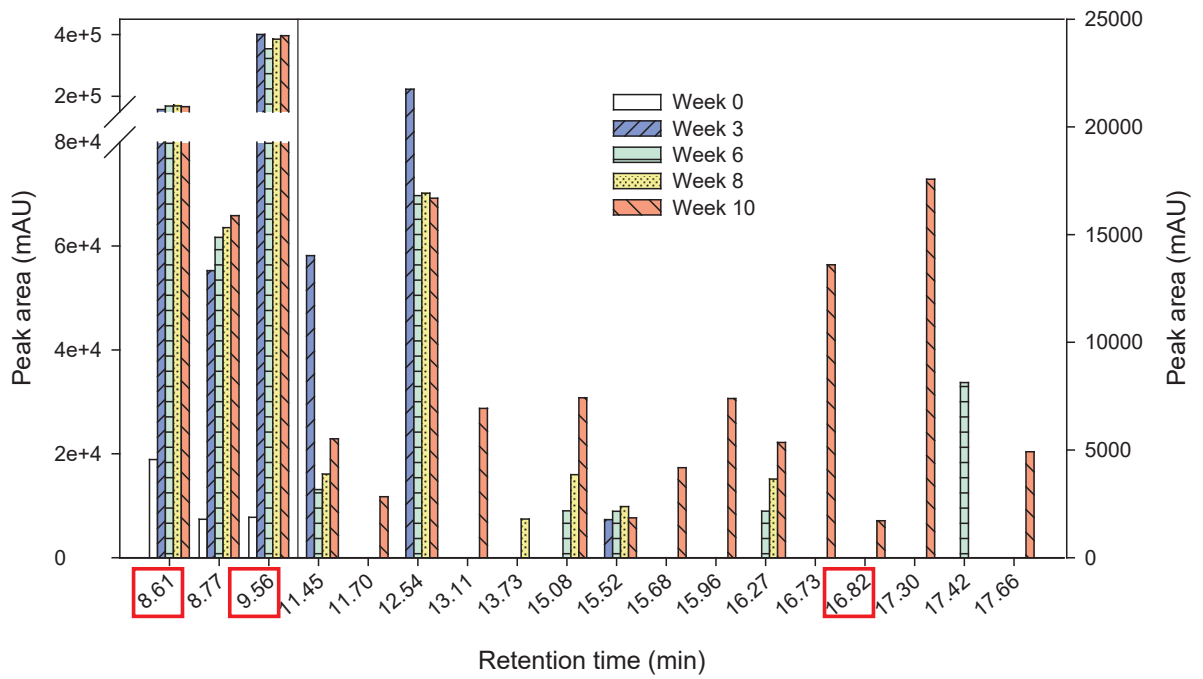

Figure 6.2 Production of intermediates in single CAF experiment under methanogenic conditions showed in the chromatography spectrum. Chromatography peaks detected in the redox medium were excluded. Intermediates with RT from 11.45 to $17.66 \mathrm{~min}$ are represented in the right axis due to the relatively low peak area. Intermediates labelled with red rectangle were detected in mixed CAF experiment under methanogenic conditions as well.

\subsubsection{Toxicological analysis of PhAC biodegradation}

Toxicity of PhACs and their intermediates was evaluated during the biodegradation. In general, toxicity of mixed PhACs to $V$. fischeri was attenuated during all three anaerobic biodegradation processes (Figure 6.3a). After being treated by anaerobic biodegradation, the mixture of $\mathrm{PhACs}$ and/or their intermediates were less toxic, or even non-toxic under sulfate reducing and methanogenic conditions. Toxicity of single CAF and NAP showed similar decrease tendency with the mixed studies (Figure S6.6). Interestingly, toxicity of mixed PhACs under nitrate reducing conditions decreased significantly (approximate 40\%) after 10 weeks (Figure 6.3a), even though the related biodegradation efficiencies of parent PhACs were lower than 20\% (Figure 6.1). The initial toxicity of PhACs was higher under nitrate reducing conditions compared with the other two conditions (Figure 6.3a), which might be caused by the residual PhACs and/or their intermediates transferred from the enrichment culture. The higher initial and final toxicity of PhACs at week 10 under nitrate reducing conditions indicates more incomplete biodegradation of parent compounds or formation of more toxic intermediates either during the enrichment or in the 10 weeks period. 

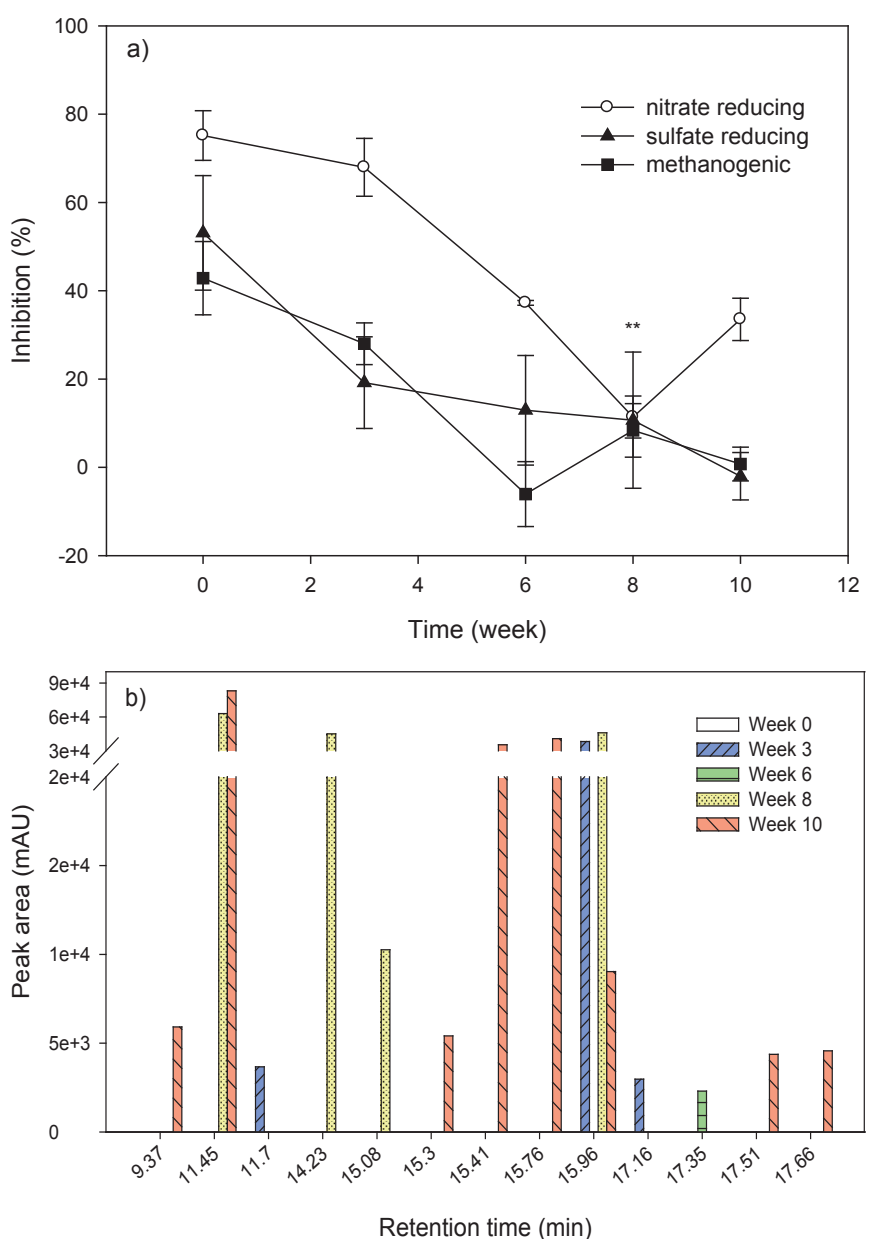

Figure 6.3 Toxicity variation of PhACs and/or their intermediates to V. fischeri. a) toxicity in three redox conditions under mixed status. Data are mean inhibition \pm standard error $(n=3)$; b) intermediates of mixed PhACs detected under nitrate reducing conditions. Chromatography peaks detected in the redox medium were excluded.

In addition to reflecting the biodegradation of parent $\mathrm{PhACs}$, toxicity results also give insight into the production of toxic intermediates. Biodegradation of parent $\mathrm{PhACs}$ does not mean that the toxicity will decrease accordingly, as more toxic intermediates can be formed. From week 8 to week 10, toxicity under nitrate reducing conditions increased significantly $(\mathrm{p}=0.002)$ indicating production and/or accumulation of toxic intermediates. According to the chromatography results (Figure 6.3b), intermediates with $\mathrm{RT}=9.37,15.30,15.41,15.76$, 
17.51 and $17.66 \mathrm{~min}$ appeared at week 10 and the intermediates with $\mathrm{RT}=11.45 \mathrm{~min}$ accumulated in week 10 . Those intermediates may have contributed to the observed toxicity increase.

\subsubsection{Correlation between chemical and toxicological data}

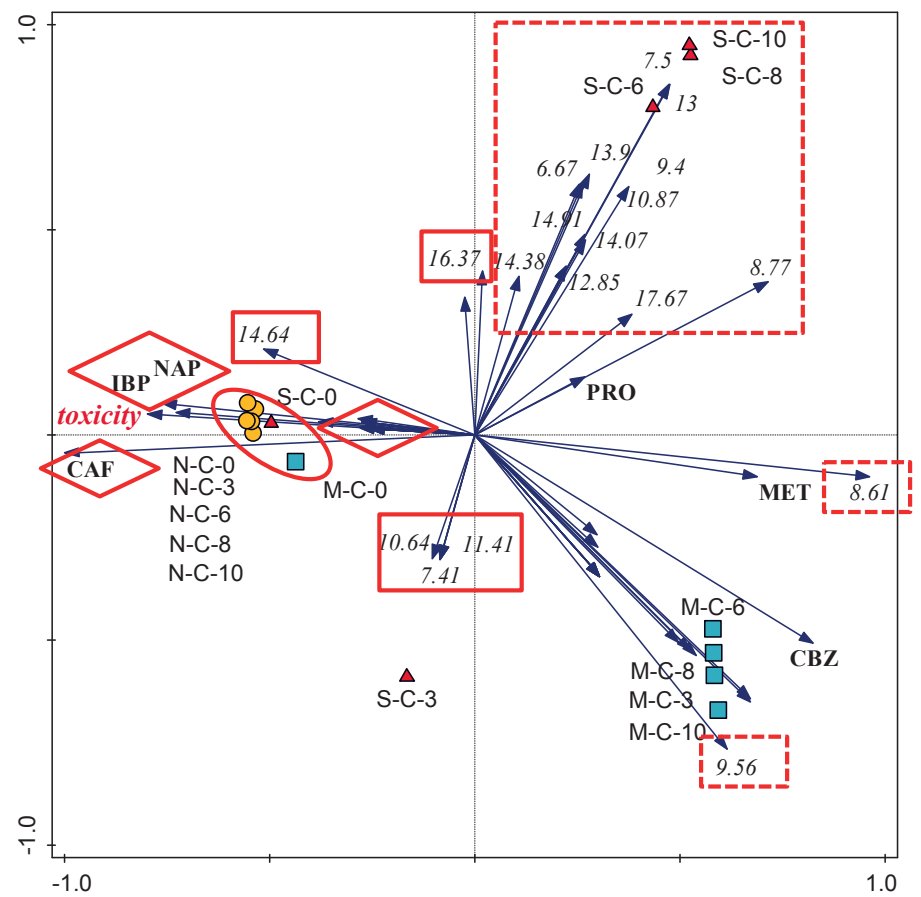

Figure 6.4 Principle component analysis of the correlation between results of chemical analysis and toxicological data of single CAF experiment under three redox conditions during 10 weeks. N-C (circles), S-C (triangles), and M-C (rectangles) represent for single CAF status under nitrate reducing, sulfate reducing, and methanogenic conditions, respectively. Environmental variables including toxicity, PhACs and their intermediates are shown in arrows. The length of arrows indicates variance that can be explained by the environmental variables while the direction of arrows indicates the magnitude of variables. The perpendicular distance between samples and variable arrows represent their correlations, in which smaller distance means stronger correlations. The intersection angles between arrows represent their correlations, in which more acute intersection angle means stronger correlations. The eigenvalues of the first and second canonical axis are 0.93 and 0.05 . 
Chemical analysis or toxicological analysis alone is not sufficient for deriving an accurate profile of the possible ecotoxicological hazard of PhACs and/or their intermediates in an environmental sample [120]. To identify (unknown) hazardous compounds in a sample, the effect-directed analysis (EDA) technique has been developed, which is based on a combination of bioassays, fractionation and chemical analysis [43]. However, a drawback of the EDA method is that a number of fractions need to be collected and confirmed individually, which is expensive and time-consuming [43]. Therefore, it is critical to screen for components in the sample which may actually contribute to the toxicity. In this study, statistical tools were used to explore the correlations between chemical and toxicological data, as a basis for screening possible toxic components.

Chemical and toxicological data showed a positive correlation by PCA. Taking the single CAF study as an example, samples from nitrate reducing conditions correlated positively to toxicity during the biodegradation process (ellipse, Figure 6.4). In comparison, only the week 0 sample showed positive correlation with toxicity under sulfate reducing and methanogenic conditions (Figure 6.4). Those conclusions are in line with the toxicity variation shown in Figure S6.6.

Based on the positive correlation, PCA as an analytical tool can identify which components mostly contribute to the detected toxicity under the three investigated redox conditions. As shown in Figure 6.4 (diamond), toxicity mainly correlates with CAF, NAP, IBP and intermediates produced under the nitrate reducing conditions (linked to Figure S6.5). Additionally, PCA could primarily narrow the identification of toxic compounds in a sample for hazard assessment. For example, chromatography results showed 21 intermediates in the single CAF experiment under sulfate reducing conditions (Figure S6.5). By applying PCA, intermediates with $\mathrm{RT}=7.41,10.64,11.41,14.64,16.37$ min could be screened as potential toxicants as they are positively correlated to toxicity (solid rectangle, Figure 6.4), while the other intermediates could be disregarded (dash rectangle, Figure 6.4). Our findings show that PCA of chemical and toxicological data can provide an initial screening of the hazards in a sample, which can be used as a primary screening tool narrowing down the number of compounds to be evaluated by the labour intensive EDA analyses. 


\subsection{Conclusions}

To investigate $\mathrm{PhAC}$ biodegradation and related hazardous assessment in the subsurface layer of CWs, biodegradation of PhACs was studied under various anaerobic redox conditions by chemical and toxicological analyses. The following conclusions can be drawn:

- $\mathrm{CAF}$ and NAP were completely degraded under sulfate reducing and methanogenic conditions. Degradation efficiencies of MET and PRO were lower than 20\% under all studied conditions. IBP and CBZ were recalcitrant to biodegradation under all three redox conditions.

- Nitrate reducing conditions showed significantly lower biodegradation of CAF and NAP compared with the other two redox conditions. Different intermediates were observed under sulfate reducing and methanogenic conditions which may indicate different biodegradation pathways, even though those two conditions showed similar biodegradation efficiencies for the investigated PhACs.

- Biodegradation efficiencies of CAF and NAP showed no significant differences when present as single compound or in mixed PhAC incubations. However, different biodegradation pathways appear to be active as indicated by differences in intermediates detected.

- In general, toxicity of PhACs and/or their intermediates to $V$. fischeri was attenuated during the biodegradation process. In addition to reflecting biodegradation of parent $\mathrm{PhACs}$, toxicity results also give insight to the production of toxic intermediates.

- PCA identified positive correlations between groups of chemical and toxicological data. PCA as an analytical tool could primarily narrow down the number of compounds in a sample for further identification of toxic hazard assessment, e.g. EDA.

\section{Acknowledgement}

Authors thank Xinchen Niu for her practical support during the batch experiments. The support provided by China Scholarship Council (CSC) for the research of Yujie He at Wageningen University is kindly acknowledged. 


\section{Supplementary materials}
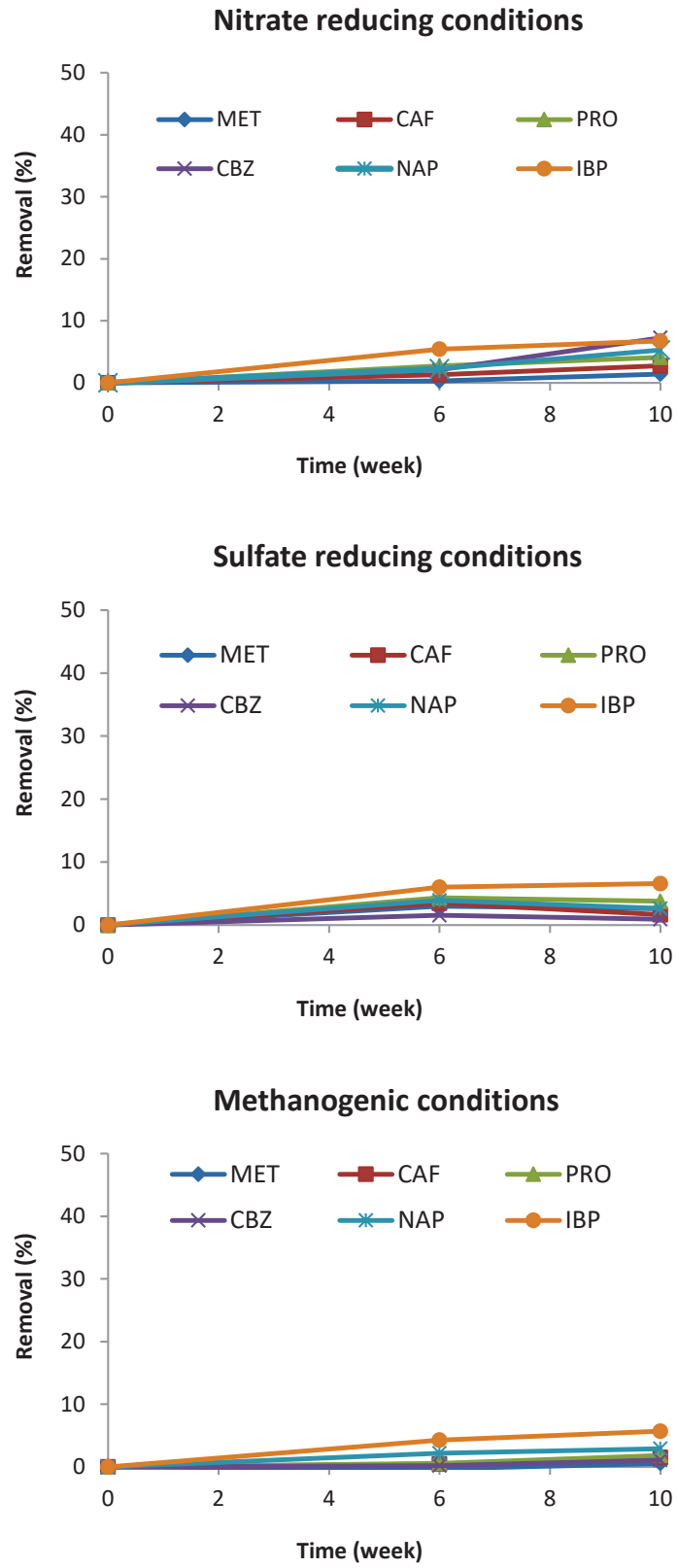

Figure S6.1 Abiotic removal of PhACs under three redox conditions. 

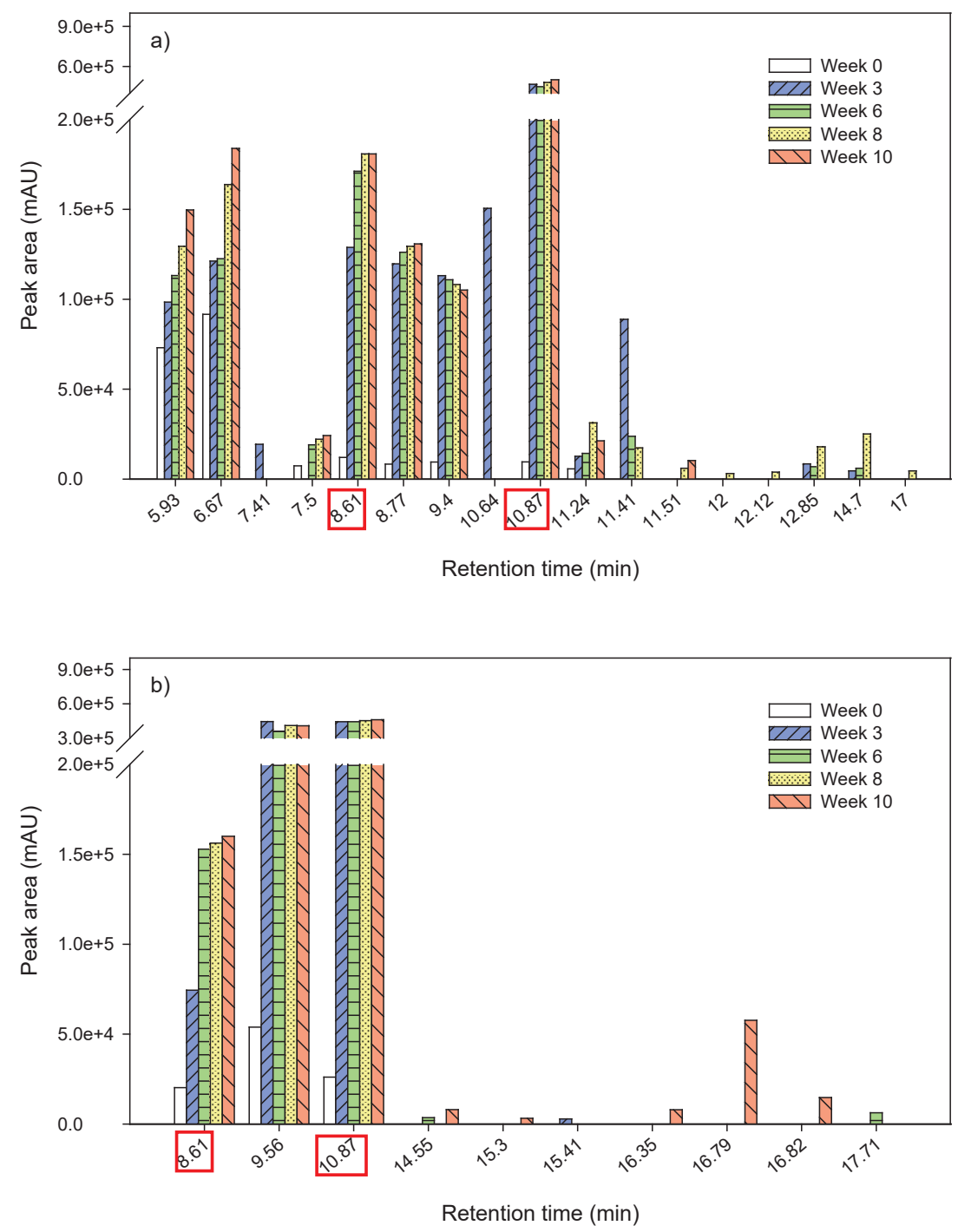

Figure S6.2 Production of intermediates in mixed PhACs under a) sulfate reducing and b) methanogenic conditions showed in the chromatography spectrum. Chromatography peaks detected in the redox medium were excluded. Intermediates labelled with red rectangle were detected in both conditions. 

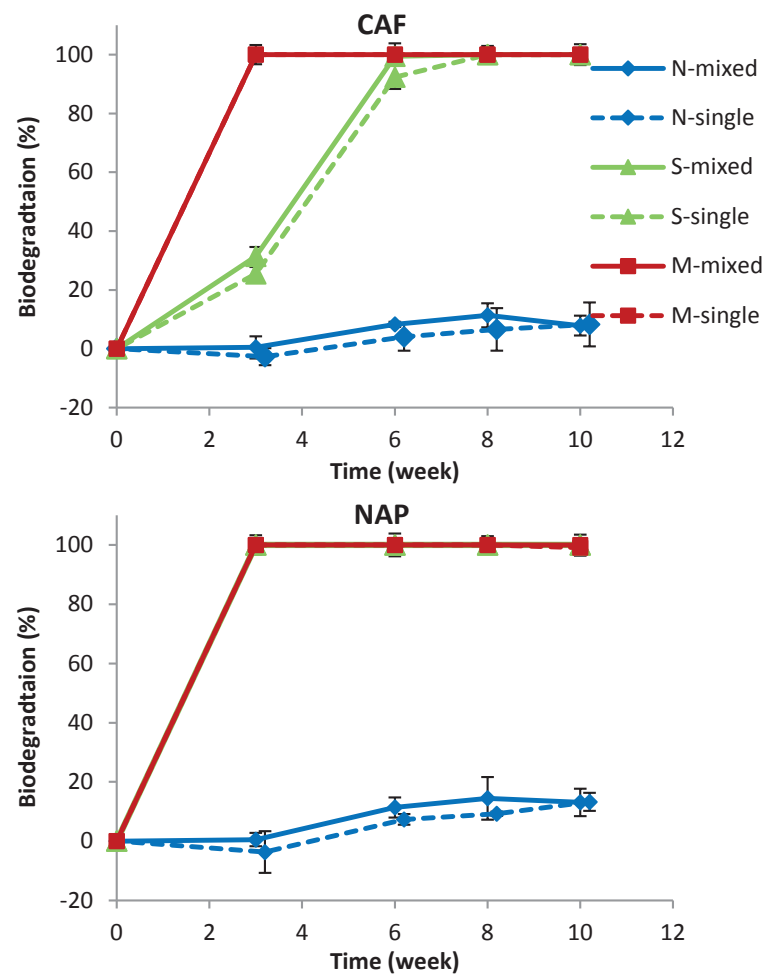

Figure S6.3 Biodegradation of CAF and NAP in three redox conditions under mixed and single status. $\mathrm{N}, \mathrm{S}, \mathrm{M}$ represent nitrate reducing, sulfate reducing and methanogenic conditions. Data are mean removal \pm standard error $(\mathrm{n}=3)$.

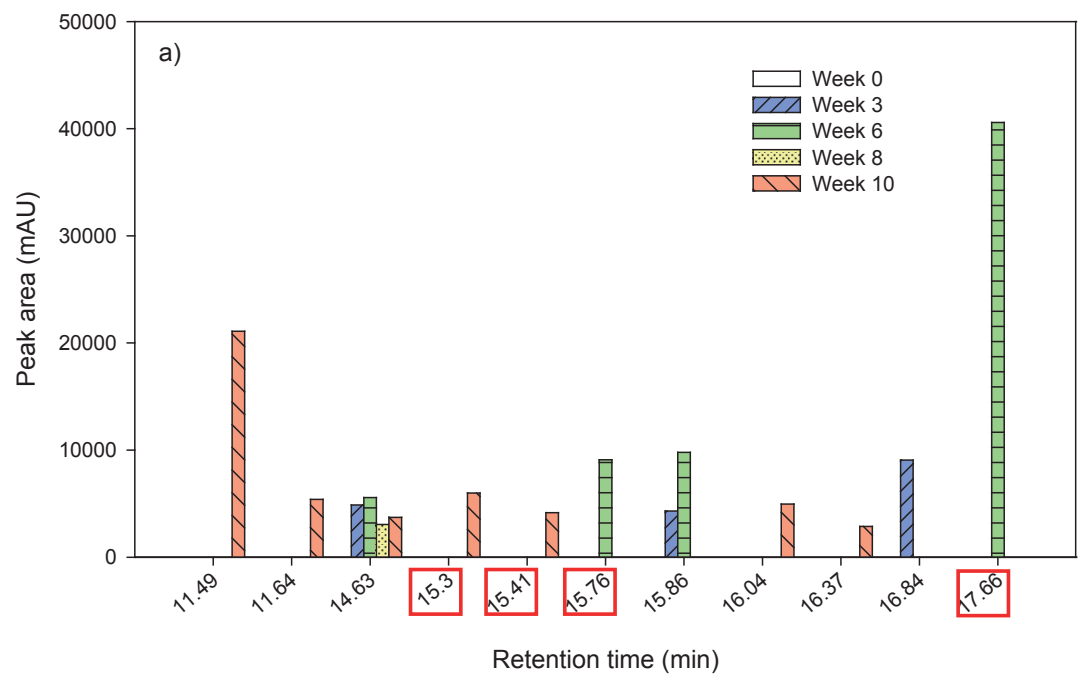




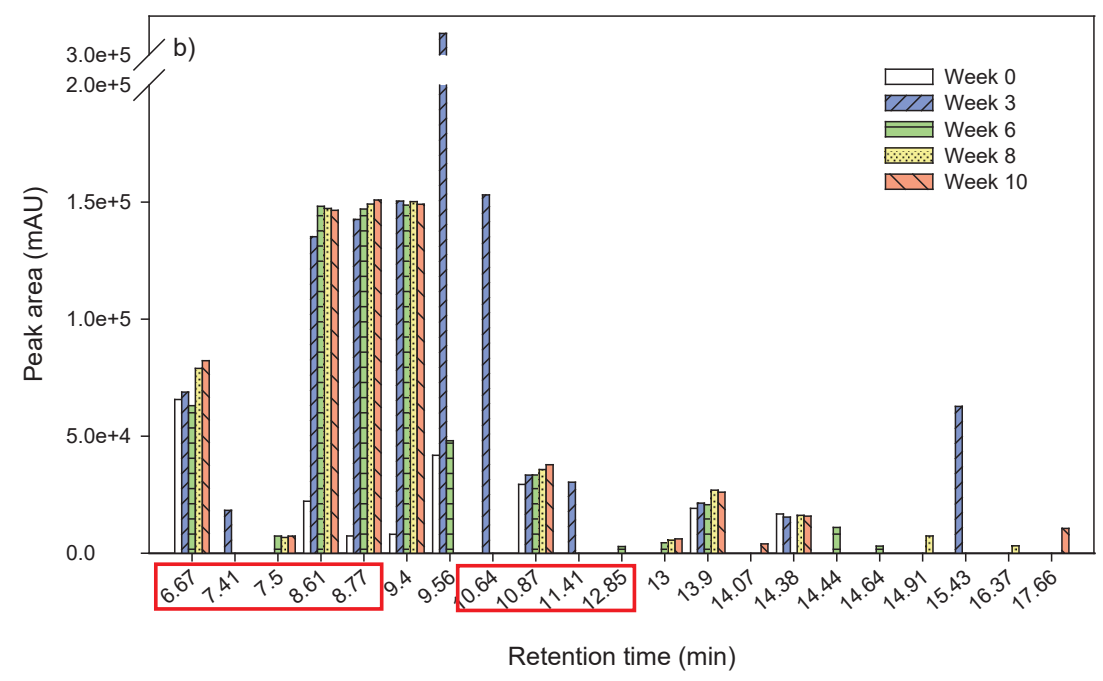

Figure S6.4 Production of intermediates in single CAF experiment under a) nitrate reducing and b) sulfate reducing conditions showed in the chromatography spectrum. Chromatography peaks detected in the redox medium were excluded. Intermediates labelled with red rectangle were detected in mixed studies as well.

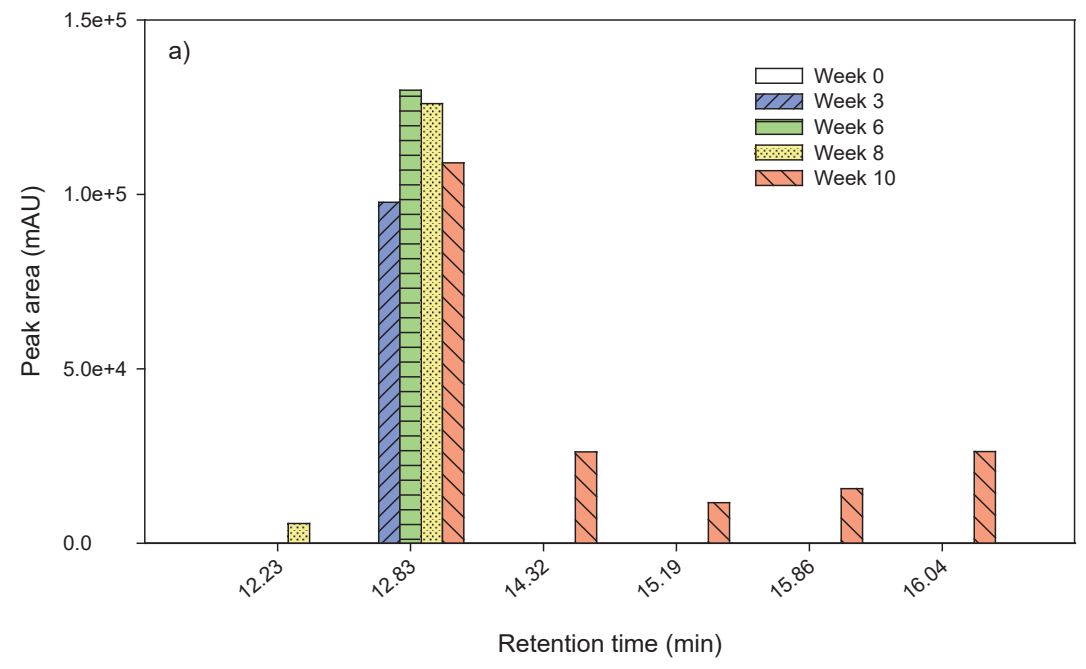



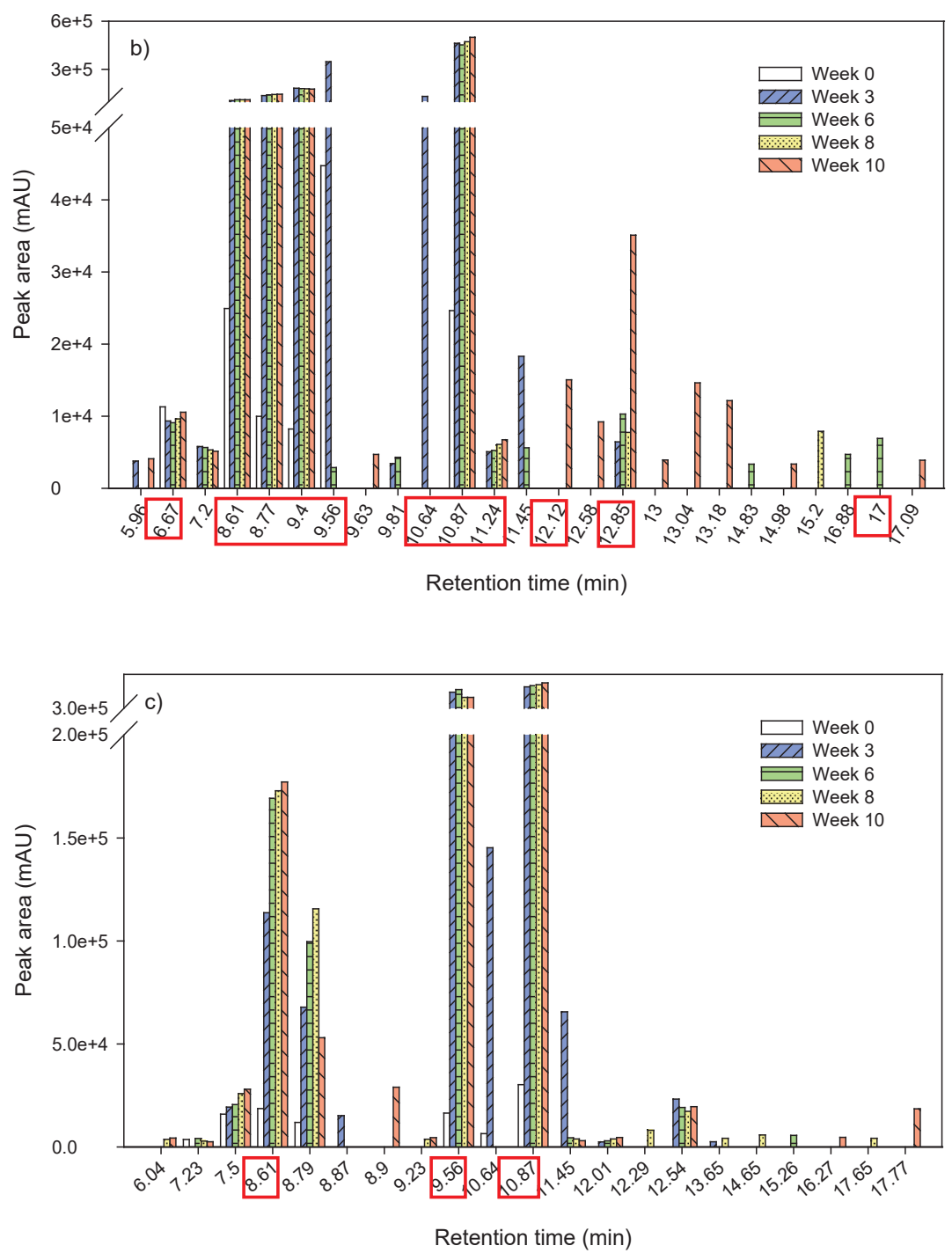

Figure S6.5 Production of intermediates in single NAP experiment under a) nitrate reducing, b) sulfate reducing and c) methanogenic conditions showed in the chromatography spectrum. Chromatography peaks detected in the redox medium were excluded. Intermediates labelled with red rectangle were detected in mixed studies as well. 

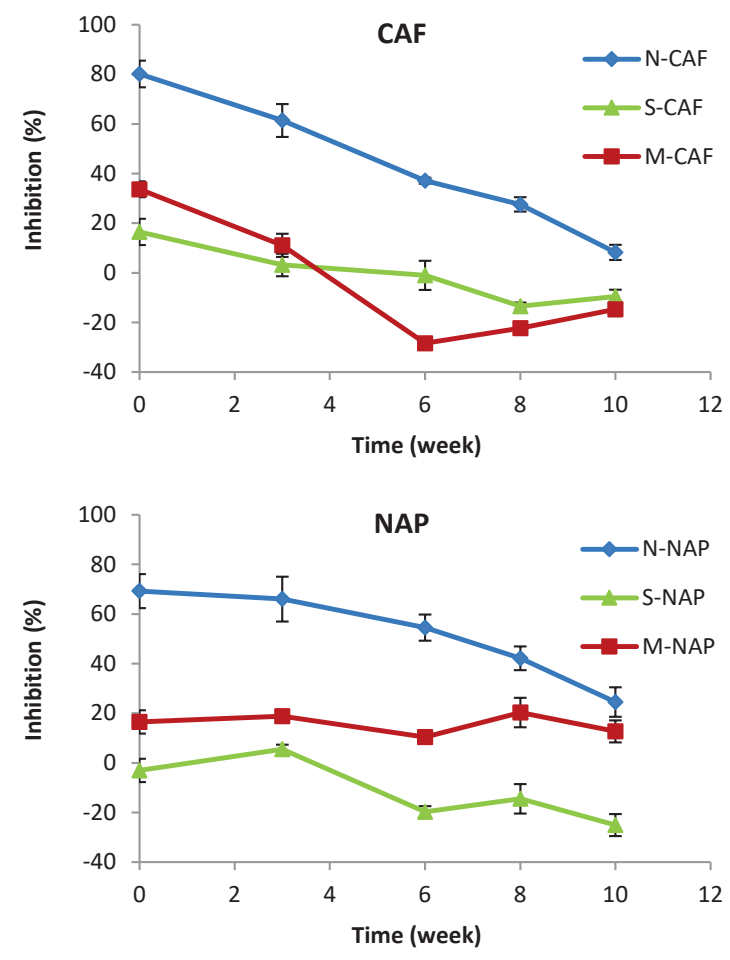

Figure S6.6 Inhibition of CAF and NAP to $V$. fischeri in three redox conditions under mixed and single status. N, S, M represent nitrate reducing, sulfate reducing and methanogenic conditions. Data are mean inhibition \pm standard error $(\mathrm{n}=3)$. 
Table S6.1 Consumption of $\mathrm{NO}_{3}{ }^{-}, \mathrm{SO}_{4}{ }^{2-}$ and $\mathrm{CO}_{2}$ after 10 weeks experiment under different investigated conditions.

\begin{tabular}{llll}
\hline $\begin{array}{l}\text { Consumption of } \\
\text { electron acceptors }\end{array}$ & Mixed PhAC group & Single CAF group & Single NAP group \\
\hline $\mathrm{NO}_{3}{ }^{-}(\mathrm{mg} / \mathrm{l})$ & 124.5 & 32.6 & 33.0 \\
$\mathrm{SO}_{4}{ }^{2-}(\mathrm{mg} / \mathrm{l})$ & 373.6 & 313.8 & 291.7 \\
$\mathrm{CO}_{2}(\mathrm{mmol} / \mathrm{l})$ & 2.1 & 2.1 & 2.3 \\
\hline
\end{tabular}

Table S6.2 Removal efficiencies of CAF and NAP in the sediment inoculum and transferred cultures.

Data are mean removal \pm standard error $(n=3)$.

\begin{tabular}{llll}
\hline \multirow{2}{*}{ Period } & Redox conditions & \multicolumn{2}{l}{ Removal efficiencies (\%) } \\
\cline { 4 - 4 } & & CAF & NAP \\
\hline Sediment batches & Nitrate reducing & 100 & 95 \\
$(3$ weeks before transfer $)$ & Sulfate reducing & 99 & 100 \\
& Methanogenic & 100 & 100 \\
\hline & Nitrate reducing & $-4.4 \pm 3.1$ & $-4.4 \pm 3.7$ \\
$1^{\text {st } \text { transfer }}$ & Sulfate reducing & $19.5 \pm 2$ & 100 \\
& Methanogenic & $2.6 \pm 3.2$ & 100 \\
\hline $\begin{array}{l}(2 \text { weeks }) \\
2^{\text {nd }} \text { transfer }\end{array}$ & Nitrate reducing & $-1.7 \pm 3$ & $-6.2 \pm 6.9$ \\
& Sulfate reducing & $8.9 \pm 2.1$ & 100 \\
\hline
\end{tabular}




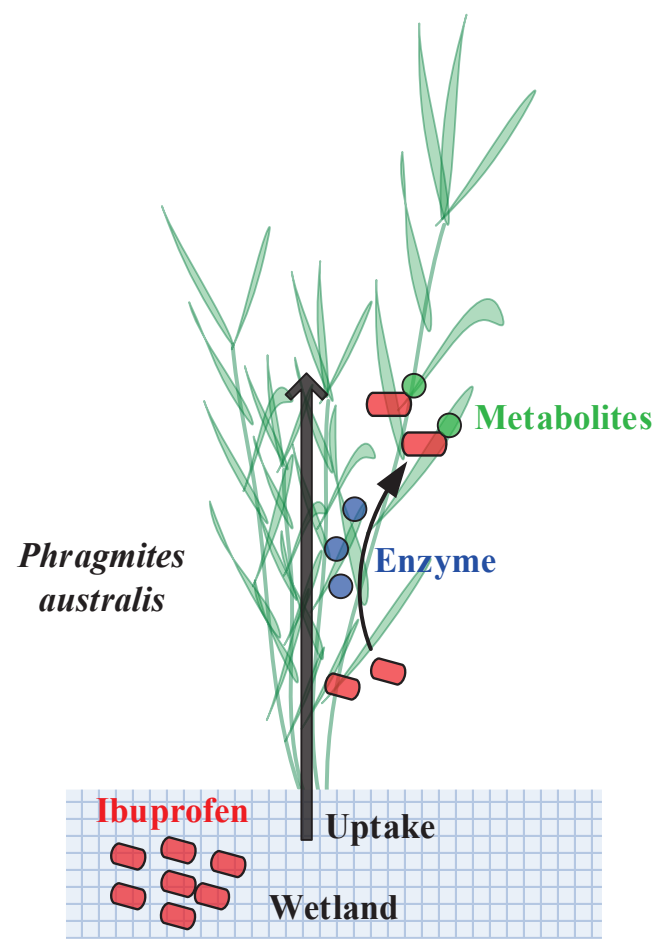




\section{Chapter 7}

\section{Metabolism of ibuprofen by Phragmites australis: uptake and phytodegradation}

A modified version of this chapter was published as

He, Y., Langenhoff, A.A.M., Sutton, N.B., Rijnaarts, H.H.M., Blokland, M.H., Chen, F., Huber, C., Schröder, P. Metabolism of ibuprofen by Phragmites australis: uptake and phytodegradation. Environmental Science \& Technology 2017, 51 (8), 4576-4584. 


\begin{abstract}
This study explores ibuprofen (IBP) uptake and transformation in the wetland plant species Phragmites australis and the underlying mechanisms. We grew $P$. australis in perlite under greenhouse conditions and treated plants with $60 \mu \mathrm{g} / \mathrm{l}$ of IBP. Roots and rhizomes (RR), stems and leaves (SL), and liquid samples were collected during 21 days of exposure. Results show that $P$. australis can take up, translocate, and degrade IBP. IBP was completely removed from the liquid medium after 21 days with a half-life of 2.1 days. IBP accumulated in RR and was partly translocated to SL. Meanwhile, four intermediates were detected in the plant tissues: hydroxy-IBP, 1,2-dihydroxy-IBP, carboxy-IBP and glucopyranosyloxyhydroxy-IBP. Cytochrome P450 monooxygenase was involved in the production of the two hydroxy intermediates. We hypothesize that transformation of IBP was first catalyzed by P450, and then by glycosyltransferase, followed by further storage or metabolism in vacuoles or cell walls. No significant phytotoxicity was observed based on relative growth of plants and stress enzyme activities. In conclusion, we demonstrated for the first time that $P$. australis degrades IBP from water and is therefore a suitable species for application in constructed wetlands to clean wastewater effluents containing IBP and possibly also other micropollutants.
\end{abstract}

\title{
Keywords
}

Ibuprofen; Phytoremediation; Plant uptake; Phytodegradation; Enzymatic activity; Phytotoxicity 


\subsection{Introduction}

In recent years, pharmaceuticals have provoked increasing concern due to their potential risks to the environment. These ubiquitous, persistent and biologically active compounds can disturb both mammalian and non-mammalian organisms [316]. Ibuprofen (IBP) is one of the most commonly used nonsteroidal anti-inflammatory pharmaceuticals with high consumption rates, for example, $4.7 \mathrm{mg} /$ inhabitant/day in China [134]. In addition, non-negligible levels of IBP have been found in water bodies (20 ng/l) and sediment (17-314 ng/g) [192, 262]. In the influents of four investigated wastewater treatment plants (WWTPs) in Spain, concentration of IBP was in the range of 12-373 $\mu \mathrm{g} / \mathrm{l}$. Following treatment, 1-48 $\mu \mathrm{g} / \mathrm{l}$ of IBP was measured in the effluents [316]. These effluent concentrations may be concerning, considering that IBP concentrations higher than $10 \mu \mathrm{g} / \mathrm{L}$ could be embryotoxic to zebrafish causing decreased hatching and growth rates [88]. Hence, IBP can be used as one of the guide-compounds to assess the treatability of waste water treatment plant effluents with respect to pharmaceutical removal [264]. In this study we focus on phytoremediation technologies for IBP removal.

Phytoremediation is the over-arching term for a group of technologies that utilize plants and the associated rhizosphere microorganisms to remove or transform contaminants leached from soils/sediments and from used water streams [339]. Plants have a pronounced ability for uptake and detoxification of many recalcitrant xenobiotics, and thus function in nature as a "green liver" [356]. A highly studied and relevant field within phytoremediation is the use of constructed wetlands (CWs) for removing pharmaceuticals from wastewater treatment plant effluents [58].

Some studies applied hydroponic plant microcosms as simplified CWs, in which uptake of IBP by the wetland plant species was confirmed. However, no further investigation into phytodegradation and underlying mechanisms was performed [102, 206, 409]. To date, only one study reported phytodegradation of IBP in duckweed by detecting intermediates in the tissue [293]. Transformation of IBP in plants may be explained by the interactions of IBP with enzymes, as plants possess a metabolic cascade capable of detoxifying xenobiotics, which resembles functions of mammalian livers [314]. This "green liver" concept points out the main enzymes playing a role in the detoxification process, include cytochrome P450 monooxygenase (P450), glycosyltransferase (GT) and glutathione-S transferase (GST) [27]. 


\section{Phytoremediation}

The aim of this study is to investigate plant uptake and phytodegradation of IBP and reveal the mechanism underlying this process. Therefore the transformation of IBP and IBP-induced enzyme defense responses were studied in Phragmites australis, which is widely applied in CWs and effectively takes up IBP, as compared with other macrophytes [206]. The results presented here prove phytodegradation of IBP via enzymes present in the wetland plant species, provide insight into the transformation mechanisms, and act as a further verification of using those plant species for phytoremediation of pharmaceuticals in CWs.

\subsection{Materials and methods}

\subsubsection{Chemicals and reagents}

IBP sodium salt ( $\geq 98 \%$ ) was purchased from Sigma-Aldrich (USA). Chemical characteristics of IBP are shown in Table S2.1 in Supporting Information (SI). Acetonitrile, formic acid, ammonium formate and water (Biosolve B.V., the Netherlands) used for liquid chromatography (LC) analysis were of LC grade. All other chemicals and reagents used in enzyme extraction and analysis of enzyme activities were of analytical grade or higher (Text S7.1). Deionized water from a Milli-Q system (Millipore, USA) was used to prepare all solutions.

\subsubsection{Experimental design}

P. australis was obtained from a local nursery (Wasserpflanzengärtnerei Jörg Petrowsky, Eschede, Germany) and transferred to pots filled with clean perlite. Plants were cultivated under greenhouse conditions for 6 weeks: relative humidity of $60 \%$ (day) and $70 \%$ (night), temperature of $22{ }^{\circ} \mathrm{C}$ (day) and $17^{\circ} \mathrm{C}$ (night), and 12 day/night hours and fed with modified Hoagland culture medium [84] step by step from $10 \%, 30 \%, 50 \%, 80 \%$ to $100 \%$ strength. Plants were illuminated with high pressure sodium lamps (Philips SON-TAGRO, $400 \mathrm{~W}$ ) with a wavelength of 400-700 nm [281]. After cultivation, plants were transferred to new pots with clean perlite, in order to minimize biodegradation of IBP by phytoplankton and microbes. Plants growth conditions are shown in Figure S7.1. During the exposure experiment, full strength medium was employed. Exposure concentration of IBP was $60 \mu \mathrm{g} / \mathrm{l}$, which is close to the concentration of wastewater effluents shown in the introduction. $500 \mathrm{ml}$ IBP solution was added to each pot with $200 \mathrm{~g}$ perlite. Batch experiments were conducted to investigate the sorption of IBP on perlite (Text S7.2). To compensate for water loss due to 
evaporation (41.4 ml/day in blank groups), $300 \mathrm{ml}$ tap water was added twice to the pots resulting in exposed water surface.

Three groups of plants were used: treated plants with $60 \mu \mathrm{g} / 1$ of IBP injection into the medium (treated groups), parallel untreated plants (untreated groups) and blank control pots without plants (blank groups). The amount of perlite was the same for all these three groups. Tissue samples were collected from both the treated and untreated groups on day $0,3,7,14$, and 21 after IBP exposure. At each sampling time point, triplicates of treated and untreated plants were sacrificed to harvest plant tissues. Harvested tissues were divided into two sections: roots and rhizomes (RR tissue), and stems and leaves (SL tissue), prior to being frozen in liquid nitrogen and stored at $-80{ }^{\circ} \mathrm{C}$ until sample processing. At the same time points, liquid samples were collected from pots of both treated and blank groups, and were filtered through $0.45 \mu \mathrm{m}$ pore size PVDF syringe filters (Carl Roth, Germany) then stored at $20{ }^{\circ} \mathrm{C}$ until analysis. Weight of plants and water loss in pots were measured prior to sampling.

\subsubsection{Selection of IBP intermediates}

According to the "green-liver" concept, plant detoxification of xenobiotics may show similarities with mammalian liver functions. Plants can metabolize xenobiotics via specific enzymatic reactions, namely (Table 7.1):

A) phase I, transformation of xenobiotics to more water soluble compounds via P450 to allow further conversion.

B) phase II, conjugation with glycosides via GT, glutathione via GST, or amino acids to reduce toxicity and alter mobility. Amino acid conjugation was reported as a side reaction rather than a main detoxification step.

C) phase III, compartmentalization of xenobiotics and metabolites into the vacuole and/or further reaction by binding to the cell wall $[25,322]$.

Some xenobiotic conjugates from phase II might still possess unwanted properties rendering them problematic for plant cells, even though they are less toxic than parent xenobiotics. Thus, it is generally accepted that those conjugates are sequestered from susceptible organs via storage in vacuoles during phase III [75]. Currently, the proposed further reactions in phase III have yet to be confirmed [344]. 


\section{Phytoremediation}

Based on metabolism principles $[78,322]$ and detected IBP metabolites in mammal and microbial systems [76, 124], we selected possible intermediates of IBP (Table 7.1).

\subsubsection{Chemical extraction}

IBP and potential intermediates in RR and SL tissue were extracted from $0.5 \mathrm{~g}$ of frozen plant tissue. Samples were ground to a fine powder in liquid nitrogen and mixed with $1 \mathrm{ml}$ of 0.1 $\mathrm{M} \mathrm{HCl} /$ acetonitrile $(50 / 50, \mathrm{v} / \mathrm{v})$ by vortexing. The mixture was then incubated on ice and mixed on a plate shaker (Neolab, Germany) for 20 min prior to centrifugation at 12,000 rpm for 15 min under $4^{\circ} \mathrm{C}$ (Eppendorf, Germany). Solid phase extraction (SPE) was performed with Oasis HLB cartridges ( $3 \mathrm{cc} / 60 \mathrm{mg}$, Waters, USA), pre-conditioned with $3 \mathrm{ml}$ methanol and equilibrated with $3 \mathrm{ml}$ deionized water. After loading $600 \mu \mathrm{L}$ sample at a flow rate of 1 $\mathrm{ml} / \mathrm{min}$, the cartridge was washed with $6 \mathrm{ml}$ deionized water and eluted with $6 \mathrm{ml}$ of $25 \%$ $\mathrm{NH}_{4} \mathrm{OH}: \mathrm{MeOH}(8 / 92, \mathrm{v} / \mathrm{v})$. Finally, the eluate was evaporated to dryness under gentle nitrogen flow (Dri-block heater, Techne, UK). The final extract was made up to exactly $1 \mathrm{ml}$ with $2 \% \mathrm{MeOH}$ by weight. The recovery of SPE method was $110-123 \%$. Validation of the extraction method is described in Text S7.3.

\subsubsection{Chemical analysis}

IBP and potential intermediates were measured by high resolution accurate mass spectrometric (HRMS) detection. The used LC-HRMS system consisted of an Ultimate 3000 LC system coupled through a HESI II electrospray source to a Q-Exactive Orbitrap MS (Thermo Fisher Scientific, San Jose, CA, USA). The LC-column used was an Atlantis HILIC Silica T3 column $(3.0 \times 100 \mathrm{~mm}, 3 \mu \mathrm{m})$ (Waters, USA). The mobile phase consisted of: eluent A, water/acetonitrile/formic acid/ammonium formate (900/100/0.02/2); and eluent B, same components with eluent A (100/900/0.02/2). Flow rate was set at $0.4 \mathrm{ml} / \mathrm{min}$. The step gradient was as follows: $0-0.5 \mathrm{~min} 10 \% \mathrm{~B}$; 0.5-6 min linearly increased to $40 \% \mathrm{~B}$; 6-7 min increased to $100 \% \mathrm{~B}$ and hold $1 \mathrm{~min} ; 8-8.1 \mathrm{~min}$ decreased to $10 \% \mathrm{~B}$ and hold until $14 \mathrm{~min}$. The column temperature was set at $60^{\circ} \mathrm{C}$ and the injection volume was $50 \mu \mathrm{l}$. Heated electrospray ion source was used for the ionization. Detection of the compounds was performed in both full scan and targeted MS/MS approach on the Q-Exactive mass spectrometer in the negative mode. Detailed operational parameters and list of target precursor ions are shown in Text S7.4 and Table S7.1. In terms of quality control, the mass calibration of the mass spectrometer was checked before analysis and re-calibrated if needed. 
External calibration was performed by calibration solution of mixed PhACs and internal calibration was performed by spiking fenoprofen as the internal standard.

\subsubsection{Enzyme extraction}

The extraction of cytochrome P450 monooxygenase (P450), glycosyltransferase (GT), glutathione-S transferase (GST), and stress enzymes including peroxidase (POX) and glutathione reductase (GR) was performed by combining methods described previously [291, 321]. During extraction, enzyme samples were kept on ice and the buffers used were precooled in the refrigerator before using, in order to maintain the integrity of enzymes. The extraction process for enzymes is shown in Figure 7.1. (1) $10 \mathrm{~g}$ of frozen plant tissue was ground in liquid nitrogen. The homogenized tissue was then mixed with $40 \mathrm{ml}$ extraction buffer and stirred at $300 \mathrm{rpm}$ for $15 \mathrm{~min}$. The extraction buffer contained $250 \mathrm{mM}$ of sucrose, $1 \mathrm{mM}$ of ethylenediaminetetraacetic acid, $40 \mathrm{mM}$ of ascorbic acid, $1 \mathrm{mM}$ of fresh phenylmethanesulfonyl fluoride and $10 \mathrm{mM}$ of fresh dithioerythritol (DTE) in $0.1 \mathrm{M}$ of sodium phosphate buffer $(\mathrm{pH}=7.4)$. The mixture was filtered by miracloth (EMD Millipore, USA) and centrifuged at $10,000 \mathrm{~g}$ for $15 \mathrm{~min}$ at $4^{\circ} \mathrm{C}$ (Beckman coulter avanti J-25 centrifuge, rotor JA-25.50, USA). The supernatant was collected into a $90 \mathrm{ml}$ ultra-centrifuge tube (polyallomer ultracrimp tube, Kendro, USA) and filled to full with buffer, which was then ultra-centrifuged at $100,000 \mathrm{~g}$ for $60 \mathrm{~min}$ at $4^{\circ} \mathrm{C}$ (Sorvall Discovery $90 \mathrm{SE}$ ultracentrifuge, rotor T-647.5, Japan). (2) After ultra-centrifugation, the pellet was collected and dissolved in $1 \mathrm{ml}$ of microsome buffer, which contained $1.4 \mathrm{mM}$ of DTE, $20 \%$ glycerin in of $0.1 \mathrm{M}$ sodium phosphate buffer $(\mathrm{pH}=7.4)$. The pellet solution was considered as microsomal $\mathrm{P} 450$ extracts and the supernatant was collected to further extract other cytosolic enzymes separately.

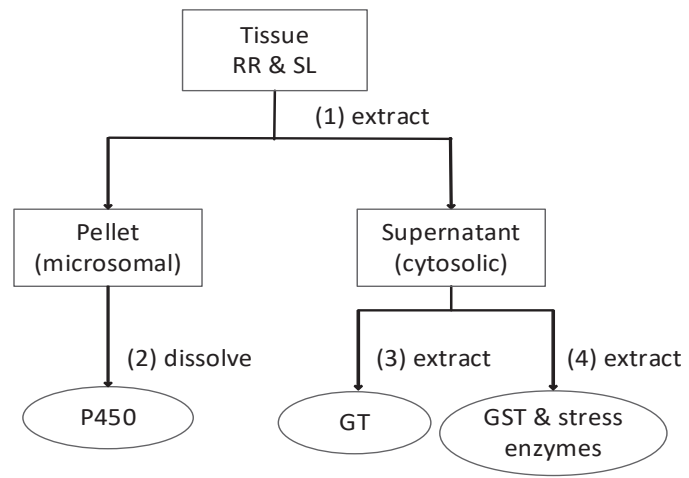

Figure 7.1 Flow chart of enzyme extraction from plant tissues. 


\section{Phytoremediation}

(3) For GT extraction, $30 \mathrm{ml}$ of the supernatant was precipitated twice by adding ammonium sulfate: first time precipitation to $40 \%$ salt saturation then centrifugation and second time to $75 \%$ saturation followed by centrifugation. The centrifugation was performed at $18,500 \mathrm{~g}$ for $30 \mathrm{~min}$ at $4^{\circ} \mathrm{C}$. The final pellet was re-dissolved in $2.5 \mathrm{ml}$ of $200 \mathrm{mM}$ Tris/ $\mathrm{HCl}$ buffer $(\mathrm{pH}=7.3)$ and subsequently desalted with PD-10 gel filtration columns (GE Healthcare, UK). (4) For extracting GST and stress enzymes, the procedure was similar to the GT protocol with a few adjustments: precipitation was applied first to $40 \%$ and second time to $80 \%$ saturation; centrifugation was conducted at $20,000 \mathrm{rpm}$; pellet was re-dissolved in $2.5 \mathrm{ml}$ of $25 \mathrm{mM}$ Tris/HCl buffer $(\mathrm{pH}=7.8)$.

\subsubsection{Determination of enzyme activity}

P450 activity was determined by an oxygen biosensor system based on the method reported by Olry et al. [285] and a commercial protocol (BD Biosciences). First, $100 \mu \mathrm{L}$ of $100 \mathrm{mM}$ Tris/ $\mathrm{HCl}$ buffer $(\mathrm{pH}=7.5), 10 \mu \mathrm{L}$ of $2 \mathrm{mM}$ cinanamic acid in ethanol, and $100 \mu \mathrm{L}$ of $\mathrm{P} 450$ extract were added into a 96-wells plate (BD ${ }^{\mathrm{TM}}$ falcon oxygen biosensor plate, USA) and incubated for $2 \mathrm{~min}$ at room temperature. The reaction was then started by adding $20 \mu \mathrm{L}$ of a regenerating solution containing $6.7 \mathrm{mM}$ glucose 6-phosphate (Glc-6-P), 0.4 units of Glc-6-P dehydrogenase, and $2 \mathrm{mM}$ nicotinamide adenine dinucleotide phosphate (NADPH) in 100 $\mathrm{mM}$ Tris buffer. The biosensor plate was then incubated in a microplate reader (Gemini EM, Modula Device, USA) to reach $27^{\circ} \mathrm{C}$. By detecting fluorescence of the oxygen sensitive dye embedded at the bottom of wells, the consumption rate of dissolved oxygen can be monitored. The fluorescence of dye $\left(\lambda_{\text {emission }}=620 \mathrm{~nm}, \lambda_{\text {excitation }}=480 \mathrm{~nm}\right)$ was recorded for 2 hours continuously in intervals of $30 \mathrm{~s}$. Wells with no addition of P450 extract were set as negative controls, while wells with $\mathrm{Na}_{2} \mathrm{~S}_{2} \mathrm{O}_{5}$ addition served as positive controls. P450 activity was calculated based on the rate of oxygen consumption.

GT activity was detected by high performance LC (Text S7.5) based on the method described by Meßner et al. [273]; POX and GR activity were measured by spectrophotometer according to methods mentioned in previous works $[320,401]$. Concentration of proteins in the extract was determined using Bradford assay [44].

Oxidative bursts might occur in plants exposed to xenobiotics [104]. The oxidative burst might increase transcription of different enzyme species or/and induce the enzymatic activity to ensure that maximum protection could be maintained in the cell compartments $[104,355]$. In this study, we used the unit nkat/mg protein to represent enzyme activities expressed in the 
extracted microsomal and cytosolic fractions, combining the possible increase of enzyme quantity and enzyme activity.

\subsubsection{Statistical analysis}

Statistical differences of enzyme activities between treated and untreated groups (at the same sampling points) were established by the analysis of variance method (ANOVA, single factor) at different significance levels. Statistical difference of IBP concentration between day 3 and 7 in blank groups was calculated in the same way. Comparisons were considered significantly different for $* \mathrm{P}<0.05, * * \mathrm{P}<0.01$ and $* * * \mathrm{P}<0.001$. The non-negligible standard deviations in this study might be caused by different growth rates and transpiration rates of parallel plants.

\subsection{Results and discussion}

\subsubsection{Uptake, accumulation and translocation of IBP in plants}

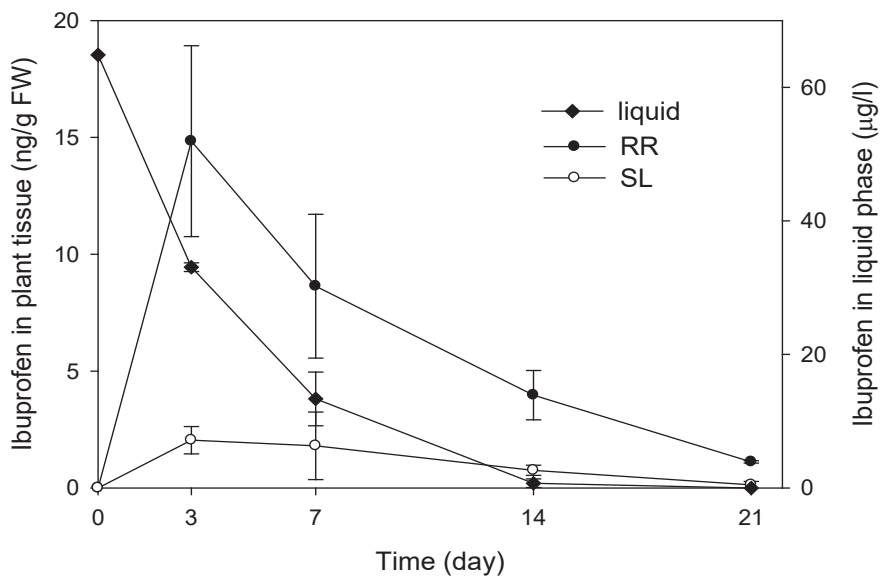

Figure 7.2 Concentration of IBP in the liquid phase and plant tissue (RR, roots and rhizomes; SL, stems and leaves) of treated groups. Data are mean concentrations (FW, fresh weight) \pm standard error $(\mathrm{n}=3)$.

In order to evaluate the uptake, accumulation and translocation of IBP in plant tissues, we investigated IBP concentrations in liquid medium and in RR and SL tissue of treated plants. IBP in the liquid phase was completely removed after 21 days of exposure (Figure 7.2). Removal followed a pseudo-first order reaction with a half-life of $t_{1 / 2}=2.1 \mathrm{~d}\left(\mathrm{R}^{2}=0.97\right)$. IBP was present in both RR and SL tissue (Figure 7.2). This indicates that IBP was transported 


\section{Phytoremediation}

upwards from medium to RR, and further translocated through RR to SL. IBP in plant tissues diminished after the initial uptake during the first 3 days (Figure 7.2). This may indicate phytodegradation. As plants lack excretory pathways for xenobiotics, they can only store those compounds in vacuoles or cell walls, or metabolize xenobiotics into non-toxic forms [26]. Thus, $P$. australis could take up, translocate and possibly degrade IBP.

To further demonstrate the capacity of $P$. australis to take up and translocate IBP, we calculated its bioconcentration factor $\left(\mathrm{BCF}_{\mathrm{RR}}, \mathrm{BCF} \mathrm{F}_{\mathrm{SL}}\right)$ and translocation factor $(\mathrm{TF})$. $\mathrm{BCF}_{\mathrm{RR}}$ and $\mathrm{BCF}_{\mathrm{SL}}$ are the ratios of IBP in RR or SL, respectively, to the spiked concentration in the medium; TF is the ratio of IBP in SL to RR, all expressed as fresh weight concentration. During the whole exposure period, $\mathrm{BCF}_{\mathrm{RR}}, \mathrm{BCF}_{\mathrm{SL}}$ and $\mathrm{TF}$ were in the range of 0.06-0.23 $\mathrm{L} / \mathrm{kg}, 0-0.03 \mathrm{~L} / \mathrm{kg}$, and $0.01-0.21$, respectively. These values are in line with IBP uptake by other aquatic plant species and vegetables in previous studies [379].

Plant uptake and translocation are thought to be strongly dependent on the physicochemical characteristics of the chemical, such as the dissociation constant $\mathrm{pKa}$ and octanol-water partition coefficient $K_{o w}$ [47]. The fate of neutral compounds in plant tissues has been frequently addressed. However, numerous uncertainties remain for ionic compounds such as IBP. IBP is acidic with a pKa of 4.91. It was partly dissociated in the growth medium, because plant exudates reduce the neutral medium $\mathrm{pH}$ to 5.2-5.7. Undissociated IBP can thus diffuse to the apoplast of root cells, and remain neutral due to the low $\mathrm{pH}$ of apoplasts $(\mathrm{pH}=$ 4-6) [274]. Thereafter, IBP may be further transferred to the cytosol, resulting in a notable $\mathrm{BCF}_{\mathrm{RR}}$. However, once inside a cell $(\mathrm{pH}=7-7.5$ in cytosol) [274], IBP was most likely ionized for more than $99 \%$ due to the elevated $\mathrm{pH}$, and trapped in the root cells. The membrane of plant cells has a negative electrical potential [274], resulting in a repulsion between membrane and negatively charged IBP and creating a barrier for diffusion of ionized IBP outside of the cytosol. We used the distribution constant $\log \mathrm{D}_{\mathrm{ow}}$, as $\mathrm{pH}$ corrected $\log$ $\mathrm{K}_{\mathrm{ow}}$, to describe the hydrophobicity of IBP $\left(\log \mathrm{K}_{\mathrm{ow}}=3.97\right)$. IBP is relatively hydrophobic with a $\log \mathrm{D}_{\mathrm{ow}}$ of 1.88 assuming a $\mathrm{pH}=7$ in a given cytosolic environment (Table S2.1). The negatively charged based repulsion and hydrophobic characteristics apparently made further transfer of IBP difficult, as observed in the low $\mathrm{BCF}_{\mathrm{SL}}$ and $\mathrm{TF}$. 


\subsubsection{Fate and transformation of IBP in the hydroponic-plant system}

\subsubsection{Metabolism of IBP in the liquid phase}

Concentrations of parent IBP and related intermediates were analyzed in the liquid medium of both treated groups with plants, and blank groups without plants. In the treated groups, an immediate exponential-wise decline of IBP concentrations was observed (Figure 7.3). In the blank groups, during the first 7 days a much smaller decline was observed, i.e. to $45 \pm 10 \mu \mathrm{g} / \mathrm{l}$. This value is in line with independent experiments showing loss of IBP by sorption to the perlite of 20\% (Figure S7.2). After day 7, a delayed but fast decline of the IBP concentrations occurred indicating a lag phase prior to biological degradation.
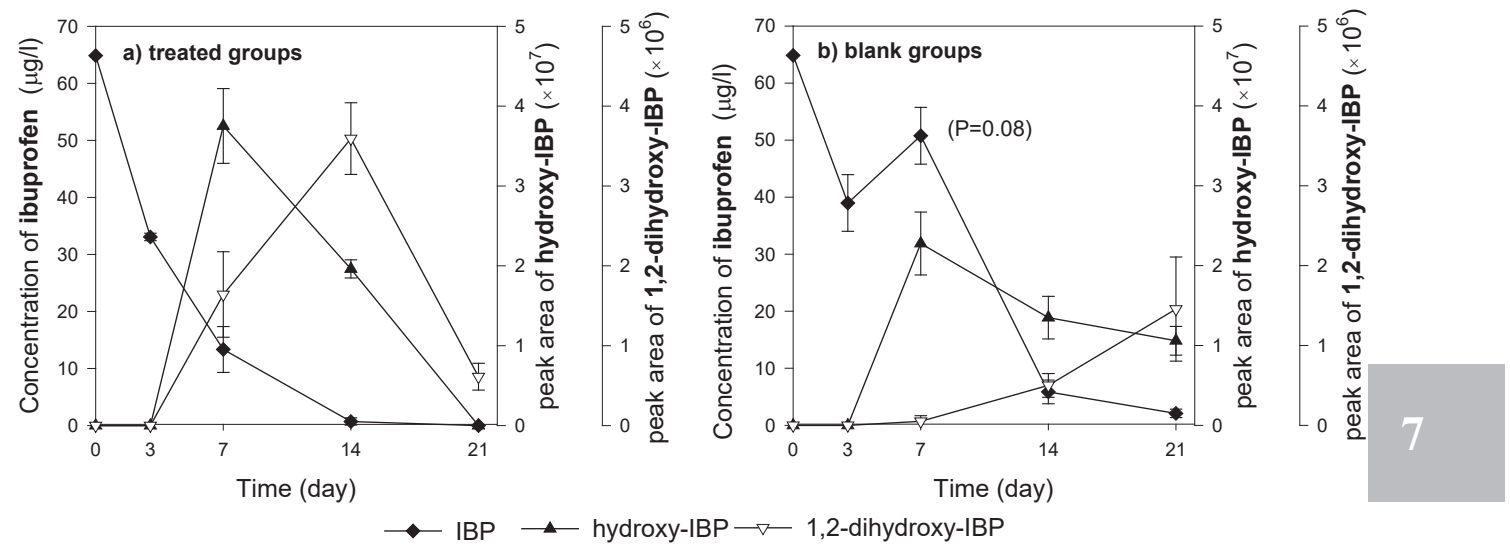

Figure 7.3 Concentration of IBP and its metabolites in the liquid phase. a) treated groups; a) blank groups without plants. Peak area of intermediates is in unit of $\mathrm{mAU} / \mathrm{ml}$ liquid. Data are mean concentrations/peak areas \pm the standard error $(n=3)$.

Hydroxy-IBP and 1,2-dihydroxy-IBP were produced in the liquid phase of both treated and blank groups (Figure 7.3), but to a much lower extent in blank groups. These hydroxy intermediates can be the result of photodegradation or biodegradation in both groups. Formation of these intermediates was indeed observed in photodegradation and biodegradation in previous research $[124,182]$. In our system, photodegradation might occur, but be less significant than biodegradation. Photodegradation of IBP was possible in the exposed water surface but most likely negligible, as our greenhouse lamps did not include UV wavelengths and the greenhouse roof filtered most sunlight UV [202]. Previous studies indicate that photodegradation of IBP by visible light would be negligible without addition of photosensitizer or catalyst $[37,184]$.In the greenhouse, fresh water phytoplankton could grow 


\section{Phytoremediation}

in the nutrient-rich medium, as well as heterotrophic microorganisms like bacteria, protozoa feeding on the algae and nutrients. Indeed, microbial biomasses including algae were found on the surface of RR tissue and perlite, which might contribute to IBP removal.

Metabolite production was more pronounced and proceeded at higher initial rates in the treated groups than in the blank groups (Figure 7.3), indicating that the presence of plants enhanced biodegradation. It is reported that roots could release exudates containing ions, inorganic and organic acids, proteins, and enzymes. Such exuded enzymes, including peroxidases and hydrolases, can catalyze the oxidation of xenobiotics [313].Furthermore, IBP has been reported to have a relatively high biodegradability [101]. Organic acids and proteins released by roots might favor rhizosphere bacteria to degrade IBP by acting as additional carbon substrates for microbial growth. Thus, the differences in intermediate production rates between treated and blank groups may be due to a combination of enzymatic reactions and enhanced rhizosphere mediated biodegradation.

\subsubsection{Metabolism of IBP in plant tissue}

Table 7.1 Metabolites of IBP in P. australis were tentatively identified by mass spectrometry.

\begin{tabular}{|c|c|c|c|c|c|c|c|}
\hline \multirow[b]{2}{*}{ Metabolism } & \multirow[b]{2}{*}{ Enzyme } & \multirow[b]{2}{*}{$\begin{array}{l}\text { Selected } \\
\text { intermediates }\end{array}$} & \multirow[b]{2}{*}{ Formula } & \multicolumn{2}{|l|}{ Tissues } & \multicolumn{2}{|c|}{ Liquid phase } \\
\hline & & & & $\begin{array}{l}\text { roots \& } \\
\text { rhizomes }\end{array}$ & $\begin{array}{l}\text { stem } \\
\& \\
\text { leave } \\
\end{array}$ & $\begin{array}{l}\text { treated } \\
\text { groups }\end{array}$ & $\begin{array}{l}\text { blank } \\
\text { groups }\end{array}$ \\
\hline \multirow{3}{*}{ phase I } & \multirow{3}{*}{ P450 } & hydroxy-IBP & $\mathrm{C}_{13} \mathrm{H}_{18} \mathrm{O}_{3}$ & $\sqrt{ }$ & $\sqrt{ }$ & $\sqrt{ }$ & $\sqrt{ }$ \\
\hline & & 1,2-dihydroxy-IBP & $\mathrm{C}_{13} \mathrm{H}_{18} \mathrm{O}_{4}$ & $\sqrt{ }$ & $\sqrt{ }$ & $\sqrt{ }$ & $\sqrt{ }$ \\
\hline & & carboxy-IBP & $\mathrm{C}_{13} \mathrm{H}_{16} \mathrm{O}_{4}$ & $\sqrt{ }$ & ND & ND & ND \\
\hline \multirow{4}{*}{ phase II } & \multirow{3}{*}{ GT } & $\begin{array}{l}\text { glucopyranosyloxy- } \\
\text { IBP }\end{array}$ & $\mathrm{C}_{19} \mathrm{H}_{28} \mathrm{O}_{7}$ & ND & ND & ND & ND \\
\hline & & $\begin{array}{l}\text { glucopyranosyloxy- } \\
\text { hydroxy-IBP }\end{array}$ & $\mathrm{C}_{19} \mathrm{H}_{28} \mathrm{O}_{8}$ & $\sqrt{ }$ & ND & ND & ND \\
\hline & & $\begin{array}{l}\text { glucopyranosyloxy- } \\
\text { carboxy-IBP }\end{array}$ & $\mathrm{C}_{19} \mathrm{H}_{27} \mathrm{O}_{10}$ & ND & ND & ND & ND \\
\hline & GST & $\begin{array}{l}\text { IBP-glutathione } \\
\text { conjugate }\end{array}$ & $\mathrm{C}_{23} \mathrm{H}_{33} \mathrm{~N}_{3} \mathrm{O}_{8} \mathrm{~S}$ & ND & ND & ND & ND \\
\hline
\end{tabular}

Referring to the selection in 2.3 of possible IBP intermediates formed in plant tissue (Table 7.1), we detected four out of the seven hypothesized intermediates. These were: hydroxy-IBP, 1,2-dihydroxy-IBP, carboxy-IBP and glucopyranosyloxy-hydroxy-IBP (IBP-glycoside 
conjugate) in RR and the first two intermediates in SL tissue. Mass spectra of detected compounds are shown in Figure S7.3.

The two intermediates of phase I (Table 7.1), hydroxy-IBP and carboxy-IBP, were reported to be formed via mammalian and microbial biodegradation of IBP. To date, this is the first study that reports production of hydroxy-IBP and carboxy-IBP in plant tissues. 1,2dihydroxy-IBP can originate from either 1-hydroxy-IBP or 2-hydroxy-IBP [250]. In our study, we observed that the production of 1,2-dihydroxy-IBP was sequential to the production of hydroxy-IBP (Figure 7.3), suggesting that 1,2-dihydroxy-IBP might be transformed from hydroxy-IBP. With regards to phase II intermediates, GT was thought to detoxify xenobiotics by acting on functional groups such as $-\mathrm{OH},-\mathrm{NH}_{2},-\mathrm{SH}$, and $-\mathrm{COOH}$ [49]. However, our study only confirmed the existence of glucopyranosyloxy-hydroxy-IBP, while no glucopyranosyloxy-carboxy-IBP was detected. In addition, GST has a preference to catalyze conjugation at electrophilic double bonds or halogen functions [358], which clearly explains why the IBP-glutathione conjugate was not detected.

Overall, the detected IBP metabolites in P. australis were similar to the metabolites found in mammals and microbes. In contrast, in the first and so far only published data on IBP metabolism in the aquatic plant Lemna gibba L., a type of duckweed, Pietrini et al. detected hydroxy-IBP, and 1,2-dihydroxy-IBP as intermediates, but no carboxy-IBP [293]. They suggested that the metabolic pathway of IBP in duckweed was different from mammals and microbes, but similar to fungi.

Concentrations of hydroxy-IBP and 1,2-dihydroxy-IBP were 3-4 times higher in RR than in SL tissue (Figure 7.4A). Those two intermediates were also produced in the liquid phase of treated groups (Figure 7.3A). Therefore, we could not distinguish whether their presence in tissue is a result of transport from the liquid phase or production in the plant. However, we could conclude that phytodegradation did contribute to the production of hydroxy-IBP and 1,2-dihydroxy-IBP, because IBP was transformed in both RR and SL tissue (Figure 7.2). The transformation tendency of IBP showed first a boost followed by a decay, which was similar with the production of two intermediates. Additionally, the production of these two intermediates occurred sequentially to the transformation of IBP. Recent research on diclofenac (DFC) metabolism in Typha latifolia detected parent DFC, hydroxy-DFC, glucopyranosyloxy-hydroxy-DFC, and DFC-glutathione conjugate in roots, and only DFC and hydroxy-DFC in leaves [27]. In comparison, we also found for IBP the parent compound 


\section{Phytoremediation}

and hydroxy intermediates in SL, while in RR we did not detect IBP-glutathione conjugate but the carboxy-IBP. Overall, the results demonstrate that phytodegradation of IBP indeed occurred inside the tissues of $P$. australis by transforming IBP into four intermediates.
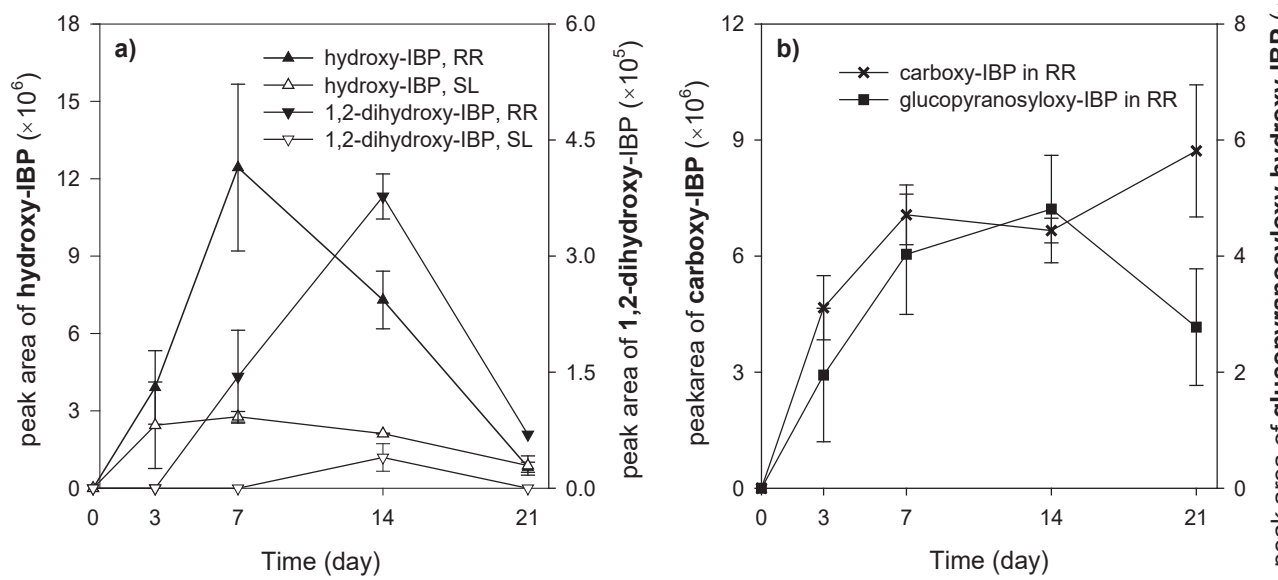

Figure 7.4 Metabolism of IBP in the plant tissue (RR, roots and rhizomes; SL, stems and leaves). a) hydroxy-IBP and 1,2-dihydroxy-IBP; b) carboxy-IBP and glucopyranosyloxy-hydroxy-IBP. Peak area of intermediates is in unit of mAU/g tissue. Data are mean peak areas \pm standard error $(n=3)$.

\subsubsection{Enzymatic activity}

In order to understand the mechanism by which IBP is transformed, we measured activities of P450, GT, and GST in tissue of treated groups and compared with those of untreated groups. In phase I and II of plant detoxification, P450, GT, and GST normally catalyze hydrolysis, oxidation and synthesis reactions [78]. P450 activity in treated RR tissue was higher than untreated tissue after 3 days of IBP exposure and the higher trend lasted until day 14 (Figure 7.5A). The occurrence of two hydroxy intermediates appeared to correlate with the trend of higher P450 activity. In addition, a similarly pronounced correlation was found in SL tissue: increase of P450 activity was synchronous with the detection of hydroxy-IBP (Figure 7.5B). P450 activity could be interpreted as an indicator of the phase I metabolism of IBP in $P$. australis. GT activity in RR and SL tissue showed differences compared with untreated groups, but no clear link between the alteration of enzyme activity and occurrence of glucopyranosyloxy-hydroxy-IBP was found (Figure S7.4). During exposure, GST activity increased at day 3 and 7 in RR tissue of treated groups compared with that in untreated groups (Figure S7.4). However, the activity peak disappeared afterwards, and IBP- 
glutathione conjugate was not detected in RR tissue. Hence the increase may be attributed to some response to the oxidative burst condition of the tissue. In summary, our results show that P450 was involved in the production of two hydroxy intermediates; activity variation of GT and GST did not display a clear pattern that related with phytodegradation of IBP.

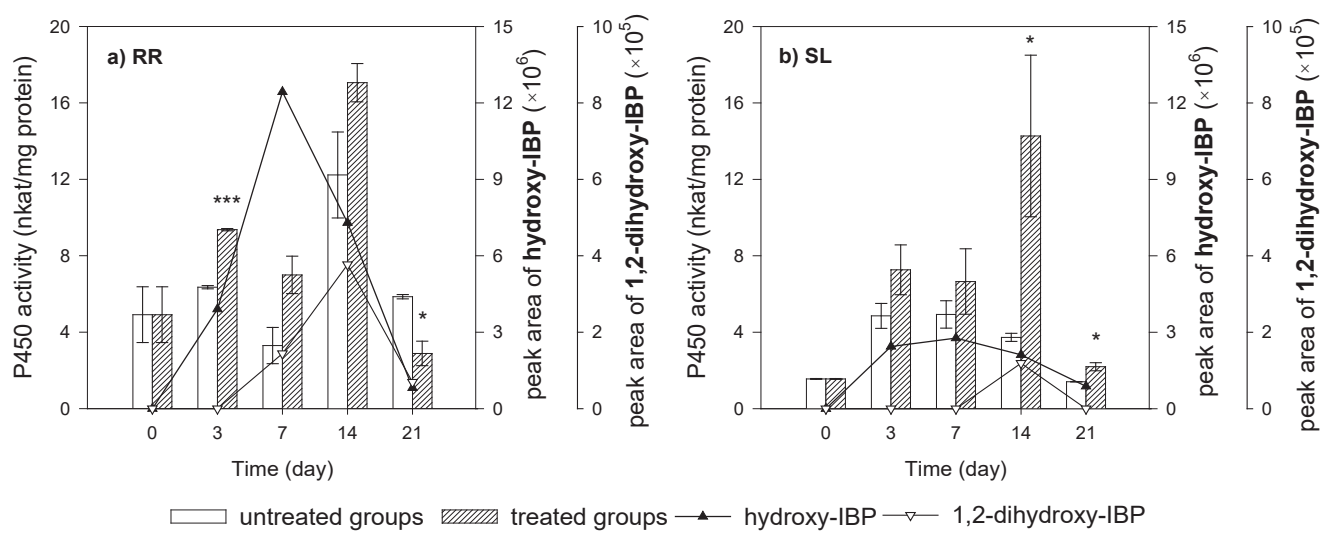

Figure 7.5 P450 activity and its relationship with production of related intermediates a) RR, roots and rhizomes b) SL, stems and leaves. Peak area of intermediates is in unit of mAU/g tissue. Data are mean activity/peak areas \pm standard error $(n=3)$.

\subsubsection{Phytotoxicity of IBP exposure}

Phytotoxicity of pollutants can affect the phytoremediation process by making plant cells more susceptible to diseases and stress conditions [58]. To determine the potential IBPinduced phytotoxicity, relative growth of plants $(\mathrm{g} / \mathrm{d})$ and activity of stress enzymes including POX and GR in RR and SL were investigated. The relative growth of P. australis showed that the biomass of all plants decreased during the first 3 days regardless of treatment, indicating plants may need an adjustment period to get used to a new growth environment and substrate (Figure S7.5). However, after 21 days exposure, relative growth of treated plants showed a similar level with that of untreated groups. Therefore, plants show resiliency during IBP exposure, as they continue to grow normally during the uptake and accumulation process and also degrade IBP with the involvement of P450.

The oxidative burst induced by xenobiotic exposure results in an over-production of reactive oxygen species (ROS) such as superoxide radicals, which can damage plant cells [104]. A stress enzyme system is one of the protective mechanisms for plants to eliminate the ROS excess [104]. In our study, no obvious difference of stress enzyme activities was observed 


\section{Phytoremediation}

between treated and untreated groups (Figure S7.6), except for the difference found on day 3 in both RR and SL. This exception might result from the growth adaptation on day 3 . The overall absence of stress enzymes might be related to the resilience of the plant species $P$. australis, or to the chosen IBP exposure concentration. Pawłowska et al. found that spring barley and common radish showed different sensitivity to the exposure of quaternary ammonium salts by showing different levels of stress enzyme activity [288]. Different exposure concentrations also affect the variation of enzyme activity. POX activity of barley turned out to have a positive linear correlation with exposure concentrations [288]. Another recent research showed that low concentrations of carbamazepine $(<6 \mu \mathrm{g} / \mathrm{l})$ stimulated the stress enzyme activity in two microalgae species. However, enzyme activity decreased at higher carbamazepine concentrations, because over-loading stress brought functional damage to microalgae cells [131]. In summary, no obvious alteration of growth rate and stress enzyme activity was observed, indicating that $P$. australis was resilient and resistant to IBP exposure.

\subsubsection{IBP transformation pathways in plant tissue}

This study shows that $P$. australis is able to take up, accumulate, and metabolize IBP, in both RR and SL tissue without significant phytotoxicity. Based on our results, we propose the following pathway for IBP metabolism: transformation of IBP was catalyzed by P450 in the endoplasmic reticulum, then catalyzed by GT in Golgi, followed by further metabolism or storage in vacuoles or cell walls (Figure 7.6).

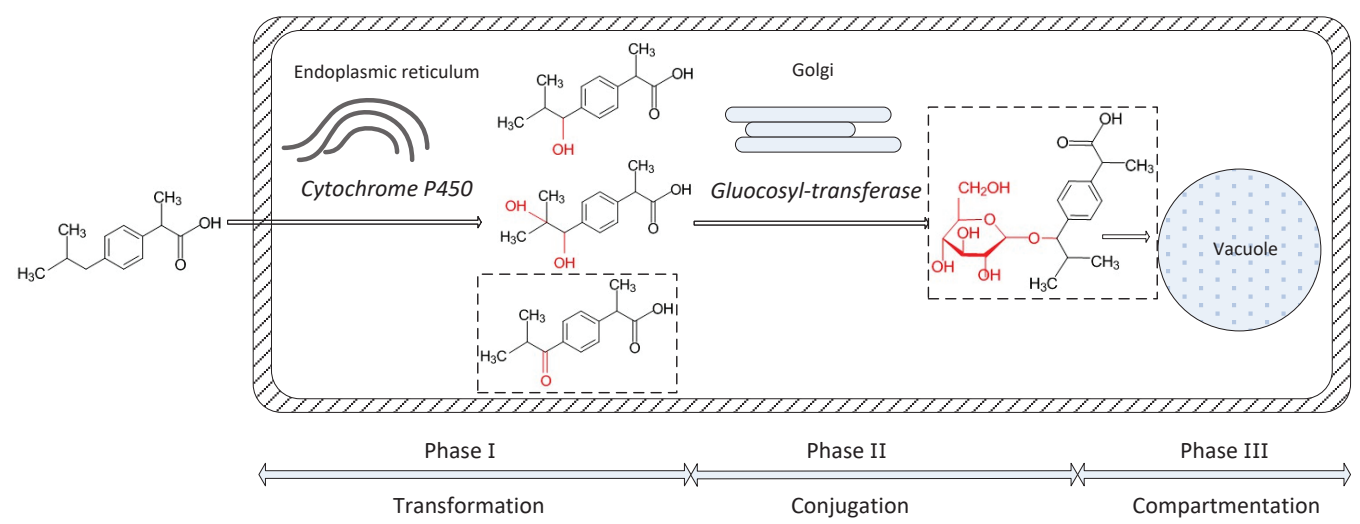

Figure 7.6 Transformation of IBP in plant tissues. 1-hydroxy-IBP was used to represent hydroxy-IBP. Carboxy-IBP and glucopyranosyloxy-hydroxy-IBP (marked with dashed rectangles) were not detected in SL tissue. 


\subsubsection{Implication for practice}

Metabolism and detoxification of IBP in mammals are known processes and have been well described. The fate and transformation of IBP in aquatic macrophytes has not been investigated so far. However, aquatic macrophytes are increasingly exposed to residual pharmaceuticals in water bodies, especially when those plants are applied in phytoremediation. Therefore, it is necessary to study the fate of pharmaceuticals in macrophytes and the underlying phytoremediation mechanism. In summary, this study gives insight on the fate and transformation of IBP in P. australis, which can be applied for investigating other pharmaceuticals and forecasting their fate in other types of macrophytes. Reflecting on practice, this study proves that macrophytes have the potential to take up and degrade pharmaceuticals. The knowledge contributes to understanding and implementing phytoremediation in constructed wetlands as an effective treatment method for removing pharmaceuticals from water.

\section{Acknowledgement}

Part of this work was carried out within a Short Term Scientific Mission (STSM) of the Water2020 Cost Action ES1202: Conceiving Wastewater Treatment in 2020 | Energetic, environmental and economic challenges. Authors thanks Rudolf Harpaintner, Andres Sauvetre and Paula Haury for technical assistance. The support provided by China Scholarship Council (CSC) for the research of Yujie He at Wageningen University is kindly acknowledged. 


\section{Phytoremediation}

\section{Supplementary materials}

Text S7.1 Chemicals and reagents

1) Chemicals used in enzyme extraction

Sucrose (Sigma), Ethylenediaminetetraacetic acid (EDTA, Sigma), ascorbic acid (Fluka), phenylmethanesulfonyl fluoride (PMSF, Fluka), Dithioerythritol (DTE, Roth), glycerine (Roth), sodium phosphate (Roth), $\mathrm{MgCl}_{2}$ (Roth), polyvenylpyrrolidone PVP K90 (Fluka), polyvenylpyrrolidone PVP K30 (Fluka), Tris(hydroxymethyl) aminomethan (Merck), nonidet P40 substitude (Fluka).

2) Chemicals used in enzyme activity analysis

Cinanamic acid (Fluka), glucose 6-phosphate (Fluka), glucose-6-phosphat dehydrogenase (Simga), nicotinamide adenine dinucleotide phosphate (NADPH, Fluka), uridine-5'diphosphoglucose (Fluka), nitrophenyl-ß-D-glucopranoside (4-PNG, Fluka), D-(-)-salicin (Fluka), quercetin dihydrat 98\% (Fluka), chlor(1-) 2,4 dinitrobenzol (CDNB, Merck), glutathion reduced (GSH, Fluka), guajacol (Fluka), hydrogen peroxide solution (35\%, Sigma), glutathion oxidized (GSSG, Fluka).

Text S7.2 Batch experiments of IBP sorption on perlite

Batch experiments were conducted to investigate the IBP sorption on perlite in triplicate.

In batch, $100 \mu \mathrm{g} / 1 \mathrm{IBP}$ solution was added to perlite in flasks as the same liquid/perlite ratio ( $200 \mathrm{~g}$ perlite $/ 500 \mathrm{ml}$ solution) in the exposure experiment. Flasks were shaken at $100 \mathrm{rpm}$ at room temperature for 8 days. To explore the desorption potential, on day 3 IBP solution was replaced with deionized water to mimic IBP removal by other mechanisms (e.g. biodegradation) in blank groups. Results are shown in Figure S7.2.

Text S7.3 Validation of IBP extraction from plant tissue

Roots and rhizomes (RR tissue, $5 \mathrm{~g}$ ), stems and leaves (SL tissue, $5 \mathrm{~g}$ ) were cut into pieces and cultivated in 100 times diluted Murashige and Skoog medium spiked with a known amount of IBP $(20 \mathrm{mg} / \mathrm{l})$. Cultivation bottles were vacuumed so that air bubbles could not block transpiration channels of tissue. After $24 \mathrm{~h}$ cultivation, medium samples were collected. Tissue were washed with demi water and frozen with liquid nitrogen. Tissue samples were then extracted according to the method described in section 2.3. Recovery of extraction was 
calculated by the ration of IBP mass extracted from tissue to IBP loss in medium. Experiments were performed in triplicate. Recovery of the extraction method was $110-123 \%$.

Text S7.4 Q-Exactive method for chemical analysis

HESI (Heated Electrospray) ion source was used for the ionization. The HESI parameters were optimized as follows: sheath gas flow rate 53 units; aux. gas flow rate 14 units; sweep gas flow rate 3 units; spray voltage $2.5 \mathrm{kV}$; capillary temperature $269{ }^{\circ} \mathrm{C}$; aux gas heater temperature $438{ }^{\circ} \mathrm{C}$; and $\mathrm{S}$ lens RF level 50. Detection of the compounds was performed using a Q-Exactive mass spectrometer. Full scan data in both positive and negative was acquired at a resolution of $17500 \mathrm{FWHM}$ (full width half maximum at $\mathrm{m} / \mathrm{z} 200$ ). For the compounds of interest, a scan range of $\mathrm{m} / \mathrm{z}$ 100-600 was chosen; the automatic gain control (AGC) was set at $3 e 6$ and the injection time was set to $200 \mathrm{~ms}$. Scan-rate was set at $1 \mathrm{scan} / \mathrm{s}$. For confirmatory purpose, a targeted MS/MS analysis was performed using the mass inclusion list. List of target precursor ions is shown in Table S7.1. The Orbitrap spectrometer was operated in negative mode at 17,500 FWHM. The AGC target was set to 2e5, with the maximum injection time of $100 \mathrm{~ms}$. The precursor ions are filtered by the quadrupole which operates at an isolation window of $\mathrm{m} / \mathrm{z} 4$. Collision energy was optimized at $20 \%$ NCE (normalized collision energy) by injected working mix standard solution at a concentration of $10 \mu \mathrm{g} / \mathrm{l}$.

Text S7.5 HPLC method for GT enzyme activity

First, $10 \mu \mathrm{l}$ of $0.1 \mathrm{mM}$ quercetin (substrate) was incubated with $20 \mu \mathrm{l}$ of $2 \mathrm{mM}$ uridine diphosphate glucose, $10 \mu \mathrm{l}$ of $3.125 \mathrm{mM} \mathrm{N}$-nitrophenyl-d-glucopyranoside and $10 \mu \mathrm{l}$ of 3.125 $\mathrm{mM}$ salicin in $50 \mu \mathrm{l}$ of $200 \mathrm{mM}$ Tris/HCl buffer ( $\mathrm{pH}$ 7.3). Second, the reaction was initiated by adding $100 \mu \mathrm{l} \mathrm{GT}$ enzyme extract. After 30 min incubation at $30{ }^{\circ} \mathrm{C}$ the reaction was stopped by protein-precipitation with $10 \mu \mathrm{l}$ of $\mathrm{H}_{3} \mathrm{PO}_{4}$ and centrifuged at 15,000 $\times \mathrm{g}$ for $2 \mathrm{~min}$. Finally, the supernatant was diluted 1:4 (v/v) with solvent A (water, $0.1 \%$ trifluoroacetic acid, TFA) of HPLC before analysis. Reverse phase HPLC was performed using a Varian Pro-Star M215 HPLC system and a C18 Prontosil Spheribond column (Bischoff Chromatography, Leonberg). Mobile phases consisted of $0.1 \%$ TFA (solvent A) and acetonitrile with $0.1 \%$ TFA (solvent B) run at a constant flow rate of $0.85 \mathrm{ml} \mathrm{min}^{-1}$ : 0-0.5 $\min 10 \% \mathrm{~B} ; 0-8 \mathrm{~min}$ linear increased from $8 \%$ to $40 \% \mathrm{~B}$ and hold $1.5 \mathrm{~min}$; $9.5-12.5$ min decreased to $8 \% \mathrm{~B}$ and hold until $15 \mathrm{~min}$. The injection volume was $20 \mu \mathrm{l}$. 


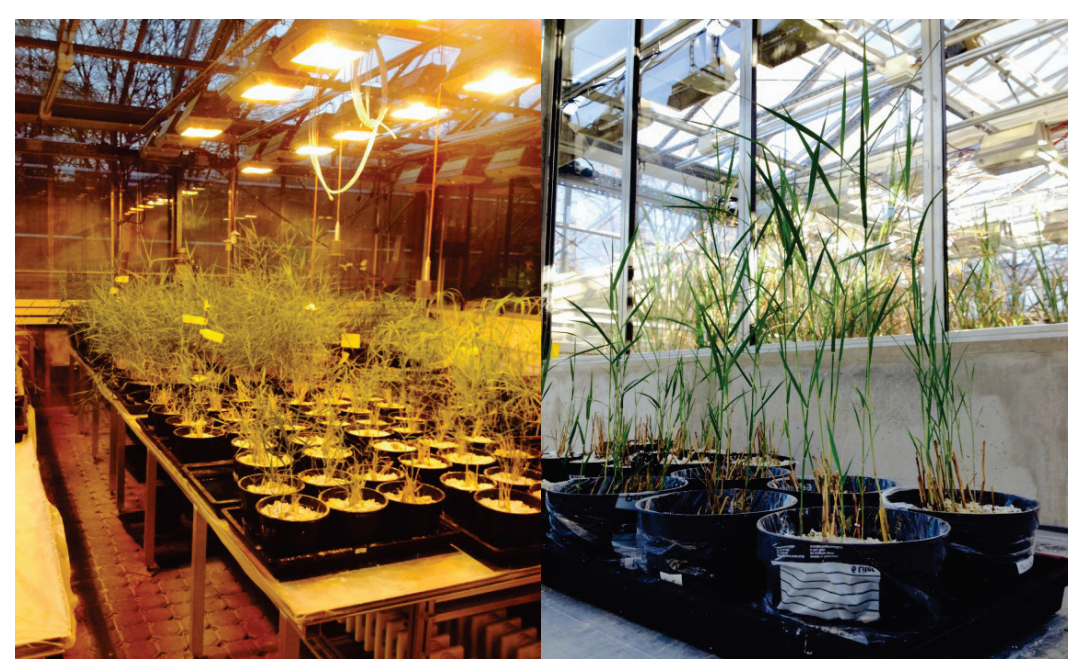

Figure S7.1. Growing plants under greenhouse conditions.

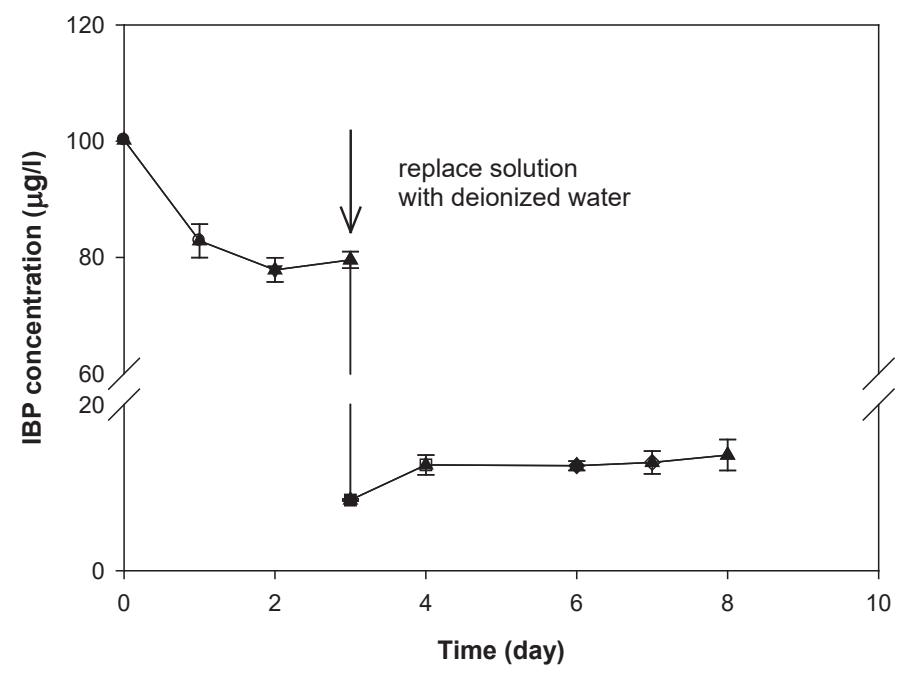

Figure S7.2 Sorption of IBP on perlite Sorption of IBP on perlite. At day 3, IBP solution was replaced with deionized water to mimic IBP removal by other mechanisms (e.g. biodegradation) in blank groups. After 3 days sorption, IBP sorption reached equilibrium. The results showed that $20 \%$ of IBP was absorbed on perlite until day 3 and desorption occurred after solution replacement. 


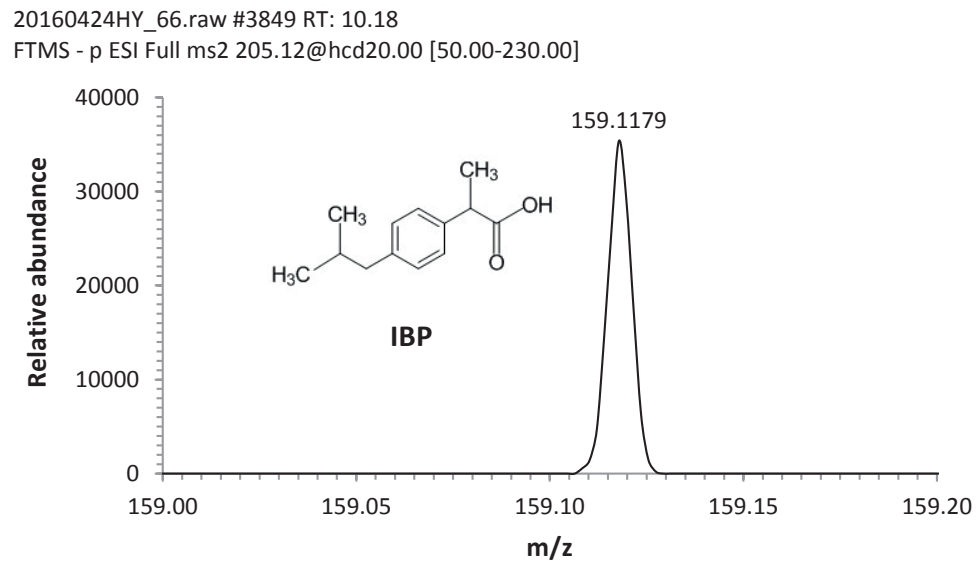

20160424HY_66.raw \#3547 RT: 9.52

FTMS - p ESI Full ms2 221.12@hcd20.00 [50.00-245.00]]

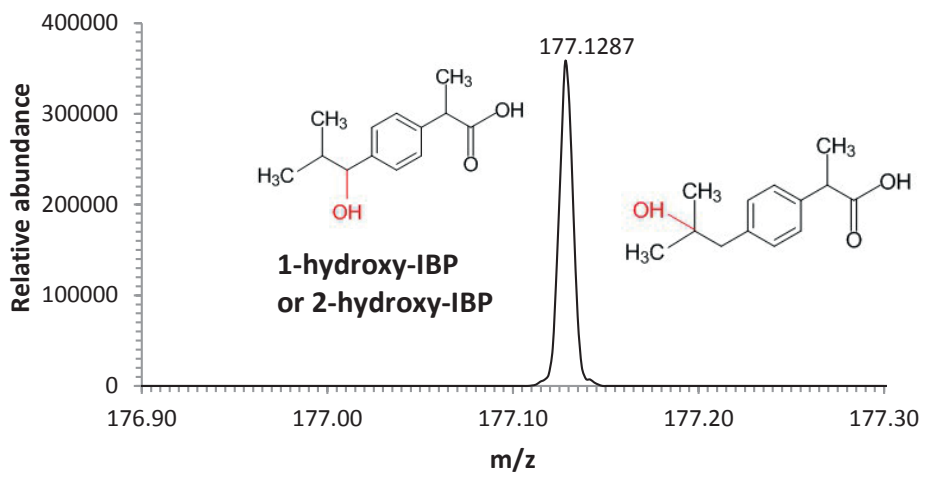


Phytoremediation

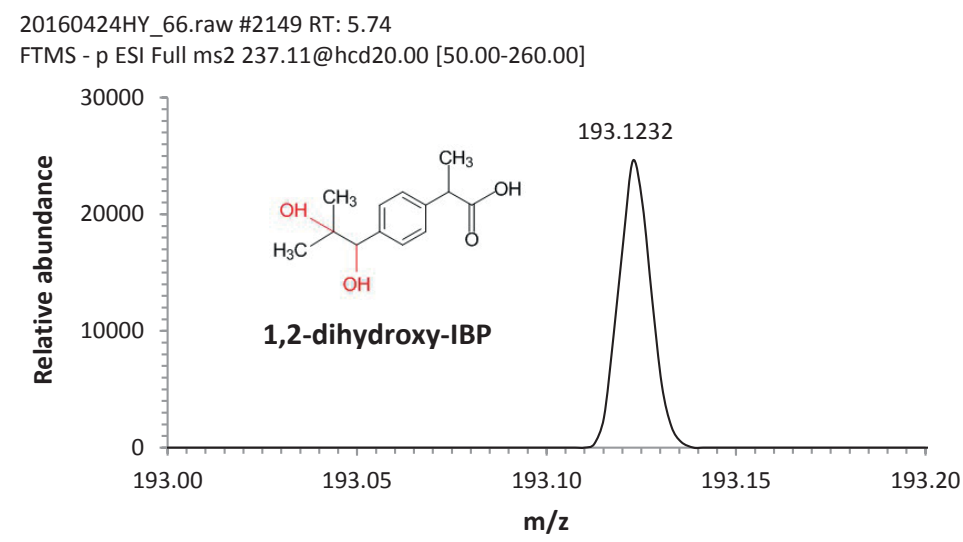

20160424HY_66.raw \#3460 RT: 9.32

FTMS - p ESI Full ms [100.00-600.00]

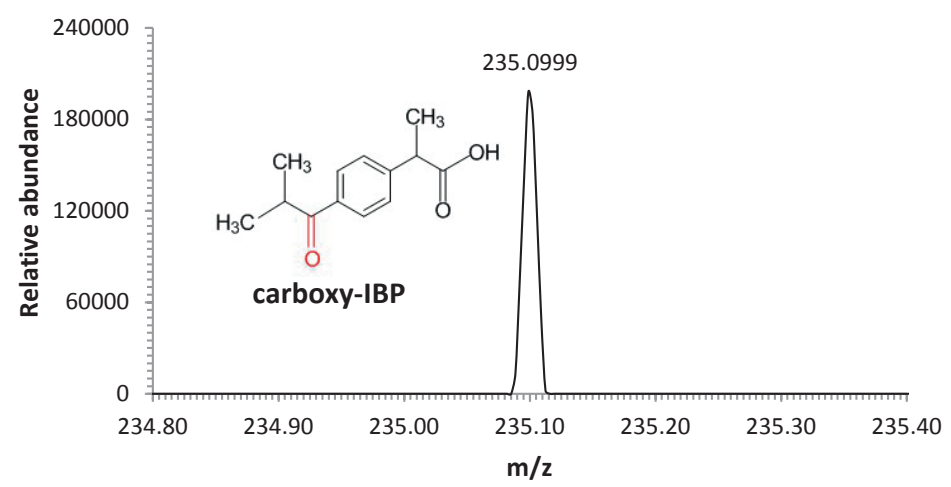

20160424HY_66.raw \#2438 RT: 6.56

FTMS - p ESI Full ms [100.00-600.00]

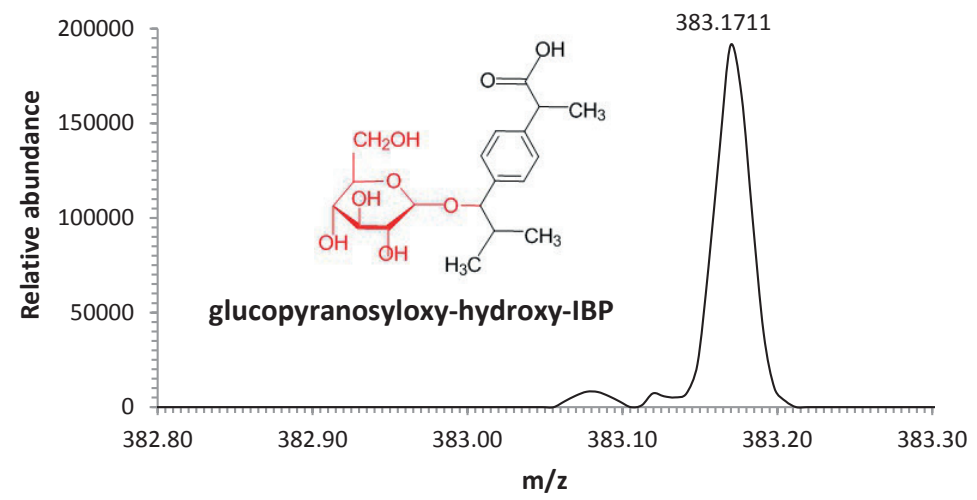

Figure S7.3 Major product ions of parent ibuprofen and related metabolites which were used to tentative identifictaion. 

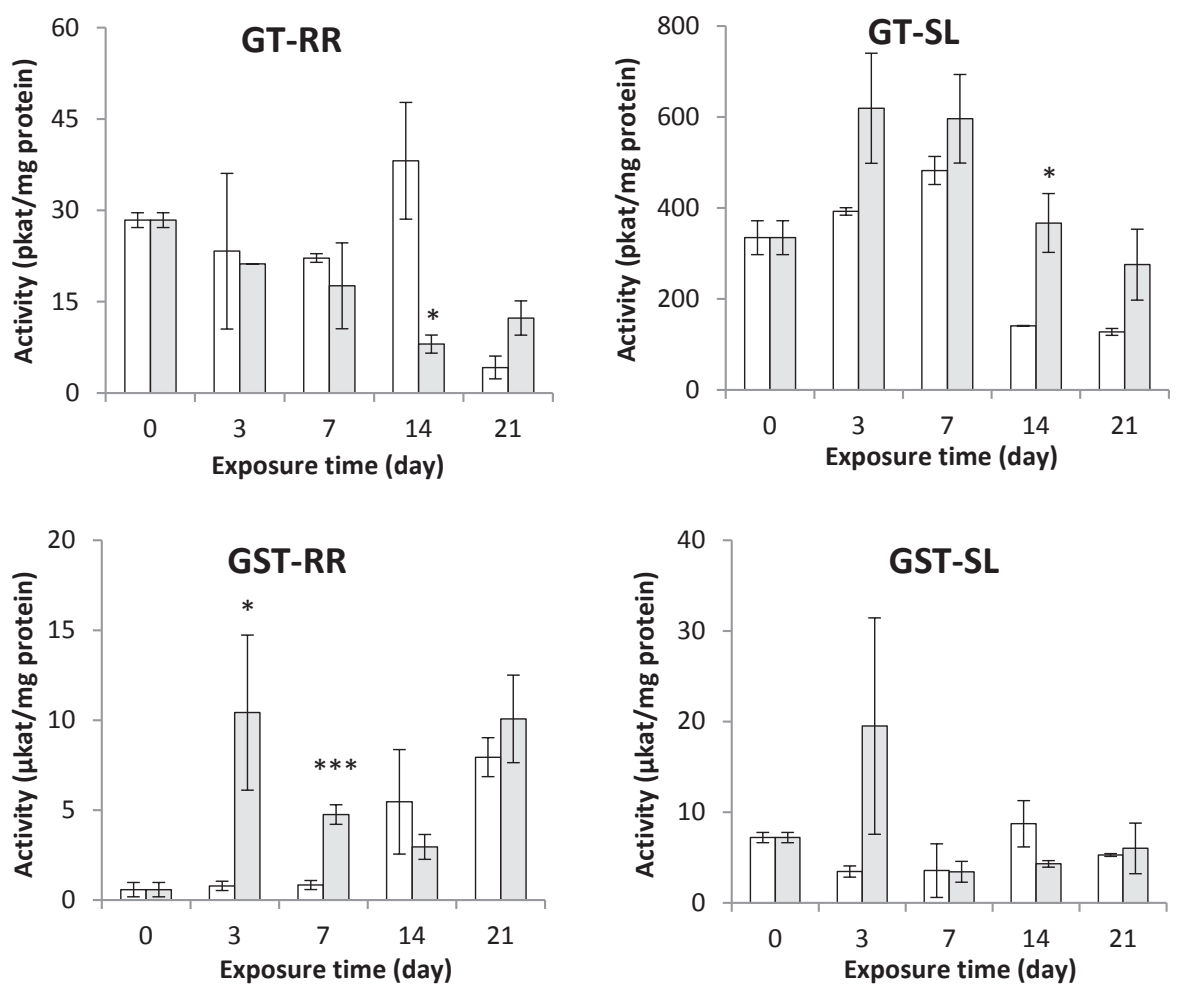

$\square$ untreated groups

\section{$\square$ treated groups}

Figure S7.4 Enzyme activities of GT and GST in plant tissues (RR, roots and rhizomes; SL, stems and leaves $)$ in untreated and treated groups. Data are mean activity \pm standard error $(n=3)$. 


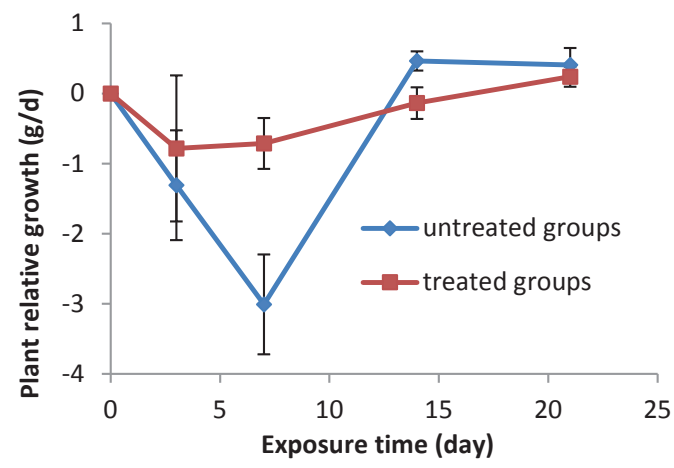

Figure S7.5 Relative growth rates of plants during the exposure period. Data are mean growth \pm standard error $(\mathrm{n}=3)$.
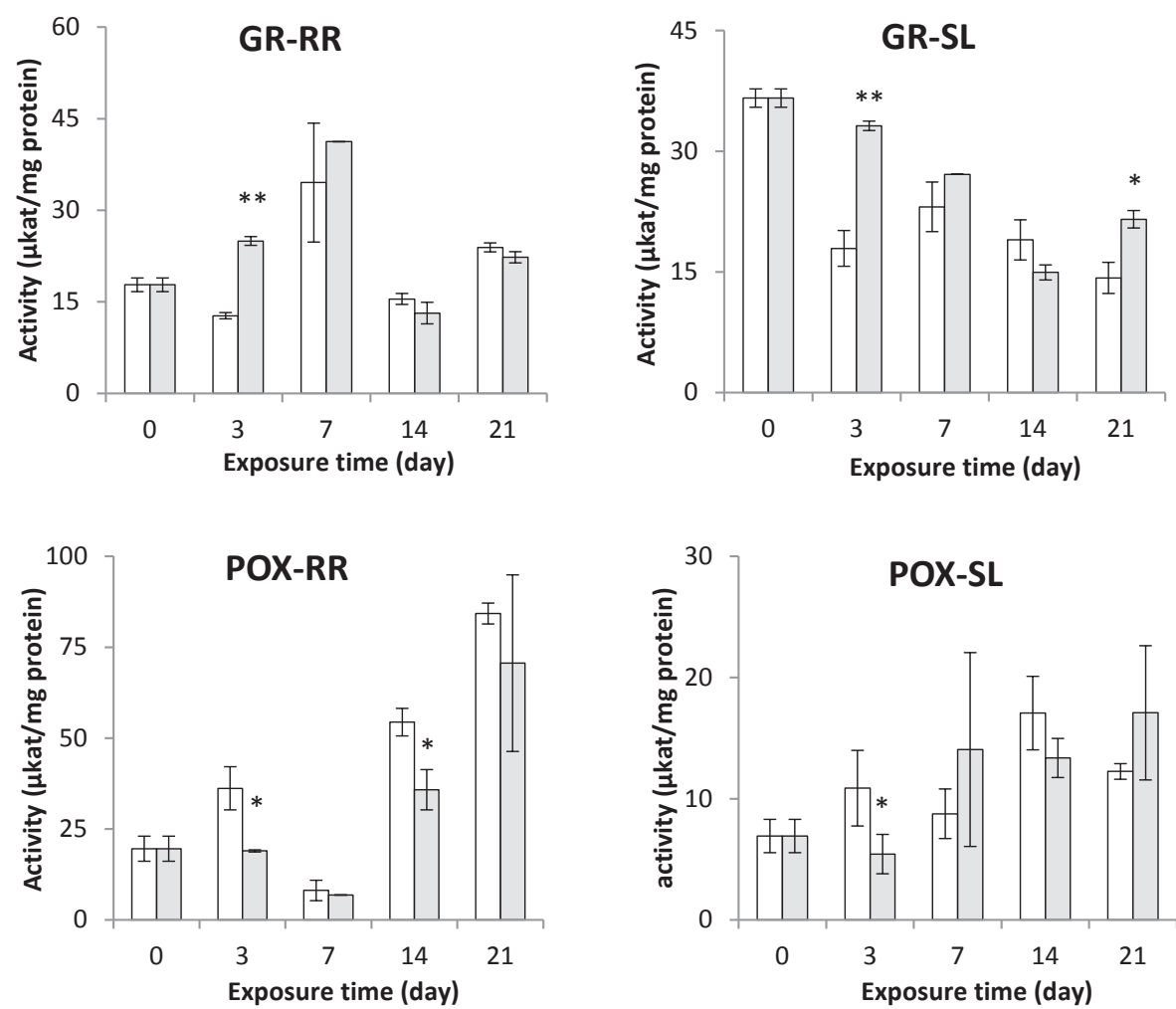

$\square$ untreated groups

$\square$ treated groups

Figure S7.6 Stress enzyme activities (POX, GR) in plant tissues (RR, roots and rhizomes; SL, stems and leaves $)$ in untreated and treated groups. Data are mean activity \pm standard error $(n=3)$. 
Table S7.1 List of target precursor ions of parent ibuprofen and related metabolites.

\begin{tabular}{|c|c|c|c|c|}
\hline Compounds & Formula & $\begin{array}{l}\text { Mass } \\
(\mathrm{M}-\mathrm{H})^{-}\end{array}$ & $\begin{array}{l}\text { Product ion } \\
(\mathrm{M}-\mathrm{H})^{-}\end{array}$ & $\mathrm{RT}(\min )$ \\
\hline IBP & $\mathrm{C}_{13} \mathrm{H}_{18} \mathrm{O}_{2}$ & 205.1234 & 159.1179 & 10.18 \\
\hline hydroxy-IBP & $\mathrm{C}_{13} \mathrm{H}_{18} \mathrm{O}_{3}$ & 221.1183 & 177.1287 & 9.52 \\
\hline 1,2-dihydroxy-IBP & $\mathrm{C}_{13} \mathrm{H}_{18} \mathrm{O}_{4}$ & 237.1132 & 193.1232 & 5.74 \\
\hline carboxy-IBP & $\mathrm{C}_{13} \mathrm{H}_{16} \mathrm{O}_{4}$ & 235.0976 & NM & 9.32 \\
\hline glucopyranosyloxy-IBP & $\mathrm{C}_{19} \mathrm{H}_{28} \mathrm{O}_{7}$ & 367.1762 & ND & ND \\
\hline glucopyranosyloxy-hydroxy-IBP & $\mathrm{C}_{19} \mathrm{H}_{28} \mathrm{O}_{8}$ & 383.1711 & NM & 6.56 \\
\hline glucopyranosyloxy-carboxy-IBP & $\mathrm{C}_{19} \mathrm{H}_{27} \mathrm{O}_{10}$ & 414.1532 & ND & ND \\
\hline IBP-glutathione conjugate & $\mathrm{C}_{23} \mathrm{H}_{33} \mathrm{~N}_{3} \mathrm{O}_{8} \mathrm{~S}$ & 510.1916 & ND & ND \\
\hline
\end{tabular}

Notes: ND means not detected; NM means not measured. 


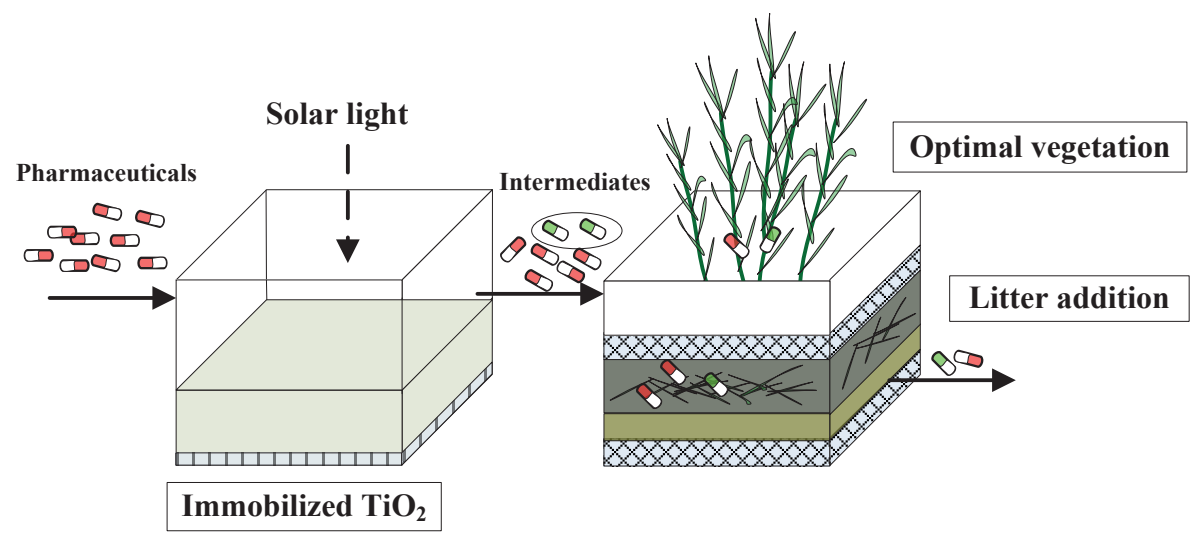




\section{Chapter 8}

\section{Fate and distribution of pharmaceuticals in mesocosm constructed wetlands}

A version of this chapter has been submitted as

He, Y., Sutton, N.B., Lei Y., Rijnaarts, H.H.M., Langenhoff, A.A.M. Fate and distribution of pharmaceuticals in mesocosm constructed wetlands. Water Research. 


\begin{abstract}
Removal of pharmaceutically active compounds (PhACs) in constructed wetlands (CWs) is a complex interplay of different processes, mainly photodegradation, sorption, biodegradation, and phytoremediation. In this research, we studied the fate and distribution of seven PhACs in mesocosm CWs and the effect of irradiation via pre-photocatalysis, substrate composition (mainly sediment) through addition of litter (dead plant biomass), and plants.
\end{abstract}

First of all, results show that mesocosm CWs were functioning as treatment systems by actively removing organic matter and $\mathrm{PhACs}$ in the influent. CWs were started up within 10 days and showed a varying removal performance for the PhACs tested: low to moderate removal for carbamazepine (CBZ, 7\% median) and diclofenac (DFC, 46\%), and high removal for caffeine (CAF, 99\%), naproxen (NAP, 89\%), metoprolol (MET, 85\%), propranolol (PRO, 84\%), and ibuprofen (IBP, 79\%).

Secondly, all seven PhACs were detected in substrate and plant tissues, including roots and rhizomes (RR tissues), and stems and leaves (SL tissues). Intermediate hydroxyl-IBP was detected in the aqueous phase, substrate and plant tissues as well. Another intermediate glucopyranosyloxy-hydroxy-IBP was observed in RR tissues. Thirdly, mass balance of PhACs in CWs was estimated, from which we found sorption dominated PRO removal while other six PhACs were mainly removed by biodegradation and/or phytodegradation.

Finally, pre-photocatalysis significantly increased removal of PhACs except for CAF and IBP, and decreased the accumulation of $\mathrm{PhAC}$ in substrate and plant tissues of the following plant compartment. Litter addition in CW significantly enhanced removal of PRO, CBZ, and IBP via biodegradation and/or phytodegradation. Substrate composition did not affect removal of the other four PhACs. We conclude that plants played an essential and positive role in the removal of all the seven $\mathrm{PhACs}$, resulting from direct phytoremediation (uptake and phytodegradation), and indirectly enhancing sorption and biodegradation.

Our study provides knowledge to understand removal mechanisms of PhACs in CWs and to potentially enhance the performance of CWs toward PhAC removal by enhancing the removal processes. Recommendations include the development of pre-photocatalysis, addition of dead plant biomass, and optimization of vegetation.

\title{
Keywords
}

Pharmaceuticals; Constructed wetlands; Removal processes; Pre-photocatalysis; Substrate composition; Effect of plants 


\subsection{Introduction}

The accumulation of pharmaceutically active compounds (PhACs) in the environment and their risks to terrestrial and aquatic ecosystems at environmental concentrations ( $\mathrm{ng} / \mathrm{l}$ to $\mu \mathrm{g} / \mathrm{l}$ ) are confirmed by numerous chemical and toxicological studies [32, 40, 165, 283]. In addition, $\mathrm{PhAC}$ abundance is estimated to increase in the environment due to the increase of population and aging, and affordability of medicines [208]. As a main barrier to prevent $\mathrm{PhACs}$ from entering the environment, conventional wastewater treatment plants (WWTPs) do not remove PhACs sufficiently. A review revealed that 24 out of 50 investigated PhACs were removed less than 50\% in WWTPs [359]. To further eliminate PhACs from wastewater effluent, post-treatment processes urgently need to be established.

A constructed wetland $(\mathrm{CW})$ is a promising post-treatment technique for $\mathrm{PhAC}$ removal [224]. Compared to other techniques, such as activated carbon adsorption, membrane based technologies and advance oxidation techniques, $\mathrm{CW}$ has lower operation and maintenance costs, and can be integrated into the landscape [100]. Removal of PhACs in CWs is complex, with different processes occurring, namely photodegradation, sorption, biodegradation, and phytoremediation [400]. These processes are the natural attenuation processes of PhACs in the environment. Therefore, $\mathrm{CW}$ is an integrated and enhanced version of the natural ecosystem for removal and transport of PhACs. Moreover, PhACs in nature end up mainly in soil/sediment, water, and plants, which are also components of CWs. Investigation of PhAC removal in CWs can give insights into the fate and distribution of PhACs in the environment.

To date, studies of PhAC removal in CWs mostly focus on evaluation of overall $\mathrm{CW}$ removal performance $[8,57,64,223,360]$ and effect of operational and environmental parameters on the performance $[14,16,105,170,311,395]$. In comparison, much less work demonstrates the fate of PhACs in CWs and their distribution in the various matrices of CWs, including water, plants, and substrate (mainly sediment). There are several studies that demonstrate photodegradation, biodegradation, and phytoremediation of PhACs in hydroponic mesocosm CWs [265, 397, 409], instead of in substrate-based CWs. In this way, the role of sediment, and the interaction between sediment and plants for PhAC removal cannot be identified. Therefore, more research needs to be conducted to understand the fate of PhACs in the various removal processes and distribution of PhACs in the various matrices in substratebased CWs. 


\section{Integrated constructed wetland}

To enhance the performance of $\mathrm{CW}$ for PhAC removal, most of the studies engage on optimizing operational parameters, including configuration, vegetation, feeding regime, hydraulic retention time (HRT), substrate material and aeration as described previously. In addition, irradiation and organic matter can effect $\mathrm{PhAC}$ removal as well. Photodegradation of PhACs could be enhanced via solar light driven photocatalysis [156, 276]; sorption and biodegradation of PhACs can be enhanced by addition of organic matter [157, 318]. To date, the enhancement of PhAC removal through photocatalysis and organic matter addition are not yet applied in CW studies. To this end, it is of significance to explore the effect of photocatalysis and $\mathrm{OM}$ addition on $\mathrm{PhAC}$ removal in CWs.

In this study, we investigated the fate and distribution of seven PhACs in substrate-based mesocosm CWs. Additionally, the effect of irradiation via pre-photocatalysis, substrate composition through litter (dead plant biomass) addition, and plants were investigated on PhAC removal. Our study provides knowledge to understand removal mechanisms of PhACs in $\mathrm{CWs}$ and to potentially enhance the performance of $\mathrm{CWs}$ toward $\mathrm{PhAC}$ removal by enhancing the removal processes.

\subsection{Materials and methods}

\subsubsection{Chemicals and reagents}

PhACs including caffeine (CAF), metoprolol (MET), propranolol (PRO), ibuprofen (IBP), naproxen (NAP), diclofenac (DFC), and carbamazepine (CBZ), and internal standards including fenoprofen and dihydrocarbamazepine were purchased from Sigma-Aldrich (USA). Physicochemical properties of the seven PhACs are shown in Table S2.1 of Supplementary Materials. All other chemicals used were of analytical grade (Text S8.1).

\subsubsection{Mesocosm CWs}

To investigate removal of seven PhACs in CWs and the effect of irradiation, substrate composition, and plant on PhAC removal, four CWs were investigated: CW1, CW2 with prephotocatalysis, CW3 with litter addition, and CW4 in the absence of plants (Figure 8.1). CW2 has an extra compartment for pre-photocatalysis with a $10 \mathrm{~cm}$ water depth. Five glass containers $\left(25 \times 25 \times 25 \mathrm{~cm}^{3}\right)$ without gluing are used to avoid PhAC sorption to the containers. All CWs were operated in a horizontal subsurface flow mode. 
Mesocosm CWs were fed with a mixture of two flows; wastewater effluent and a mixture of seven $\mathrm{PhACs}$ solution ( $25 \mathrm{mg} / \mathrm{l}$ in 1\% methanol). PhAC concentration in the influent was 100 $\mu \mathrm{g} / \mathrm{l}$ each. This mixed flow was pumped from a refrigerator $\left({ }^{\circ} \mathrm{C}\right)$ to the $\mathrm{CWs}$ at a flow rate of $3 \mathrm{l} / \mathrm{d}$. The wastewater effluent was collected from Bennekom WWTP (the Netherlands) in a large amount and stored at $4{ }^{\circ} \mathrm{C}$ to maintain $\mathrm{CW}$ operation during experiments. Concentrations of nitrate and sulfate of the wastewater effluent were 0.6 and $40 \mathrm{mg} / \mathrm{l}$, respectively. Nitrite and phosphate were not detected. $\mathrm{pH}$ of the wastewater effluent was 7.7 with conductivity of $7.6 \mu \mathrm{S} / \mathrm{cm}$. To avoid pump clogging, wastewater was filtrated with 7.5 $\mu \mathrm{m}$ polyethylene filters (ProSense, the Netherlands) that were changed every three days.

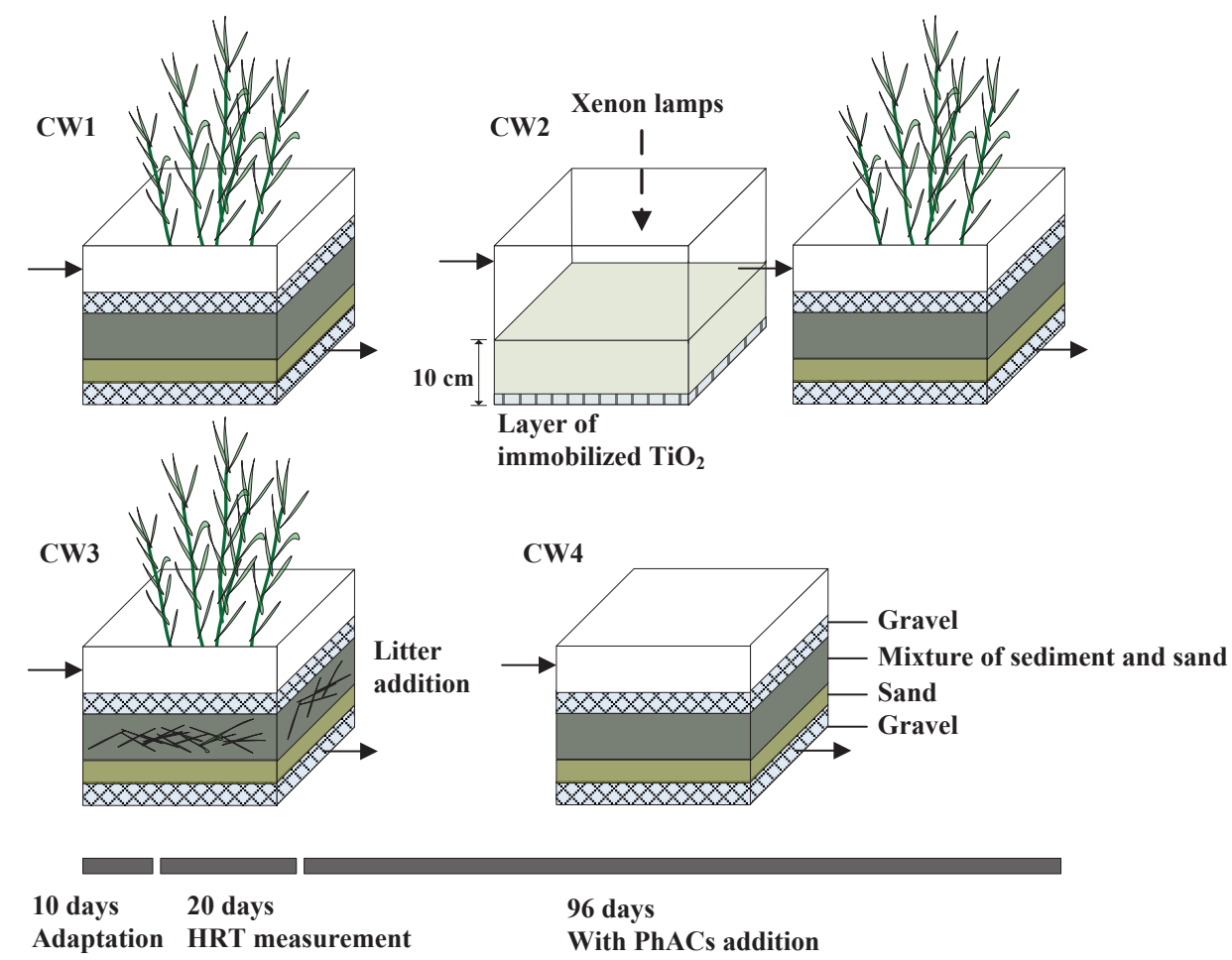

Figure 8.1 Schematic diagram of the four mesocosm constructed wetlands.

The substrate of each $\mathrm{CW}$ consisted of four layers, from bottom to top: $4 \mathrm{~cm}$ gravel, $4 \mathrm{~cm}$ sand, $8 \mathrm{~cm}$ mixture of sediment and sand, and $4 \mathrm{~cm}$ gravel (Figure 8.1). Sediment and sand were homogeneously mixed in a ratio of 1:1 (dry weight) by a mixer and added to CW1, CW2, and CW4. In CW3, litter was added as the additional organic matter to a mixture of sediment and sand in a ratio of 8:25:25 (dry weight) and mixed homogeneously. Gravel (average diameter of $12 \mathrm{~mm}$ ) and sand (average diameter of $0.2 \mathrm{~mm}$ ) were purchased from 


\section{Integrated constructed wetland}

GAMMA (the Netherlands). The sediment and litter were collected from a CW located at a Dutch WWTP as a post-treatment process.

CW1, CW2 and CW3 were vegetated with Phragmites australis, which was purchased from a nursery Jörg Petrowsky (Wasserpflanzengärtnerei, Germany). Soil, the original support material of the plants, was replaced with our experimental substrate. Plants were precultivated in a climate chamber (Heraeus Vötsch, Germany) and fed with wastewater effluent for three months. Well-grown plants were transferred to mesocosm CWs for experiments.

Experiments were performed in the climate chamber to maintain a relative humidity of $40 \%$ (day) and $60 \%$ (night), temperature of $23^{\circ} \mathrm{C}$ (day) and $20^{\circ} \mathrm{C}$ (night) in 12 day/night hours. Fluorescent lamps (58 W, 400-800 nm, 645 lux) were used to support plant growth. Xenon high-intensity discharge lamps (55 W, 300-800 nm, $6280 \mathrm{lux}$ ) were applied to simulate solar light for photocatalysis [156]. Immobilized $\mathrm{TiO}_{2}(165 \mathrm{~g})$ was placed at the bottom of the light compartment of CW2. The immobilization procedure of $\mathrm{TiO}_{2}$ was used as previously described [156]. HRT of our four CWs were measured as approximate 3 days by impulsively injecting sodium fluorescein to the influent. Detailed HRT measurement procedure is shown in Text S8.2. During the whole experiment of 125 days, mesocosm CWs were first started with adaptation by feeding with wastewater effluent only (10 days), followed by HRT measurement (20 days), and finally addition of PhACs (Figure 8.1). The following data related to PhACs are from day 30 when PhACs were added.

\subsubsection{Sampling and analysis}

Concentration of PhACs and potential intermediates of IBP were measured in the aqueous phase, substrate, and plant tissues, respectively. Samples in the aqueous phase were withdrawn every day in the first 10 days of $\mathrm{PhAC}$ addition to monitor stabilization, followed by a sampling frequency of every three days. PhAC concentrations in the aqueous phase were analyzed on an ultra-performance liquid chromatography (UPLC) equipped with diode array detection based on the description by He et al. [156].

Homogenous substrate and plant tissues in four CWs were collected in the beginning and the end of experiments. PhACs were extracted from substrate in quadruplicate according to a method adjusted from a work of Headley and Kadlec [160]. Plant tissues were harvested in two parts: roots and rhizomes (RR tissues), and stems and leaves (SL tissues). Extraction of PhACs from plant tissues was in triplicate as previously described [158]. Details of the 
extraction methods for substrate and plant tissues are illustrated in Text S8.3. PhAC concentration in substrate and plant tissues were measured by a Waters UPLC-Xevo Triple Quadrupole mass spectrometry (MS) according to the protocol demonstrated by [159]. Potential intermediates of IBP in aqueous samples, substrate, and plant tissues were analyzed by a Thermo UPLC-MS/MS as described by He et al. [158]. In addition, real-time inflow rate and effluent volume of four CWs were measured during sampling to calculate mass balance of PhACs.

Quantity and quality of the substrate were determined in four CWs before and after the 125 days of operation. Quantification of substrate was conducted by measuring organic matter gravimetrically after drying at $105{ }^{\circ} \mathrm{C}$ following combustion at $550{ }^{\circ} \mathrm{C}$. Qualification of substrate was determined by characterizing fractions and functional groups of dissolved organic carbon (DOC) extracted from the substrate. DOC fractions comprised of humic acid (HA), fulvic acid (FA), hydrophilic compounds (Hy), and hydrophobic neutrals (HON). Extraction and fractionation of DOC was conducted as described in a previous work study [157]. To characterize functional groups, the extracted DOC was first freeze-dried to solid material by an alpha 2-4 LD+ freeze dryer (Martin Christ GmbH, Germany) and then characterized using a Bruker Tensor 27 Fourier transform infrared spectroscopy (FTIR) equipped with a Platinum ATR accessory (Bruker, Germany).

Basic water qualities were measured as follows. Chemical oxygen demand (COD) was determined by Dr. Lange test kits (Hach Lange GmbH, Germany). Total organic carbon (TOC) was measured by a TOC analyzer (Shimadzu, Japan). Negative ions as described previously were measured by an ion chromatography according to the method demonstrated in a work of De Wilt et al. [375]. pH and conductivity were analysed by a multi-parameter digital meter (Hach HQ40d, USA).

\subsubsection{Statistical analysis}

Significant differences of PhAC removal between CW1 and other CWs were established by the analysis of variance method (ANOVA, single factor) at different significance levels. Comparisons were considered significantly different for $* \mathrm{P}<0.05, * * \mathrm{P}<0.01$ and $* * * \mathrm{P}<$ 0.001 . 


\subsection{Results and Discussion}

\subsubsection{Removal of organic matter in aqueous phase}

Activity and function of mesocosm CWs as treatment systems was observed in the active removal of COD and TOC. COD and TOC in the influents of four CWs were on average 71 and $29 \mathrm{mg} / \mathrm{l}$ respectively (Table S8.1). Considering the low level of PhACs in the influent (100 $\mu \mathrm{g} / \mathrm{l}$ each), COD and TOC detected are mainly contributed from the wastewater effluent. Removal of COD and TOC was in the range of $42-66 \%$ and $53-63 \%$ respectively without obvious difference in our four CWs (Table S8.1). A comparable removal of organic matter was reported in a horizontal subsurface flow $\mathrm{CW}$, where the removal of dissolved organic carbon is $37 \%$ [372]. In our study, the light compartment of CW2 showed negligible removal of COD and TOC (Table S8.1), indicating that organic matter in the wastewater effluent are non-photodegradable. Additionally, no shift of organic matter signature was observed in $\mathrm{CW}$ treatment when comparing TOC/COD ratios of influents and effluents of four CWs (Figure $\mathrm{S} 8.1$ ). The unchanged signature reveals that removal processes in mesocosm CWs favored neither oxidized nor reduced carbon species. Considering the previously found positive correlation between removal of organic matter and removal of PhACs [159], PhAC removal is expected in the mesocosm CWs due to the active removal of COD and TOC.

\subsubsection{Fate and of PhACs in CWs}

\subsubsection{PhAC removal in aqueous phase}

The mesocosm CWs were started up for PhAC removal within 10 days after PhAC addition and showed an overall high removal performance from that time. Taking CW1 as an example, after the start-up stage CW1 showed significant removal of PhACs except for CBZ (Figure 8.2). CBZ was likely sorbed in the first 10 days of PhAC addition and then underwent a breakthrough almost without any removal. In line with our finding, CBZ removal in CWs was explained by sorption onto the substrate in CWs [257]. As one of the most recalcitrant $\mathrm{PhACs}$ in aquatic environment, $\mathrm{CBZ}$ was refractory to biodegradation [9]. The recalcitrant behaviour of CBZ was also found in the other three mesocosm CWs (Figure S8.2). 


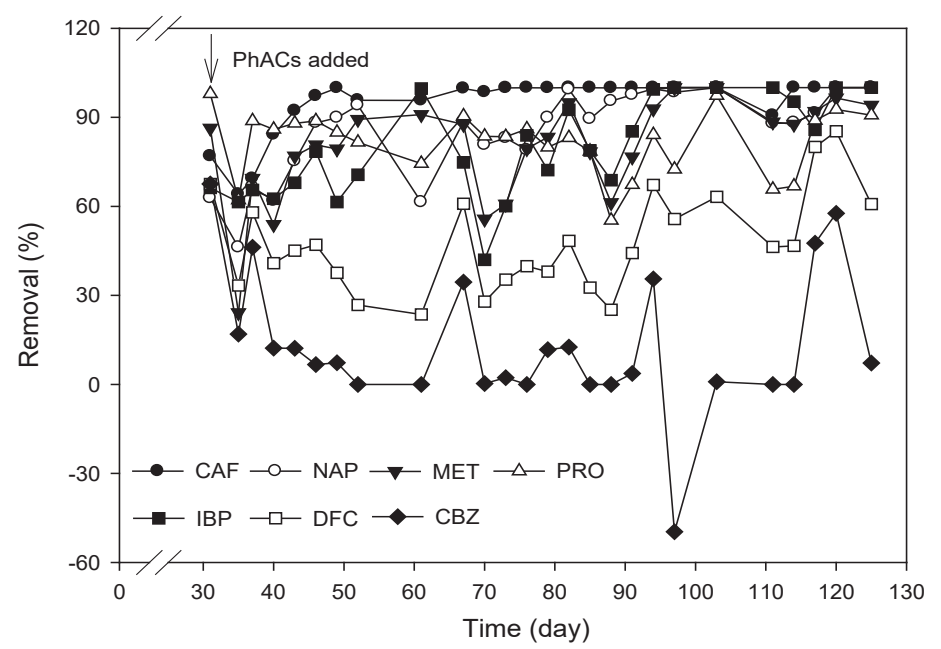

Figure 8.2 Removal efficiencies of seven PhACs in CW1. PhACs were added to the CW at day 30 of operation.

Median removal of CAF, NAP, MET, PRO, and IBP in CW1 was in the range of 79-100\%, as indicated in Figure 8.3a. Especially CAF was continuously and almost completely removed after the initial period of 10 days. Similarly, a previous study observed a high removal of CAF (82-99\%) in CWs at various configurations during the summer season in northwest Spain [169]. The efficient removal of CAF was also observed in CW2, CW3, and CW4 (Figure S8.2). Limited removal of DFC $(<45 \%)$ was found in horizontal subsurface flow CWs $[258,262]$, which is similar with our observation (46\% median). After prolonged operation, CW1 showed an increased removal of DFC after day 94 (Figure 8.3a), which indicates the potential of $\mathrm{CW}$ to adapt for DFC removal. 


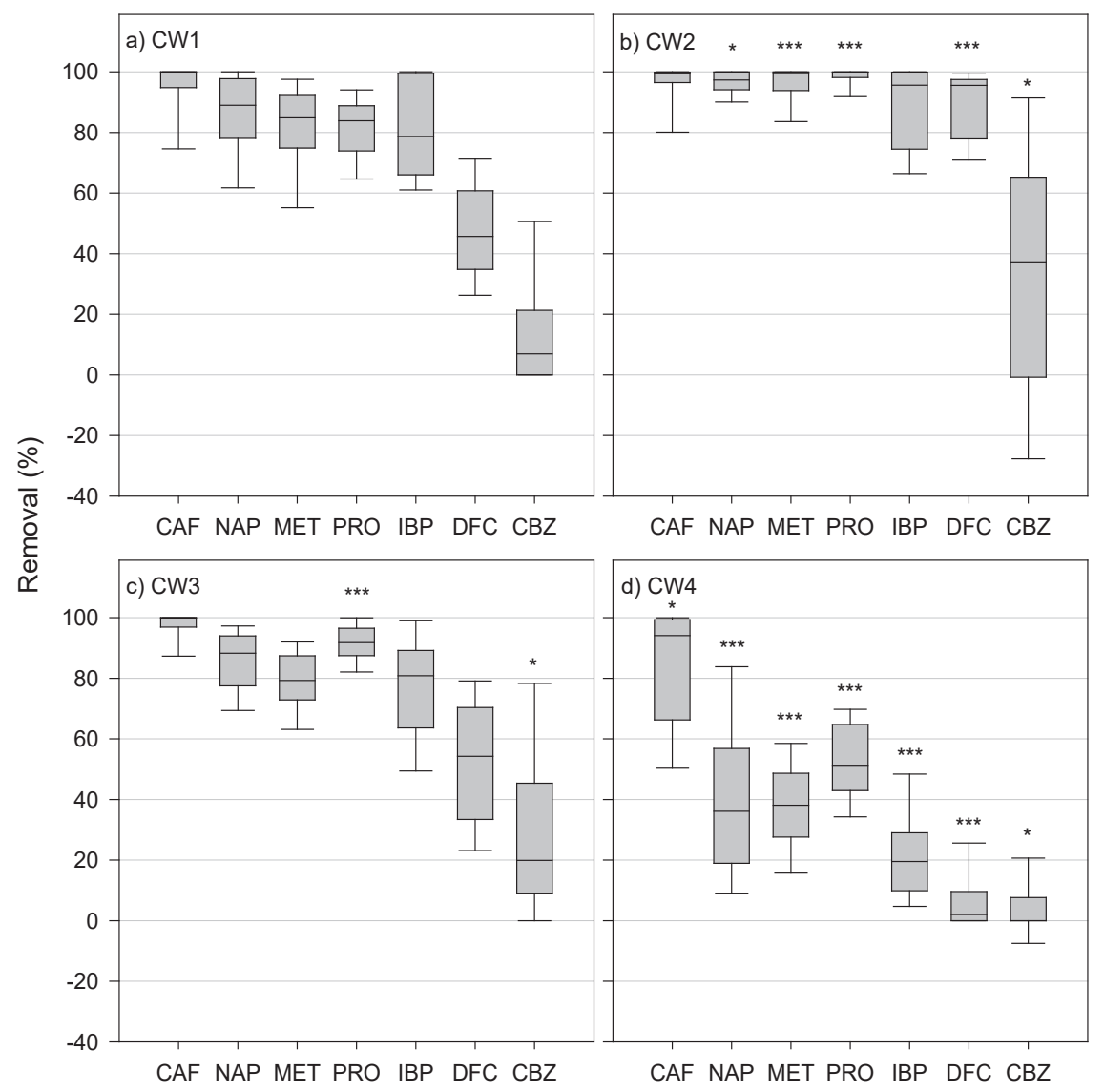

Figure 8.3 Removal efficiencies of seven PhACs in four mesocosm CWs a) CW1, b) CW2, c) CW3, d) CW4. The box plot shows the values in maximum, third quartile, median, first quartile, and minimum. $\mathrm{n}=26,24,25$, and 29 respectively for four CWs. The indicated significance was comparison between $\mathrm{CW} 1$ and others.

\subsubsection{PhAC abundance in substrate}

Due to litter addition, organic matter of CW 3 substrate was of a different quantity and quality as compared to the other three. CW3 contained $32.3 \mathrm{~g} / \mathrm{kg}$ (dry substrate matter) organic matte while the content was $29.8 \mathrm{~g} / \mathrm{kg}$ in the other three CWs. Additionally, organic matter was characterized by performing DOC extraction on the substrate. Differences in DOC composition can be associated with the maturity and microbial activity of the substrate, and thus can be used for substrate qualification [336]. DOC concentration was 40.9 and 35.7 $\mathrm{mg} / \mathrm{kg}$ (dry substrate matter) respectively in CW3 and other three CWs (Figure 8.4). The 
initial DOC in four CWs consists approximate half of hydrophobic acids (HA and FA) and half of hydrophilic fractions and hydrophobic neutrals (Hy and HON; Figure 8.4). Previous work demonstrated that hydrophobic acids contribute for $26 \%$ and $48 \%$ in DOC of wastewater effluent and plant tissues respectively, and factions of Hy and HON contributes $48 \%$ and $36 \%$ respectively [372]. Our fractionation results imply that the predominance of stable organic matter fractions in $\mathrm{CW}$ substrate originates from wastewater and wetland plant litter, as reported previously Nguyen [280]. Compared to the other three CWs, the percentage of HA fraction was higher in CW3 (Figure 8.4). DOC in CW1, CW2, and CW4 contains three main bands corresponding to different functional groups (Figure S8.3): 1) $3390 \mathrm{~cm}^{-1}$ (O-H stretching of inter- and intra- molecular hydrogen bonds); 2) $1639 \mathrm{~cm}^{-1}$ (aromatic $\mathrm{C}=\mathrm{C}$ skeletal vibrations, asymmetric stretching of $\mathrm{C}=\mathrm{O}$, symmetric stretching of $\mathrm{COO}-$ ); 3) 1359 $\mathrm{cm}^{-1}$ (C-O asymmetric stretching of $\mathrm{COOH}$ groups). In comparison, DOC in CW3 contains one more band at $1105 \mathrm{~cm}^{-1}$ (C-O stretching of alcohols and carbohydrate).

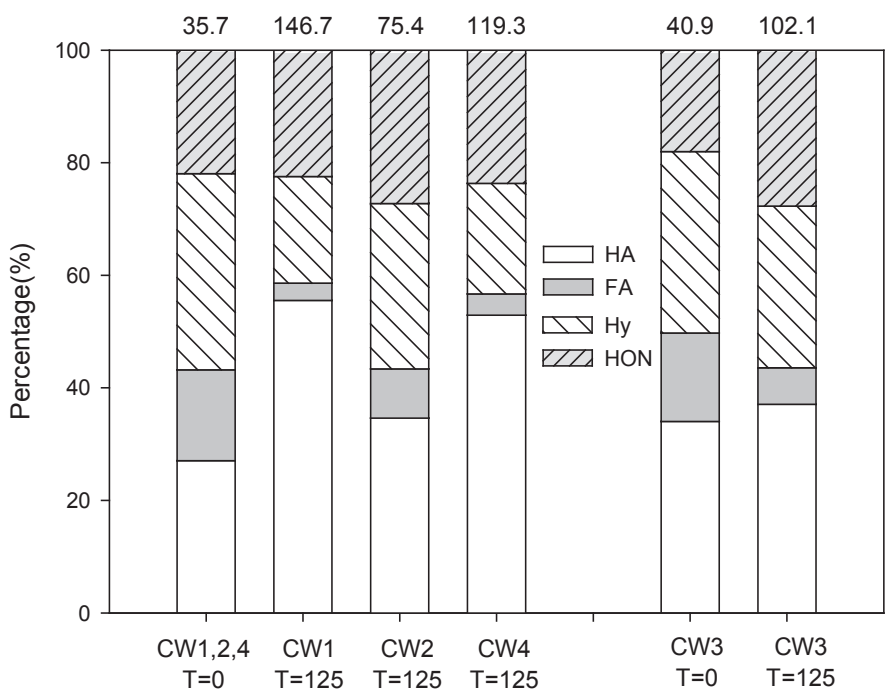

Figure 8.4 DOC fractionation in four mesocosm $\mathrm{CWs}$ at day 0 and $125(\mathrm{HA}=$ humic acid, $\mathrm{FA}=$ fulvic acid, Hy = hydrophilic compounds, $\mathrm{HON}=$ hydrophobic neutrals). Numbers on the top of the figure are DOC concentrations in unit of $\mathrm{mg}$ carbon $/ \mathrm{kg}$ dry substrate matter.

Abundance of PhACs in the substrate of four CWs at day 0 and 125 is depicted in Table 8.1. Notably, all seven PhACs tested were detected in the initial substrate at day 0 except for NAP. This finding indicates that PhACs in the WWTP and field CW where we collected sediment were incompletely removed and accumulated in the $\mathrm{CW}$ sediment. At day $125 \mathrm{PhAC}$ 


\section{Integrated constructed wetland}

concentrations in the substrate of CW2 were less than the other three CWs. Apparently, part of PhACs was already removed in the light compartment of CW2 resulting in less PhAC load entering CW2 (described in section 3.3.1). While sorption capacity of PhACs can be related to their hydrophobicity and molecular charge $[48,375]$, in our study it is not feasible to interpret $\mathrm{PhAC}$ concentrations in the substrate according to their physicochemical properties, as other removal processes were also involved. Last, the absolute amount of PhACs sorbed to the substrate was different in four CWs. However, substrates in four CWs showed similar patterns of sorption capacity among different PhACs, indicated by the linear relationship of the distribution of seven PhACs between CWs $\left(\mathrm{R}^{2}>0.97\right.$, Figure $\left.\mathrm{S} 8.4\right)$. It seems that sorption of $\mathrm{PhACs}$ were in a dynamic equilibrium in $\mathrm{CWs}$ with various removal processes involved.

\subsubsection{PhAC abundance in plants}

All seven tested PhACs were found to accumulate in both RR and SL tissues (Table 8.1). To date, there are studies in which plant uptake of CAF, NAP, IBP, DFC, and CBZ in wetland plant species was detected [104, 158, 226, 396, 398, 399]; our data showing $\beta$-blocker uptake (i.e. MET and PRO) has not been reported previously. To further describe plant uptake, the terms bioaccumulation factor indicating PhAC transfer from the solution to RR tissues and translocation factor indicating transfer from $\mathrm{RR}$ to $\mathrm{SL}$ tissues are of importance to characterize the uptake capacity. In most of these previous studies, PhACs were spiked to the plants only once in hydroponic setups, and thus plant uptake factors can be calculated. However, in our continuously operated setups with occurrence of various removal processes, it is not feasible to estimate the overall plant uptake factors and to compare with other studies.

Additionally, with the occurrence of various removal processes, comparison of abundance among PhACs in plant tissues is not attainable in our CWs. For example, hydrophilic and positively charged CAF can be readily transported together with growing medium to plant tissues with negatively charged cell walls [274, 400]. In our study only low abundance of CAF was found in RR and SL tissues compared to other PhACs (Table 8.1), which may result from either prior removal in the substrate via sorption and biodegradation in the aqueous phase before plant uptake or removal via phytodegradation. 
Table 8.1 Abundance of PhACs in substrate and plant tissues of CWs. Data are mean peak area \pm standard deviations ( $\mathrm{n}=4$ for substrate, $\mathrm{n}=3$ for plant tissues). The units are in $\mathrm{ng} / \mathrm{g}$ dry substrate matter and $\mathrm{ng} / \mathrm{g}$ tissue in fresh weight.

\begin{tabular}{|c|c|c|c|c|c|c|c|c|}
\hline Matrix & CWs & CAF & NAP & MET & PRO & IBP & DFC & $\mathrm{CBZ}$ \\
\hline \multirow{6}{*}{ Substrate } & CW1,2,4 (T=0) & $\begin{array}{l}7.8 \\
(1)\end{array}$ & 0 & $\begin{array}{l}149.9 \\
(50.5)\end{array}$ & $\begin{array}{c}580 \\
(83.9)\end{array}$ & $\begin{array}{c}23.6 \\
(15.9)\end{array}$ & $\begin{array}{c}20.3 \\
(12.6)\end{array}$ & $\begin{array}{c}21.2 \\
(13.6)\end{array}$ \\
\hline & CW3 $(\mathrm{T}=0)$ & $\begin{array}{c}6.2 \\
(0.5)\end{array}$ & 0 & $\begin{array}{l}141.1 \\
(67.3)\end{array}$ & $\begin{array}{c}776.7 \\
(193.5)\end{array}$ & $\begin{array}{l}27.9 \\
(5.6)\end{array}$ & $\begin{array}{l}23.8 \\
(4.8)\end{array}$ & $\begin{array}{l}25.2 \\
(6.1)\end{array}$ \\
\hline & $\mathrm{CW} 1(\mathrm{~T}=125 \mathrm{~d})$ & $\begin{array}{c}13.4 \\
(4)\end{array}$ & $\begin{array}{c}9.3 \\
(1.4)\end{array}$ & $\begin{array}{c}442.9 \\
(157.8)\end{array}$ & $\begin{array}{l}5489.7 \\
(234.1)\end{array}$ & $\begin{array}{l}1280.6 \\
(106.3)\end{array}$ & $\begin{array}{c}1188.3 \\
(26.1)\end{array}$ & $\begin{array}{l}302.9 \\
(12.2)\end{array}$ \\
\hline & $\mathrm{CW} 2(\mathrm{~T}=125 \mathrm{~d})$ & $\begin{array}{l}10.3 \\
(3.2)\end{array}$ & $\begin{array}{c}2 \\
(0.5)\end{array}$ & $\begin{array}{c}169.7 \\
(80)\end{array}$ & $\begin{array}{l}2550.6 \\
(121.7)\end{array}$ & $\begin{array}{c}922.6 \\
(129.9)\end{array}$ & $\begin{array}{c}820.4 \\
(157.6)\end{array}$ & $\begin{array}{c}245.9 \\
(15)\end{array}$ \\
\hline & $\mathrm{CW} 3(\mathrm{~T}=125 \mathrm{~d})$ & $\begin{array}{c}8.2 \\
(1.3)\end{array}$ & $\begin{array}{c}3.9 \\
(1.1)\end{array}$ & $\begin{array}{l}516.4 \\
(99.4)\end{array}$ & $\begin{array}{l}4849.4 \\
(132.6)\end{array}$ & $\begin{array}{c}931.4 \\
(100.3)\end{array}$ & $\begin{array}{l}896.7 \\
(95.8)\end{array}$ & $\begin{array}{c}251.4 \\
\text { (11) }\end{array}$ \\
\hline & CW4 $(\mathrm{T}=125 \mathrm{~d})$ & $\begin{array}{l}10.2 \\
(2.1)\end{array}$ & $\begin{array}{c}5.7 \\
(5.1)\end{array}$ & $\begin{array}{c}541.2 \\
(326.7)\end{array}$ & $\begin{array}{l}5131.1 \\
(103.9)\end{array}$ & $\begin{array}{l}938.8 \\
(77.5)\end{array}$ & $\begin{array}{l}900.5 \\
(70.7)\end{array}$ & $\begin{array}{c}254 \\
(10.1)\end{array}$ \\
\hline \multirow{6}{*}{$\begin{array}{l}\text { Plant } \\
\text { tissues }\end{array}$} & CW1-RR & $\begin{array}{c}0.7 \\
(0.2)\end{array}$ & $\begin{array}{l}23.7 \\
(2.3)\end{array}$ & $\begin{array}{l}33.7 \\
(8.7)\end{array}$ & $\begin{array}{c}515.4 \\
(307.3)\end{array}$ & $\begin{array}{l}402.7 \\
(62.1)\end{array}$ & $\begin{array}{l}398.1 \\
(64.3)\end{array}$ & $\begin{array}{l}291.1 \\
(17.3)\end{array}$ \\
\hline & CW2-RR & $\begin{array}{c}0.4 \\
(0.1)\end{array}$ & $\begin{array}{c}8.5 \\
(4.2)\end{array}$ & $\begin{array}{c}5.4 \\
(2.8)\end{array}$ & $\begin{array}{c}35 \\
(17.1)\end{array}$ & $\begin{array}{l}210.6 \\
(59.3)\end{array}$ & $\begin{array}{l}199.9 \\
(74.2)\end{array}$ & $\begin{array}{l}207.4 \\
(42.9)\end{array}$ \\
\hline & CW3-RR & $\begin{array}{c}0.4 \\
(0.1)\end{array}$ & $\begin{array}{l}34.7 \\
(3.2)\end{array}$ & $\begin{array}{c}48.5 \\
(11.7)\end{array}$ & $\begin{array}{l}273.6 \\
(64.6)\end{array}$ & $\begin{array}{c}516.9 \\
(351.8)\end{array}$ & $\begin{array}{l}300.9 \\
(26.5)\end{array}$ & $\begin{array}{c}257.1 \\
(9.6)\end{array}$ \\
\hline & CW1-SL & $\begin{array}{l}6.7 \\
(2)\end{array}$ & $\begin{array}{l}12.4 \\
(6.1)\end{array}$ & $\begin{array}{l}20.7 \\
(1.5)\end{array}$ & $\begin{array}{l}1664.2 \\
(279.2)\end{array}$ & $\begin{array}{c}1093.6 \\
(74)\end{array}$ & $\begin{array}{c}1080.3 \\
(77.8)\end{array}$ & $\begin{array}{c}434 \\
(13.9)\end{array}$ \\
\hline & CW2-SL & $\begin{array}{c}2.6 \\
(0.8)\end{array}$ & $\begin{array}{c}8.1 \\
(1.7)\end{array}$ & $\begin{array}{c}8.6 \\
(0.5)\end{array}$ & $\begin{array}{c}579 \\
(315.5)\end{array}$ & $\begin{array}{l}863.5 \\
(36.6)\end{array}$ & $\begin{array}{l}854.4 \\
(37.2)\end{array}$ & $\begin{array}{c}394.7 \\
(8.7)\end{array}$ \\
\hline & CW3-SL & $\begin{array}{l}13.1 \\
(1.7)\end{array}$ & $\begin{array}{l}12.6 \\
(2.3)\end{array}$ & $\begin{array}{l}29.2 \\
(4.1)\end{array}$ & $\begin{array}{l}2434.5 \\
(313.7)\end{array}$ & $\begin{array}{c}1000.5 \\
(84.8)\end{array}$ & $\begin{array}{l}992.6 \\
(83.4)\end{array}$ & $\begin{array}{l}414.6 \\
(14.3)\end{array}$ \\
\hline
\end{tabular}

Notes: $\mathrm{RR}=$ roots and rhizomes, $\mathrm{SL}=$ stems and leaves. No PhACs were detected in plant tissues at day 0 . Values in brackets are standard deviations.

\subsubsection{Mass balance of PhACs in mesocosm CWs}

Mass balances for the seven tested PhACs were calculated in four mesocosm CWs (Figure 8.5). Contribution of other removal processes was estimated by subtracting $\mathrm{PhAC}$ mass in substrate, plant tissues and $\mathrm{CW}$ effluent from the mass in $\mathrm{CW}$ influent. Photodegradation of PhACs in CW2 was calculated based on the mass difference in the influent and effluent of the light compartment. In other three CWs, we assume that photodegradation was negligible at subsurface flow conditions. Therefore, the other removal processes demonstrated in Figure 8.5 mainly include biodegradation and phytodegradation. 


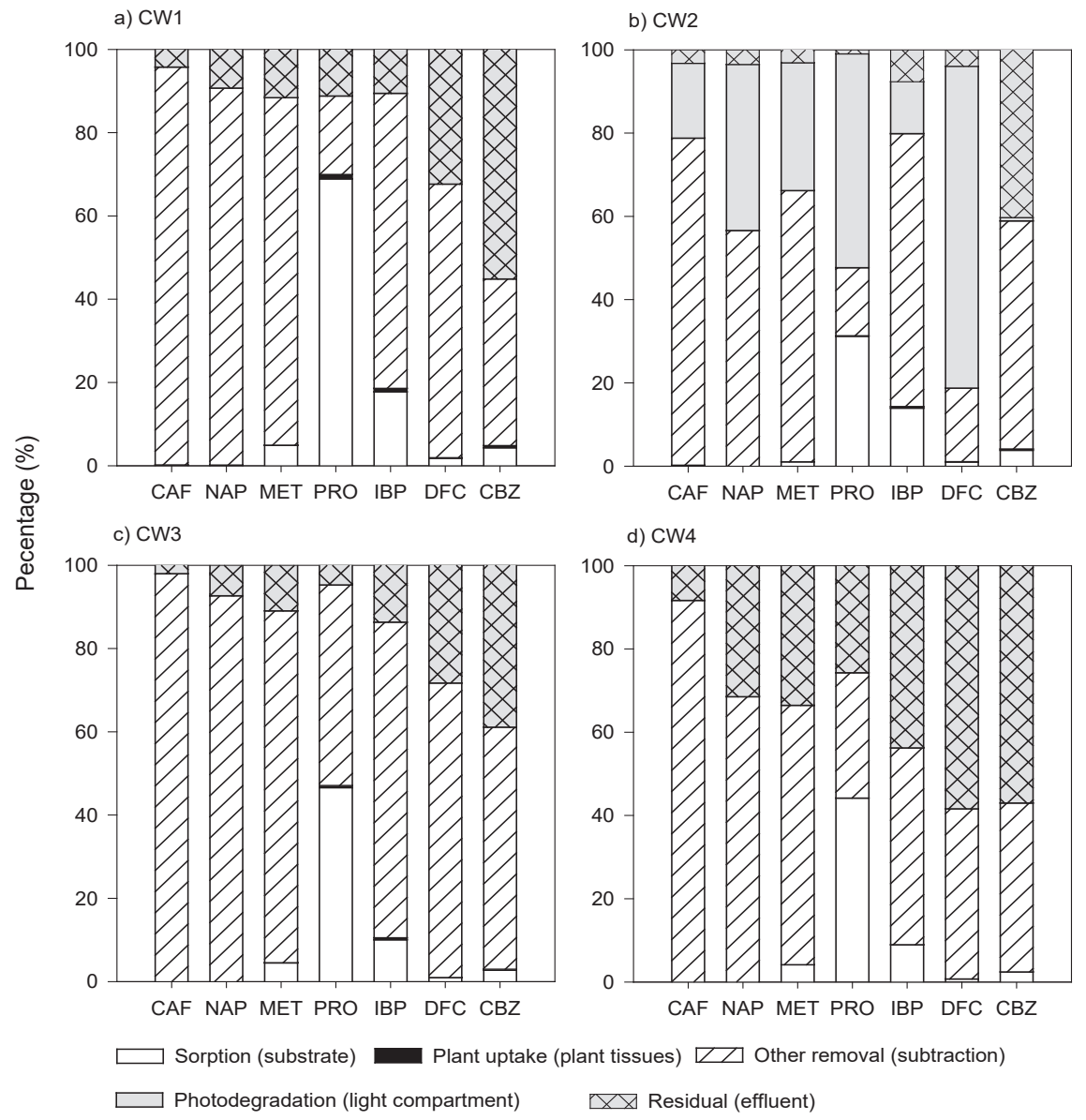

Figure 8.5 Mass balance of seven PhACs in four mesocosm CWs a) CW1, b) CW2, c) CW3, d) CW4.

Various removal processes played a role in removal of PhACs. Sorption dominated PRO removal in CW1, CW3, CW4, and the plant compartment of CW2 (Figure 8.5). With a pH range of 7.4-8.3 in CWs, PRO was hydrophobic ( $\log \mathrm{D}_{\mathrm{ow}}$, Table S2.1) and positively charged. Most of the sediment matrices are overall negatively charged [251,386], which leads to easy sorption of PRO in addition to its hydrophobic property. In comparison, other six PhACs were mainly removed by biodegradation and phytodegradation in the plant compartment of CW2 and other CWs, especially for CAF and NAP. In our previous studies, CAF and NAP showed high removal potential via both aerobic and anaerobic biodegradation in microbial cultures originating from CW sediment, compared to the other five PhACs tested [157, 375]. Comparison of mass balances among CWs will be described in section 3.3. 


\subsubsection{IBP intermediates in mesocosm CWs}

Intermediates of IBP were measured in the aqueous phase, substrate, and plant tissues of CWs. Hydroxy-IBP was detected in the aqueous phase and substrate in all four CWs. In the aqueous phase in the light compartment of CW2, occurrence of hydroxy-IBP was also observed resulting from photodegradation. Dihydroxy-IBP and carboxy-IBP were found in biodegradation studies of IBP [124, 226], but were not observed in our studies reported here. In both aqueous (Table S8.2) and substrate samples (Figure 8.6), concentrations of hydroxylIBP were higher in CW4 compared to the other CWs. Considering the low removal of IBP in CW4 (20\% median, Figure 8.3d), the higher abundance of hydroxyl-IBP in CW4 may relate to the incomplete removal of IBP. In the initial substrate, hydroxyl-IBP was already present, suggesting the occurrence and degradation of IBP either in the WWTP or in the CW where the sediment was collected.

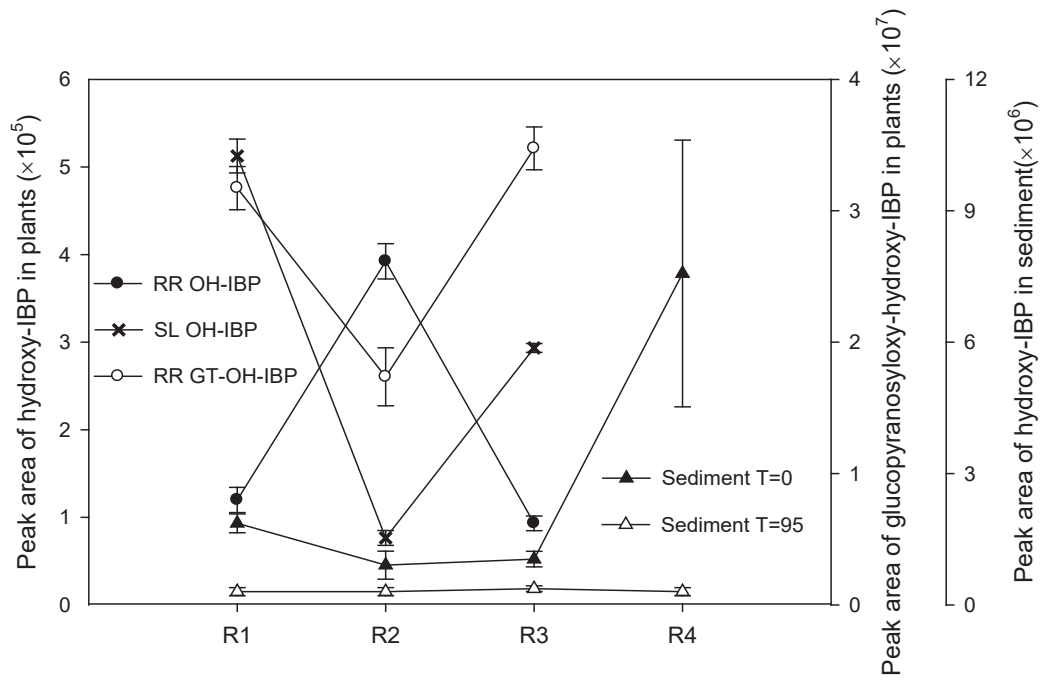

Figure 8.6 Ibuprofen intermediates in substrate and plant tissues $(\mathrm{RR}=$ roots and rhizomes, $\mathrm{SL}=$ stems and leaves). OH-IBP = hydroxyl-IBP; GT-OH-IBP = glucopyranosyloxy-hydroxy-IBP. Chromatogram peak area is the relative concentration indicator for intermediates in substrate and plant tissues (mAU/g substrate (wet weight) and mAU/g tissue (fresh weight)). Data are mean peak area \pm standard deviation ( $\mathrm{n}=4$ for substrate, $\mathrm{n}=3$ for plant tissues). Dots were linked with lines to make them more visible in the figure.

IBP intermediates were also found in plant tissues: hydroxyl-IBP and glucopyranosyloxyhydroxy-IBP (GT-hydroxy-IBP) in RR tissues, and hydroxyl-IBP in SL tissues (Figure 8.6). In contrast with the outcome of a previous study where IBP was spiked once to P. australis 


\section{Integrated constructed wetland}

and monitored for 21 days [158], dihydroxy-IBP and carboxy-IBP were not detected in the plant tissues. We hypothesize that those intermediates were not accumulated but further converted to other intermediates in the tissues, and thus not detectable. Notably, the abundance of IBP was higher in SL tissues of CW2 whereas the abundance of hydroxy-IBP was lower compared to $\mathrm{CW} 1$ and CW3 (Table 8.1, Figure 8.6). The complementary occurrence of IBP and hydroxy-IBP indicates that hydroxy-IBP was phytodegraded from IBP in RR tissues instead of transported from the aqueous phase or substrate. Accordingly, the complementary occurrence of hydroxy-IBP and GT-hydroxy-IBP were found in RR tissues, which implies the conversion from hydroxy-IBP to GT-hydroxy-IBP in the plant tissues.

\subsubsection{Comparison between mesocosm CWs}

\subsubsection{Effect of pre-photocatalysis on PhAC removal}

Pre-photocatalysis significantly increased removal of PhACs except for CAF and IBP, as presented in Figure 8.3b. Pre-photocatalysis only degraded 32\% CAF and $19 \%$ IBP in median (Figure 8.7). In line with previous studies, photodegradation plays a minor role of CAF and IBP removal $[362,400]$. NAP, MET and PRO were readily removed in CW1 but can be further removed due to pre-photocatalysis in CW2 (Figure S8.2). Sensitivity of PRO to solar light driven photodegradation has been well studied [10, 156, 209], compared to the less reported photodegradation of NAP and MET under solar light irradiation.

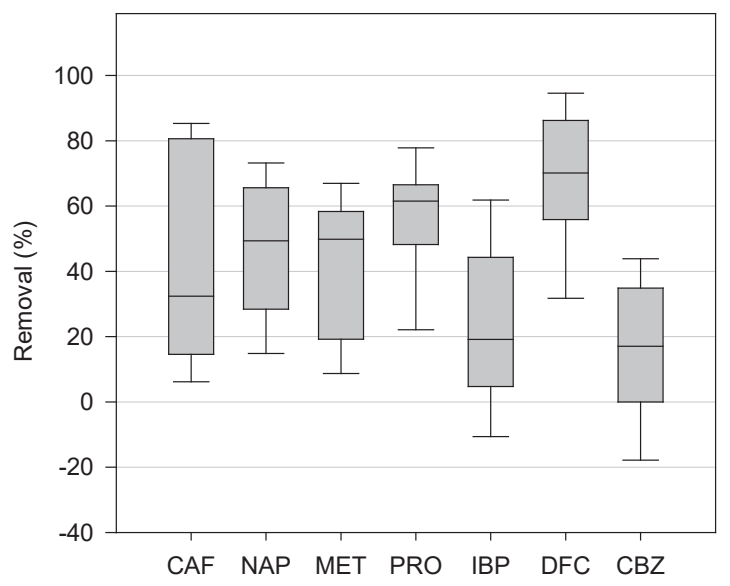

Figure 8.7 Removal efficiencies of seven PhACs in the light compartment of CW2. The box plot shows the values in maximum, third quartile, median, first quartile, and minimum $(n=22)$. 
Removal efficiencies of the two recalcitrant PhACs in CW1, DFC and CBZ, were significantly enhanced in CW2 (Figure 8.3b). Especially, removal of DFC increased from 46\% in CW1 to $96 \%$ in CW2 (median). Enhancement of DFC removal via photodegradation has been frequently reported $[10,311,362,400]$. As indicated in our study, pre-photocatalysis can be a strategy to enhance removal for the more recalcitrant CBZ. In addition to degrading parent CBZ, photodegradation has the capacity to further degrade CBZ intermediates as well, such as 2-hydroxycarbamazepine and 3-hydroxycarbamazepine, as observed by Rühmland et al. [311].

By enhancing PhAC photodegradation, the pre-photocatalysis reduced $\mathrm{PhAC}$ accumulation in substrate and plant tissues of the following plant compartment, compared to the related $\mathrm{PhAC}$ accumulation in CW1 (Table 8.1, Figure 8.5). Therefore, for the long-term operation of CWs, establishment of pre-photocatalysis can slow down the potential breakthrough of PhAC sorption and reduce the risk of excessive PhAC accumulation in plant tissues, which may introduce toxicity to the plants [58].

\subsubsection{Effect of substrate composition on PhAC removal}

Litter addition in CW3 significantly enhanced removal of PRO and CBZ, but had no effect on the other PhACs, as indicated in Figure 8.3c. Removal of PRO was notably higher in CW3 compared to CW1 due to the litter addition (Figure 8.2, Figure S8.2). CBZ showed a slower breakthrough in CW3 compared to CW1 (Figure S8.2), possibly due to the addition of litter for retention in the CW. Schaffer et al. [318] demonstrated increased removal of 12 out of 28 organic pollutants, including NAP, IBP, MET, and DFC, by introducing a compost layer to soil aquifer columns. The authors ascribed the increased removal to sorption and biodegradation. In our study, the enhancement is mainly due to biodegradation and/or phytodegradation as illustrated in the mass balance results (Figure 8.5). From Figure 8.5 the enhancement of biodegradation and/or phytodegradation was also found for IBP by comparing CW3 and CW1.

In comparison, no significant effect of substrate composition was observed for the other four PhACs, which may be explained by the relatively low quantity of litter we added. As demonstrated in our previous work [157], in which the same source of litter was applied as in the study reported here, addition of DOC extracted from litter enhanced biodegradation of MET, NAP, and IBP. Moreover, differences in substrate quality between CW3 and the other three CWs decreased after 125 days operation. The initial quality difference was 


\section{Integrated constructed wetland}

characterized by higher DOC concentration, higher HA fractions, and additional functional groups in the substrate of CW3. However, after 125 days operation, DOC concentration and HA fractions were no longer higher in the substrate of CW3. Concentration of DOC significantly increased in CW1, CW2, and CW4 as well as HA fractions (Figure 8.4). Although CW4 had no plants, algae pervaded on the substrate of CW4 which may have contributed to the observed humification. In addition, the band $1105 \mathrm{~cm}^{-1}$ was observed in all four CWs after 125 days operation (Figure S8.3). Thus, the higher quantity of organic matter in CW3 might be the key for its higher removal of PRO and CBZ, compared to other three CWs.

\subsubsection{Effect of plants on PhAC removal}

Removal of all the PhACs tested was significantly lower in CW4 with the absence of plants (Figure 8.3d). In line with previous studies, plants play an essential and positive role in $\mathrm{PhAC}$ removal, as confirmed for CAF, NAP, IBP, and DFC [224, 394]. There are several reasons for the observed enhancement. First, plants can directly take up PhACs (Table 8.1) and further phytodegrade PhACs (e.g. IBP, Figure 8.6). Phytodegradation of CBZ was also found in a wetland plant species, Typha spp., by detecting an intermediate 10,11-dihydro-10,11epoxycarbamazepine [104]. Second, rhizodegradation of PhACs can be promoted with plants $[158,226]$. Plant roots can release oxygen and diverse exudates such as ions, inorganic and organic acids, proteins, and enzymes. The released oxygen can favor aerobic biodegradation in the rhizosphere. The organic acids and proteins can act as additional carbon source, and enzyme can catalyze the reaction [158]. It is reported that agricultural crops can release $10-40 \%$ of the net photosynthetic production to the rhizosphere [163]. In addition, plant roots can clog up pores in the substrate (Figure S8.5), which may result in transport of more oxygen and enhancement of aerobic biodegradation of PhACs. Last, the root exudates can strongly impact soil properties and thus influence sorption of neutral and ionizable organic contaminants [274]. From the mass balance outcomes (Figure 8.5), the overall sorption of PRO, IBP, DFC, and CBZ was higher in CW1 compared to CW4, thus confirming the influence of plants in the sorption.

\subsubsection{Implication for CW application}

Photodegradation, sorption, biodegradation, and phytoremediation all play an important role in CWs for PhAC removal. Based on our study, those removal processes can be strengthened by optimizing conditions of irradiation, substrate composition, and plants. First, pre- 
photocatalysis can reinforce solar light irradiation to photodegrade PhACs in the light compartment, and release the burden of $\mathrm{PhAC}$ accumulation in substrate and plant tissues in the following plant compartment. The immobilized $\mathrm{TiO}_{2}$ applied in the light compartment is a robust catalyst. During the 125 days of operation, algae were prevalently growing in the light compartment that may attenuate the irradiation, but no negative effect was observed in PhAC removal. Thus, an open compartment with the robust $\mathrm{TiO}_{2}$ catalyst can be established prior to the plant compartment of a CW for a higher PhAC removal.

Second, removal of PhACs can be enhanced by litter addition, preferably in appropriate amounts. Excessive of organic matter in CWs may lead to a series of negative effects on pollutant removal, such as decrease of hydraulic conductivity, hydraulic malfunction, block of oxygen transportation, and reduction of biodegradation [272]. Although in this study only removal of PRO and CBZ was significantly increased when litter was added, there was no negative effect of litter addition on PhAC removal. Additionally, organic matter decreased after 125 days of operation $(23.2,23.9,25.8$, and $26.5 \mathrm{~g} / \mathrm{kg}$ dry substrate matter respectively for four CWs), which also needs to be replenished. Therefore, dead plant biomass (partially) can be applied in CWs to enhance PhAC removal.

Last, considering the essential role of plants in CWs for PhAC removal, optimization of vegetation such as plant species and density might be advantageous. For example, $P$. australis was found to be the best candidate for removing two pesticides, outperforming other commonly applied wetland plant species, including Typha latifolia, Iris pseudacorus and Juncus effuses [243]. A CW with a densely planted Typha latifolia (40 plants $/ \mathrm{m}^{2}$ ) showed a faster removal of chloroform (a disinfection by-product), compared to a relatively sparsely planted CW (22 plants $/ \mathrm{m}^{2}$ ) [69]. Therefore, optimization of vegetation could be another application direction for enhancing $\mathrm{PhAC}$ removal in CWs.

\subsection{Conclusions}

Fate and distribution of seven PhACs in substrate-based mesocosm CWs were investigated. Main conclusions were as follows:

1) CWs showed a short start-up, and for most compounds a high and stable removal. Photodegradation, sorption, biodegradation, and phytoremediation all play an important role in $\mathrm{PhAC}$ removal; 
2) In CWs, all PhACs as well as the IBP intermediates were detected in substrate and in plant tissues;

3) To achieve favorable conditions in CWs for PhAC removal, irradiation, substrate composition, and plants can be further influenced towards higher total removal efficiencies by applying pre-photocatalysis, addition of dead plant biomass, and tailored composition of vegetation.

\section{Acknowledgement}

Authors thank Vinnie de Wilde, Bert Willemsen, and Ilse Gerrits for their technical support for operating reactors. The support provided by China Scholarship Council (CSC) for the research of Yujie He at Wageningen University is kindly acknowledged. 


\section{Supplementary materials}

Text S8.1 Chemicals and reagents

Stock preparation. Methanol of ultra-performance liquid chromatography (UPLC) gradient (Biosolve B.V., the Netherlands) was used to prepare stock solution of pharmaceutically active compounds (PhACs).

Catalyst immobilization. Titanium(IV) butoxide (reagent grade 97\%, Sigma-aldrich ,USA), $\mathrm{HNO}_{3}$ (Merck, Germany), acetone (Biosolve B.V., the Netherlands), ethanol ( $\geq 99 \%$, Merck, Germany) and glacial acetic acid (99-100\%, Merck, Germany) were used to synthesize $\mathrm{TiO}_{2}$.

HRT monitoring. Sodium fluorescein (SF) (Merck, Germany) was used as the tracer to measure hydraulic residence time (HRT) of our mesocosm constructed wetlands (CWs).

PhAC extraction. Acetone, acetic acid, and ethyl acetate (Merck, Germany) were used to extract PhACs from substrate. $\mathrm{HCl}$ (Boom BV, The Netherlands) and acetonitrile (Merck, Germany) were used to extract PhACs from plant. $\mathrm{NaN}_{3}$ (VWR International B.V., The Netherlands) was used to inhibit the microbial activity during the validation of PhACs extraction methods.

Chemical analysis. Acetonitrile, water, methanol (Biosolve B.V., the Netherlands), ammonium formate (Sigma, USA), formic acid (Biosolve B.V., the Netherlands) of HPLC grade were used for detecting PhAC concentration and ibuprofen intermediates in liquid aqueous, substrate, and plants.

Text S8.2 Determination of HRT

HRT is a basic parameter for CWs. Sampling strategy is suggested to be determined based on the actual HRT. The HRT test was guided by the method of Headley and Kadlec [160]. Sodium fluorescein (SF) was tracer to measure HRT of CWs. The concentration of SF was measured by Multimode microplate readers (TECAN Infinite 200 PRO, Austria). The HRT test includes two parts: pre-test of the tracer dosage and HRT determination in CWs.

Pre-test of the tracer dosage. To determine the dosage of SF in each $\mathrm{CW}$, three experiments were carried out: 1) The existing SF concentration in the wastewater effluent and substrate; 2) 


\section{Integrated constructed wetland}

The maximum dosage of SF to each CW; 3) The potential loss of SF in CWs. The detailed of those experiments can be seen as follows:

1) Substrate $(7 \mathrm{~g})$ and wastewater $(4 \mathrm{ml})$ were mixed and centrifuged at $8000 \mathrm{rpm}$ for $10 \mathrm{~min}$ at room temperature. The concentration of SF in the supernatant was measured.

2) The concentration of SF should not too high to avoid the density effect. When excessive high concentration of tracer added in wetland, it may sink to the bottom of the wetland if the density of tracer is higher than that of the ambient wastewater [160]. To figure out the maximum dosage of SF, SF (1 g/l) was added into $31 \mathrm{of} 1 \mathrm{mg} / \mathrm{l} \mathrm{SF}$ solution in drop-wise manner, which until the concentration of SF in the bottom was obvious higher than that in the top. The solution was stirred during addition.

3) Two types of SF loss were considered: absorption by substrate and photodegradation. Firstly, $100 \mathrm{ml} \mathrm{SF}$ of $10 \mathrm{mg} / 1$ and $2 \mathrm{ml} \mathrm{NaN}_{3}$ of $1 \mathrm{~mol} / 1$ were added into $100 \mathrm{~g}$ substrate. The mixture was shaken in dark at room temperature for 3 days. The concentration of SF in supernatant was measured after centrifugation. Secondly, $100 \mathrm{mlSF}$ of $10 \mathrm{mg} / \mathrm{l}$ were shaken in an algae incubator with light (3100 lux) for 3 days and then the concentration of SF was measured.

HRT determination in CWs. Based on the results of pre-test, $38 \mathrm{mg} \mathrm{SF}$ was injected to the inflow tubes impulsively. After injection, the samples were taken every hour (12 times in the daytime) during day 1-4 and very two hours (6 times in the daytime) during 5-16 days. The tracer response curves of SF in four CWs were simulated by equation (S8.1) by SigmaPlot software.

$C(t)=y_{0}+a \times \frac{c-1^{\frac{1 c}{c}}}{c} \times \sqrt{\frac{t-x_{0}}{b}+\frac{c-1^{\frac{1^{c-1}}{c}}}{c}} \times e \sqrt{\frac{t-x_{0}}{b}+\frac{c-1}{c}+\frac{c-1^{\frac{1^{c-1}}{c}}}{c}}$

where $\mathrm{a}, \mathrm{b}, \mathrm{c}, \mathrm{x}_{0}, \mathrm{y}_{0}$ are coefficients. The actual HRT of CWs are 2.4, 3.0, 3.3, and 2.3 for $\mathrm{CW} 1, \mathrm{CW} 2, \mathrm{CW} 3$, and CW4, respectively. 
Text S8.3 Extraction of PhACs in substrate and plant tissues

\section{Extraction of PhACs from substrate}

The extraction method was adjusted based on the description of Löffler and Ternes [240] First, $5 \mathrm{~g}$ substrate (wet weight) was centrifuged at $8000 \mathrm{rpm}$ for $10 \mathrm{~min}$ to discard pore water in the substrate. Second, Acetone/acetic acid (20/1, v/v) $(5 \mathrm{~mL})$ was added into $5 \mathrm{~g}$ substrate and the mixture was ultra-sonicated (50-60 Hz) (BRANSON 5210, Gemini BV, Netherlands) for $15 \mathrm{~min}$. After ultra-sonication, the mixture was centrifuged at $8000 \mathrm{rpm}$ for $10 \mathrm{~min}$ and supernatant was collected. Then ethyl acetate was added into the substrate, followed by ultrasonication and centrifugation. The extraction process by using ethyl acetate was repeated for 3 times. All supernatant collected was combined $(20 \mathrm{~mL}$ in total $)$ and filtrated through a glass filter (GF/F, Whatman, USA). Last, the supernatant was evaporated at $30{ }^{\circ} \mathrm{C}$ under nitrogen stream to dryness and re-constituted in $1 \mathrm{ml} \mathrm{MeOH} / \mathrm{H}_{2} \mathrm{O}(7 / 3, \mathrm{v} / \mathrm{v})$. The $1 \mathrm{ml}$ solution was centrifuged at $10000 \mathrm{rpm}$ for $10 \mathrm{~min}$ prior to UPLC analysis.

To evaluate the recovery of the method, $5 \mathrm{~g}$ substrate (wet weight) was first centrifuged discard pore water and then spiked with a mixture of seven PhACs $(10 \mathrm{ml}, 5 \mathrm{mg} / \mathrm{l} \mathrm{each})$, internal standard (dihydrocarbamazepine, $5 \mathrm{mg} / \mathrm{l})$, and $\mathrm{NaN}_{3}(0.02 \mathrm{~mol} / \mathrm{l})$. The substrate mixture was shaken in dark at $20{ }^{\circ} \mathrm{C}$ overnight. Afterwards, the substrate mixture was centrifuged at $8000 \mathrm{rpm}$ for $10 \mathrm{~min}$. The supernatant was filtrated through a glass filter for UPLC analysis and the substrate was extracted according to the method described above. Recovery of PhACs is shown in Table S8.3.

\section{Extraction of PhACs from plant tissues}

Extraction of PhACs from plant tissues were performed based on the method described by $\mathrm{He}$ et al. [158]. To evaluate the recovery of the method, $1 \mathrm{~g}$ tissues (fresh weght) of rhizoms and roots (RR), and stems and leaves (SL) were cut into $1 \mathrm{~cm}$ in serum bottles. RR and SL tissues were spiked with a mixture of seven PhACs solution (40 ml, $10 \mathrm{mg} / \mathrm{l}$ each) and internal standard (dihydrocarbamazepine, $5 \mathrm{mg} / \mathrm{l})$, and $\mathrm{NaN}_{3}(0.02 \mathrm{~mol} / \mathrm{l})$, respectively. Gas exchange was conducted to the mixture to release air in the edges of tissues so that the tissues could take up PhACs smoothly. Afterwards, the serum bottles were shaken in dark at $20{ }^{\circ} \mathrm{C}$ overnight. The supernatant was centrifuged at $10000 \mathrm{rpm}$ for $10 \mathrm{~min}$ for UPLC analysis and the tissues were extracted based on the method descried in He et al. [158]. Recovery of PhACs is shown in Table S8.3. 
Integrated constructed wetland

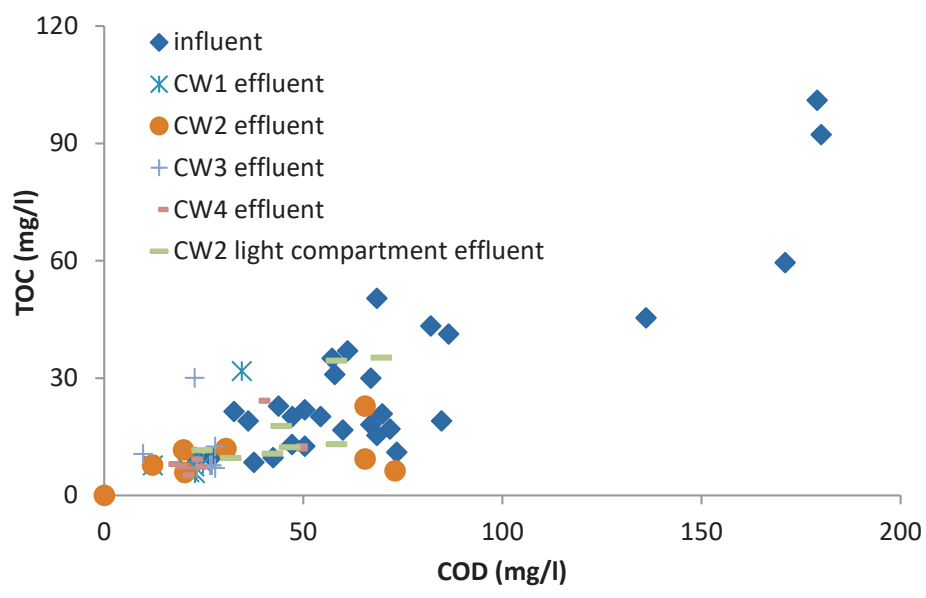

Figure S8.1 Ratio of TOC/COD of influents and effluents of mesocosm CWs $(n=8)$. 
Chapter 8
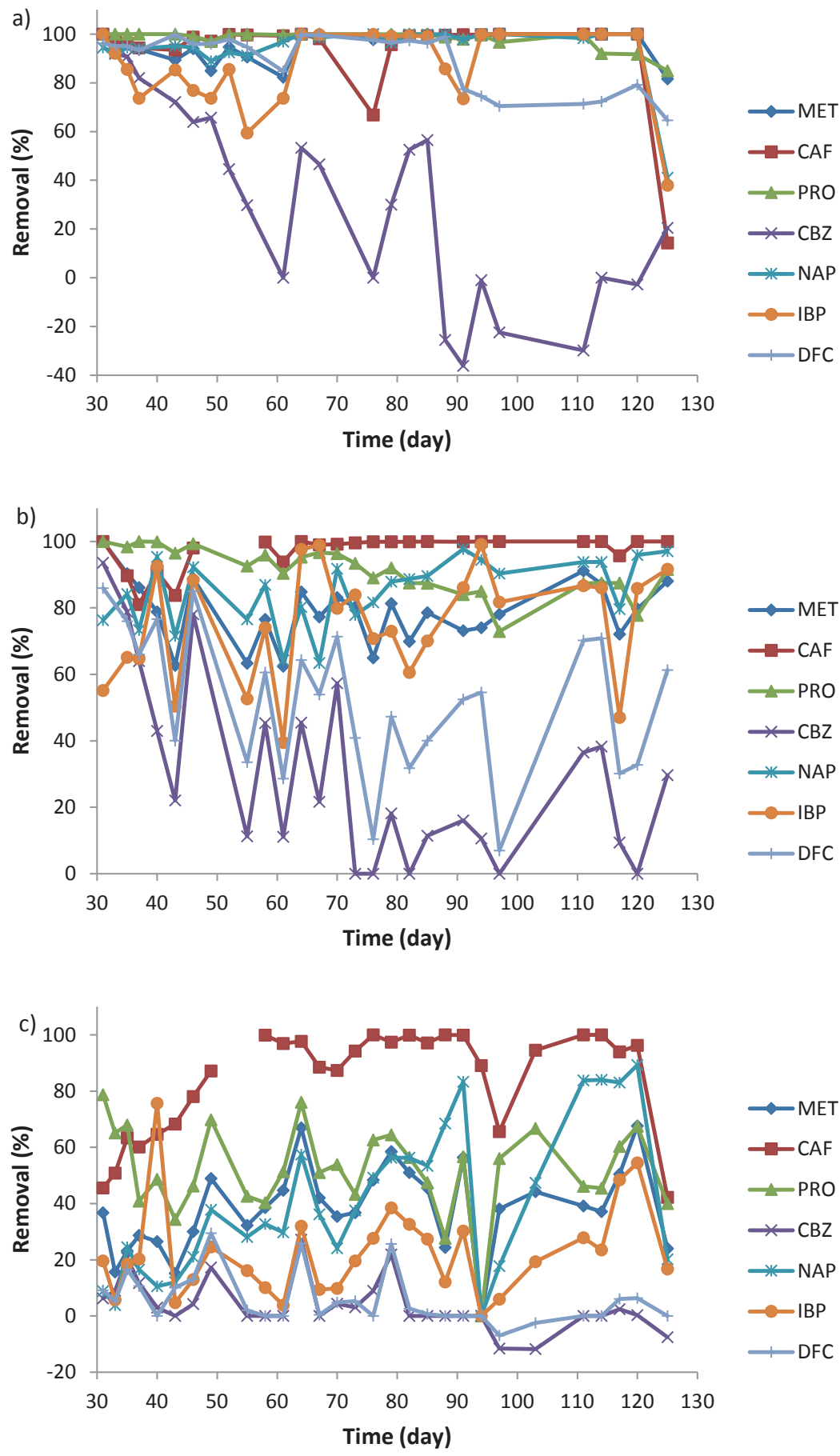

Figure S8.2 Removal of seven PhACs in mesocosm a) CW2, b) CW3, and c) CW4. 

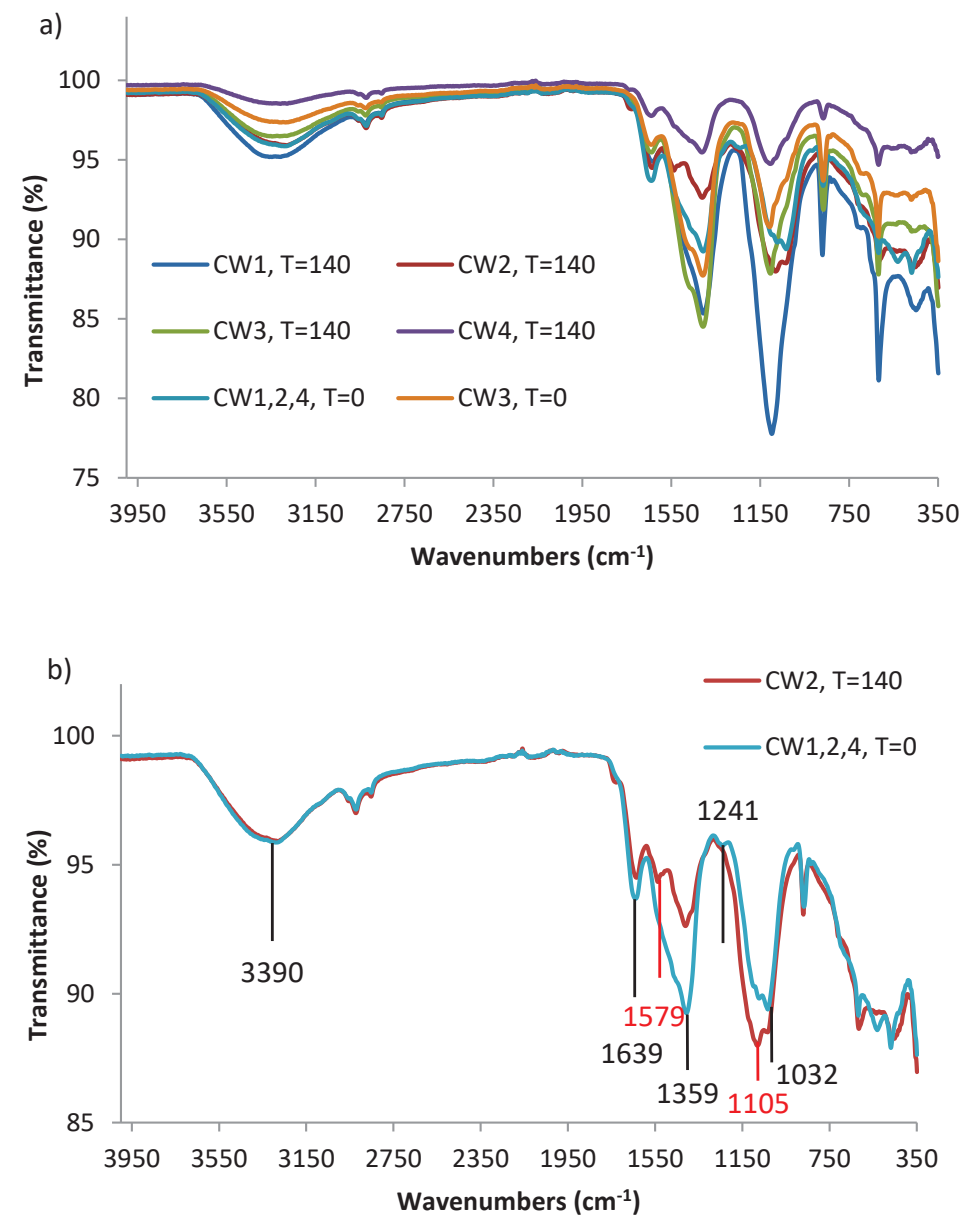

Figure S8.3 FTIR spectra of DOM at day 0 and 140. a) Except for CW1,2,4 $(\mathrm{T}=0)$ and CW2 ( $\mathrm{T}=140)$, samples from CW3 $(\mathrm{T}=0)$, CW1 $(\mathrm{T}=140), \mathrm{CW} 3(\mathrm{~T}=140), \mathrm{CW} 4(\mathrm{~T}=140)$ all have four main absorption bands: 1) $3390 \mathrm{~cm}^{-1}$ (O-H stretching of inter- and intra- molecular hydrogen bonds); 2) $1639 \mathrm{~cm}^{-1}$ (aromatic $\mathrm{C}=\mathrm{C}$ skeletal vibrations, asymmetric stretching of $\mathrm{C}=\mathrm{O}$, symmetric stretching of $\mathrm{COO}-$ ); 3 ) $1359 \mathrm{~cm}^{-1}$ (C-O asymmetric stretching of $\mathrm{COOH}$ groups); 4) $1105 \mathrm{~cm}^{-1}$ (C-O stretching of alcohols and carbohydrate). b) Samples from CW1,2,4 ( $\mathrm{T}=0$ ) contains absorption bands of 3390, 1639, 1359, 1241 , and $1032 \mathrm{~cm}^{-1} .1241 \mathrm{~cm}^{-1}$ represents for C-O stretching of aryl ethers and $1032 \mathrm{~cm}^{-1}$ represents for OH stretching of polysaccharides. Sample from CW2 ( $\mathrm{T}=140)$ contains absorption bands of 3390, $1639,1579,1359$ and $1105 \mathrm{~cm}^{-1} .1579 \mathrm{~cm}^{-1}$ represents for N-H bending amide $\Pi$ band. 
Chapter 8

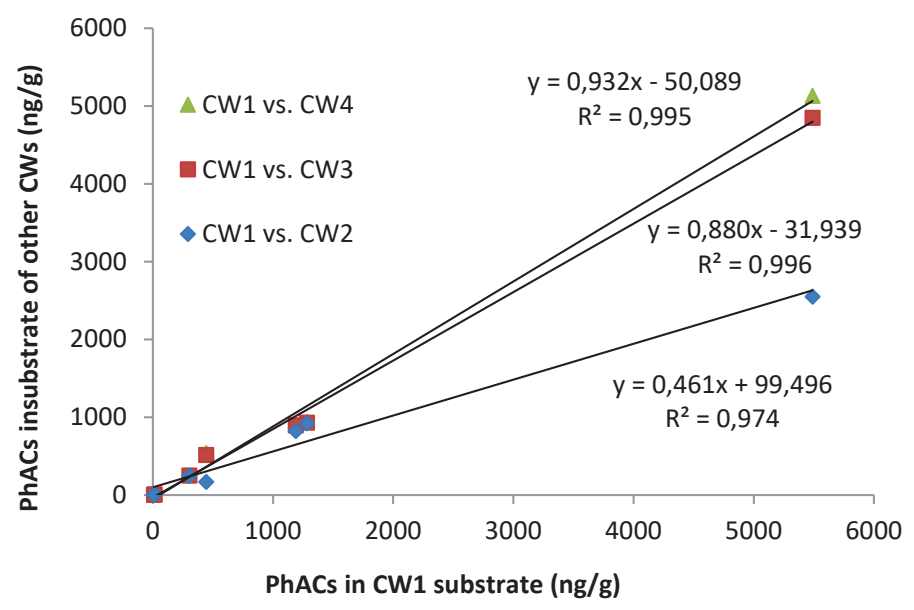

Figure S8.4 Linear relationship of PhAC distribution in the substrate of four CWs.

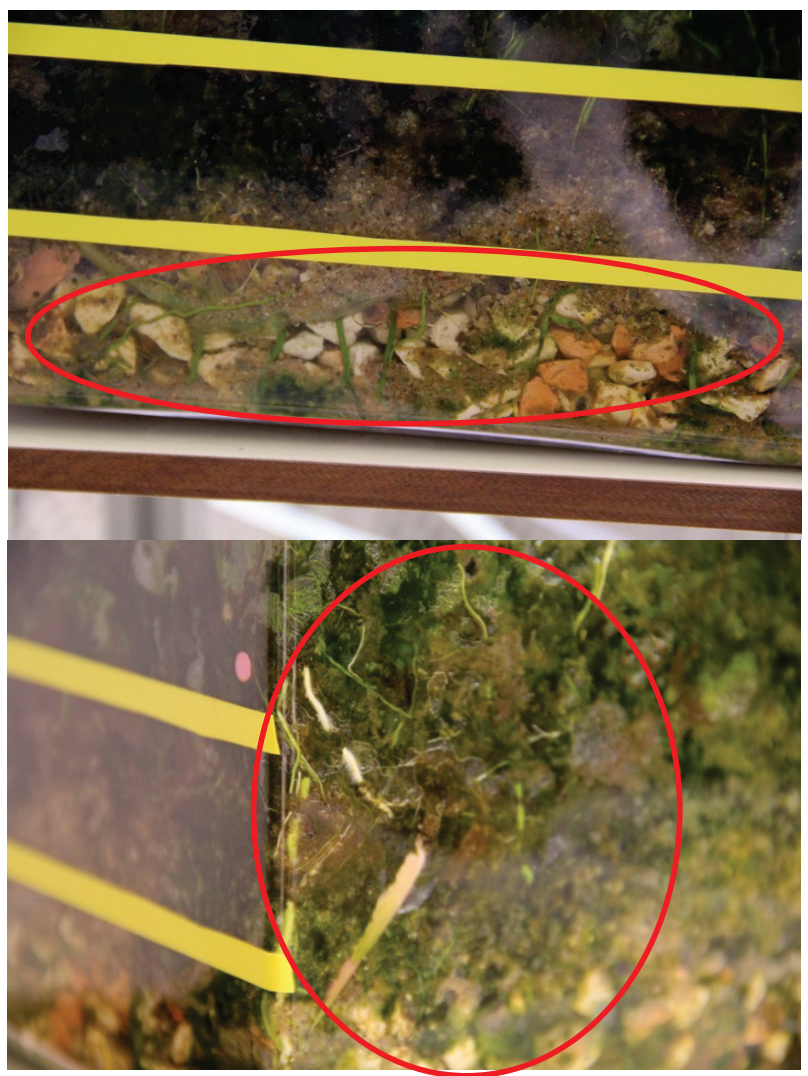

Figure S8.5 Plant roots clog up the substrate in mesocosm CWs. 


\section{Integrated constructed wetland}

Table S8.1 Concentrations and removal of COD and TOC in four CWs and the light compartment of CW2. Results are shown in average $(n=8)$.

\begin{tabular}{lcccccc}
\hline Organic matter & & CW1 & CW2 & CW3 & CW4 & CW2-light \\
\hline $\begin{array}{l}\text { Concentration } \\
(\mathrm{mg} / \mathrm{l})\end{array}$ & COD & 79.0 & 57.1 & 70.8 & 79.4 & $/$ \\
\hline & TOC & 34.8 & 20.7 & 27.3 & 34.9 & $/$ \\
Removal (\%) & COD & 66.1 & 41.6 & 66.1 & 52.6 & 0.2 \\
& TOC & 62.9 & 54.5 & 56.4 & 53.4 & -5.1 \\
\hline
\end{tabular}

Table S8.2 Peak area of hydroxyl-IBP in aqueous phase $\left(\times 10^{5} \mathrm{mAU} / \mathrm{ml}\right.$ sample $)$. Results are shown in average \pm standard deviation $(\mathrm{n}=2)$.

\begin{tabular}{llllll}
\hline CWs & CW1 & CW2 & CW3 & CW4 & CW2-light \\
\hline Influent & $1.9 \pm 0$ & $2.1 \pm 0.2$ & $1.8 \pm 0.1$ & $1.7 \pm 0.3$ & $/$ \\
Effluent & $19.7 \pm 9$ & $16.2 \pm 3.7$ & $15.6 \pm 3.9$ & $61.6 \pm 11.1$ & $32.6 \pm 6.2$ \\
\hline
\end{tabular}

Table S8.3 Recovery of PhAC extraction from substrate and plant tissues. Results are shown in average ( $\mathrm{n}=4$ for substrate extraction, $\mathrm{n}=3$ for plant extraction).

\begin{tabular}{llllllllll}
\hline Matrix & Recovery & MET & CAF & PRO & CBZ & NAP & IBP & DFC & dihyCBZ \\
\hline \multirow{2}{*}{ Substrate } & Average & 44.5 & 64.3 & 55.3 & 68.0 & 63.8 & 43.1 & 53.6 & 58.2 \\
& STD & 2.4 & 2.3 & 2.9 & 3.2 & 4.5 & 3.7 & 4.2 & 2.9 \\
\hline \multirow{2}{*}{ RR tissue } & Average & 21.4 & 17.4 & 39.1 & 20.1 & 11.7 & 2.0 & 5.4 & 10.9 \\
& STD & 5.2 & 3.4 & 5.3 & 3.0 & 2.5 & 0.2 & 1.3 & 1.1 \\
\hline \multirow{2}{*}{ SL tissue } & Average & 17.7 & 17.6 & 74.9 & 16.3 & 4.4 & 1.3 & 7.7 & 6.9 \\
& STD & 3.0 & 5.9 & 4.0 & 5.6 & 1.5 & 0.2 & 1.0 & 1.4 \\
\hline
\end{tabular}

Notes: STD represents for standard deviation. 


\section{Chapter 9}

General discussion 


\section{General discussion}

\subsection{Introduction}

The occurrence and accumulation of pharmaceutically active compounds (PhACs) in the environment has become a growing concern due to their potential threat to non-target organisms and humans. As a main barrier to prevent PhACs from entering the environment, conventional wastewater treatment plants (WWTPs) cannot remove PhACs sufficiently. To further eliminate PhACs from wastewater effluent, post-treatment processes urgently need to be established. A constructed wetland $(\mathrm{CW})$ is a promising post-treatment technique for $\mathrm{PhAC}$ removal. To date, relevant studies mainly focused on removal efficiencies of PhACs in full-scale CWs, comparison of different types of CWs in removing PhACs by lab-scale experiments, or application of CWs in removing $\mathrm{PhACs}$ from different sources of wastewater. Limited knowledge is available to understand the complex removal processes of $\mathrm{PhACs}$ that occur in CWs, mainly photodegradation, sorption, biodegradation, and phytoremediation. These removal processes are the functional treatment tools of the CW system. Only if we understand how these individual tools work, CW can be designed and operated towards optimal performance in $\mathrm{PhAC}$ removal.

In this context, this thesis investigated mechanisms of the removal processes of PhACs in CWs and interpreted corresponding outcomes into practical recommendations for application. First, $\mathrm{PhAC}$ abundance and related environmental hazards were investigated in local CWs, so that an overview of occurrence of PhACs in the wastewater effluent and their removal capacity in CWs can be obtained. Second, the CW black box was unfolded to study PhAC removal through the individual removal processes. For this purpose, various batch experiments were conducted to simulate field conditions, including using xenon lamps to mimic solar light for photodegradation studies, applying CW sediment under abiotic and biotic conditions for sorption and biodegradation studies, and cultivating wetland plants in greenhouse conditions for phytoremediation studies. Last, based on the knowledge obtained from the individual studies, all the individual removal processes were combined in mesocosm $\mathrm{CWs}$ to investigate the fate of PhACs in the whole integrated $\mathrm{CW}$ system, and to assess and verify the contribution of each process for PhAC removal in this system. Throughout the whole thesis, chemical, toxicological, phytological, and molecular methods and techniques were employed to achieve a comprehensive evaluation of $\mathrm{CW}$ performance on $\mathrm{PhAC}$ removal. 
In the following sections of this discussion chapter, outcomes originating from each chapter are integrated. Accordingly, future perspectives of PhAC removal in $\mathrm{CWs}$ are discussed including opportunities and challenges in research and application fields.

\subsection{Outcomes of the thesis}

\subsubsection{Abundance of PhACs in WWTP effluent and related environmental hazards}

Abundance of 17 PhACs in WWTP effluent was investigated by collecting samples from three Dutch WWTPs (hapter 2). These target PhACs are from various categories, including anti-inflammatory drugs, antibiotics, $\beta$-blockers, lipid regulators, psychiatric drugs, simulants, endocrine disruptors. Among the $17 \mathrm{PhACs}$, only the lipid regulator clofibric acid and two endocrine disruptors, ethinylestradiol (EE2) and estriol (E3), were not detected in WWTP effluents. Seven out of the 14 detected PhACs were present at concentrations higher than 0.1 $\mu \mathrm{g} / \mathrm{l}$. Especially, concentrations of ibuprofen (IBP) and bisphenol A reached the $\mu \mathrm{g} / 1$ level. However, concentrations of the seven PhAC we observed in the WWTP effluents exceed the guideline value $(0.1 \mu \mathrm{g} / \mathrm{l})$ adopted by toxicologists for drinking water [363].

Toxic equivalence concentrations (TEQs) showed that WWTP effluent poses environmental concern based on the evaluation by three bioassays in Chapter 2. For example, the level of estradiol equivalents (EEQ) was 0.7-1.6 pmol/1 in WWTP effluents, whereas $1.3 \mathrm{pmol} / \mathrm{l} \mathrm{EEQ}$ can already affect immature male rainbow trout to produce estrogen biomarker vitellogenin after 28 weeks of dosing [326]. In addition, most of PhACs were positively correlated to the observed toxic potency reflecting from statistical analysis (Chapter 2). Therefore, direct discharge of WWTP effluents rich in PhACs would introduce potential ecotoxicological hazards for aquatic life.

In addition to the toxicological analyses, molecular analysis outcomes also confirmed potential environmental and human health hazards of the WWTP effluent discharging (Chapter 2). Concentrations of antibiotic resistance genes (ARGs) in WWTP effluents were in the range of $6.4 \times 10^{2}-2.0 \times 10^{4}, 1.0 \times 10^{2}-1.5 \times 10^{4}$, and $9 \times 10^{0}-8.1 \times 10^{2}$ for sull, sul2 (sulfonamide resistance genes), and ermB (macrolide resistance genes), respectively. Discharge of wastewater rich in ARGs can potentially spread antibiotic resistance to microbial communities of receiving river streams, and especially low flow and small volume streams and lakes are more strongly affected [295]. In this thesis no concrete pattern of ARGs spreading can be predicted from the concentrations of related antibiotics. However, 


\section{General discussion}

concentrations of some non-antibiotic PhACs were positively correlated to ARG concentrations. This may indicate effects of PhAC emission onto $\mathrm{ARG}$ formation and dispersion. It was also suggested in previous studies that antibiotic resistance is not only caused by antibiotic resistance building selective pressure, but also through other humandriven chemical pollution influences on natural ecosystems $[4,254]$. Thus post-treatment processes urgently need to be established to eliminate PhACs in WWTP effluents, considering the hazards that PhACs deliver directly to the aquatic environment and indirectly to humans.

\subsubsection{Why choose CWs to remove PhACs?}

CWs are promising post-treatment processes as a result of their high removal of many types of PhACs, advantages of being cost-efficient and environmentally friendly, and attenuation capacity of toxicity and ARGs.

First, in CWs conditions relevant for the removal of organics and nutrients were evaluated to be functional to remove PhACs as well (Chapter 2). Field CW (Chapter 2) and mesocosm CWs (Chapter 8) functioned as treatment systems for removing organics. Accordingly, promising removal of PhACs was observed. The investigated vertical subsurface flow $\mathrm{CW}$ (VSF-CW) showed an approximate 50\% removal of 11 PhACs detected. Mesocosm CWs showed a short start-up (10 days), and high stable removal for most of the PhACs tested. Median removal of caffeine (CAF), naproxen (NAP), metoprolol (MET), propranolol (PRO), and IBP was in the range of $79-100 \%$.

Second, overall costs of CWs are generally lower than for other alternative post-treatment techniques as motivated below. Costs of CWs mainly include land cost, construction, management (basic monitoring), and maintenance (trimming and disposal of reeds annually or biennially, dredging, energy input in pumping for some CWs). The capitalized costs of CWs are approximately $€ 0.05 / \mathrm{m}^{3}$ of treated wastewater on average in the Netherlands [351]. In comparison, average costs of other techniques are several times higher at a range of $€ 0.18$ $0.43 / \mathrm{m}^{3}$, including micro/ultrafiltration, bio-filtration, flocculation filtration, coagulation, activated carbon, ion exchange, $\mathrm{UV}, \mathrm{UV} / \mathrm{H}_{2} \mathrm{O}_{2}$, and combinations of the two to three techniques [188]. The higher costs mainly result from energy usage, labour needs, chemical replacement (membrane, reagents, activated carbon, ion, UV lamps), and treatment of sludge and rinsing water. Apart from wastewater treatment in lower costs, CW systems have additional environmentally friendly and beneficial functions, e.g. forming a green element in the landscape, acting as a pool of aquaculture including plants and wild life, contributing to 
erosion control, education and training, recreation and reclamation, and water storage [100]. To some extent, CWs can yield benefits in return for their costs and those benefits are difficult to be quantified.

Third, CWs can attenuate toxic potency and ARGs from WWTP effluents and thus reduce the potential environmental hazards. Estrogenicity of wastewater effluents was 35-49\% reduced in field CWs (hapter 2). Photodegradation in an open water compartment decreased toxicity of a mixture of four PhACs from $98 \%$ to $63 \%$ inhibition of P. subcapitata, and from $67 \%$ inhibition to non-detected inhibition of A. flos-aquae (Chapter 3). In addition, positive ARG removal from WWTP effluent was observed in field CWs and mesocosm CWs. For example, absolute removal of sull, sul2, and ermB was in the range of $67-100 \%$ in two of the investigated filed CWs (Chapter 2). The mesocosm CW in Chapter 8 reduced $56 \%$ ermB in average [175].

\subsubsection{How CWs work for PhAC removal}

The principle of CWs for PhAC removal is to integrate removal processes that can occur in natural conditions and to exert them in an enhanced way in a human construction. This thesis transforms the $\mathrm{CW}$ from a black box system into a more transparent system with understanding of the individual removal processes and the interaction among these processes.

Photodegradation of PhACs driven by solar light is implemented via direct and indirect photolysis (Chapter 3). For example, direct photolysis removed $18 \%$ of IBP under simulated solar light irradiation for four days. Indirect photolysis via natural organic matter (NOM) in wastewater effluent resulted in enhanced IBP photodegradation by $50 \%$. The photodegradation process followed a pseudo-first-order. Various intermediates were formed during the photodegradation and they are in general less toxic than the parent PhACs.

Sorption mainly dominated in the start-up stage in both sediment columns (Chapter 4) and mesocosm CWs ( $\underline{\text { Chapter 8) }}$. When systems could not be further domesticated for PhAC removal, breakthrough of PhAC was observed, i.e. PRO, MET, CBZ in the sediment columns and $\mathrm{CBZ}$ in mesocosm CW. For PRO, the PhAC with the highest sorption as shown in Chapter 4, sediment sorption sites saturated after approximately 300 pore volumes. Sorption behaviour of PhACs onto $\mathrm{CW}$ sediment was shown to be more related to their molecular charge than to their hydrophobicity ( $\left.\log \mathrm{D}_{\mathrm{ow}}, \underline{\text { Chapter } 4}\right)$. The negatively charged sediment has higher potential to sorb cationic PhACs compared to anionic and neutral PhACs. 


\section{General discussion}

Moreover, sorption competition among PhACs towards sorption sites is in a relatively fixed pattern regardless of $\mathrm{PhAC}$ concentrations in the aqueous phase and treatment conditions, indicating that sorption of PhACs were reached a dynamic equilibrium in CWs with various removal processes involved (Chapter $)$.

Biodegradation of PhACs occurred under both aerobic and anaerobic conditions, which can

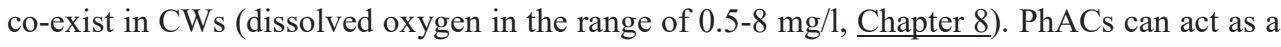
single carbon source to support microbial growth (Chapter 5). Microorganisms were found to degrade PhACs in a preferential order under aerobic batch conditions: less biodegradable PhACs were only removed once the relatively readily degradable PhACs were biodegraded (Chapter 4 and 5). Anaerobic biodegradation efficiencies of PhACs were not influenced when the PhACs were present as a single compound or as a mixture with other PhACs. However, production of different intermediates was observed under the single and mixture conditions, which suggests different biodegradation pathways in the two conditions (hapter 6). Various intermediates were formed during the anaerobic biodegradation tested and these are in general less toxic than the parent PhACs (Chapter 6). In addition to bacteria (Chapter 5), other microorganisms such as algae also grew in CWs (hapter 7 and 8). These algae are likely to contribute to biodegradation of PhACs as well (IBP, Chapter 7). Removal of PhACs in algae bioreactors was reported in the work of De Wilt et al. [92], where algae showed biodegradation of MET and paracetamol.

Occurrence of PhAC phytodegradation was confirmed during phytoremediation (Chapter 7 and 8). IBP was taken up in the roots and rhizomes (RR tissues), translocated in stems and leaves (SL tissues), and phytodegraded in plant tissues. Hydrophilic and positively charged PhACs have more potential to be taken up as 1) the uptake is a passive process and PhACs are taken up together with the aqueous phase; 2) membranes of plant cells have a negative electrical potential which favoured transport of PhACs with positive molecular charge [274]. Phytodegradation of IBP was proposed to be first catalyzed by P450 monooxygenase (P450) in the endoplasmic reticulum, then catalyzed by glycosyltransferase (GT) in Golgi, followed by further metabolism or storage in vacuoles or cell walls (Chapter 7). Moreover, P. australis can cope with IBP phytodegradation without obvious alteration of growth rate and stress enzyme activity ( $\underline{\text { Chapter 7)}}$.

In the complex $\mathrm{CW}$ systems, removal processes influence with each other. First, photodegradation can slightly increase biodegradability of wastewater (photocatalysis in 
Chapter 3), which is beneficial for the follow up biodegradation process. Phytoremediation can enhance sorption and biodegradation of PhACs, as described in Chapter 7 and 8 . Root exudates can strongly impact soil properties and thus influence sorption of neutral and ionizable organic contaminants [274]. Organic acids and proteins may promote biodegradation by acting as additional carbon sources, and enzymes in the exudates may act as catalysts in the biodegradation [313]. Additionally, roots can release oxygen and favour oxygen transport by clogging up pores in the substrate (mainly sediment), and thus enhance aerobic biodegradation.

\subsubsection{Fate of PhACs in CWs}

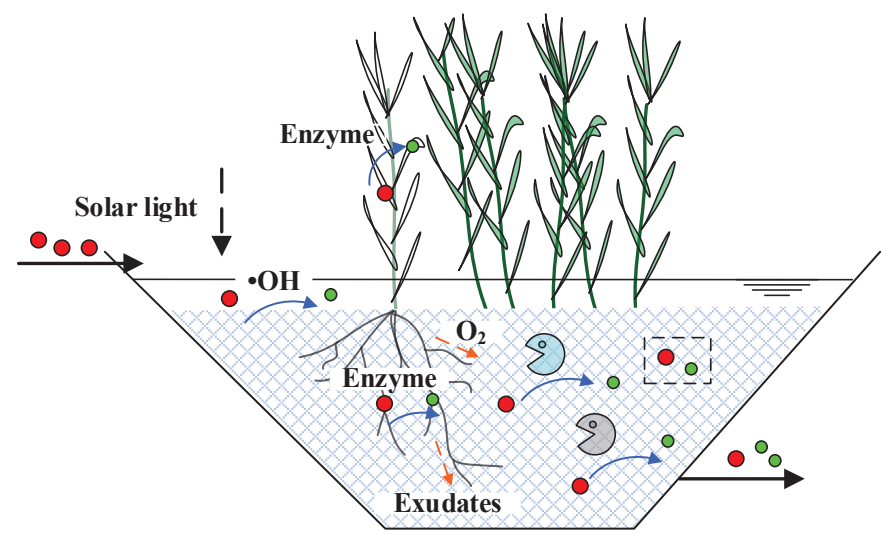

Figure 9.1 Fate of PhACs in a CW. Red dots and green dots represent for parent PhACs and their intermediates, respectively (not quantified in the figure). Part of PhACs and intermediates are retained in plant tissues and substrate (labelled in dashed rectangle). Aerobic and anaerobic microorganisms are drawn in blue and grey, respectively.

Outcomes of this thesis showed that all the removal processes are involved when $\mathrm{PhACs}$ enter CWs in wastewater effluents (Figure 9.1). Photodegradation occurs when free water surface is available with shallow depths (Chapter 3). A portion of PhACs is sorbed to the substrate,

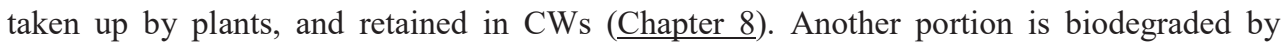

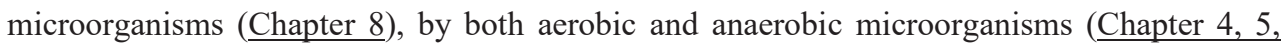
and 6), and phytodegraded via enzymatic catalysis after uptake and transport to plant tissues (Chapter 7). Residual PhACs and biodegradation intermediates flow out the CW system (Chapter 8). 


\section{General discussion}

PhACs with different physicochemical properties and biodegradability (Figure 9.2) have different removal patterns in various removal processes (Table 9.1). Specifically, PRO showed high removal potential in the individual studies of photodegradation and sorption. CAF has a high potential to be removed in CWs via both aerobic and anaerobic biodegradation. CBZ removal was the lowest compared to other six PhACs in all individual removal processes, and thus the lowest removal is expected for $\mathrm{CBZ}$ in $\mathrm{CW}$ systems having a combination of all these removal processes. Indeed, in comparison, CBZ showed the lowest removal as expected in the integrated $\mathrm{CW}$ systems (hapter 8).

Table 9.1 Removal potential of PhACs in different removal processes.

\begin{tabular}{|c|c|c|c|}
\hline Processes & Order & Influence factors & Remarks \\
\hline Photodegradation & $(\mathrm{DFC}>\mathrm{PRO})>(\mathrm{NAP}>\mathrm{MET})>(\mathrm{CAF}>\mathrm{IBP})>\mathrm{CBZ}^{\mathrm{a}}$ & $\begin{array}{l}\text { Water depth, } \\
\text { dissolved NOM }\end{array}$ & $\begin{array}{l}\text { Chapter } 3 \\
\text { and } 8\end{array}$ \\
\hline Sorption & $\mathrm{PRO}>(\mathrm{MET}>\mathrm{CAF})>\mathrm{NAP}>\left(\mathrm{IBP}, \mathrm{DFC}^{\mathrm{b}}, \mathrm{CBZ}\right)$ & $\begin{array}{l}\text { Molecular charge, } \\
\text { redox conditions, } \\
\text { solid NOM }\end{array}$ & $\underline{\text { Chapter } 4}$ \\
\hline \multirow{2}{*}{ Biodegradation } & $\begin{array}{l}\mathrm{CAF}>\mathrm{MET}>\mathrm{NAP}>\mathrm{IBP}>(\mathrm{PRO}, \mathrm{DFC}, \mathrm{CBZ}) \\
\text { aerobic }\end{array}$ & $\begin{array}{l}\text { Dissolved NOM, } \\
\text { redox conditions }\end{array}$ & $\begin{array}{l}\text { Chapter } 4 \\
\underline{\text { and } 5}\end{array}$ \\
\hline & $\begin{array}{l}(\mathrm{CAF}, \mathrm{NAP})>\left(\mathrm{MET}, \mathrm{PRO}, \mathrm{IBP}, \mathrm{DFC}^{\mathrm{b}}, \mathrm{CBZ}\right), \\
\text { anaerobic }\end{array}$ & Redox conditions & $\frac{\text { Chapter } 4}{\underline{\text { and } 6}}$ \\
\hline Phytoremediation & $\begin{array}{l}\text { Plant uptake and photodegradation. Separate } \\
\text { phytoremediation study was only conducted for } \\
\text { IBP. }\end{array}$ & $\begin{array}{l}\text { Hydrophobicity, } \\
\text { molecular charge }\end{array}$ & $\begin{array}{l}\text { Chapter } 7 \\
\underline{\text { and } 8}\end{array}$ \\
\hline
\end{tabular}

a. CBZ represents for carbamazepine; b. Diclofenac (DFC) results were not shown in Chapter 4 and 6 due to analytical issues.

In integrated $\mathrm{CW}$ systems, some removal processes play more significant roles in removing certain PhACs (Figure 9.2). When photocatalysis present, DFC was mainly removed by photodegradation. PRO removal was mainly contributed from photodegradation and sorption. Biodegradation (and/or phytodegradation) dominated the other PhACs, especially for CAF (Chapter 8). Results from the integrated studies are consistent with the outcomes obtained from the individual studies. 


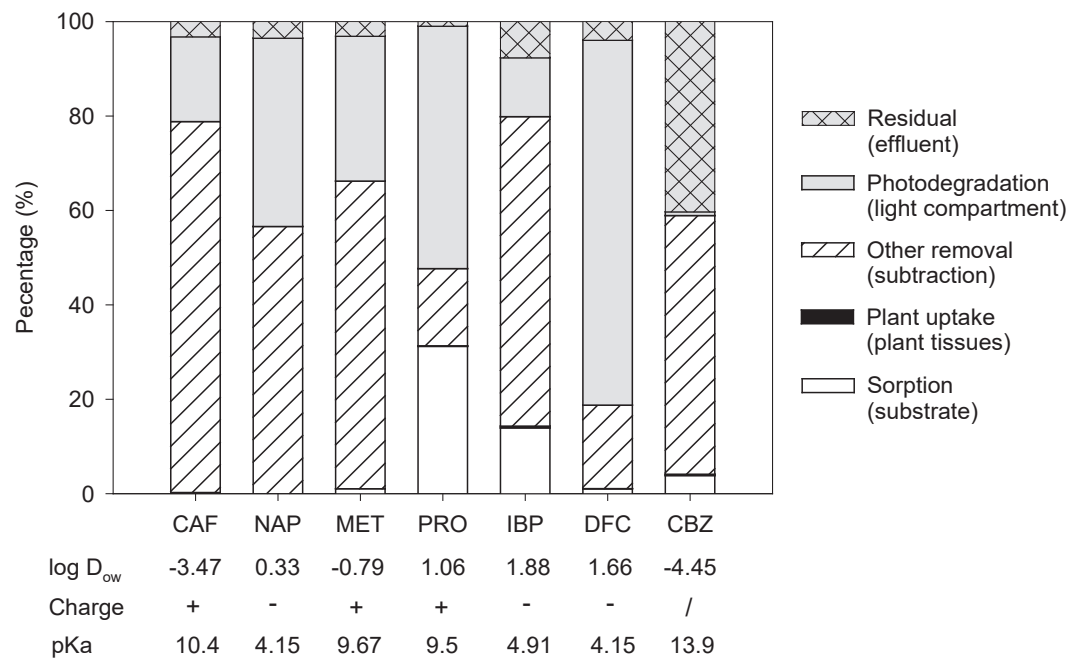

Figure 9.2 Removal of PhACs with different physicochemical properties in a $\mathrm{CW}$ (Chapter 8).

\subsubsection{Important environmental factors}

NOM and redox conditions were confirmed to be important environmental factors by this thesis. NOM in CWs originates from wastewater effluent (Chapter 3), dead plant biomass (litter, Chapter 5 and 8), and the NOM originally contained in the sediment (Chapter 8). This thesis demonstrated that NOM can affect photodegradation, sorption, and biodegradation of $\mathrm{PhACs}$ in CWs with complex mechanisms. First of all, dissolved NOM in wastewater effluent can enhance photodegradation of PhACs by producing triplet states of NOM $\left({ }^{3} \mathrm{NOM}^{*}\right)$ and further to $\bullet \mathrm{OH}$. These radicals can degrade PhACs, an indirect photolysis process. In contrast, dissolved NOM also inhibited photodegradation, possibly because dissolved NOM reforms the oxidation intermediates of PhACs into parent compounds (Chapter 3). Second, solid NOM in $\mathrm{CW}$ sediment may promote sorption of NAP in batch experiments of Chapter 4, as NAP is more favored to partition to NOM rather than the inorganic surface of the sediment [251]. However, effect of solid NOM on sorption was not observed in Chapter 8 . The inconsistency may be explained by the different sources (composition) of NOM in these two chapters. Last, dissolved NOM extracted from litter enhanced PhAC biodegradation, potentially by either supporting growth of biomass capable of degrading PhACs, acting as structural analogues, or acting as electron shuttles to stimulate microorganisms for metabolism or co-metabolism of PhACs ( $\underline{\text { Chapter 5) }}$. Similarly, the enhancement of solid NOM on PhAC removal through litter addition was also observed in Chapter 8 for PhAC biodegradation. However, extracted 


\section{General discussion}

dissolved NOM can also inhibit biodegradation of readily biodegradable $\mathrm{PhACs}$ through competition (hapter 5).

Redox conditions, dominated by the availability of various electron acceptors, influence $\mathrm{PhAC}$ removal. Aerobic, nitrate reducing, sulfate reducing, and methanogenic conditions coexist in CWs [334]. Both batch and column experiments as reported in this thesis showed that aerobic conditions favored $\mathrm{PhAC}$ biodegradation compared to the anaerobic conditions investigated (Chapter 4). Nitrification occurs when there is sufficient ammonium and oxygen. As indicated in Chapter 5, biodegradation of PhACs with linear alkyl carbon structure can be enhanced under nitrifying conditions, likely resulting from co-metabolism driven by ammonia oxidizing bacteria. Moreover, nitrification can even mineralize NOM and PhACs. Interestingly, MET biodegradation was lower under nitrifying conditions, a phenomenon which has yet to be fully mechanistically explained. To date, no literature demonstrates inhibition of PhAC degradation by nitrifying cultures.

Among the nitrate reducing, sulfate reducing, and methanogenic conditions, no specific redox condition was found to result in a more effective overall biodegradation of $\mathrm{PhACs}$ (Chapter 4 ). Similarly, no obvious difference in removal efficiencies was observed in various anaerobic conditions before transfer for microbial enrichment in Chapter 6. However, differences appeared during and after transfer. Under nitrate reducing conditions, biodegradation capacity of CAF and NAP was lost during transfer and did not recover thereafter. In terms of maintenance of biodegradation capacity, microorganisms capable of biodegrading $\mathrm{PhACs}$ were more robust under sulfate reducing and methanogenic conditions. After transfer, methanogenic conditions showed faster biodegradation of CAF compared to sulfate conditions. Additionally, different intermediates were found under these two redox conditions, which indicate different biodegradation pathways by microbial communities under each specific redox conditions. As also indicated by different biodegradation pathways observed in the single and mixed studies in section 9.2.3, for future research investigation of intermediate production should be as important as the investigation of removal of parent PhACs.

An unexpected phenomenon was observed when studying the various anaerobic conditions. That is, specific anaerobic conditions can affect sorption capacity of $\mathrm{CW}$ sediment toward

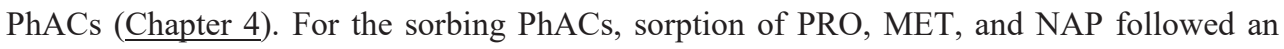
order of nitrate reducing $<$ methanogenic $<$ sulfate reducing. In contrast, CAF followed a different order, that is nitrate reducing $<$ sulfate reducing $<$ methanogenic conditions. It seems 
that nitrate reducing conditions are not favorable for $\mathrm{PhAC}$ sorption. The effect of redox condition on sorption behavior of PhACs onto sediment has not been described before, but interesting questions arise about the underlying mechanism warranting further research in the future.

\subsubsection{Enhancement of CW capacity for PhAC removal}

This thesis proposes several ways to enhance capacity of CWs for PhAC removal through strengthening individual removal processes and operational parameters. Photodegradation of PhACs occurred under experimental conditions with light intensity comparable with the light intensity in the Netherlands ( $\underline{\text { Chapter } 3}$ ). In the field, attenuation of PhACs was found in open waters, which indicates the role of photodegradation (Chapter 2). In practice, free water surface is only available in surface flow CWs (SF-CWs) and the surface is normally shaded by plants. Therefore, an open water compartment can be integrated with a $\mathrm{CW}$ as a means of photodegradation enhancement, as reported previous by Jasper and Sedlak [185]. Reflecting from both experimental and modelling results in Chapter 3 , direct photolysis capacity significantly decreased with water depth when the depth was less than $0.1 \mathrm{~m}$. Photolysis was negligible at depths above $0.1 \mathrm{~m}$. Thus, the open water compartment should be a shallow unit.

To further increase the photodegradation potential, catalysts can be applied, as we successfully performed in Chapter 3 and 8 . Compared to photolysis, photocatalysis driven by immobilizing $\mathrm{TiO}_{2}$ was faster and more complete (Chapter 3). In addition, photocatalysis led to attenuation of PhAC toxicity and increase of wastewater biodegradability (Chapter 3). By establishing the open shallow photocatalysis compartment prior to a $\mathrm{CW}, \mathrm{PhACs}$ can be highly removed (Chapter 8). The high removal of PhACs during pre-photocatalysis futher reduces the burden of $\mathrm{PhAC}$ accumulation in the substrate and plants in the following plant compartment. The added value of photocatalysis application might be questioned as dissolved NOM in wastewater seemed to act as a "catalyst" as well (Chapter 3). However, dissolved NOM composition in wastewater effluent varies greatly due to the fluctuating quality of wastewater influent. Inhibition of photodegradation may occur due to dissolved NOM by quenching $\cdot \mathrm{OH}$, screening photos, and/or reacting with oxidized PhACs (Chapter 3 ). Therefore, photocatalysis is a practical option to maintain high and stable performance of photodegradation.

Sorption of certain PhACs in CWs might be enhanced by additional NOM (NAP, Chapter 4). To this end, a feasible way is to partially dispose plant tissues directly in CWs after trimming. 


\section{General discussion}

One concern of direct plant disposal is that accumulated contaminants including PhACs in the plant tissues may release back to the substrate. To some extent, this concern is not necessary at least for PhACs, because plants not only take up PhACs but also phytodegrade them. In fact, less than $1 \%$ of the total PhAC mass entering CWs accumulated in plant tissues (Chapter 8). Another concern is that the litter may consume oxygen resulting in less aerobic biodegradation. However, according to the observations in this thesis, in addition to enhanced sorption, litter addition can offer added value in promoting PhAC biodegradation.

Favorable biodegradation conditions can be manipulated by litter addition (Chapter 5 and 8), microbial enrichment (Chapter 5 and 6), and improvement of redox conditions (Chapter 4 and 5). Addition of litter led to high biodegradation of PhACs in the thesis, as described in section 9.2.5. Furthermore, considering the significant role of $\mathrm{NOM}$ for $\mathrm{PhAC}$ removal, litter addition can also replenish release of NOM in CWs that was observed in Chapter 8. Microbial enrichment through re-spiking or transfer accelerated aerobic and anaerobic biodegradation of PhACs (Figure 9.3). Enrichment shortened the period required to achieve almost completed PhAC removal (>95\%). Aerobic conditions and nitrifying conditions have superiority for $\mathrm{PhAC}$ biodegradation (Chapter 4 and 5). To this aim, aeration is suggested to be improved in CWs to increase activity of aerobic heterotrophs and nitrifiers. Ammonium concentration is normally low in the WWTP effluent due to regulation, resulting in limited nitrification in CWs as post-treatment processes. To maintain successful nitrification in CWs, a sub-stream of wastewater influent of WWTPs rich in ammonium could be introduced to CWs to support biodegradation of PhACs of interests. 


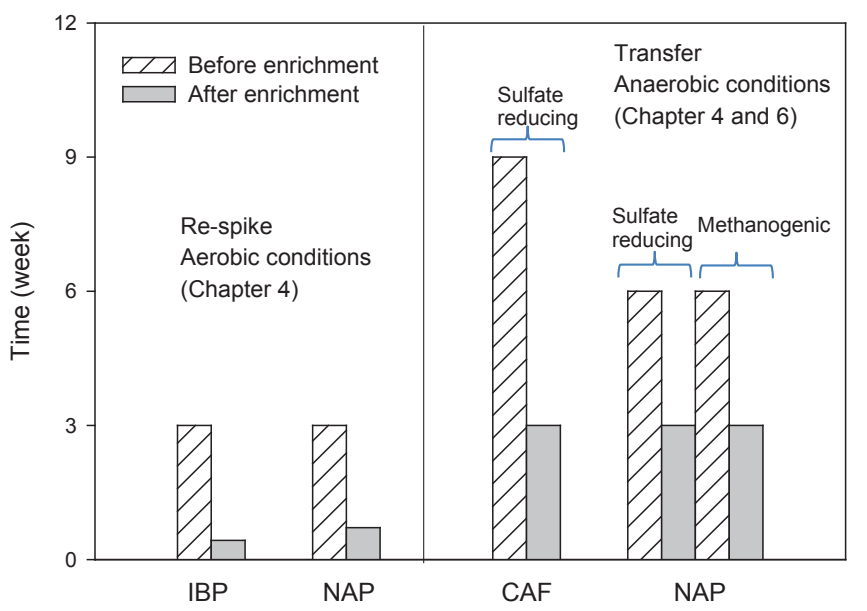

Figure 9.3 Time required for almost complete removal (> 95\%) of PhACs before and after microbial enrichment (re-spiking and transfer). Before transfer, complete removal comprises of both sorption and biodegradation, while after transfer removal was only by biodegradation.

Phytoremediation plays an essential role in CWs for PhAC removal directly by uptake and phytodegradation, and indirectly by stimulating sorption and biodegradation (Chapter 8). Optimizing vegetation can be considered as a strategy to maximize plant performance, including selection of plant species and designing plant density. For example, P. australis was found to be the best candidate for removing two pesticides, outperforming other commonly applied wetland plant species, including Typha latifolia, Iris pseudacorus and Juncus effuses [243]. A CW with a densely planted Typha latifolia $\left(40\right.$ plants $\left./ \mathrm{m}^{2}\right)$ showed a faster removal of chloroform (a disinfection by-product), compared to a sparsely planted CW (22 plants $/ \mathrm{m}^{2}$ ) [69]. Thus, optimization of vegetation might be another approach to enhance $\mathrm{PhAC}$ removal in CWs.

Apart from enhancing CW capacity by optimizing operational parameters such as aeration, feeding regime, and vegetation for stimulating specific removal processes as described, this thesis also indicated enhancement of the overall removal capacity through optimization of other operational parameters including configuration and hydraulic loading rate (HLR). Significantly higher PhAC removal was found in a VSF-CW compared with other two SFCWs (Chapter 2). VSF-CWs can achieve a better oxygenation and possess a superior rhizosphere effect in rhizodegradation and sorption [259, 400]. The better performance of the VSF-CW may also relate to its lower HLR $(17.6 \mathrm{~cm} / \mathrm{d})$, which leads to long contact and 


\section{General discussion}

interaction between contaminants and removal matrices. In our lab-scale horizontal subsurface flow CWs (HSF-CWs, HLR $=5 \mathrm{~cm} / \mathrm{d}$ ), high $\mathrm{PhAC}$ removal performance was also observed (Chapter 8). According to a large database review, it is complex to determine which configuration is the best, as some PhACs can be removed better in one configuration than the others [362]. However, certain configuration can be selected for specific PhACs of interests [362].

In summary, this thesis raised a new concept of $\mathrm{CW}$ with a pre-photocatalysis compartment to maximize its photodegradation function. Other ways to enhance the $\mathrm{CW}$ capacity towards PhAC removal are summarized in Figure 9.4, including litter addition, microbial enrichment, improvement of aeration, optimization of vegetation, import of ammonium stream, and optimization of operational parameters e.g. HLR.

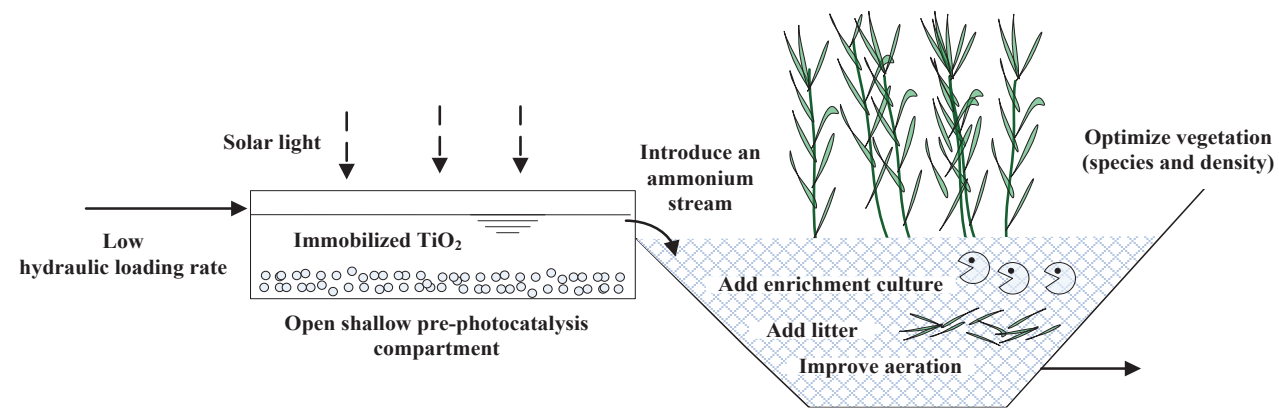

Figure 9.4 Proposed new concept of $\mathrm{CW}$ configuration and ways to enhance $\mathrm{CW}$ capacity for PhAC removal. In the figure the plant compartment is a HSF-CW as an example.

\subsection{Future perspectives}

\subsubsection{Opportunities and challenges in research}

Despite this thesis's in-depth investigation into individual removal processes of PhACs and their integration in CWs, the complete fate of PhAC molecules in CWs in not yet transparent. First, "the other removal processes" indicated in Figure 9.2 is a combination of biodegradation, phytodegradation, insignificant photodegradation, and possibly irreversible sorption to soil. A portion of these removal processes cannot be quantified. The mass balance estimated only relies on concentration analysis of parent PhACs. Only intermediates of IBP were investigated and not quantified. Actually, quantification of intermediates will not offer a clear clue to distinguish the proportion, as hydroxyl-IBP can be produced by all those 
degradation processes (Chapter 7 and 8). Namely, hydroxyl-IBP detected in plant tissues can be from uptake of hydroxyl-IBP formed via either photodegradation or biodegradation. There is a potential to distinguish contribution of various degradation processes to the production of intermediates by using compound specific stable isotope analysis (CSIA). Chemical bonds in molecules formed by light isotopes react kinetically different with bonds with heavier isotopes [110]. Extent of isotope fractionation relies on the type of degradation process [172]. Therefore, CSIA may indicate distinct degradation processes. For example, direct photolysis of sulfamethoxazole was distinguished from its biodegradation by a pure culture using CSIA [38].

Second, in this thesis only IBP intermediates were investigated while intermediates of other target $\mathrm{PhACs}$ are unknown. Their degradation pathways can be very complex, as indicated by numerous chromatography peaks of intermediates observed in Chapter 3 and 6 . Additionally, some of these produced intermediates were further transformed with time. To get a closer look at the produced intermediates and obtain a turnover mass balance of PhACs in CWs, a radioactive isotope analysis (e.g. labelled with ${ }^{14} \mathrm{C}$ ) can be implemented. Radioactivity of intermediates can be quantified and intermediate fractions with different radio-chromatogram can be collected and further qualified. This kind of isotope analysis has been studied in the field of herbicides [219,371], brominated flame retardants [220], but much less in the fate of PhACs.

Third, environmental hazards of the intermediates might be of concern. As observed in Chapter 6 , toxic intermediates were produced during incubation, although the overall toxic potency decreased after biodegradation. Therefore, it is crucial to have a thorough assessment of potential toxic intermediates. Primarily, the potential toxic intermediates can be screened as we did by using statistical tools to explore the correlation between chemical and toxicological outcomes (Chapter 6). After narrowing down the range of toxicants, effect-directed analysis (EDA) can be employed to identify the hazardous intermediates. EDA is a combination of bioassays, fractionation and chemical analysis (Brack, 2003). 2-[2-(chlorophenyl) amino] benzaldehyde has been identified as a photolysis intermediate of DFC by using EDA. This intermediate was confirmed to be more acutely toxic than DFC to the tested algae acceptor [323].

Last, PhAC biodegradation in CWs results from both aerobic and anaerobic microorganisms (Chapter 4, 5, and 6). However, it is unknown which group of microorganisms contributed to 


\section{General discussion}

the biodegradation. Litter addition enhanced PhAC biodegradation in CWs (Chapter 5 and 8), but it is unclear how the litter stimulated microorganisms to achieve a higher $\mathrm{PhAC}$ removal performance. It is a complex topic to explore the inter-relationship between $\mathrm{PhAC}$ removal and contributing microbial communities, and the effect of manipulation factors on microbial communities. However, with development of molecular analysis and more research outcomes as references, it is possible to gain more knowledge on this topic. A high-throughput pyrosequencing analysis has been used to link some aerobic and anaerobic microbial species to metabolic and co-metabolic biodegradation of IBP [225]. In addition, a combination of high-throughput pyrosequencing analysis and statistical analysis has proven that litter addition significantly influenced microbial communities due to alteration of carbon content and $\mathrm{pH}$ in the substrate instead of through alteration of other parameters, e.g. nutrients, oxidation/reduction potential [72]. Planted CWs with litter addition was found to own more organic matter degraders, nitrifying and denitrifying bacteria, which may favor removal of organic contaminants [72]. Additionally, the role of another microorganisms, algae, that grow pervasively on the substrate of CWs (Chapter 8) in PhAC biodegradation can be explored further.

This thesis shows the significant roles of photodegradation and biodegradation in CWs for breaking down PhACs. Enhancement of these two processes can be implemented by prephotocatalysis and microbial enrichment. For application purposes, further studies are required such as optimization of the catalyst and evaluation of applying the enrichment culture. $\mathrm{TiO}_{2}$ was immobilized on sand in this thesis. Other materials also have been to studied to support $\mathrm{TiO}_{2}$ for photocatalysis, such as quartz, silica, ceramics, zeolites, and pumice stones [357]. Especially, the pumice stones (porous lava) with high porosity may create larger specific surface area for a more stable immobilization.

With an enrichment culture, it is possible to perform bioaugmentation to enhance biodegradation of PhACs in CWs. Bioaugmentation is an effective alternative to provide microorganisms with specific functions in sufficient abundances for bioremediation. It is conducted by adding a pre-adapted culture (pure bacterial strain or consortium) to the initial indigenous microbial community [377]. The bioaugmentation strategy has been studied in CWs for improving nitrogen removal in the last decade [272] and recently for pesticides [411], but is nearly unknown for PhACs. For application, the possible capacity loss of activity during transfer ( $\underline{\text { Chapter } 6}$ ) needs to be avoided, possibly by addition of solid materials as carriers, e.g. beads and vermiculite [213]. Certainly, during bioaugmentation, survival of the 
added microorganisms might be threatened by various abiotic and biotic conditions [411], which needs to be further assessed.

\subsubsection{Opportunities and challenges in application}

$\mathrm{CW}$ is an economical and environmentally friendly technique. Due to numerous advantages as described in 9.2.2, CWs have been used and developed world widely. In general, the removal efficiencies of organics and nutrients are relatively sufficient (Table 9.2). Considering the positive correlation between organics and nutrients removal and $\mathrm{PhAC}$ removal (Chapter 2), comparable $\mathrm{PhAC}$ removal is also expected in these CWs. To be specific, $\mathrm{CW}$ application in the Netherlands and China is compared here. The first CW applied in the Netherlands was in 1985. The amount of CWs has been developed from 15 in 2012 to currently 43 (Climatescan, open source database). Most of them are operated in SF mode [351]. The Dutch CWs are mainly constructed to remove nutrients and pathogens, and supply water for agriculture, nature, or recreation [351]. Learning from Dutch waterboards, a new application direction of CWs is to remove micropollutants including PhACs, especially for CWs that will be renovated in the near future. In comparison, CW was introduced to China in 1987. Until 2006, more than $200 \mathrm{CWs}$ were constructed, among which 16\% are SF-CWs, 29\% are HSF-CWs, and 54\% are VF-CWs or combined CW systems [234]. CWs in China are mostly applied to treat domestic, industrial, and agricultural wastewater [405]. There is growing recognition in China that CWs offer a viable alternative, or at least a supplementary technique for wastewater treatment. Especially the Chinese policy makers are encouraging CW application in rural areas of China. Therefore, a large market of construction of CWs is foreseen in China in the next decades.

However, land requirement might be a limiting factor for a broader CW application, especially in regions with scarce land resources and dense population. By enhancing CW performance, capacity is increased and this limitation can be reduced to some extent. Therefore, a suite of robust and sustainable CW techniques with enhanced removal capacity, and minimized land area footprint is required for successful long-term application either in renovation or in new construction. 


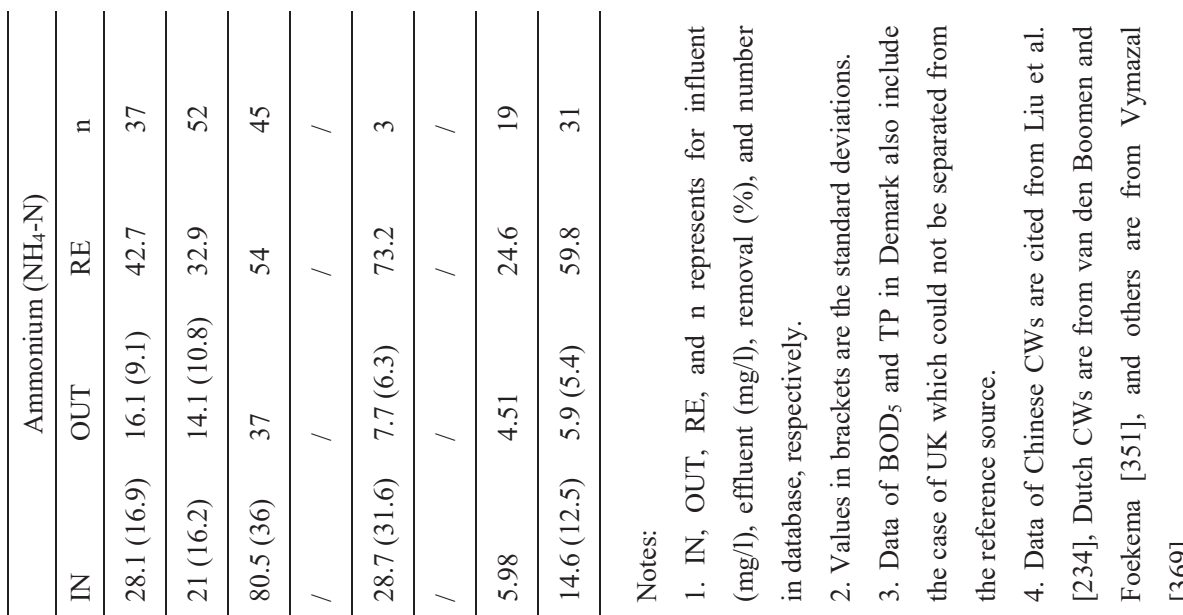

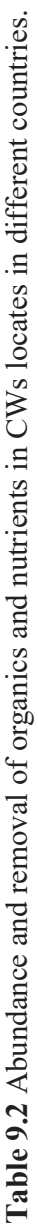

ชิ

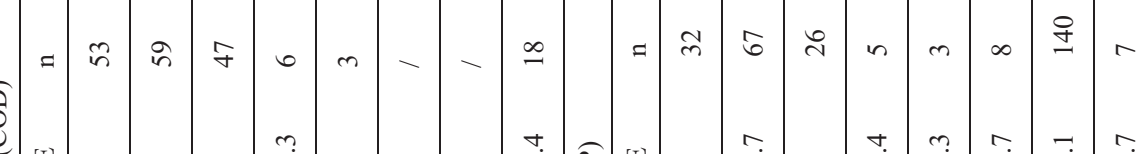

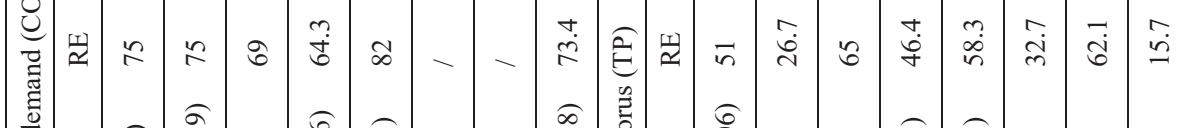

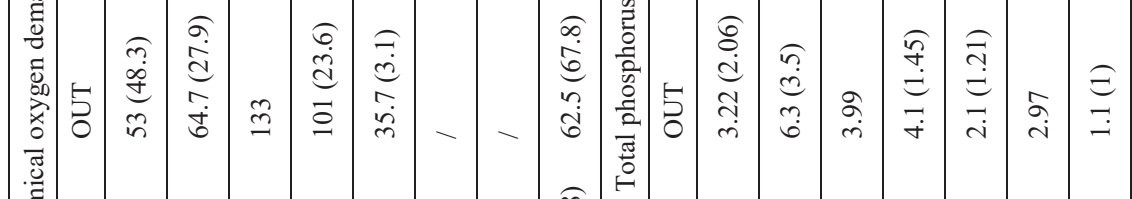
ปี

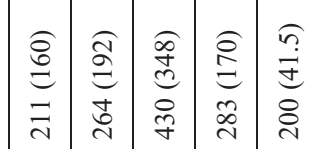

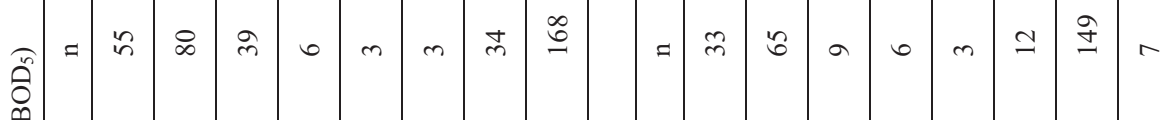

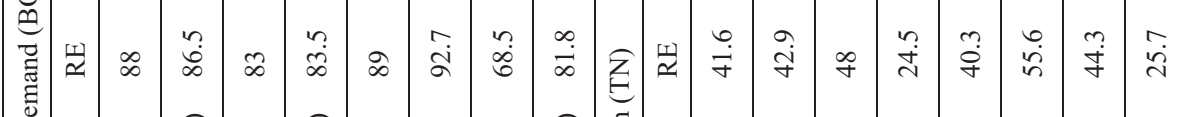

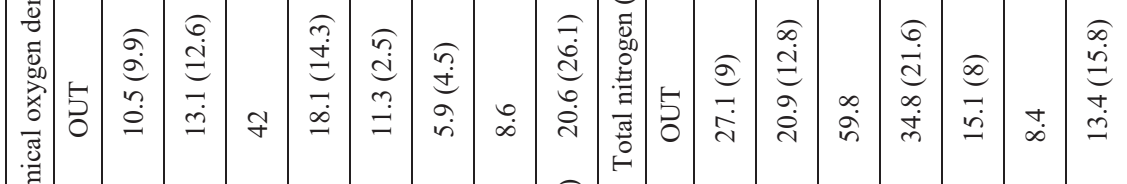

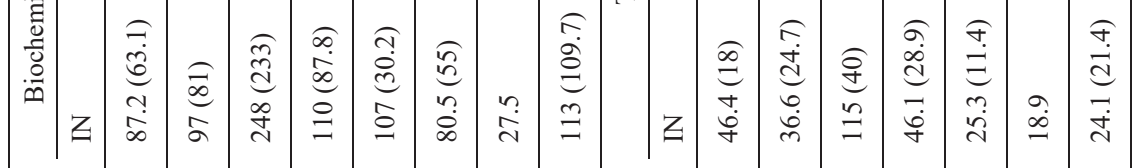

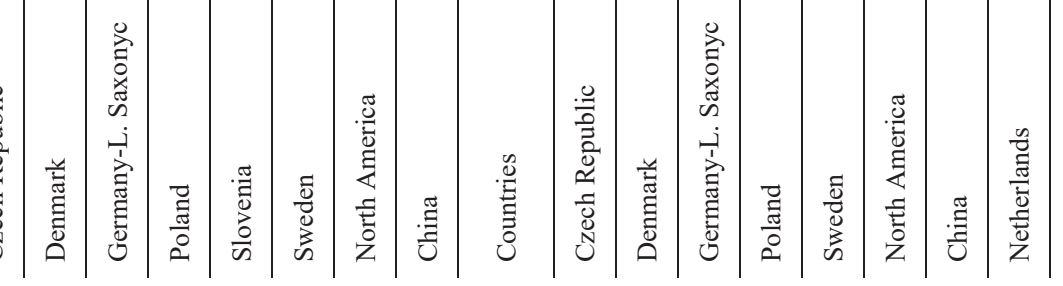


This thesis provided suggestions about enhancement through pre-photocatalysis, litter addition and improvement of aeration, all of which have the potential to be applied in the near future. The immobilized $\mathrm{TiO}_{2}$ applied in this thesis is a robust catalyst. During 125 days of operation, notable algae growth occurred in the light compartment that could have attenuated the irradiation, but no negative effect was observed in PhAC removal (Chapter 8). By immobilizing $\mathrm{TiO}_{2}$ on sand, ecotoxicological hazards of $\mathrm{TiO}_{2}$ nanoparticles to aquatic life could be eliminated. The estimated production cost of the immobilized $\mathrm{TiO}_{2}$ based on experimental grade chemicals is $€ 472$ per $2.64 \mathrm{~kg}$ for $1 \mathrm{~m}^{2}$ use in CWs to achieve comparable high photodegradation performance as in this thesis (Chapter 8). Assuming this catalyst is applied in the three field CWs in Chapter 2, the extra land requirement for the prephotocatalysis compartment will be 1833, 598, and $2083 \mathrm{~m}^{2}$ for CW-L, CW-H, and CW-K, respectively. That will be $10 \%, 9 \%$, and $24 \%$ of the current surface area of these three CWs. For future application, the long-term operation of the catalyst should be assessed. To date, there is no firm report to demonstrate the life-time of $\mathrm{TiO}_{2}$ before deactivation. It is reported that $\mathrm{TiO}_{2}$ can be reused by separating them with sedimentation particles and heating at $200{ }^{\circ} \mathrm{C}$ for re-activation $[122,357]$.

Litter addition has been recently studied for nitrate and sulfate removal in CWs [70, 71, 73], but not yet for PhACs. For application in enhancing PhAC removal, litter is suggested to be added in an appropriate amount. Excessive organic matter in CWs may lead to a series of negative effects on pollutant removal, such as decrease of hydraulic conductivity, hydraulic malfunction, block of oxygen transport, and reduction of biodegradation [272]. Therefore, the appropriate amount of litter addition must be determined.

For improving aeration, artificial aeration has been studied to improve redox conditions in VSF-CWs [116]. Compared to artificial aeration, a more economical way is to create passive aeration via a tower cascade flow or a reciprocating/tidal flow in CWs. The tower cascade using gravitational energy drives flow from upper layer to lower layer and stimulates oxygen transport [389]. The tidal flow design can produce fluctuating redox conditions through several cycles of flood and drain daily [176]. However, these aeration innovations will increase the lifecycle cost of CWs, and thus a balance of investment and efficiency needs to be determined.

Apart from the suggestions indicated from this thesis, there are also other ways to improve the capacity of $\mathrm{CWs}$ for PhAC removal, such as selection of substrate materials and 
optimization of feeding mode. Although there are no fixed criteria for optimal CW designing and operation, some recommended parameters of CWs for wastewater treatment are available [378]. In summary, enhancement of CW capacity towards PhAC removal is feasible and promising. Reducing the footprint in design studies may widen further the application potential of $\mathrm{CW}$. With the development of fundamental research and increase of market demand for removing the emergent PhACs, implementation of this natural and sustainable CW technology will increase in the coming decades. 
Bibliography 


\section{Bibliography}

1. Accinelli C., Saccà M.L., Mencarelli M., Vicari A. Application of bioplastic moving bed biofilm carriers for the removal of synthetic pollutants from wastewater, Bioresour. Technol., 120 (2012) 180-186.

2. Agüera A., Perez Estrada L.A., Ferrer I., Thurman E.M., Malato S., Fernandez-Alba A.R. Application of time-of-flight mass spectrometry to the analysis of phototransformation products of diclofenac in water under natural sunlight, J. Mass Spectrom., 40 (2005) 908-915.

3. Ahmed M.B., Zhou J.L., Ngo H.H., Guo W., Thomaidis N.S., Xu J. Progress in the biological and chemical treatment technologies for emerging contaminant removal from wastewater: A critical review, J. Hazard. Mater., 323, Part A (2017) 274-298.

4. Alonso A., Sánchez P., Martínez J.L. Environmental selection of antibiotic resistance genes, Environ. Microbiol., 3 (2001) 1-9.

5. Alvarino T., Nastold P., Suarez S., Omil F., Corvini P.F.X., Bouju H. Role of biotransformation, sorption and mineralization of 14C-labelled sulfamethoxazole under different redox conditions, Sci. Total Environ., 542, Part A (2016) 706-715.

6. Amorim C.L., Moreira I.S., Maia A.S., Tiritan M.E., Castro P.M.L. Biodegradation of ofloxacin, norfloxacin, and ciprofloxacin as single and mixed substrates by Labrys portucalensis F11, Appl. Microbiol. Biotechnol., 98 (2014) 3181-3190.

7. Anderson J., Carlson J., Low J., Challis J., Wong C., Knapp C., Hanson M. Performance of a constructed wetland in Grand Marais, Manitoba, Canada: Removal of nutrients, pharmaceuticals, and antibiotic resistance genes from municipal wastewater, Chemistry Central Journal, 7 (2013) 54.

8. Anderson J.C., Carlson J.C., Low J.E., Challis J.K., Wong C.S., Knapp C.W., Hanson M.L. Performance of a constructed wetland in Grand Marais, Manitoba, Canada: Removal of nutrients, pharmaceuticals, and antibiotic resistance genes from municipal wastewater, Chem. Cent. J., 7 (2013) 54.

9. Andreozzi R., Marotta R., Pinto G., Pollio A. Carbamazepine in water: persistence in the environment, ozonation treatment and preliminary assessment on algal toxicity, Water Res., 36 (2002) 2869-2877.

10. Andreozzi R., Raffaele M., Nicklas P. Pharmaceuticals in STP effluents and their solar photodegradation in aquatic environment, Chemosphere, 50 (2003) 1319-1330.

11. Antunes P., Machado J., Sousa J.C., Peixe L. Dissemination of sulfonamide resistance genes (sul1, sul2, and sul3) in Portuguese Salmonella enterica strains and relation with integrons, Antimicrob. Agents Chemother., 49 (2005) 836-839.

12. Atashgahi S., Aydin R., Dimitrov M.R., Sipkema D., Hamonts K., Lahti L., Maphosa F., Kruse T., Saccenti E., Springael D., Dejonghe W., Smidt H. Impact of a wastewater treatment plant on microbial community composition and function in a hyporheic zone of a eutrophic river, Sci. Rep., 5 (2015) 17284.

13. Auerbach E.A., Seyfried E.E., McMahon K.D. Tetracycline resistance genes in activated sludge wastewater treatment plants, Water Research, 41 (2007) 1143-1151.

14. Auvinen H., Havran I., Hubau L., Vanseveren L., Gebhardt W., Linnemann V., Van Oirschot D., Du Laing G., Rousseau D.P.L. Removal of pharmaceuticals by a pilot aerated sub-surface 
flow constructed wetland treating municipal and hospital wastewater, Ecol. Eng., 100 (2017) 157-164.

15. Ávila C., Reyes C., Bayona J.M., García J. Emerging organic contaminant removal depending on primary treatment and operational strategy in horizontal subsurface flow constructed wetlands: Influence of redox, Water Res., 47 (2013) 315-325.

16. Ávila C., Matamoros V., Reyes-Contreras C., Piña B., Casado M., Mita L., Rivetti C., Barata C., García J., Bayona J.M. Attenuation of emerging organic contaminants in a hybrid constructed wetland system under different hydraulic loading rates and their associated toxicological effects in wastewater, Sci. Total Environ., 470-471 (2014) 1272-1280.

17. Ávila C., Bayona J.M., Martín I., Salas J.J., García J. Emerging organic contaminant removal in a full-scale hybrid constructed wetland system for wastewater treatment and reuse, Ecol. Eng., 80 (2015) 108-116.

18. Avisar D., Horovitz I., Lozzi L., Ruggieri F., Baker M., Abel M.-L., Mamane H. Impact of water quality on removal of carbamazepine in natural waters by $\mathrm{N}$-doped $\mathrm{TiO} 2$ photo-catalytic thin film surfaces, J. Hazard. Mater., 244-245 (2013) 463-471.

19. Baker-Austin C., Wright M.S., Stepanauskas R., McArthur J.V. Co-selection of antibiotic and metal resistance, Trends in Microbiology, 14 (2006) 176-182.

20. Banzhaf S., Nödler K., Licha T., Krein A., Scheytt T. Redox-sensitivity and mobility of selected pharmaceutical compounds in a low flow column experiment, Sci. Total Environ., 438 (2012) 113-121.

21. Barber L.B., Leenheer J.A., Noyes T.I., Stiles E.A. Nature and transformation of dissolved organic matter in treatment wetlands, Environ. Sci. Technol., 35 (2001) 4805-4816.

22. Barbieri M., Licha T., Nödler K., Carrera J., Ayora C., Sanchez-Vila X. Fate of $\beta$-blockers in aquifer material under nitrate reducing conditions: Batch experiments, Chemosphere, 89 (2012) $1272-1277$.

23. Barraud O., Baclet M.C., Denis F., Ploy M.C. Quantitative multiplex real-time PCR for detecting class 1, 2 and 3 integrons, Journal of Antimicrobial Chemotherapy, 65 (2010) 16421645.

24. Barron L., Havel J., Purcell M., Szpak M., Kelleher B., Paull B. Predicting sorption of pharmaceuticals and personal care products onto soil and digested sludge using artificial neural networks, Analyst, 134 (2009) 663-670.

25. Bartha B., Huber C., Harpaintner R., Schröder P. Effects of acetaminophen in Brassica juncea $L$. Czern.: investigation of uptake, translocation, detoxification, and the induced defense pathways, Environ. Sci. Pollut. Res., 17 (2010) 1553-1562.

26. Bartha B. Uptake and metabolism of human pharmaceuticals in plants, Ph.D. Dissertation, Technical University of Munich, München, Germany,, (2012).

27. Bartha B., Huber C., Schröder P. Uptake and metabolism of diclofenac in Typha latifoliaHow plants cope with human pharmaceutical pollution, Plant Sci., 227 (2014) 12-20.

28. Batt A.L., Kim S., Aga D.S. Enhanced biodegradation of iopromide and trimethoprim in nitrifying activated sludge, Environ. Sci. Technol., 40 (2006) 7367-7373. 


\section{Bibliography}

29. Baumgarten B., Jährig J., Reemtsma T., Jekel M. Long term laboratory column experiments to simulate bank filtration: Factors controlling removal of sulfamethoxazole, Water Res., 45 (2011) 211-220.

30. Bayo J., Angosto J.M., Gómez-López M.D. Ecotoxicological screening of reclaimed disinfected wastewater by Vibrio fischeri bioassay after a chlorination-dechlorination process, J. Hazard. Mater., 172 (2009) 166-171.

31. Beaumont A.R., Budd M.D. High mortality of the larvae of the common mussel at low concentrations of tributyltin, Mar. Pollut. Bull., 15 (1984) 402-405.

32. Bendz D., Paxéus N.A., Ginn T.R., Loge F.J. Occurrence and fate of pharmaceutically active compounds in the environment, a case study: Höje River in Sweden, J. Hazard. Mater., 122 (2005) 195-204.

33. Benner J., Helbling D.E., Kohler H.-P.E., Wittebol J., Kaiser E., Prasse C., Ternes T.A., Albers C.N., Aamand J., Horemans B., Springael D., Walravens E., Boon N. Is biological treatment a viable alternative for micropollutant removal in drinking water treatment processes?, Water Res., 47 (2013) 5955-5976.

34. Berendonk T.U., Manaia C.M., Merlin C., Fatta-Kassinos D., Cytryn E., Walsh F., Burgmann H., Sorum H., Norstrom M., Pons M.-N., Kreuzinger N., Huovinen P., Stefani S., Schwartz T., Kisand V., Baquero F., Martinez J.L. Tackling antibiotic resistance: the environmental framework, Nature Reviews Microbiology, 13 (2015) 310-317.

35. Berglund B., Khan G.A., Weisner S.E.B., Ehde P.M., Fick J., Lindgren P.-E. Efficient removal of antibiotics in surface-flow constructed wetlands, with no observed impact on antibiotic resistance genes, Sci. Total Environ., 476-477 (2014) 29-37.

36. Bernier S., Surette M. Concentration-dependent activity of antibiotics in natural environments, Front. Microbiol., 4 (2013) 1-14.

37. Bian Z.-Y., Zhu Y.-Q., Zhang J.-X., Ding A.-Z., Wang H. Visible-light driven degradation of ibuprofen using abundant metal-loaded $\mathrm{BiVO}_{4}$ photocatalysts, Chemosphere, 117 (2014) 527531.

38. Birkigt J., Gilevska T., Ricken B., Richnow H.-H., Vione D., Corvini P.F.X., Nijenhuis I., Cichocka D. Carbon stable isotope fractionation of sulfamethoxazole during biodegradation by Microbacterium sp. Strain BR1 and upon direct photolysis, Environ. Sci. Technol., 49 (2015) 6029-6036.

39. Blaise C., Férard J.-F. Small-scale freshwater toxicity investigations: Toxicity test methods, Springer, Dordrecht, 2005.

40. Boleda M.R., Alechaga É., Moyano E., Galceran M.T., Ventura F. Survey of the occurrence of pharmaceuticals in Spanish finished drinking waters, Environ. Sci. Pollut. Res., 21 (2014) 10917-10939.

41. Boreen A.L., Edhlund B.L., Cotner J.B., McNeill K. Indirect photodegradation of dissolved free amino acids: the contribution of singlet oxygen and the differential reactivity of DOM from various sources, Environ. Sci. Technol., 42 (2008) 5492-5498.

42. Bovee T.F.H., Heskamp H.H., Hamers A.R.M., Hoogenboom R.L.A.P., Nielen M.W.F. Validation of a rapid yeast estrogen bioassay, based on the expression of green fluorescent 
protein, for the screening of estrogenic activity in calf urine, Anal. Chim. Acta, 529 (2005) 5764.

43. Brack W. Effect-directed analysis: a promising tool for the identification of organic toxicants in complex mixtures?, Anal. Bioanal. Chem., 377 (2003) 397-407.

44. Bradford M.M. A rapid and sensitive method for the quantitation of microgram quantities of protein utilizing the principle of protein-dye binding, Anal. Biochem., 72 (1976) 248-254.

45. Brausch J.M., Connors K.A., Brooks B.W., Rand G.M. Human pharmaceuticals in the aquatic environment $A$ review of recent toxicological studies and considerations for toxicity testing, in: Rev. Environ. Contam. Toxicol., 2012, pp. 1-99.

46. Breitholtz M., Näslund M., Stråe D., Borg H., Grabic R., Fick J. An evaluation of free water surface wetlands as tertiary sewage water treatment of micro-pollutants, Ecotoxicol. Environ. Saf., 78 (2012) 63-71.

47. Briggs G.G., Bromilow R.H., Evans A.A. Relationships between lipophilicity and root uptake and translocation of non-ionized chemicals by barley, Pestic. Sci., 13 (1982) 495-504.

48. Bui T.X., Choi H. Influence of ionic strength, anions, cations, and natural organic matter on the adsorption of pharmaceuticals to silica, Chemosphere, 80 (2010) 681-686.

49. Calderón-Preciado D., Renault Q., Matamoros V., Cañameras N., Bayona J.M. Uptake of organic emergent contaminants in spath and lettuce: an in vitro experiment, J. Agric. Food. Chem., 60 (2012) 2000-2007.

50. Canonica S., Freiburghaus M. Electron-rich phenols for probing the photochemical reactivity of freshwaters, Environ. Sci. Technol., 35 (2001) 690-695.

51. Canonica S., Laubscher H.U. Inhibitory effect of dissolved organic matter on triplet-induced oxidation of aquatic contaminants, Photoch. Photobio. Sci., 7 (2008) 547-551.

52. Canonica S., Meunier L., Von Gunten U. Phototransformation of selected pharmaceuticals during UV treatment of drinking water, Water Res., 42 (2008) 121-128.

53. Carballa M., Omil F., Lema J.M., Llompart M.a., García-Jares C., Rodríguez I., Gómez M., Ternes T. Behavior of pharmaceuticals, cosmetics and hormones in a sewage treatment plant, Water Res., 38 (2004) 2918-2926.

54. Carmona E., Andreu V., Picó Y. Occurrence of acidic pharmaceuticals and personal care products in Turia River Basin: From waste to drinking water, Sci. Total Environ., 484 (2014) 53-63.

55. Carp O., Huisman C.L., Reller A. Photoinduced reactivity of titanium dioxide, Prog. Solid State Chem., 32 (2004) 33-177.

56. Carr D.L., Morse A.N., Zak J.C., Anderson T.A. Microbially mediated degradation of common pharmaceuticals and personal care products in soil under aerobic and reduced oxygen conditions, Water, Air, Soil Pollut., 216 (2011) 633-642.

57. Carranza-Diaz O., Schultze-Nobre L., Moeder M., Nivala J., Kuschk P., Koeser H. Removal of selected organic micropollutants in planted and unplanted pilot-scale horizontal flow constructed wetlands under conditions of high organic load, Ecol. Eng., 71 (2014) 234-245. 


\section{Bibliography}

58. Carvalho P., Basto M.C., Almeida C.M., Brix H. A review of plant-pharmaceutical interactions: from uptake and effects in crop plants to phytoremediation in constructed wetlands, Environ. Sci. Pollut. Res., 21 (2014) 11729-11763.

59. Carvalho P.N., Basto M.C.P., Almeida C.M.R., Brix H. A review of plant-pharmaceutical interactions: from uptake and effects in crop plants to phytoremediation in constructed wetlands, (2014) 1-35.

60. Celiz M.D., Tso J., Aga D.S. Pharmaceutical metabolites in the environment: Analytical challenges and ecological risks, Environ. Toxicol. Chem., 28 (2009) 2473-2484.

61. Chen B., Hao L., Guo X., Wang N., Ye B. Prevalence of antibiotic resistance genes of wastewater and surface water in livestock farms of Jiangsu Province, China, Environ Sci Pollut Res, (2015) 1-10.

62. Chen B., Liang X., Nie X., Huang X., Zou S., Li X. The role of class I integrons in the dissemination of sulfonamide resistance genes in the Pearl River and Pearl River Estuary, South China, Journal of Hazardous Materials, 282 (2015) 61-67.

63. Chen J., Gu B., LeBoeuf E.J., Pan H., Dai S. Spectroscopic characterization of the structural and functional properties of natural organic matter fractions, Chemosphere, 48 (2002) 59-68.

64. Chen J., Liu Y.S., Su H.C., Ying G.G., Liu F., Liu S.S., He L.Y., Chen Z.F., Yang Y.Q., Chen F.R. Removal of antibiotics and antibiotic resistance genes in rural wastewater by an integrated constructed wetland, Environ. Sci. Pollut. Res., 22 (2015) 1794-1803.

65. Chen J., Wei X.D., Liu Y.S., Ying G.G., Liu S.S., He L.Y., Su H.C., Hu L.X., Chen F.R., Yang Y.Q. Removal of antibiotics and antibiotic resistance genes from domestic sewage by constructed wetlands: Optimization of wetland substrates and hydraulic loading, Sci. Total Environ., 565 (2016) 240-248.

66. Chen T.-C., Yeh K.-J.C., Kuo W.-C., Chao H.-R., Sheu S.-C. Estrogen degradation and sorption onto colloids in a constructed wetland with different hydraulic retention times, J. Hazard. Mater., 277 (2014) 62-68.

67. Chen Y., Hu C., Hu X., Qu J. Indirect photodegradation of amine drugs in aqueous solution under simulated sunlight, Environ. Sci. Technol., 43 (2009) 2760-2765.

68. Chen Y., Zhang K., Zuo Y. Direct and indirect photodegradation of estriol in the presence of humic acid, nitrate and iron complexes in water solutions, Sci. Total Environ., 463-464 (2013) 802-809.

69. Chen Y., Wen Y., Tang Z., Li L., Cai Y., Zhou Q. Removal processes of disinfection byproducts in subsurface-flow constructed wetlands treating secondary effluent, Water Res., 51 (2014) 163-171.

70. Chen Y., Wen Y., Zhou Q., Vymazal J. Effects of plant biomass on nitrogen transformation in subsurface-batch constructed wetlands: A stable isotope and mass balance assessment, Water Res., 63 (2014) 158-167.

71. Chen Y., Wen Y., Zhou Q., Vymazal J. Effects of Plant Biomass on Denitrifying Genes in Subsurface-Flow Constructed Wetlands, Bioresour. Technol., 157 (2014) 341-345. 
72. Chen Y., Wen Y., Tang Z., Huang J., Zhou Q., Vymazal J. Effects of plant biomass on bacterial community structure in constructed wetlands used for tertiary wastewater treatment, Ecol. Eng., 84 (2015) 38-45.

73. Chen Y., Wen Y., Zhou Q., Huang J., Vymazal J., Kuschk P. Sulfate removal and sulfur transformation in constructed wetlands: The roles of filling material and plant biomass, Water Res., 102 (2016) 572-581.

74. Chin Y.-P., Traina S.J., Swank C.R., Backhus D. Abundance and properties of dissolved organic matter in pore waters of a freshwater wetland, Limnol. Oceanogr., 43 (1998) 1287-1296.

75. Christou A., Antoniou C., Christodoulou C., Hapeshi E., Stavrou I., Michael C., Fatta-Kassinos D., Fotopoulos V. Stress-related phenomena and detoxification mechanisms induced by common pharmaceuticals in alfalfa (Medicago sativa L.) plants, Sci. Total Environ., 557-558 (2016) 652-664.

76. Clayton E., Taylor S., Wright B., Wilson I.D. The application of high performance liquid chromatography, coupled to nuclear magnetic resonance spectroscopy and mass spectrometry (HPLC-NMR-MS), to the characterisation of ibuprofen metabolites from human urine, Chromatographia, 47 (1998) 264-270.

77. Coleman H.M., Routledge E.J., Sumpter J.P., Eggins B.R., Byrne J.A. Rapid loss of estrogenicity of steroid estrogens by UVA photolysis and photocatalysis over an immobilised titanium dioxide catalyst, Water Res., 38 (2004) 3233-3240.

78. Coleman J., Blake-Kalff M., Davies E. Detoxification of xenobiotics by plants: chemical modification and vacuolar compartmentation, Trends Plant Sci., 2 (1997) 144-151.

79. Collins C., Fryer M., Grosso A. Plant uptake of non-ionic organic chemicals, Environ. Sci. Technol., 40 (2006) 45-52.

80. Conkle J.L., White J.R., Metcalfe C.D. Reduction of pharmaceutically active compounds by a lagoon wetland wastewater treatment system in Southeast Louisiana, Chemosphere, 73 (2008) 1741-1748.

81. Conkle J.L., Gan J., Anderson M.A. Degradation and sorption of commonly detected PPCPs in wetland sediments under aerobic and anaerobic conditions, Journal of Soils and Sediments, 12 (2012) 1164-1173.

82. Contreras S., Rodriguez M., Al Momani F., Sans C., Esplugas S. Contribution of the ozonation pre-treatment to the biodegradation of aqueous solutions of 2,4-dichlorophenol, Water Res., 37 (2003) 3164-3171.

83. Corseuil H.X., Hunt C.S., Ferreira dos Santos R.C., Alvarez P.J.J. The influence of the gasoline oxygenate ethanol on aerobic and anaerobic BTX biodegradation, Water Res., 32 (1998) 20652072.

84. Cui H., Hense B.A., Müller J., Schröder P. Short term uptake and transport process for metformin in roots of Phragmites australis and Typha latifolia, Chemosphere, 134 (2015) 307312.

85. Czajka C.P., Londry K.L. Anaerobic biotransformation of estrogens, Sci. Total Environ., 367 (2006) 932-941. 


\section{Bibliography}

86. Dalrymple O.K., Yeh D.H., Trotz M.A. Removing pharmaceuticals and endocrine-disrupting compounds from wastewater by photocatalysis, J. Chem. Technol. Biotechnol., 82 (2007) 121134.

87. Dan A., Yang Y., Dai Y.-n., Chen C.-x., Wang S.-y., Tao R. Removal and factors influencing removal of sulfonamides and trimethoprim from domestic sewage in constructed wetlands, Bioresour. Technol., 146 (2013) 363-370.

88. David A., Pancharatna K. Developmental anomalies induced by a non-selective COX inhibitor (ibuprofen) in zebrafish (Danio rerio), Environ. Toxicol. Pharmacol., 27 (2009) 390-395.

89. Dawas-Massalha A., Gur-Reznik S., Lerman S., Sabbah I., Dosoretz C.G. Co-metabolic oxidation of pharmaceutical compounds by a nitrifying bacterial enrichment, Bioresour. Technol., 167 (2014) 336-342.

90. De Gusseme B., Pycke B., Hennebel T., Marcoen A., Vlaeminck S.E., Noppe H., Boon N., Verstraete W. Biological removal of $17 \alpha$-ethinylestradiol by a nitrifier enrichment culture in a membrane bioreactor, Water Res., 43 (2009) 2493-2503.

91. De la Cruz N., Dantas R.F., Gimenez J., Esplugas S. Photolysis and $\mathrm{TiO}_{2}$ photocatalysis of the pharmaceutical propranolol: Solar and artificial light, Appl. Catal,. B, 130 (2013) 249-256.

92. de Wilt A., Butkovskyi A., Tuantet K., Leal L.H., Fernandes T.V., Langenhoff A., Zeeman G. Micropollutant removal in an algal treatment system fed with source separated wastewater streams, J. Hazard. Mater., 304 (2016) 84-92.

93. Dennison W.C., Orth R.J., Moore K.A., Stevenson J.C., Carter V., Kollar S., Bergstrom P.W., Batiuk R.A. Assessing Water Quality with Submersed Aquatic Vegetation, Bioscience, 43 (1993) 86-94.

94. Di Nica V., Villa S., Finizio A. Toxicity of individual pharmaceuticals and their mixtures to Aliivibrio fischeri: Experimental results for single compounds and considerations of their mechanisms of action and potential acute effects on aquatic organisms, Environ. Toxicol. Chem., 36 (2017) 807-814.

95. Diniz M.S., Salgado R., Pereira V.J., Carvalho G., Oehmen A., Reis M.A.M., Noronha J.P. Ecotoxicity of ketoprofen, diclofenac, atenolol and their photolysis byproducts in zebrafish (Danio rerio), Sci. Total Environ., 505 (2015) 282-289.

96. Directive C. 91/271/EEC of 21 May 1991 concerning urban waste treatment, Off J Eur Communities, L, 135 (1991).

97. Dobor J., Varga M., Záray G. Biofilm controlled sorption of selected acidic drugs on river sediments characterized by different organic carbon content, Chemosphere, 87 (2012) 105-110.

98. Doll T.E., Frimmel F.H. Kinetic study of photocatalytic degradation of carbamazepine, clofibric acid, iomeprol and iopromide assisted by different $\mathrm{TiO} 2$ materials - determination of intermediates and reaction pathways, Water Res., 38 (2004) 955-964.

99. Dong M.M., Trenholm R., Rosario-Ortiz F.L. Photochemical degradation of atenolol, carbamazepine, meprobamate, phenytoin and primidone in wastewater effluents, J. Hazard. Mater., (2004).

100. Dordio A., Palace A., Pinto A.P. Wetlands: Water Living Filters?, in: R.E. Russo (Ed.), Nova Science Publishers, 2008. 
101. Dordio A., Carvalho A.J.P., Teixeira D.M., Dias C.B., Pinto A.P. Removal of pharmaceuticals in microcosm constructed wetlands using Typha spp. and LECA, Bioresour. Technol., 101 (2010) 886-892.

102. Dordio A., Ferro R., Teixeira D., Palace A.J., Pinto A.P., Dias C.M.B. Study on the use of Typha spp. for the phytotreatment of water contaminated with ibuprofen, Int. J. Environ. Anal. Chem., 91 (2011) 654-667.

103. Dordio A.V., Duarte C., Barreiros M., Carvalho A.J., Pinto A.P., da Costa C.T. Toxicity and removal efficiency of pharmaceutical metabolite clofibric acid by Typha spp.-potential use for phytoremediation?, Bioresour. Technol., 100 (2009) 1156-1161.

104. Dordio A.V., Belo M., Martins Teixeira D., Palace Carvalho A.J., Dias C.M., Pico Y., Pinto A.P. Evaluation of carbamazepine uptake and metabolization by Typha spp., a plant with potential use in phytotreatment, Bioresour. Technol., 102 (2011) 7827-7834.

105. Dordio A.V., Carvalho A.J. Organic xenobiotics removal in constructed wetlands, with emphasis on the importance of the support matrix, J. Hazard. Mater., 252-253 (2013) 272-292.

106. Drillia P., Stamatelatou K., Lyberatos G. Fate and mobility of pharmaceuticals in solid matrices, Chemosphere, 60 (2005) 1034-1044.

107. EEC Council Directive 67/548/EEC of 27 June 1967 on the approximation of laws, regulations and administrative provisions relating to the classification, packaging and labelling of dangerous substances, Off. J. Eur. Commun., 276 (1967) 1-98.

108. Eger P. Wetland treatment for trace metal removal from mine drainage: The importance of aerobic and anaerobic processes, Water Sci. Technol., 29 (1994) 249-256.

109. Eisentraeger A., Dott W., Klein J., Hahn S. Comparative studies on algal toxicity testing using fluorometric microplate and Erlenmeyer flask growth-inhibition assays, Ecotoxicol. Environ. Saf., 54 (2003) 346-354.

110. Elsner M., Zwank L., Hunkeler D., Schwarzenbach R.P. A new concept linking observable stable isotope fractionation to transformation pathways of organic pollutants, Environ. Sci. Technol., 39 (2005) 6896-6916.

111. Enne V.I., Livermore D.M., Stephens P., Hall L.M.C. Persistence of sulphonamide resistance in Escherichia coli in the UK despite national prescribing restriction, The Lancet, 357 (2001) $1325-1328$

112. Evans P.J., Mang D.T., Young L.Y. Degradation of toluene and m-xylene and transformation of o-xylene by denitrifying enrichment cultures, Appl. Environ. Microbiol., 57 (1991) 450-454.

113. Fai P.B., Grant A. A rapid resazurin bioassay for assessing the toxicity of fungicides, Chemosphere, 74 (2009) 1165-1170.

114. Falås P., Andersen H.R., Ledin A., La Cour Jansen J. Impact of solid retention time and nitrification capacity on the ability of activated sludge to remove pharmaceuticals, Environ. Technol., 33 (2012) 865-872.

115. Falås P., Wick A., Castronovo S., Habermacher J., Ternes T.A., Joss A. Tracing the limits of organic micropollutant removal in biological wastewater treatment, Water Res., 95 (2016) 240249. 


\section{Bibliography}

116. Fan J., Wang W., Zhang B., Guo Y., Ngo H.H., Guo W., Zhang J., Wu H. Nitrogen removal in intermittently aerated vertical flow constructed wetlands: Impact of influent $\mathrm{COD} / \mathrm{N}$ ratios, Bioresour. Technol., 143 (2013) 461-466.

117. Fang H., Zhang Q., Nie X., Chen B., Xiao Y., Zhou Q., Liao W., Liang X. Occurrence and elimination of antibiotic resistance genes in a long-term operation integrated surface flow constructed wetland, Chemosphere, 173 (2017) 99-106.

118. Fatta-Kassinos D., Meric S., Nikolaou A. Pharmaceutical residues in environmental waters and wastewater: current state of knowledge and future research, Anal. Bioanal. Chem., 399 (2011) 251-275.

119. Faulwetter J.L., Gagnon V., Sundberg C., Chazarenc F., Burr M.D., Brisson J., Camper A.K., Stein O.R. Microbial processes influencing performance of treatment wetlands: A review, Ecol. Eng., 35 (2009) 987-1004.

120. Fent K., Weston A.A., Caminada D. Ecotoxicology of human pharmaceuticals, Aquat. Toxicol., 76 (2006) 122-159.

121. Fernandez-Fontaina E., Gomes I.B., Aga D.S., Omil F., Lema J.M., Carballa M.

Biotransformation of pharmaceuticals under nitrification, nitratation and heterotrophic conditions, Sci. Total Environ., 541 (2016) 1439-1447.

122. Fernández-Ibáñez P., Blanco J., Malato S., Nieves F.J.d.l. Application of the colloidal stability of $\mathrm{TiO}_{2}$ particles for recovery and reuse in solar photocatalysis, Water Res., 37 (2003) 31803188.

123. Fernández A., Lassaletta G., Jiménez V.M., Justo A., González-Elipe A.R., Herrmann J.M., Tahiri H., Ait-Ichou Y. Preparation and characterization of $\mathrm{TiO}_{2}$ photocatalysts supported on various rigid supports (glass, quartz and stainless steel). Comparative studies of photocatalytic activity in water purification, Appl. Catal,. B, 7 (1995) 49-63.

124. Ferrando-Climent L., Collado N., Buttiglieri G., Gros M., Rodriguez-Roda I., RodriguezMozaz S., Barceló D. Comprehensive study of ibuprofen and its metabolites in activated sludge batch experiments and aquatic environment, Sci. Total Environ., 438 (2012) 404-413.

125. Field J.A., Cervantes F.J., van der Zee F.P., Lettinga G. Role of quinones in the biodegradation of priority pollutants: a review, Water Sci. Technol., 42 (2000) 215-222.

126. Fierer N., Jackson J.A., Vilgalys R., Jackson R.B. Assessment of soil microbial community structure by use of taxon-specific quantitative PCR assays, Appl. Environ. Microbiol., 71 (2005) 4117-4120.

127. Focazio M.J., Kolpin D.W., Barnes K.K., Furlong E.T., Meyer M.T., Zaugg S.D., Barber L.B., Thurman M.E. A national reconnaissance for pharmaceuticals and other organic wastewater contaminants in the United States - II) Untreated drinking water sources, Sci. Total Environ., 402 (2008) 201-216.

128. Foekema E. De invloed van moerassystemen op de milieukwaliteit van rwzi effluent en aanbevelingen tot optimalisering. IMARES rapport C005/12, in, 2012.

129. Foght J., April T., Biggar K., Aislabie J. Bioremediation of DDT-contaminated soils: a review, Biorem. J., 5 (2001) 225-246. 
130. Forrez I., Carballa M., Boon N., Verstraete W. Biological removal of 17 $\alpha$-ethinylestradiol (EE2) in an aerated nitrifying fixed bed reactor during ammonium starvation, J. Chem. Technol.

Biotechnol., 84 (2009) 119-125.

131. Freitas R., Almeida Â., Pires A., Velez C., Calisto V., Schneider R.J., Esteves V.I., Wrona F.J., Figueira E., Soares A.M.V.M. The effects of carbamazepine on macroinvertebrate species: comparing bivalves and polychaetes biochemical responses, Water Res., 85 (2015) 137-147.

132. Froehner S., Piccioni W., Machado K.S., Aisse M.M. Removal capacity of caffeine, hormones, and bisphenol by aerobic and anaerobic sewage treatment, Water, Air, Soil Pollut., 216 (2011) 463-471.

133. Furlong E.T., Batt A.L., Glassmeyer S.T., Noriega M.C., Kolpin D.W., Mash H., Schenck K.M. Nationwide reconnaissance of contaminants of emerging concern in source and treated drinking waters of the United States: Pharmaceuticals, Sci. Total Environ., 579 (2017) 1629-1642.

134. Gao J., O'Brien J., Du P., Li X., Ort C., Mueller J.F., Thai P.K. Measuring selected PPCPs in wastewater to estimate the population in different cities in China, Sci. Total Environ., 568 (2016) $164-170$.

135. Gao R.Q., Hou X.M. Preparation and photo-catalytic activity of $\mathrm{TiO}_{2}$-coated medical stonebased porous ceramics, Int. J. Min. Met. Mater., 20 (2013) 593-597.

136. García-Galán M.J., Díaz-Cruz M.S., Barceló D. Kinetic studies and characterization of photolytic products of sulfamethazine, sulfapyridine and their acetylated metabolites in water under simulated solar irradiation, Water Res., 46 (2012) 711-722.

137. Garcia-Rodríguez A., Matamoros V., Fontàs C., Salvadó V. The ability of biologically based wastewater treatment systems to remove emerging organic contaminants-a review, Environ. Sci. Pollut. Res., 21 (2014) 11708-11728.

138. Gaya U.I., Abdullah A.H. Heterogeneous photocatalytic degradation of organic contaminants over titanium dioxide: A review of fundamentals, progress and problems, Journal of Photochemistry and Photobiology C: Photochemistry Reviews, 9 (2008) 1-12.

139. Gerhardt K.E., Huang X.-D., Glick B.R., Greenberg B.M. Phytoremediation and rhizoremediation of organic soil contaminants: Potential and challenges, Plant Sci., 176 (2009) 20-30.

140. Gersberg R.M., Elkins B.V., Lyon S.R., Goldman C.R. Role of aquatic plants in wastewater treatment by artificial wetlands, Water Res., 20 (1986) 363-368.

141. Gillings M.R., Gaze W.H., Pruden A., Smalla K., Tiedje J.M., Zhu Y.-G. Using the class 1 integron-integrase gene as a proxy for anthropogenic pollution, 9 (2015) 1269-1279.

142. Giri R.R., Ozaki H., Ota S., Takanami R., Taniguchi S. Degradation of common pharmaceuticals and personal care products in mixed solutions by advanced oxidation techniques, Int. J. Environ. Sci. Technol. (Tehran), 7 (2010) 251-260.

143. Gros M., Petrović M., Barceló D. Multi-residue analytical methods using LC-tandem MS for the determination of pharmaceuticals in environmental and wastewater samples: a review, Anal. Bioanal. Chem., 386 (2006) 941-952. 


\section{Bibliography}

144. Gros M., Petrovic M., Ginebreda A., Barcelo D. Removal of pharmaceuticals during wastewater treatment and environmental risk assessment using hazard indexes, Environ. Int., 36 (2010) 15-26.

145. Gros M., Petrović M., Ginebreda A., Barceló D. Removal of pharmaceuticals during wastewater treatment and environmental risk assessment using hazard indexes, Environ. Int., 36 (2010) 15-26.

146. Grossberger A., Hadar Y., Borch T., Chefetz B. Biodegradability of pharmaceutical compounds in agricultural soils irrigated with treated wastewater, Environ. Pollut., 185 (2014) 168-177.

147. Guerard J., Miller P., Trouts T., Chin Y.-P. The role of fulvic acid composition in the photosensitized degradation of aquatic contaminants, Aquat. Sci., 71 (2009) 160-169.

148. Guillard R.R.L., Lorenzen C.J. Yellow-green algae with chlorophyllide C, J. Phycol., 8 (1972) $10-14$.

149. Gullberg E., Cao S., Berg O.G., Ilbäck C., Sandegren L., Hughes D., Andersson D.I. Selection of resistant bacteria at very low antibiotic concentrations, PLoS Path., 7 (2011) e1002158.

150. Gunasekaran S., Sankari G., Ponnusamy S. Vibrational spectral investigation on xanthine and its derivatives - theophylline, caffeine and theobromine, Spectrochimica Acta Part A: Molecular and Biomolecular Spectroscopy, 61 (2005) 117-127.

151. Hai F.I., Li X., Price W.E., Nghiem L.D. Removal of carbamazepine and sulfamethoxazole by MBR under anoxic and aerobic conditions, Bioresour. Technol., 102 (2011) 10386-10390.

152. Hamers T., Molin K.R.J., Koeman J.H., Murk A.J. A small-volume bioassay for quantification of the esterase inhibiting potency of mixtures of organophosphate and carbamate insecticides in rainwater: Development and optimization, Toxicol. Sci., 58 (2000) 60-67.

153. Hamers T., Smit M.G.D., Murk A.J., Koeman J.H. Biological and chemical analysis of the toxic potency of pesticides in rainwater, Chemosphere, 45 (2001) 609-624.

154. Hamers T., van den Brink P.J., Mos L., van der Linden S.C., Legler J., Koeman J.H., Murk A.J. Estrogenic and esterase-inhibiting potency in rainwater in relation to pesticide concentrations, sampling season and location, Environ. Pollut., 123 (2003) 47-65.

155. He Y., Chen W., Zheng X., Wang X., Huang X. Fate and removal of typical pharmaceuticals and personal care products by three different treatment processes, Sci. Total Environ., 447 (2013) 248-254.

156. He Y., Sutton N.B., Rijnaarts H.H.M., Langenhoff A.A.M. Degradation of pharmaceuticals in wastewater using immobilized $\mathrm{TiO}_{2}$ photocatalysis under simulated solar irradiation, Appl. Catal,. B, 182 (2016) 132-141.

157. He Y., Langenhoff A.A.M., Comans R.N.J., Sutton N.B., Rijnaarts H.H.M. Effects of dissolved organic matter and nitrification on biodegradation of pharmaceuticals in aerobic enrichment cultures, In preparation, (2017).

158. He Y., Langenhoff A.A.M., Sutton N.B., Rijnaarts H.H.M., Blokland M.H., Chen F., Huber C., Schröder P. Metabolism of Ibuprofen by Phragmites australis: Uptake and Phytodegradation, Environ. Sci. Technol., (2017). 
159. He Y., Nurul S., Schmitt H., Sutton N.B., Murk T.A.J., Blokland M.H., Rijnaarts H.H.M., Langenhoff A.A.M. Evaluation of attenuation of pharmaceuticals, toxicity and antibiotic resistance genes in constructed wetlands treating wastewater effluents, In preparation, (2017).

160. Headley T.R., Kadlec R.H. Conducting hydraulic tracer studies of constructed wetlands: a practical guide, Ecohydrology \& Hydrobiology, 7 (2007) 269-282.

161. Heberer T., Massmann G., Fanck B., Taute T., Dünnbier U. Behaviour and redox sensitivity of antimicrobial residues during bank filtration, Chemosphere, 73 (2008) 451-460.

162. Heijman S.G.J., Verliefde A.R.D., Cornelissen E.R., Amy G., van Dijk J.C. Influence of natural organic matter (NOM) fouling on the removal of pharmaceuticals by nanofiltration and activated carbon filtration, Water Science and Technology: Water Supply, 7 (2007) 17-23.

163. Helal H.M., Sauerbeck D. Carbon Turnover in the Rhizosphere, Zeitschrift für Pflanzenernährung und Bodenkunde, 152 (1989) 211-216.

164. Henschel K.P., Wenzel A., Diedrich M., Fliedner A. Environmental hazard assessment of pharmaceuticals, Regul. Toxicol. Pharm., 25 (1997) 220-225.

165. Hernando M.D., Mezcua M., Fernández-Alba A.R., Barceló D. Environmental risk assessment of pharmaceutical residues in wastewater effluents, surface waters and sediments, Talanta, 69 (2006) 334-342.

166. Heuer H., Smalla K. Manure and sulfadiazine synergistically increased bacterial antibiotic resistance in soil over at least two months, Environ. Microbiol., 9 (2007) 657-666.

167. Heuer H., Focks A., Lamshöft M., Smalla K., Matthies M., Spiteller M. Fate of sulfadiazine administered to pigs and its quantitative effect on the dynamics of bacterial resistance genes in manure and manured soil, Soil Biology and Biochemistry, 40 (2008) 1892-1900.

168. Hijosa-Valsero M., Matamoros V., Martín-Villacorta J., Bécares E., Bayona J.M. Assessment of full-scale natural systems for the removal of PPCPs from wastewater in small communities, Water Res., 44 (2010) 1429-1439.

169. Hijosa-Valsero M., Matamoros V., Sidrach-Cardona R., Martin-Villacorta J., Becares E., Bayona J.M. Comprehensive assessment of the design configuration of constructed wetlands for the removal of pharmaceuticals and personal care products from urban wastewaters, Water Res., 44 (2010) 3669-3678.

170. Hijosa-Valsero M., Matamoros V., Sidrach-Cardona R., Pedescoll A., Martín-Villacorta J., García J., Bayona J.M., Bécares E. Influence of design, physico-chemical and environmental parameters on pharmaceuticals and fragrances removal by constructed wetlands, Water Sci. Technol., 63 (2011) 2527-2534.

171. Hijosa-Valsero M., Reyes-Contreras C., Domínguez C., Bécares E., Bayona J.M. Behaviour of pharmaceuticals and personal care products in constructed wetland compartments: Influent, effluent, pore water, substrate and plant roots, Chemosphere, 145 (2016) 508-517.

172. Hofstetter T.B., Berg M. Assessing transformation processes of organic contaminants by compound-specific stable isotope analysis, TrAC, Trends Anal. Chem., 30 (2011) 618-627.

173. Hollender J., Zimmermann S.G., Koepke S., Krauss M., McArdell C.S., Ort C., Singer H., von Gunten U., Siegrist H. Elimination of organic micropollutants in a municipal wastewater 


\section{Bibliography}

treatment plant upgraded with a full-scale post-ozonation followed by sand filtration, Environ. Sci. Technol., 43 (2009) 7862-7869.

174. Holliger C., Schraa G., Stams A.J.M., Zehnder A.J.B. A highly purified enrichment culture couples the reductive dechlorination of tetrachloroethene to growth, Appl. Environ. Microbiol., 59 (1993) 2991-2997.

175. Holst S.v. A preliminary study of advanced treatment technologies for removing antibiotics and antibiotic resistance genes from wastewater, in: Sub-department of Environmental Technology, Wageningen University, The Netherlands, 2017.

176. Hu Y., Zhao Y., Zhao X., Kumar J.L.G. High rate nitrogen removal in an alum sludge-based intermittent aeration constructed wetland, Environ. Sci. Technol., 46 (2012) 4583-4590.

177. Huang X., Liu C., Li K., Su J., Zhu G., Liu L. Performance of vertical up-flow constructed wetlands on swine wastewater containing tetracyclines and tet genes, Water Res., 70 (2015) 109-117.

178. Huggett D.B., Brooks B.W., Peterson B., Foran C.M., Schlenk D. Toxicity of select beta adrenergic receptor-blocking pharmaceuticals ( $\beta$-blockers) on aquatic organisms, Arch. Environ. Contam. Toxicol., 43 (2002) 229-235.

179. Hund-Rinke K., Simon M. Ecotoxic effect of photocatalytic active nanoparticles $\left(\mathrm{TiO}_{2}\right)$ on algae and daphnids (8 pp), Environ. Sci. Pollut. Res., 13 (2006) 225-232.

180. Hussain S.A., Prasher S.O., Patel R.M. Removal of ionophoric antibiotics in free water surface constructed wetlands, Ecol. Eng., 41 (2012) 13-21.

181. Iasur-Kruh L., Hadar Y., Minz D. Isolation and bioaugmentation of an estradiol-degrading bacterium and its integration into a mature biofilm, Appl. Environ. Microbiol., 77 (2011) 37343740 .

182. Illés E., Takács E., Dombi A., Gajda-Schrantz K., Rácz G., Gonter K., Wojnárovits L. Hydroxyl radical induced degradation of ibuprofen, Sci. Total Environ., 447 (2013) 286-292.

183. Im J., Löffler F.E. Fate of bisphenol A in terrestrial and aquatic environments, Environ. Sci. Technol., 50 (2016) 8403-8416.

184. Jacobs L.E., Fimmen R.L., Chin Y.-P., Mash H.E., Weavers L.K. Fulvic acid mediated photolysis of ibuprofen in water, Water Res., 45 (2011) 4449-4458.

185. Jasper J.T., Sedlak D.L. Phototransformation of wastewater-derived Trace organic contaminants in open-water unit process treatment wetlands, Environ. Sci. Technol., 47 (2013) 10781-10790.

186. Ji Y., Zeng C., Ferronato C., Chovelon J.-M., Yang X. Nitrate-induced photodegradation of atenolol in aqueous solution: Kinetics, toxicity and degradation pathways, Chemosphere, 88 (2012) 644-649.

187. Jiang J.-Q., Zhou Z., Sharma V.K. Occurrence, transportation, monitoring and treatment of emerging micro-pollutants in waste water - A review from global views, Microchem. J., 110 (2013) 292-300.

188. Jong P.d., Evenblij H. Verkenningen zuiveringstechnieken en KRW, in, STOWA, Utrecht, The Netherlands, 2005. 
189. Jonker M.T.O., Brils J.M., Sinke A.J.C., Murk A.J., Koelmans A.A. Weathering and toxicity of marine sediments contaminated with oils and polycyclic aromatic hydrocarbons, Environ.

Toxicol. Chem., 25 (2006) 1345-1353.

190. Joss A., Zabczynski S., Göbel A., Hoffmann B., Löffler D., McArdell C.S., Ternes T.A., Thomsen A., Siegrist H. Biological degradation of pharmaceuticals in municipal wastewater treatment: Proposing a classification scheme, Water Res., 40 (2006) 1686-1696.

191. Kadlec R., Wallace S. Introduction to Treatment Wetlands, in: Treatment Wetlands, CRC press, Boca Raton, Florida 2009, pp. 3-19.

192. Karnjanapiboonwong A., Suski J.G., Shah A.A., Cai Q., Morse A.N., Anderson T.A. Occurrence of PPCPs at a wastewater treatment plant and in soil and groundwater at a land application site, Water, Air, Soil Pollut., 216 (2010) 257-273.

193. Keane D., Basha S., Nolan K., Morrissey A., Oelgemöller M., Tobin J. Photodegradation of famotidine by integrated photocatalytic adsorbent (IPCA) and kinetic study, Catal. Lett., 141 (2011) 300-308.

194. Kemper N. Veterinary antibiotics in the aquatic and terrestrial environment, Ecol. Indicators, 8 (2008) 1-13.

195. Khadka B., Munir T.M., Strack M. Dissolved organic carbon in a constructed and natural fens in the Athabasca oil sands region, Alberta, Canada, Sci. Total Environ., 557-558 (2016) 579589.

196. Khunjar W.O., Mackintosh S.A., Skotnicka-Pitak J., Baik S., Aga D.S., Love N.G. Elucidating the relative roles of ammonia oxidizing and heterotrophic bacteria during the biotransformation of 17a-ethinylestradiol and trimethoprim, Environ. Sci. Technol., 45 (2011) 3605-3612.

197. Kim H.-C., Noh J.H., Chae S.-R., Choi J., Lee Y., Maeng S.K. A multi-parametric approach assessing microbial viability and organic matter characteristics during managed aquifer recharge, Sci. Total Environ., 524-525 (2015) 290-299.

198. Kim Y.-M., Jeon J.-R., Murugesan K., Kim E.-J., Chang Y.-S. Biodegradation of 1,4-dioxane and transformation of related cyclic compounds by a newly isolated Mycobacterium sp. PH-06, Biodegradation, 20 (2008) 511.

199. Kime D.E., Nash J.P. Gamete viability as an indicator of reproductive endocrine disruption in fish, Sci. Total Environ., 233 (1999) 123-129.

200. Kivaisi A.K. The potential for constructed wetlands for wastewater treatment and reuse in developing countries: a review, Ecol. Eng., 16 (2001) 545-560.

201. Klamerth N., Rizzo L., Malato S., Maldonado M.I., Agüera A., Fernández-Alba A.R. Degradation of fifteen emerging contaminants at $\mu \mathrm{g} \mathrm{L}-1$ initial concentrations by mild solar photo-Fenton in MWTP effluents, Water Res., 44 (2010) 545-554.

202. Klein R.M. Failure of supplementary ultraviolet radiation to enhance flower color under greenhouse conditions, HortScience, 25 (1990) 307-308.

203. Knapp C.W., Dolfing J., Ehlert P.A.I., Graham D.W. Evidence of Increasing Antibiotic Resistance Gene Abundances in Archived Soils since 1940, Environ. Sci. Technol., 44 (2010) 580-587. 


\section{Bibliography}

204. Koba O., Golovko O., Kodešová R., Klement A., Grabic R. Transformation of atenolol, metoprolol, and carbamazepine in soils: The identification, quantification, and stability of the transformation products and further implications for the environment, Environ. Pollut., 218 (2016) $574-585$

205. Konstantinou I.K., Albanis T.A. $\mathrm{TiO}_{2}$-assisted photocatalytic degradation of azo dyes in aqueous solution: kinetic and mechanistic investigations: A review, Appl. Catal,. B, 49 (2004) $1-14$.

206. Kotyza J., Soudek P., Kafka Z., Vaněk T. Phytoremediation of pharmaceuticals—preliminary study, Int. J. Phytorem., 12 (2010) 306-316.

207. Kramer C., Gleixner G. Soil organic matter in soil depth profiles: Distinct carbon preferences of microbial groups during carbon transformation, Soil Biol. Biochem., 40 (2008) 425-433.

208. Kummerer K. Pharmaceuticals in the Environment, Annu. Rev. Environ. Resour., 35 (2010) $57-$ 75.

209. Kunkel U., Radke M. Fate of pharmaceuticals in rivers: Deriving a benchmark dataset at favorable attenuation conditions, Water Res., 46 (2012) 5551-5565.

210. Langenhoff A., Inderfurth N., Veuskens T., Schraa G., Blokland M., Kujawa-Roeleveld K., Rijnaarts H. Microbial removal of the pharmaceutical compounds ibuprofen and diclofenac from wastewater, Biomed Research International, (2013).

211. Langenhoff A.A.M., Zehnder A.J.B., Schraa G. Behaviour of toluene, benzene and naphthalene under anaerobic conditions in sediment columns, Biodegradation, 7 (1996) 267-274.

212. Langenhoff A.A.M. Biotransformation of toluene, benzene and naphthalene under anaerobic conditions, in: Microbiology Department, Wageningen Agricultural University, The Netherlands, 1997.

213. Langenhoff A.A.M., Brouwers-Ceiler D.L., Engelberting J.H.L., Quist J.J., Wolkenfelt J.G.P.N., Zehnder A.J.B., Schraa G. Microbial reduction of manganese coupled to toluene oxidation, FEMS Microbiol. Ecol., 22 (1997) 119-127.

214. LaPara T.M., Madson M., Borchardt S., Lang K.S., Johnson T.J. Multiple discharges of treated municipal wastewater have a small effect on the quantities of numerous antibiotic resistance determinants in the upper Mississippi River, Environ. Sci. Technol., 49 (2015) 11509-11515.

215. Leal J.F., Esteves V.I., Santos E.B.H. Does light-screening by humic substances completely explain their retardation effect on contaminants photo-degradation?, J. Environ. Chem. Eng., (2015).

216. Lee E., Shon H.K., Cho J. Role of wetland organic matters as photosensitizer for degradation of micropollutants and metabolites, J. Hazard. Mater., 276 (2014) 1-9.

217. Lee S., Kang S.-I., Lim J.-L., Huh Y.J., Kim K.-S., Cho J. Evaluating controllability of pharmaceuticals and metabolites in biologically engineered processes, using corresponding octanol-water distribution coefficient, Ecol. Eng., 37 (2011) 1595-1600.

218. Legler J., Jonas A., Lahr J., Vethaak A.D., Brouwer A., Murk A.J. Biological measurement of estrogenic activity in urine and bile conjugates with the in vitro ER-CALUX reporter gene assay, Environ. Toxicol. Chem., 21 (2002) 473-479. 
219. Lerch T.Z., Dignac M.-F., Nunan N., Bardoux G., Barriuso E., Mariotti A. Dynamics of soil microbial populations involved in 2,4-D biodegradation revealed by FAME-based Stable Isotope Probing, Soil Biol. Biochem., 41 (2009) 77-85.

220. Li F., Jiang B., Nastold P., Kolvenbach B.A., Chen J., Wang L., Guo H., Corvini P.F.-X., Ji R. Enhanced transformation of tetrabromobisphenol A by nitrifiers in nitrifying activated sludge, Environ. Sci. Technol., 49 (2015) 4283-4292.

221. Li J., Dodgen L., Ye Q., Gan J. Degradation kinetics and metabolites of carbamazepine in soil, Environ. Sci. Technol., 47 (2013) 3678-3684.

222. Li W.C. Occurrence, sources, and fate of pharmaceuticals in aquatic environment and soil, Environ. Pollut., 187 (2014) 193-201.

223. Li X., Zheng W., Kelly W.R. Occurrence and removal of pharmaceutical and hormone contaminants in rural wastewater treatment lagoons, Sci. Total Environ., 445-446 (2013) 22-28.

224. Li Y., Zhu G., Ng W.J., Tan S.K. A review on removing pharmaceutical contaminants from wastewater by constructed wetlands: Design, performance and mechanism, Sci. Total Environ., 468-469 (2014) 908-932.

225. Li Y., Wu B., Zhu G., Liu Y., Ng W.J., Appan A., Tan S.K. High-throughput pyrosequencing analysis of bacteria relevant to cometabolic and metabolic degradation of ibuprofen in horizontal subsurface flow constructed wetlands, Sci. Total Environ., 562 (2016) 604-613.

226. Li Y., Zhang J., Zhu G., Liu Y., Wu B., Ng W.J., Appan A., Tan S.K. Phytoextraction, phytotransformation and rhizodegradation of ibuprofen associated with Typha angustifolia in a horizontal subsurface flow constructed wetland, Water Res., 102 (2016) 294-304.

227. Li Z., Randak T. Residual pharmaceutically active compounds (PhACs) in aquatic environment-status, toxicity and kinetics: a review, Vet. Med. (Praha), 52 (2009) 295-314.

228. Li Z., Sobek A., Radke M. Flume experiments to investigate the environmental fate of pharmaceuticals and their transformation products in streams, Environ. Sci. Technol., 49 (2015) 6009-6017.

229. Lim M.-H., Snyder S.A., Sedlak D.L. Use of biodegradable dissolved organic carbon (BDOC) to assess the potential for transformation of wastewater-derived contaminants in surface waters, Water Res., 42 (2008) 2943-2952.

230. Lin A.Y.-C., Lin C.-A., Tung H.-H., Chary N.S. Potential for biodegradation and sorption of acetaminophen, caffeine, propranolol and acebutolol in lab-scale aqueous environments, J. Hazard. Mater., 183 (2010) 242-250.

231. Lin K., Gan J. Sorption and degradation of wastewater-associated non-steroidal antiinflammatory drugs and antibiotics in soils, Chemosphere, 83 (2011) 240-246.

232. Lindeboom R.E.F., Fermoso F.G., Weijma J., Zagt K., van Lier J.B. Autogenerative high pressure digestion: anaerobic digestion and biogas upgrading in a single step reactor system, Water Sci. Technol., 64 (2011) 647-653.

233. Liqiang J., Yichun Q., Baiqi W., Shudan L., Baojiang J., Libin Y., Wei F., Honggang F., Jiazhong S. Review of photoluminescence performance of nano-sized semiconductor materials and its relationships with photocatalytic activity, Sol. Energy Mater. Sol. Cells, 90 (2006) 17731787. 


\section{Bibliography}

234. Liu D., Ge Y., Chang J., Peng C., Gu B., Chan G.Y.S., Wu X. Constructed wetlands in China: recent developments and future challenges, Front. Ecol. Environ., 7 (2009) 261-268.

235. Liu J., Wong M. Pharmaceuticals and personal care products (PPCPs): A review on environmental contamination in China, Environ. Int., 59 (2013) 208-224.

236. Liu L., Liu C., Zheng J., Huang X., Wang Z., Liu Y., Zhu G. Elimination of veterinary antibiotics and antibiotic resistance genes from swine wastewater in the vertical flow constructed wetlands, Chemosphere, 91 (2013) 1088-1093.

237. Liu Q.-T., Williams H.E. Kinetics and degradation products for direct photolysis of $\beta$-blockers in water, Environ. Sci. Technol., 41 (2007) 803-810.

238. Liu W., Sutton N.B., Rijnaarts H.H.M., Langenhoff A.A.M. Pharmaceutical removal from water with iron- or manganese-based technologies: A review, Crit. Rev. Environ. Sci. Technol., 46 (2016) 1584-1621.

239. Llorens E., Matamoros V., Domingo V., Bayona J.M., García J. Water quality improvement in a full-scale tertiary constructed wetland: Effects on conventional and specific organic contaminants, Sci. Total Environ., 407 (2009) 2517-2524.

240. Löffler D., Ternes T.A. Determination of acidic pharmaceuticals, antibiotics and ivermectin in river sediment using liquid chromatography-tandem mass spectrometry, J. Chromatogr. A, 1021 (2003) 133-144.

241. Lundström E., Adolfsson-Erici M., Alsberg T., Björlenius B., Eklund B., Lavén M., Breitholtz M. Characterization of additional sewage treatment technologies: Ecotoxicological effects and levels of selected pharmaceuticals, hormones and endocrine disruptors, Ecotoxicol. Environ. Saf., 73 (2010) 1612-1619.

242. Luo Y., Guo W., Ngo H.H., Nghiem L.D., Hai F.I., Zhang J., Liang S., Wang X.C. A review on the occurrence of micropollutants in the aquatic environment and their fate and removal during wastewater treatment, Sci. Total Environ., 473-474 (2014) 619-641.

243. Lv T., Zhang Y., Casas M.E., Carvalho P.N., Arias C.A., Bester K., Brix H. Phytoremediation of imazalil and tebuconazole by four emergent wetland plant species in hydroponic medium, Chemosphere, 148 (2016) 459-466.

244. Ma J., Graham N.J.D. Degradation of atrazine by manganese-catalysed ozonation-influence of radical scavengers, Water Res., 34 (2000) 3822-3828.

245. Ma K., Qin Z., Zhao Z., Zhao C., Liang S. Toxicity evaluation of wastewater collected at different treatment stages from a pharmaceutical industrial park wastewater treatment plant, Chemosphere, 158 (2016) 163-170.

246. Machado F., Boule P. Photonitration and photonitrosation of phenolic derivatives induced in aqueous solution by excitation of nitrite and nitrate ions, J. Photochem. Photobiol. A: Chem., 86 (1995) 73-80.

247. Macova M., Escher B.I., Reungoat J., Carswell S., Chue K.L., Keller J., Mueller J.F. Monitoring the biological activity of micropollutants during advanced wastewater treatment with ozonation and activated carbon filtration, Water Res., 44 (2010) 477-492. 
248. Maeng S.K., Ameda E., Sharma S.K., Grützmacher G., Amy G.L. Organic micropollutant removal from wastewater effluent-impacted drinking water sources during bank filtration and artificial recharge, Water Res., 44 (2010) 4003-4014.

249. Maeng S.K., Sharma S.K., Abel C.D.T., Magic-Knezev A., Amy G.L. Role of biodegradation in the removal of pharmaceutically active compounds with different bulk organic matter characteristics through managed aquifer recharge: Batch and column studies, Water Res., 45 (2011) 4722-4736.

250. Marco-Urrea E., Pérez-Trujillo M., Vicent T., Caminal G. Ability of white-rot fungi to remove selected pharmaceuticals and identification of degradation products of ibuprofen by Trametes versicolor, Chemosphere, 74 (2009) 765-772.

251. Martínez-Hernández V., Meffe R., Herrera S., Arranz E., de Bustamante I. Sorption/desorption of non-hydrophobic and ionisable pharmaceutical and personal care products from reclaimed water onto/from a natural sediment, Sci. Total Environ., 472 (2014) 273-281.

252. Martinez C., Vilarino S., Fernandez M.I., Faria J., Canle M., Santaballa J.A. Mechanism of degradation of ketoprofen by heterogeneous photocatalysis in aqueous solution, Appl. Catal,. B, 142 (2013) 633-646.

253. Martinez C.M., Alvarez L.H., Celis L.B., Cervantes F.J. Humus-reducing microorganisms and their valuable contribution in environmental processes, Appl. Microbiol. Biotechnol., 97 (2013) 10293-10308.

254. Martínez J.L. Antibiotics and antibiotic resistance genes in natural environments, Science, 321 (2008) 365-367.

255. Mashtare M.L., Lee L.S., Nies L.F., Turco R.F. Transformation of 17 $\alpha$-Estradiol, 17 $\beta$-Estradiol, and estrone in sediments under nitrate- and sulfate-reducing conditions, Environ. Sci. Technol., 47 (2013) 7178-7185.

256. Maszkowska J., Stolte S., Kumirska J., Łukaszewicz P., Mioduszewska K., Puckowski A., Caban M., Wagil M., Stepnowski P., Białk-Bielińska A. Beta-blockers in the environment: Part I. Mobility and hydrolysis study, Sci. Total Environ., 493 (2014) 1112-1121.

257. Matamoros V., Garcia J., Bayona J.M. Behavior of selected pharmaceuticals in subsurface flow constructed wetlands: A pilot-scale study, Environ. Sci. Technol., 39 (2005) 5449-5454.

258. Matamoros V., Bayona J.M. Elimination of pharmaceuticals and personal care products in subsurface flow constructed wetlands, Environ. Sci. Technol., 40 (2006) 5811-5816.

259. Matamoros V., Arias C., Brix H., Bayona J.M. Removal of pharmaceuticals and personal care products (PPCPs) from urban wastewater in a pilot vertical flow constructed wetland and a sand filter, Environ. Sci. Technol., 41 (2007) 8171-8177.

260. Matamoros V., Caselles-Osorio A., García J., Bayona J.M. Behaviour of pharmaceutical products and biodegradation intermediates in horizontal subsurface flow constructed wetland. A microcosm experiment, Sci. Total Environ., 394 (2008) 171-176.

261. Matamoros V., García J., Bayona J.M. Organic micropollutant removal in a full-scale surface flow constructed wetland fed with secondary effluent, Water Res., 42 (2008) 653-660. 


\section{Bibliography}

262. Matamoros V., Arias C., Brix H., Bayona J.M. Preliminary screening of small-scale domestic wastewater treatment systems for removal of pharmaceutical and personal care products, Water Res., 43 (2009) 55-62.

263. Matamoros V., Duhec A., Albaiges J., Bayona J.M. Photodegradation of carbamazepine, ibuprofen, ketoprofen and 17 $\alpha$-ethinylestradiol in fresh and seawater, Water Air Soil Pollut., 196 (2009) 161-168.

264. Matamoros V., Hijosa M., Bayona J.M. Assessment of the pharmaceutical active compounds removal in wastewater treatment systems at enantiomeric level. Ibuprofen and naproxen, Chemosphere, 75 (2009) 200-205.

265. Matamoros V., Nguyen L.X., Arias C.A., Salvadó V., Brix H. Evaluation of aquatic plants for removing polar microcontaminants: A microcosm experiment, Chemosphere, 88 (2012) 12571264.

266. Matamoros V., Salvadó V. Evaluation of the seasonal performance of a water reclamation pond-constructed wetland system for removing emerging contaminants, Chemosphere, 86 (2012) 111-117.

267. Matamoros V., Gutiérrez R., Ferrer I., García J., Bayona J.M. Capability of microalgae-based wastewater treatment systems to remove emerging organic contaminants: A pilot-scale study, J Hazard. Mater., 288 (2015) 34-42.

268. Mayer P., Cuhel R., Nyholm N. A simple in vitro fluorescence method for biomass measurements in algal growth inhibition tests, Water Res., 31 (1997) 2525-2531.

269. McCutcheon S., Schnoor J. Overview of phytotransformation and control of wastes, Phytoremediation: Transformation and control of contaminants, 358 (2003).

270. Mei Fun Choong A., Lay-Ming Teo S., Lene Leow J., Ling Koh H., Chi Lui Ho P. A preliminary ecotoxicity study of pharmaceuticals in the marine environment, J. Toxicol. Environ. Health, A, 69 (2006) 1959-1970.

271. Meng F., Chae S.-R., Drews A., Kraume M., Shin H.-S., Yang F. Recent advances in membrane bioreactors (MBRs): Membrane fouling and membrane material, Water Res., 43 (2009) 1489-1512.

272. Meng P., Pei H., Hu W., Shao Y., Li Z. How to increase microbial degradation in constructed wetlands: Influencing factors and improvement measures, Bioresour. Technol., 157 (2014) 316326.

273. Meßner B., Thulke O., Schäffner A.R. Arabidopsis glucosyltransferases with activities toward both endogenous and xenobiotic substrates, Planta, 217 (2003) 138-146.

274. Miller E.L., Nason S.L., Karthikeyan K.G., Pedersen J.A. Root uptake of pharmaceuticals and personal care product ingredients, Environ. Sci. Technol., 50 (2015) 525-541.

275. Miralles-Cuevas S., Oller I., Ruiz Aguirre A., Sánchez Pérez J.A., Malato Rodríguez S. Removal of pharmaceuticals at microg $\mathrm{L}-1$ by combined nanofiltration and mild solar photoFenton, Chem. Eng. J., 239 (2014) 68-74.

276. Miranda-García N., Suárez S., Sánchez B., Coronado J.M., Malato S., Maldonado M.I. Photocatalytic degradation of emerging contaminants in municipal wastewater treatment plant effluents using immobilized TiO2 in a solar pilot plant, Appl. Catal,. B, 103 (2011) 294-301. 
277. Monteiro S.C., Boxall A.B.A. Factors affecting the degradation of pharmaceuticals in agricultural soils, Environ. Toxicol. Chem., 28 (2009) 2546-2554.

278. Murk A., Vethaak A., Rijs G., Schrap S., Ruiter H., Gerritsen A., Lahr J. Estrogens and xenoestrogens in the aquatic environment of The Netherlands: occurence, potency and biological effects, in, Rijkswaterstaat, RIKZ, 2002.

279. Muziasari W.I., Managaki S., Pärnänen K., Karkman A., Lyra C., Tamminen M., Suzuki S., Virta M. Sulphonamide and trimethoprim resistance genes persist in sediments at Baltic Sea aquaculture farms but are not detected in the surrounding environment, PLoS One, 9 (2014) e92702.

280. Nguyen L.M. Organic matter composition, microbial biomass and microbial activity in gravelbed constructed wetlands treating farm dairy wastewaters, Ecol. Eng., 16 (2000) 199-221.

281. Nitz G.M., Schnitzler W.H. Effect of PAR and UV-B radiation on the quality and quantity of the essential oil in sweet basil (Ocimum basilicum L.), in: Acta Horticulturae, International Society for Horticultural Science (ISHS), Leuven, Belgium, 2004, pp. 375-381.

282. Nõlvak H., Truu M., Tiirik K., Oopkaup K., Sildvee T., Kaasik A., Mander Ü., Truu J. Dynamics of antibiotic resistance genes and their relationships with system treatment efficiency in a horizontal subsurface flow constructed wetland, Sci. Total Environ., 461 (2013) 636-644.

283. Oaks J.L., Gilbert M., Virani M.Z., Watson R.T., Meteyer C.U., Rideout B.A., Shivaprasad H.L., Ahmed S., Iqbal Chaudhry M.J., Arshad M., Mahmood S., Ali A., Ahmed Khan A. Diclofenac residues as the cause of vulture population decline in Pakistan, Nature, 427 (2004) 630-633.

284. Oller I., Malato S., Sanchez-Perez J.A. Combination of advanced oxidation processes and biological treatments for wastewater decontamination-A review, Sci. Total Environ., 409 (2011) 4141-4166.

285. Olry A., Schneider-Belhaddad F., Heintz D., Werck-Reichhart D. A medium-throughput screening assay to determine catalytic activities of oxygen-consuming enzymes: a new tool for functional characterization of cytochrome P450 and other oxygenases, The Plant Journal, 51 (2007) 331-340.

286. Papoulis D., Komarneni S., Panagiotaras D., Stathatos E., Christoforidis K.C., FernándezGarcía M., Li H., Shu Y., Sato T., Katsuki H. Three-phase nanocomposites of two nanoclays and $\mathrm{TiO}_{2}$ : Synthesis, characterization and photacatalytic activities, Appl. Catal,. B, 147 (2014) 526-533.

287. Park N., Vanderford B.J., Snyder S.A., Sarp S., Kim S.D., Cho J. Effective controls of micropollutants included in wastewater effluent using constructed wetlands under anoxic condition, Ecol. Eng., 35 (2009) 418-423.

288. Pawłowska B., Biczak R. Evaluation of the effect of tetraethylammonium bromide and chloride on the growth and development of terrestrial plants, Chemosphere, 149 (2016) 24-33.

289. Pereira V.J., Linden K.G., Weinberg H.S. Evaluation of UV irradiation for photolytic and oxidative degradation of pharmaceutical compounds in water, Water Res., 41 (2007) 4413-4423.

290. Pereira V.J., Weinberg H.S., Linden K.G., Singer P.C. UV degradation kinetics and modeling of pharmaceutical compounds in laboratory grade and surface water via direct and indirect photolysis at $254 \mathrm{~nm}$, Environ. Sci. Technol., 41 (2007) 1682-1688. 


\section{Bibliography}

291. Pflugmacher S., Sandermann H. Cytochrome P450 monooxygenases for fatty acids and xenobiotics in marine macroalgae, Plant Physiol., 117 (1998) 123-128.

292. Piecha M., Sarakha M., Trebse P. Photocatalytic degradation of cholesterol-lowering statin drugs by $\mathrm{TiO}_{2}$-based catalyst. Kinetics, analytical studies and toxicity evaluation, J. Photochem. Photobiol. A: Chem., 213 (2010) 61-69.

293. Pietrini F., Di Baccio D., Aceña J., Pérez S., Barceló D., Zacchini M. Ibuprofen exposure in Lemna gibba L.: Evaluation of growth and phytotoxic indicators, detection of ibuprofen and identification of its metabolites in plant and in the medium, J. Hazard. Mater., 300 (2015) 189193.

294. Popple T., Williams J.B., May E., Mills G.A., Oliver R. Evaluation of a sequencing batch reactor sewage treatment rig for investigating the fate of radioactively labelled pharmaceuticals: Case study of propranolol, Water Res., 88 (2016) 83-92.

295. Proia L., von Schiller D., Sànchez-Melsió A., Sabater S., Borrego C.M., Rodríguez-Mozaz S., Balcázar J.L. Occurrence and persistence of antibiotic resistance genes in river biofilms after wastewater inputs in small rivers, Environ. Pollut., 210 (2016) 121-128.

296. Pruden A., Pei R., Storteboom H., Carlson K.H. Antibiotic resistance genes as emerging contaminants: Studies in northern Colorado, Environmental Science and Technology, 40 (2006) 7445-7450.

297. Purdom C.E., Hardiman P.A., Bye V.V.J., Eno N.C., Tyler C.R., Sumpter J.P. Estrogenic effects of effluents from sewage treatment works, Chem. Ecol., 8 (1994) 275-285.

298. Quintana J.B., Weiss S., Reemtsma T. Pathways and metabolites of microbial degradation of selected acidic pharmaceutical and their occurrence in municipal wastewater treated by a membrane bioreactor, Water Res., 39 (2005) 2654-2664.

299. Radke M., Maier M.P. Lessons learned from water/sediment-testing ofpharmaceuticals, Water Res., 55 (2014) 63-73.

300. Ramil M., El Aref T., Fink G., Scheurer M., Ternes T.A. Fate of beta blockers in aquaticsediment systems: sorption and biotransformation, Environ. Sci. Technol., 44 (2010) 962-970.

301. Rattier M., Reungoat J., Keller J., Gernjak W. Removal of micropollutants during tertiary wastewater treatment by biofiltration: Role of nitrifiers and removal mechanisms, Water Res., 54 (2014) 89-99.

302. Rauch-Williams T., Hoppe-Jones C., Drewes J.E. The role of organic matter in the removal of emerging trace organic chemicals during managed aquifer recharge, Water Res., 44 (2010) 449460 .

303. Real F.J., Benitez J.F., Acero J.L., Casas F. Comparison between chlorination and ozonation treatments for the elimination of the emerging contaminants amitriptyline hydrochloride, methyl salicylate and 2-phenoxyethanol in surface waters and secondary effluents, J. Chem. Technol. Biotechnol., 90 (2015) 1400-1407.

304. Reinhard M., Shang S., Kitanidis P.K., Orwin E., Hopkins G.D., LeBron C.A. In situ BTEX biotransformation under enhanced nitrate- and sulfate-reducing conditions, Environ. Sci. Technol., 31 (1997) 28-36. 
305. Richards S., Withers P.J.A., Paterson E., McRoberts C.W., Stutter M. Removal and attenuation of sewage effluent combined tracer signals of phosphorus, caffeine and saccharin in soil, Environ. Pollut., 223 (2017) 277-285.

306. Rivera-Utrilla J., Sánchez-Polo M., Ferro-García M.Á., Prados-Joya G., Ocampo-Pérez R. Pharmaceuticals as emerging contaminants and their removal from water. A review, Chemosphere, 93 (2013) 1268-1287.

307. Rizzo L., Meric S., Guida M., Kassinos D., Belgiorno V. Heterogenous photocatalytic degradation kinetics and detoxification of an urban wastewater treatment plant effluent contaminated with pharmaceuticals, Water Res., 43 (2009) 4070-4078.

308. Rizzo L., Meric S., Kassinos D., Guida M., Russo F., Belgiorno V. Degradation of diclofenac by $\mathrm{TiO}_{2}$ photocatalysis: $\mathrm{UV}$ absorbance kinetics and process evaluation through a set of toxicity bioassays, Water Res., 43 (2009) 979-988.

309. Rodriguez-Mozaz S., Chamorro S., Marti E., Huerta B., Gros M., Sànchez-Melsió A., Borrego C.M., Barceló D., Balcázar J.L. Occurrence of antibiotics and antibiotic resistance genes in hospital and urban wastewaters and their impact on the receiving river, Water Res., 69 (2015) 234-242.

310. Roh H., Subramanya N., Zhao F., Yu C.-P., Sandt J., Chu K.-H. Biodegradation potential of wastewater micropollutants by ammonia-oxidizing bacteria, Chemosphere, 77 (2009) 10841089.

311. Rühmland S., Wick A., Ternes T.A., Barjenbruch M. Fate of pharmaceuticals in a subsurface flow constructed wetland and two ponds, Ecol. Eng., 80 (2015) 125-139.

312. Salt D.E., Smith R.D., Raskin I. Phytoremediation, Annu. Rev. Plant Physiol. Plant Mol. Biol., 49 (1998) 643-668.

313. Sandermann H., Diesperger H., Scheel D. Metabolism of xenobiotics by plant cell cultures, in: W. Barz, E. Reinhard, M.H. Zenk (Eds.) Plant tissue culture and its bio-technological application. Proceedings in Life Sciences., Springer, Berlin, Heidelberg, 1977, pp. 178-196.

314. Sandermann H. Plant metabolism of organic xenobiotics. status and prospects of the 'green liver' concept, in: A. Altman, M. Ziv, S. Izhar (Eds.) Plant Biotechnology and In Vitro Biology in the 21st Century: Proceedings of the IXth International Congress of the International Association of Plant Tissue Culture and Biotechnology Jerusalem, Israel, 14-19 June 1998, Springer Netherlands, Dordrecht, 1999, pp. 321-328.

315. Santos J.L., Aparicio I., Alonso E., Callejón M. Simultaneous determination of pharmaceutically active compounds in wastewater samples by solid phase extraction and highperformance liquid chromatography with diode array and fluorescence detectors, Anal. Chim. Acta, 550 (2005) 116-122.

316. Santos J.L., Aparicio I., Alonso E. Occurrence and risk assessment of pharmaceutically active compounds in wastewater treatment plants. A case study: Seville city (Spain), Environ. Int., 33 (2007) 596-601.

317. Schaffer M., Börnick H., Nödler K., Licha T., Worch E. Role of cation exchange processes on the sorption influenced transport of cationic $\beta$-blockers in aquifer sediments, Water Res., 46 (2012) 5472-5482. 


\section{Bibliography}

318. Schaffer M., Kröger K.F., Nödler K., Ayora C., Carrera J., Hernández M., Licha T. Influence of a compost layer on the attenuation of 28 selected organic micropollutants under realistic soil aquifer treatment conditions: Insights from a large scale column experiment, Water Res., 74 (2015) 110-121.

319. Schriewer A., Wehlmann A., Wuertz S. Improving qPCR efficiency in environmental samples by selective removal of humic acids with DAX-8, J. Microbiol. Methods, 85 (2011) 16-21.

320. Schröder P., Juuti S., Roy S., Sandermann H., Sutinen S. Exposure to chlorinated acetic acids: responses of peroxidase and glutathione S-transferase activity in pine needles, Environ. Sci. Pollut. Res., 4 (1997) 163-171.

321. Schröder P., Maier H., Debus R. Detoxification of herbicides in phragmites australis, Zeitschrift Fur Naturforschung Section C-A Journal of Biosciences, 60 (2005) 317-324.

322. Schröder P. Exploiting plant metabolism for the phytoremediation of organic xenobiotics, in: $\mathrm{N}$. Willey (Ed.) Phytoremediation: Methods and Reviews, Humana Press, Totowa, NJ, 2007, pp. 251-263.

323. Schulze T., Weiss S., Schymanski E., von der Ohe P.C., Schmitt-Jansen M., Altenburger R., Streck G., Brack W. Identification of a phytotoxic photo-transformation product of diclofenac using effect-directed analysis, Environ. Pollut., 158 (2010) 1461-1466.

324. Sebastine I.M., Wakeman R.J. Consumption and environmental hazards of pharmaceutical substances in the UK, Process Saf. Environ. Prot., 81 (2003) 229-235.

325. Semrany S., Favier L., Djelal H., Taha S., Amrane A. Bioaugmentation: Possible solution in the treatment of Bio-Refractory Organic Compounds (Bio-ROCs), Biochem. Eng. J., 69 (2012) 7586.

326. Sheahan D., Bucke D., Matthiessen P., Sumpter J., Kirby M., Neall P., Waldock M. The effects of low levels of 17a-ethynylestradiol upon plasma vitellogenin levels in male and female rainbow trout, Oncorhynchus mykiss held at two acclimation temperatures, (1994).

327. Shehabi A., Odeh J., Fayyad M. Characterization of antimicrobial resistance and class 1 integrons found in Escherichia coli isolates from human stools and drinking water sources in Jordan, J. Chemother., 18 (2006) 468-472.

328. Shemer H., Kunukcu Y.K., Linden K.G. Degradation of the pharmaceutical Metronidazole via UV, Fenton and photo-Fenton processes, Chemosphere, 63 (2006) 269-276.

329. Silva B.F.d., Jelic A., López-Serna R., Mozeto A.A., Petrovic M., Barceló D. Occurrence and distribution of pharmaceuticals in surface water, suspended solids and sediments of the Ebro river basin, Spain, Chemosphere, 85 (2011) 1331-1339.

330. Sköld O. Sulfonamide resistance: mechanisms and trends, Drug Resistance Updates, 3 (2000) 155-160.

331. Snyder S.A., Adham S., Redding A.M., Cannon F.S., DeCarolis J., Oppenheimer J., Wert E.C., Yoon Y. Role of membranes and activated carbon in the removal of endocrine disruptors and pharmaceuticals, Desalination, 202 (2007) 156-181.

332. Spain J.C., Pritchard P., Bourquin A. Effects of adaptation on biodegradation rates in sediment/water cores from estuarine and freshwater environments, Appl. Environ. Microbiol., 40 (1980) 726-734. 
333. Stamatelatou K., Frouda C., Fountoulakis M.S., Drillia P., Kornaros M., Lyberatos G. Pharmaceuticals and health care products in wastewater effluents: the example of carbamazepine, Water Science and Technology: Water Supply, 3 (2003) 131-137.

334. Stottmeister U., Wießner A., Kuschk P., Kappelmeyer U., Kästner M., Bederski O., Müller R.A., Moormann H. Effects of plants and microorganisms in constructed wetlands for wastewater treatment, Biotechnol. Adv., 22 (2003) 93-117.

335. Straathof A.L. Explorations of soil microbial processes driven by dissolved organic carbon, Wageningen University, 2015.

336. Straathof A.L., Comans R.N.J. Input materials and processing conditions control compost dissolved organic carbon quality, Bioresour. Technol., 179 (2015) 619-623.

337. Suarez M.P., Rifai H.S. Biodegradation rates for fuel hydrocarbons and chlorinated solvents in groundwater, Biorem. J., 3 (1999) 337-362.

338. Sun Q., Deng S., Huang J., Shen G., Yu G. Contributors to estrogenic activity in wastewater from a large wastewater treatment plant in Beijing, China, Environ. Toxicol. Pharmacol., 25 (2008) 20-26.

339. Susarla S., Medina V.F., McCutcheon S.C. Phytoremediation: An ecological solution to organic chemical contamination, Ecol. Eng., 18 (2002) 647-658.

340. Suzuki S., Ogo M., Miller T., Shimizu A., Takada H., Siringan M.A. Who possesses drug resistance genes in the aquatic environment?: sulfamethoxazole (SMX) resistance genes among the bacterial community in water environment of Metro-Manila, Philippines, Frontiers in Microbiology, 4 (2013).

341. Szabo R.K., Megyeri C., Illes E., Gajda-Schrantz K., Mazellier P., Dombi A. Phototransformation of ibuprofen and ketoprofen in aqueous solutions, Chemosphere, 84 (2011) 1658-1663.

342. Tekin H., Bilkay O., Ataberk S.S., Balta T.H., Ceribasi I.H., Sanin F.D., Dilek F.B., Yetis U. Use of Fenton oxidation to improve the biodegradability of a pharmaceutical wastewater, J. Hazard. Mater., 136 (2006) 258-265.

343. Tello A., Austin B., Telfer T.C. Selective pressure of antibiotic pollution on bacteria of importance to public health, Environ. Health Perspect., 120 (2012) 1100-1106.

344. Theodoulou F.L. Plant ABC transporters, Biochim. Biophys. Acta, 1465 (2000) 79-103.

345. Tixier C., Singer H.P., Oellers S., Müller S.R. Occurrence and fate of carbamazepine, clofibric acid, diclofenac, ibuprofen, ketoprofen, and naproxen in surface waters, Environ. Sci. Technol., 37 (2003) 1061-1068.

346. Tran N.H., Urase T., Kusakabe O. The characteristics of enriched nitrifier culture in the degradation of selected pharmaceutically active compounds, J. Hazard. Mater., 171 (2009) 1051-1057.

347. Traversa A., Loffredo E., Gattullo C., Palazzo A., Bashore T., Senesi N. Comparative evaluation of compost humic acids and their effects on the germination of switchgrass (Panicum vigatum L.), J. Soils Sed., 14 (2014) 432-440. 


\section{Bibliography}

348. Umar M., Aziz H.A., Yusoff M.S. Trends in the use of Fenton, electro-Fenton and photoFenton for the treatment of landfill leachate, Waste Manage. (Oxford), 30 (2010) 2113-2121.

349. Välitalo P., Perkola N., Seiler T.-B., Sillanpää M., Kuckelkorn J., Mikola A., Hollert H., Schultz E. Estrogenic activity in Finnish municipal wastewater effluents, Water Res., 88 (2016) 740-749.

350. Van Boeckel T.P., Gandra S., Ashok A., Caudron Q., Grenfell B.T., Levin S.A., Laxminarayan R. Global antibiotic consumption 2000 to 2010: an analysis of national pharmaceutical sales data, The Lancet Infectious Diseases, 14 (2014) 742-750.

351. Van den Boomen R., Kampf R., Foekema E. Waterharmonica's in nederland-1996-2011: van effluent tot bruikbaar oppervlaktewater, Stowa, 2012.

352. Van der Aa N.G.F.M., Kommer G.J., van Montfoort J.E., Versteegh J.F.M. Demographic projections of future pharmaceutical consumption in the Netherlands, Water Sci. Technol., 63 (2011) 825-831.

353. Van Trump J.I., Sun Y., Coates J.D. Microbial interactions with humic substances, in: Adv. Appl. Microbiol., Academic Press, 2006, pp. 55-96.

354. Van Zomeren A., Comans R.N.J. Measurement of humic and fulvic acid concentrations and dissolution properties by a rapid batch procedure, Environ. Sci. Technol., 41 (2007) 6755-6761.

355. Vanacker H., Carver T.L.W., Foyer C.H. Pathogen-induced changes in the antioxidant status of the apoplast in barley leaves, Plant Physiol., 117 (1998) 1103-1114.

356. Vanek T., Podlipna R., Fialova Z., Petrova S., Soudek P. Uptake of xenobiotics from polluted waters by plants, in: D. Fatta-Kassinos, K. Bester, K. Kümmerer (Eds.) Xenobiotics in the urban water cycle: Mass flows, environmental processes, mitigation and treatment strategies, Springer Netherlands, Dordrecht, 2010, pp. 431-444.

357. Venkata Subba Rao K., Rachel A., Subrahmanyam M., Boule P. Immobilization of $\mathrm{TiO}_{2}$ on pumice stone for the photocatalytic degradation of dyes and dye industry pollutants, Appl. Catal,. B, 46 (2003) 77-85.

358. Verkleij J.A.C., Golan-Goldhirsh A., Antosiewisz D.M., Schwitzguébel J.-P., Schröder P. Dualities in plant tolerance to pollutants and their uptake and translocation to the upper plant parts, Environ. Exp. Bot., 67 (2009) 10-22.

359. Verlicchi P., Al Aukidy M., Zambello E. Occurrence of pharmaceutical compounds in urban wastewater: Removal, mass load and environmental risk after a secondary treatment-A review, Sci. Total Environ., 429 (2012) 123-155.

360. Verlicchi P., Galletti A., Petrovic M., Barceló D., Al Aukidy M., Zambello E. Removal of selected pharmaceuticals from domestic wastewater in an activated sludge system followed by a horizontal subsurface flow bed - Analysis of their respective contributions, Sci. Total Environ., 454-455 (2013) 411-425.

361. Verlicchi P., Zambello E. How efficient are constructed wetlands in removing pharmaceuticals from untreated and treated urban wastewaters? A review, Sci. Total Environ., 470-471 (2014) 1281-1306. 
362. Verlicchi P., Zambello E. How efficient are constructed wetlands in removing pharmaceuticals from untreated and treated urban wastewaters? A review, Sci. Total Environ., 470-471 (2014) 1281-1306.

363. Verliefde A., Cornelissen E., Amy G., Van der Bruggen B., van Dijk H. Priority organic micropollutants in water sources in Flanders and the Netherlands and assessment of removal possibilities with nanofiltration, Environ. Pollut., 146 (2007) 281-289.

364. Vialaton D., Richard C. Phototransformation of aromatic pollutants in solar light: Photolysis versus photosensitized reactions under natural water conditions, Aquat. Sci., 64 (2002) 207-215.

365. Vieno N., Tuhkanen T., Kronberg L. Elimination of pharmaceuticals in sewage treatment plants in Finland, Water Res., 41 (2007) 1001-1012.

366. Vilhunen S., Bosund M., Kääriäinen M.-L., Cameron D., Sillanpää M. Atomic layer deposited $\mathrm{TiO}_{2}$ films in photodegradation of aqueous salicylic acid, Sep. Purif. Technol., 66 (2009) 130134.

367. Villeneuve D.L., Blankenship A.L., Giesy J.P. Derivation and application of relative potency estimates based on in vitro bioassay results, Environ. Toxicol. Chem., 19 (2000) 2835-2843.

368. Vulava V.M., Cory W.C., Murphey V.L., Ulmer C.Z. Sorption, photodegradation, and chemical transformation of naproxen and ibuprofen in soils and water, Sci. Total Environ., 565 (2016) 1063-1070.

369. Vymazal J. The use of sub-surface constructed wetlands for wastewater treatment in the Czech Republic: 10 years experience, Ecol. Eng., 18 (2002) 633-646.

370. Vymazal J. Removal of nutrients in various types of constructed wetlands, Sci. Total Environ., 380 (2007) 48-65.

371. Wang S., Seiwert B., Kästner M., Miltner A., Schäffer A., Reemtsma T., Yang Q., Nowak K.M. (Bio)degradation of glyphosate in water-sediment microcosms - A stable isotope co-labeling approach, Water Res., 99 (2016) 91-100.

372. Wei L.-L., Zhao Q.-L., Xue S., Jia T., Tang F., You P.-Y. Behavior and characteristics of DOM during a laboratory-scale horizontal subsurface flow wetland treatment: Effect of DOM derived from leaves and roots, Ecol. Eng., 35 (2009) 1405-1414.

373. Wenk J., von Gunten U., Canonica S. Effect of dissolved organic matter on the transformation of contaminants induced by excited triplet states and the hydroxyl radical, Environ. Sci. Technol., 45 (2011) 1334-1340.

374. Wenk J., Eustis S.N., McNeill K., Canonica S. Quenching of excited triplet states by dissolved natural organic matter, Environ. Sci. Technol., 47 (2013) 12802-12810.

375. Wilt A.D., He Y., Sutton N.B., Langenhoff A.A.M., Rijnaarts H.H.M. Influence of redox conditions on sorption and biodegradation of pharmaceuticals in sediment batch and column experiments, In submission, (2017).

376. Wu D., Huang Z., Yang K., Graham D., Xie B. Relationships between antibiotics and antibiotic resistance gene levels in municipal solid waste leachates in Shanghai, China, Environ. Sci. Technol., 49 (2015) 4122-4128. 


\section{Bibliography}

377. Wu H., Fan J., Zhang J., Ngo H.H., Guo W., Liang S., Hu Z., Liu H. Strategies and techniques to enhance constructed wetland performance for sustainable wastewater treatment, Environ. Sci. Pollut. Res., 22 (2015) 14637-14650.

378. Wu H., Zhang J., Ngo H.H., Guo W., Hu Z., Liang S., Fan J., Liu H. A review on the sustainability of constructed wetlands for wastewater treatment: Design and operation, Bioresour. Technol., 175 (2015) 594-601.

379. Wu X., Dodgen L.K., Conkle J.L., Gan J. Plant uptake of pharmaceutical and personal care products from recycled water and biosolids: a review, Sci. Total Environ., 536 (2015) 655-666.

380. Xia Q.B., Li Z., Xi H.X., Xu K.F. Activation energy for dibenzofuran desorption from $\mathrm{Fe}^{3+} / \mathrm{TiO}_{2}$ and $\mathrm{Ce}^{3+} / \mathrm{TiO}_{2}$ photocatalysts coated onto glass fibres, Adsorpt. Sci. Technol., 23 (2005) 357-366.

381. Xu J., Wu L., Chang A.C. Degradation and adsorption of selected pharmaceuticals and personal care products (PPCPs) in agricultural soils, Chemosphere, 77 (2009) 1299-1305.

382. Xu Y., Nguyen T.V., Reinhard M., Gin K.Y.-H. Photodegradation kinetics of p-tert-octylphenol, 4-tert-octylphenoxy-acetic acid and ibuprofen under simulated solar conditions in surface water, Chemosphere, 85 (2011) 790-796.

383. Xu Y., Guo C., Luo Y., Lv J., Zhang Y., Lin H., Wang L., Xu J. Occurrence and distribution of antibiotics, antibiotic resistance genes in the urban rivers in Beijing, China, Environ. Pollut., 213 (2016) 833-840.

384. Xu Y., Yuan Z., Ni B.-J. Biotransformation of pharmaceuticals by ammonia oxidizing bacteria in wastewater treatment processes, Sci. Total Environ., 566-567 (2016) 796-805.

385. Xue W., Wu C., Xiao K., Huang X., Zhou H., Tsuno H., Tanaka H. Elimination and fate of selected micro-organic pollutants in a full-scale anaerobic/anoxic/aerobic process combined with membrane bioreactor for municipal wastewater reclamation, Water Res., 44 (2010) 59996010 .

386. Yamamoto H., Nakamura Y., Moriguchi S., Nakamura Y., Honda Y., Tamura I., Hirata Y., Hayashi A., Sekizawa J. Persistence and partitioning of eight selected pharmaceuticals in the aquatic environment: Laboratory photolysis, biodegradation, and sorption experiments, Water Res., 43 (2009) 351-362.

387. Yan C., Nie M., Yang Y., Zhou J., Liu M., Baalousha M., Lead J.R. Effect of colloids on the occurrence, distribution and photolysis of emerging organic contaminants in wastewaters, J. Hazard. Mater., 299 (2015) 241-248.

388. Yangali-Quintanilla V., Maeng S.K., Fujioka T., Kennedy M., Li Z., Amy G. Nanofiltration vs. reverse osmosis for the removal of emerging organic contaminants in water reuse, Desalination and Water Treatment, 34 (2011) 50-56.

389. Ye F., Li Y. Enhancement of nitrogen removal in towery hybrid constructed wetland to treat domestic wastewater for small rural communities, Ecol. Eng., 35 (2009) 1043-1050.

390. Yi T., Harper W.F. The link between nitrification and biotransformation of $17 \alpha$-ethinylestradiol, Environ. Sci. Technol., 41 (2007) 4311-4316. 
391. Yu X., Zuo J., Tang X., Li R., Li Z., Zhang F. Toxicity evaluation of pharmaceutical wastewaters using the alga Scenedesmus obliquus and the bacterium Vibrio fischeri, J. Hazard. Mater., 266 (2014) 68-74.

392. Yu Y., Liu Y., Wu L. Sorption and degradation of pharmaceuticals and personal care products (PPCPs) in soils, Environ. Sci. Pollut. Res., 20 (2013) 4261-4267.

393. Zepp R.G., Cline D.M. Rates of direct photolysis in aquatic environment, Environ. Sci. Technol., 11 (1977) 359-366.

394. Zhang D.Q., Gersberg R.M., Hua T., Zhu J.F., Tuan N.A., Tan S.K. Pharmaceutical removal in tropical subsurface flow constructed wetlands at varying hydraulic loading rates, Chemosphere, 87 (2012) 273-277.

395. Zhang D.Q., Gersberg R.M., Zhu J.F., Hua T., Jinadasa K.B.S.N., Tan S.K. Batch versus continuous feeding strategies for pharmaceutical removal by subsurface flow constructed wetland, Environ. Pollut., 167 (2012) 124-131.

396. Zhang D.Q., Hua T., Gersberg R.M., Zhu J., Ng W.J., Tan S.K. Fate of diclofenac in wetland mesocosms planted with Scirpus validus, Ecol. Eng., 49 (2012) 59-64.

397. Zhang D.Q., Gersberg R.M., Hua T., Zhu J.F., Goyal M.K., Ng W.J., Tan S.K. Fate of pharmaceutical compounds in hydroponic mesocosms planted with Scirpus validus, Environ. Pollut., 181 (2013) 98-106.

398. Zhang D.Q., Hua T., Gersberg R.M., Zhu J.F., Ng W.J., Tan S.K. Carbamazepine and naproxen: Fate in wetland mesocosms planted with Scirpus validus, Chemosphere, 91 (2013) 14-21.

399. Zhang D.Q., Hua T., Gersberg R.M., Zhu J.F., Ng W.J., Tan S.K. Fate of caffeine in mesocosms wetland planted with Scirpus validus, Chemosphere, 90 (2013) 1568-1572.

400. Zhang D.Q., Gersberg R.M., Ng W.J., Tan S.K. Removal of pharmaceuticals and personal care products in aquatic plant-based systems: a review, Environ. Pollut., 184 (2014) 620-639.

401. Zhang J., Kirkham M.B. Antioxidant responses to drought in sunflower and sorghum seedlings, New Phytol., 132 (1996) 361-373.

402. Zhang J., Li Z., Ge G., Sun W., Liang Y., Wu L. Impacts of soil organic matter, pH and exogenous copper on sorption behavior of norfloxacin in three soils, Journal of Environmental Sciences, 21 (2009) 632-640.

403. Zhang L., Hendrickx T.L.G., Kampman C., Temmink H., Zeeman G. Co-digestion to support low temperature anaerobic pretreatment of municipal sewage in a UASB-digester, Bioresour. Technol., 148 (2013) 560-566.

404. Zhang S., Lin W., Yu X. Effects of full-scale advanced water treatment on antibiotic resistance genes in the Yangtze Delta area in China, FEMS Microbiol. Ecol., 92 (2016) 1-8.

405. Zhang T., Xu D., He F., Zhang Y., Wu Z. Application of constructed wetland for water pollution control in China during 1990-2010, Ecol. Eng., 47 (2012) 189-197.

406. Zhang T., Wu B., Sun N., Ye Y., Chen H. Sorption and degradation of wastewater-associated pharmaceuticals and personal care products in agricultural soils and sediment, Water Sci. Technol., 68 (2013) 991-998. 


\section{Bibliography}

407. Zhang X.-X., Zhang T., Fang H.H.P. Antibiotic resistance genes in water environment, Appl. Microbiol. Biotechnol., 82 (2009) 397-414.

408. Zhang Y., Geißen S.-U., Gal C. Carbamazepine and diclofenac: Removal in wastewater treatment plants and occurrence in water bodies, Chemosphere, 73 (2008) 1151-1161.

409. Zhang Y., Lv T., Carvalho P.N., Arias C.A., Chen Z., Brix H. Removal of the pharmaceuticals ibuprofen and iohexol by four wetland plant species in hydroponic culture: plant uptake and microbial degradation, Environ. Sci. Pollut. Res., 23 (2015) 2890-2898.

410. Zhang Y., Price G.W., Jamieson R., Burton D., Khosravi K. Sorption and desorption of selected non-steroidal anti-inflammatory drugs in an agricultural loam-textured soil, Chemosphere, 174 (2017) 628-637.

411. Zhao C., Xie H., Mu Y., Xu X., Zhang J., Liu C., Liang S., Ngo H.H., Guo W., Xu J., Wang Q. Bioremediation of endosulfan in laboratory-scale constructed wetlands: effect of bioaugmentation and biostimulation, Environ. Sci. Pollut. Res., 21 (2014) 12827-12835.

412. Zhu Y.-G., Johnson T.A., Su J.-Q., Qiao M., Guo G.-X., Stedtfeld R.D., Hashsham S.A., Tiedje J.M. Diverse and abundant antibiotic resistance genes in Chinese swine farms, Proceedings of the National Academy of Sciences, 110 (2013) 3435-3440.

413. Zwiener C., Frimmel F.H. Short-term tests with a pilot sewage plant and biofilm reactors for the biological degradation of the pharmaceutical compounds clofibric acid, ibuprofen, and diclofenac, Sci. Total Environ., 309 (2003) 201-211. 
Summary 


\section{Summary}

Pharmaceutically active compounds (PhACs) are a class of manmade chemicals to target metabolic and molecular pathways in humans and animals. Due to manufacturing processes, improper disposal and metabolic excretion, the widely used PhACs are continuously released into the aquatic environment and as a result exhibit a pseudo-persistent behaviour. Although concentrations of PhACs in the environment are at trace-level, they could cause a significant cumulative effect on the metabolism of non-target organisms and the ecosystems as a whole. Wastewater discharge is an important transport pathway of PhACs to the aquatic environment. However, conventional wastewater treatment plants (WWTPs) designed for removing sewage organic matter and nutrients do not remove PhACs sufficiently.

To further eliminate PhACs in the wastewater effluent, post-treatment processes urgently need to be established. Constructed wetland $(\mathrm{CW})$ is an environmentally friendly and cost efficient post-treatment technique. In the last decade, CWs have been found to remove PhACs through a combination of removal processes which can be seen as functional tools, mainly photodegradation, sorption, (aerobic/anaerobic) biodegradation, and phytoremediation. In principle, $\mathrm{CWs}$ are an integrated and enhanced version of natural ecosystems for $\mathrm{PhAC}$ removal. To date, relevant studies mainly focus on investigation of PhAC removal in $\mathrm{CWs}$ and optimization of the removal by manipulating operational parameters. In comparison, much less is known about the removal mechanisms themselves, and thus CWs are generally viewed as a "black box" due to the complex interplay of various removal processes. To this end, this thesis investigated removal mechanisms of PhACs in individual processes, and fate of PhACs in a whole CW. Moreover, important environmental factors (i.e. natural organic matter, redox conditions) were studied and recommendations to enhance removal processes were proposed for more effective PhAC removal in CWs.

In Chapter 2, a combination of chemical, toxicological, and molecular analyses were employed to evaluate the attenuation of PhACs, toxic potency and antibiotic resistance genes (ARGs) in existing CWs serving as post-treatment. $17 \mathrm{PhACs}$ from various categories, including anti-inflammatory drugs, antibiotics, $\beta$-blockers, lipid regulators, psychiatric drugs, simulants, estrogenic compounds, were included in the target list. Chemical analysis showed that 14 out of the $17 \mathrm{PhACs}$ were detected in the three local CWs investigated. Seven PhACs were found to be discharged to the surface water at concentrations higher than $0.1 \mu \mathrm{g} / \mathrm{l}$, which is the guideline value adopted by toxicologists for drinking water. The overall median 
removal of the $14 \mathrm{PhACs}$ was approximately $50 \%$ in the vertical subsurface flow $\mathrm{CW}$, while the removal in the other two free water surface flow CWs was negligible. Toxicological analysis demonstrated that the toxic potency of wastewater extracts is of environmental concern. The observed estrogenicity was attenuated in CWs while the neurotoxic potency of wastewater extracts did not decrease after $\mathrm{CW}$ treatment. Molecular analysis indicated high abundance of ARGs in CWs. In general CWs showed positive removal for ARGs but ARG regrowth was observed during $\mathrm{CW}$ treatment. Finally, multivariate analysis showed that most $\mathrm{PhACs}$ are positively correlated to the observed toxic potency. Low removal of organics and nutrients seemed to correlate with low removal of PhACs. ARGs correlated to organics, nutrients, and some PhACs but not to respective antibiotics. This chapter provides a comprehensive evaluation of the attenuation of PhACs, toxic potency, and ARGs in field CWs. More abundant PhACs in this chapter were selected as representatives for studies in following chapters.

Chapter 3 described the effect of solar irradiation on PhACs in wastewater and the potential to use immobilized $\mathrm{TiO}_{2}$ as a catalyst to achieve photocatalysis of PhACs, which could be applied in CWs. Batch experiments with immobilized $\mathrm{TiO}_{2}$ (photocatalysis) and without (photolysis) were conducted to investigate photodegradation of a mixture of four PhACs under simulated solar irradiation. Photodegradation of all four PhACs followed pseudo-firstorder kinetics, and the kinetic constants of photocatalysis were much higher than those of photolysis. Within 4 days irradiation, photocatalysis resulted in high removal efficiencies for ibuprofen (IBP, 74\%), propranolol (PRO, 100\%), diclofenac (DFC, 100\%), and carbamazepine (CBZ, 76\%), among which the latter three are normally poorly biodegradable in wastewater effluent. When comparing photodegradation of wastewater effluent and deionized water, we found that dissolved natural organic matter (NOM) in the wastewater effluent can enhance PhAC photodegradation by producing reactive radicals (mainly $\bullet \mathrm{OH}$ ). Meanwhile, dissolved NOM inhibited photodegradation, possibly because the dissolved NOM reformed the oxidized PhACs back into parent compounds. With photocatalysis present, toxicity of PhACs decreased and biodegradability of wastewater effluent slightly increased, which may favour subsequent biodegradation. For application, water depth was confirmed as a significant parameter in PhAC photodegradation due to light attenuation by modelling and experimental results. In summary, this chapter proposes to construct a $\mathrm{CW}$ with photocatalysis in an open shallow compartment to remove PhACs more effectively from wastewater effluent. 
Removal of six PhACs were investigated in $\mathrm{CW}$ sediment under different redox conditions in Chapter 4, including aerobic, nitrate reducing, sulfate reducing, and methanogenic conditions. Both batch and column experiments were conducted to explore the effect of electron acceptor availability. Sorption and biodegradation of PhACs showed comparable patterns in batch and column experiments. Sorption behaviour of individual PhACs was more explained by their molecular charge than hydrophobicity. Redox conditions influenced both sorption and biodegradation of PhACs. The least sorption was observed under nitrate reducing conditions. In terms of biodegradation, aerobic conditions showed higher biodegradation efficiencies of PhACs compared to anaerobic redox conditions. Among these anaerobic redox conditions, overall no specific redox condition showed a more effective biodegradation than the others. In general, aerobic conditions demonstrated the highest PhAC removal efficiencies with significant biodegradation of naproxen (NAP), caffeine (CAF), metoprolol (MET), and IBP, and sorption of PRO and MET. For CW application, breakthrough of all tested PhACs is expected after 300 pore volumes and pre-exposure can improve biodegradation capacity of PhACs. This chapter provides insights into the significance of redox conditions in developing and designing CWs for PhACs treatment.

To further elucidate aerobic biodegradation of PhACs in CWs, in Chapter 5 the effect of dissolved NOM and nitrification on aerobic biodegradation of PhACs was studied using enrichment culture originating from the aerobic sediment inoculum . In the $\mathrm{PhAC}$ mixture, CAF, MET, NAP, and IBP were almost completely biodegraded within 35 days batch experiments while biodegradation of PRO, DFC, and CBZ was negligible. Notably, the enriched culture showed a consistent biodegradability order of CAF $>$ MET $>$ NAP $>$ IBP, which may provide an operational strategy for designing hydraulic retention time for removing specific PhACs. Dissolved NOM inhibited CAF biodegradation due to competition between CAF and the easily degradable dissolved NOM. Meanwhile, the dissolved NOM enhanced biodegradation of MET, NAP, and IBP, potentially by either contributing more biomass capable of degrading PhACs, acting as structural analogues, or acting as electron shuttles to stimulate microorganisms to metabolize or co-metabolize PhACs. Thus, addition of dissolved NOM through partial disposal of dead plant biomass could be a strategy to enhance aerobic biodegradation of less biodegradable PhACs in CWs. Finally, nitrification did not influence biodegradation of the readily biodegradable CAF but did inhibit MET biodegradation. Conversely, nitrification enhanced biodegradation of NAP and IBP and mineralization of the PhAC mixture as well as less biodegradable DOM. The enhancement 
effect may be related to co-metabolic conversion by ammonia oxidizing bacteria or enhanced heterotrophic biodegradation under nitrifying conditions. Therefore, nitrification could be maintained in $\mathrm{CWs}$ for biodegradation of $\mathrm{PhACs}$ with certain structures, i.e. with linear alkyl carbons, through introducing continuous ammonium streams. This chapter contributes to the understanding of the role of dissolved NOM and nitrification in PhAC biodegradation, and to the potential optimization of these two factors in $\mathrm{CW}$ application.

To further understand biodegradation of PhACs under anaerobic redox conditions, in Chapter 6 batch experiments were implemented by using enrichment culture originating from specific anaerobic sediment inoculum. Based on chemical and toxicological analyses, biodegradation effectivity of a mixture of six PhACs (CAF, NAP, MET, PRO, IBP, CBZ) and single compounds (CAF, NAP) were evaluated under nitrate reducing, sulfate reducing, and methanogenic conditions. Sulfate reducing and methanogenic conditions showed complete removal of CAF and NAP within six weeks while biodegradation was significantly lower under nitrate reducing conditions. Biodegradation of other four PhACs was less than $20 \%$ under all the three redox conditions. Additionally, production of PhAC intermediates differed under three redox conditions, indicating different biodegradation pathways. When comparing biodegradation of CAF and NAP present as a single compound, or as a mixture with other PhACs, we found no differences in biodegradation efficiencies but in biodegradation intermediates. During biodegradation, toxicity of PhACs and/or their intermediates to Vibrio fischeri was attenuated overall. Chemical and toxicological data showed positive correlations in principle component analysis, by which potentially toxic PhACs and intermediates were screened as candidates for further ecotoxicological hazard assessment. This chapter further indicates the role of redox conditions on PhAC biodegradation not only based on biodegradation rates but also on formation of intermediates.

In Chapter 7, mechanisms of phytoremediation were investigated by observing uptake and transformation of IBP in a wetland plant species. Phragmites australis was exposed to $60 \mu \mathrm{g} / 1$ of IBP under greenhouse conditions for 21 days, during which roots and rhizomes (RR), stems and leaves (SL), and liquid phase samples were collected. We found that IBP was taken up by RR, partly accumulated and partly translocated to SL. Phytodegradation of IBP was confirmed by the occurrence of intermediates: hydroxy-IBP, 1,2-dihydroxy-IBP, carboxy-IBP and glucopyranosyloxy-hydroxy-IBP (IBP-glycoside conjugate) in RR and the first two intermediates in SL tissue. The phytodegradation might relate to enzymes such as cytochrome P450 monooxygenase, which was found to be involved in the production of the two hydroxy 
intermediates. We proposed that transformation of IBP was catalyzed by P450 in the endoplasmic reticulum, then catalyzed by GT in Golgi, followed by further metabolism or storage in vacuoles or cell walls. Moreover, no significant phytotoxicity was observed based on relative growth of plants and stress enzyme activities during phytoremediation. In conclusion, this chapter contributes to understanding and implementing phytoremediation in CWs as an effective treatment technique for removing PhACs.

Chapter 8 demonstrated the fate and distribution of seven PhACs in mesocosm CWs where the individual removal processes were integrated. Additionally, the effect of irradiation via pre-photocatalysis, substrate composition through addition of litter (dead plant biomass, solid NOM), and plants were studied. The mesocosm CWs were started up for PhAC removal within 10 days after $\mathrm{PhAC}$ addition and showed an overall high removal (79-100\% median) for $\mathrm{CAF}$, NAP, MET, PRO, and IBP. In comparison, removal of DFC and CBZ were much lower with only $46 \%$ and $7 \%$, respectively. Mass balance of PhACs was estimated in CWs, from which PhACs were sorbed to substrate (mainly sediment), taken up by plants, degraded through biodegradation and/or phytodegradation. PRO removal was dominated by sorption and other six PhACs were mainly removed by biodegradation and/or phytodegradation. Finally, pre-photocatalysis significantly enhanced PhAC removal except for CAF and IBP, and release the burden of PhAC accumulation in substrate and plant tissues in the following plant compartment. Litter addition in $\mathrm{CW}$ significantly increased removal of PRO, CBZ, and IBP via biodegradation and/or phytodegradation. Plants played an essential and positive role in the removal of all the seven PhACs, resulting from direct phytoremediation, and indirectly enhancing sorption and biodegradation. This chapter provides knowledge to understand removal mechanisms of PhACs with different physicochemical properties in CWs and to potentially enhance the performance of $\mathrm{CWs}$ toward PhAC removal by strengthening the removal processes.

Finally in Chapter 9, outcomes of previous research chapters were summarized and future perspectives were discussed to explore the opportunities and challenges in research and application. We concluded that CWs are promising as post-treatment processes for $\mathrm{PhAC}$ removal due to their high removal capacity, advantages of being cost-efficient and environmentally friendly, and attenuation capacity of toxicity and ARGs. All the individual removal processes in CWs are involved in PhAC removal and they influence each other. Fate of PhACs in CWs was proposed as follows: photodegradation occurs if free water surface is available; a portion of PhACs is sorbed to the substrate, taken up by plants, and retained in 
CWs; another portion is biodegraded by microorganisms, possibly by both aerobic and anaerobic microorganisms, and phytodegraded via enzymatic catalysis. Residual PhACs and biodegradation intermediates flow out the $\mathrm{CW}$ system. Individual PhACs with different physicochemical properties and biodegradability have different removal patterns in various removal processes. For application, we proposed a new concept of $\mathrm{CW}$ with a prephotocatalysis compartment to maximize photodegradation, and other ways to enhance $\mathrm{PhAC}$ removal in CWs for, including litter addition, microbial enrichment, improvement of aeration, ammonium import, optimization of vegetation, and operational parameters, e.g. hydraulic loading rate. For future research, isotope-based technology was suggested to implement for distinguishing the contribution of various degradation processes and identifying the produced intermediates. Toxic intermediates should be identified: first primarily screened by statistical analysis and then identified by effect-directed analysis. Advanced molecular analysis was recommended to screen the potential microorganisms that are functional in PhAC removal. From an application perspective, a robust and sustainable $\mathrm{CW}$ technique with enhanced removal capacity is required for long-term application either in renovation or new construction. 
Appendix 


\section{Acknowledgement}

Finally it is time to write the acknowledgment by sitting in the office quietly and letting my memory flashback to four years ago. I walked through the long corridor from the Axis reception to ETE. A new world came to my eyes: the office, the coffee corner, the secretory, the lab, the supervisors, and the lovely colleagues. I told myself this is a place where I will spend the coming four years and wished I could have a rich experience here. Four year passed without loud reminding. Now it seems that I have to stop and get off the NL-ETE train. Before waving goodbye, I have many thankful words to say.

First of all, I am grateful that the ETE family accepted me and helped me to find myself. During the four years, I harvested fruitful experience which will always be fresh in my memory.

I would like to give the most grateful regards to my supervisors Alette and Nora, and my promotor Huub. You are the "Golden Triangle" in my heart. Alette, the first time I met you through skype I already knew that I want to follow you. I like the way you talk, behave, and treat others. You are always optimistic, encouraging, and inspiring. Staying with you is super comfortable like with families. I received lots of warmth from you which made me less homesick. Thank you for sharing with me your garden. I will be a Dr. of Environmental Technology and a "Dr. of Horticultural Technology". Nora, you participation in my research is an excellent gift for me. Your sharp point of view and observation skills can always enlighten me. During my PhD journey, I could always feel the support from you. Knowing you are there makes me at ease. I really appreciate your open mind for my all sorts of ideas. Huub, you are a warm promotor like Santa with endless ideas in the pocket. Thank you for teaching me how to deal a case from a distinct perspective and how to interpret the findings through precise scientific languages. I admire your scientific attitude, throughout thoughts, and ambitious spirit. I am so grateful that I can meet you Golden Triangle. You never know how satisfying and joyful I am every time after our meeting. I sincerely acknowledge the space and trust you gave me for my research: starting from several keywords to an skeleton, and finally to a vital figure. The experience with you will definitely guide me a right direction to follow along my research journey.

This thesis could not be completed without the support from our superpower ETE laboratory team. Katja, Ilse, Hans, Livio, Jean, Jan Kubiak, Vinnie, Bert, without you I think I could not survive in the lab. You are the smartest guys I have ever met dealing with practical issues. I 
also would like to thank the selfless help from the technicians in other departments. Hennie van Rossum, Gerlinde Vink, Henri Heskamp, and many others, I learned from you the definition of "being a technician". My special thanks to Harry, Thomas ter Laak, and Tim Grotenhuis for offering your inspiring suggestions in my research. Many thanks to the coauthors who contributed their expertise in my research, Tinka, Heike Schmitt, Marco Blokland, Rob Comans, Peter Schröder, Feiran Chen and Christian Huber. Peter and the other colleagues in Helmholtz, I really enjoyed the three months stay with you. Personal gratitude to Joost van den Bulk from Tauw for helping with contacting the water boards and offering kinds of wetland information. Here I also would like to thank my students, Yang, Ziqiu, Xinchen, Rusdi, and Yu. I really appreciate the study experience with you guys.

Of course I would like to acknowledge all the ETEers. Because of you, my life here became more colorful. First I would like to thank our professional and dedicated secretaries, Liesbeth, Gea, Hewin, and other short-term secretaries. It is you who make our work lives well organized. I am glad to be one of our great micropollutant soldiers. Wenbo, Arnoud, Azie, Thomas, Laura, Andrea Brunsch, Andrea Aldas Vargas, Elackiya, it is super nice to work with you in the same team. I am also pleased to meet so many lovely colleagues in the big ETE family: Renata, Kasia, Jan Vreeburg, Grietje, Annemiek, Els, Shokouh, Delaram, Justine, Yvonne, Lucia, Silvi, Leire, Indra, Annemerel, Pradip, Hang, Sanne, Emilius, Rosanne, Viola, Darja, Kasper, Casper, Pim, Koen, Dainis, Tania, Rosa, Gunther, Aken, Laima, Andrii, David, Kanjana, and others who I fail to name. Some of you I know more and some are less, thank you all for the joyful moments and togetherness we ever shared. Finally, I would like to express my special thanks to my Chinese buddies in ETE: Dandan, Momo (Taiwanese), Yujia, Yin, Chunjing, Shiyang, Xuan, Xiaoqian, Ruizhe... and many others who have graduated from ETE (Lei, Zhuobiao, Celia, Yingdi, Bao Park, Jinye, Zhimou, Yifan, Yiyuan, Shengle...). I did enjoy the wonderful times we spent together and you make my stay here more like at home.

Outside of ETE, luckily I have made lots of friends, who also support me along the journey. My best friends Min, Fangjie, Man, and Huicui, I am so grateful that I could have your companion whenever I needed. All the travels, walks, dinners, and secret talks we had together are delightful. Time being with you always flies fast. I know we are connected. Also many thanks to other friends in Wageningen, Ying, Jinghui, Yulian, Yanfei, Yan (Zeng), Liying, Yifan (Zhu), Xiaomei, Annette...I feel very lucky to have you around. Additionally, I would like to thank my travel partners, Yajing and Guanying. The places we visited became 


\section{Acknowledgement}

more attractive because of your companion. I would also like to express my special thanks to my corridor mates, especially for Rianne. The many times dinner and discussion we had add my corridor life more flavor. For my lovely friends outside of the Netherlands, Fen (Dan), Fengying (Jiejie), Yuqian (Mantou), Juan, Yawei, Wanning, Yu (Liu), Qi, Xiang (\&Bin), Fenghui (\&Haifeng), Mingrui...your care and regards from far away distance can always warm my heart. Staying there, I will fly back soon.

Last but not least, I would give my greatest gratitude to my family. Mom and Dad, I cannot describe how proud I am of being your daughter. 谢谢你们给了我无忧无虑的童年, 教我待人 真诚, 为人善良, 做对社会有用的人。是你们满满的爱让我拥有爱的能力, 勇敢无畏, 热爱生 活, 看见斑斓的世界。听过这样一句话, 上帝没有精力照顾所有的世人, 所以创造了爸爸和妈 妈。我想这也是我如此需要你们, 乐而不厌地唤着爸爸妈妈的原因吧。My dearest brother, because of you I feel way more love and happiness. I watch you grow up from the most adorable kid (in the world) to a man. Our childish talks have turned to weekly adult conversations. Until now I still could not help smiling when I recall the touched moments in your childhood, which is the most precious memory in my life. I want you to know that I will always stand by your side. Thank you grandma. 谢谢您的疼爱。每次隔着话筒都能感受到您的 惦念和诨挂。最近总是想起您, 等我回来! Also I would like to thank other family members, especially my cousin, Huimin (laojiahuo). Thanks for the numerous night talks we shared and wonderful time we spent together. My final acknowledgement goes to Mr. Bean. It will be our seven years the day before my defense. I like the way you know what I want to say and I know you know what I want to say. The feeling being with you like walking in the forest with the sunlight shining through trees and a gentle breeze caressing the face. Even though I know that you know, I still want to thank you for always being so supportive in my life. As we expected four years ago, you became a better you and I became a better me. I am looking forward to a better us. 


\section{About the author}

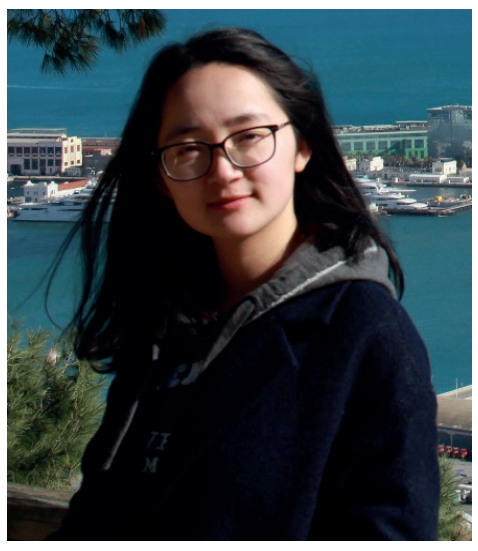

Yujie He was born October16 $6^{\text {th }}, 1991$ in Lu'an, China. In 2006, she graduated as a BSc in Municipal Engineering from Hohai University, Nanjing, China. During the BSc period, she worked on several projects sponsored by National Innovative Fund, including "Effect of shear stress on the physical and chemical characters of aerobic granules", "Investigation of energy saving and emission reduction in cement industry", and "Water pollution and treatment approaches in the textile production chain". For her BSc thesis, she investigated how to enhance removal efficiencies nitrogen and phosphorus in an inverted anaerobic/anoxic/oxic-membrane bioreactor. The thesis was awarded as "Excellent BSc Graduation Thesis" in the university. After graduation, she was recommended to be a postgraduate in Municipal Engineering at Hohai University without entrance examination. Her MSc thesis was aiming to study the distribution of estrogenic endocrine disruptors in different wastewater treatment processes and the adsorption mechanism of these compounds onto the sludge, which was awarded as "Excellent MSc Graduation Thesis" in the university.

In 2013, she won a scholarship offered by China Scholarship Council and started her $\mathrm{PhD}$ at the sub-department of Environmental Technology at Wageningen University. Her PhD research demonstrated the fate and distribution of pharmaceutically active compounds in constructed wetlands with a complex interplay of removal processes, including photodegradation, sorption, biodegradation, and phytoremediation. She will continue with the micropollutant research as a researcher in China. 


\section{List of publications}

He, Y., Sutton, N.B., Rijnaarts, H.H.M., Langenhoff, A.A.M. Degradation of pharmaceuticals in wastewater using immobilized $\mathrm{TiO}_{2}$ photocatalysis under simulated solar irradiation. Applied Catalysis B: Environmental 2016, 182, 132-141.

He, Y., Langenhoff, A.A.M., Sutton, N.B., Rijnaarts, H.H.M., Blokland, M.H., Chen, F., Huber, C., Schröder, P. Metabolism of ibuprofen by Phragmites australis: uptake and phytodegradation. Environmental Science \& Technology 2017, 51 (8), 4576-4584.

He, Y., Sutton, N.B., Rijnaarts, H.H.M., Langenhoff, A.A.M. Pharmaceutical biodegradation under three anaerobic redox conditions evaluated by chemical and toxicological analyses. Science of the Total Environment 2017. DOI 10.1016/j.scitotenv.2017.07.219.

He, Y., Nurul, S., Schmitt, H., Sutton, N.B., Murk, T.A.J., Blokland, M. H., Rijnaarts, H.H.M., Langenhoff, A.A.M. Evaluation of attenuation of pharmaceuticals, toxic potency, and antibiotic resistance genes in constructed wetlands treating wastewater effluents. Submitted.

De Wilt, A.*, He, Y.*, Sutton, N.B., Langenhoff, A.A.M., Rijnaarts, H.H.M. Influence of redox conditions on sorption and biodegradation of pharmaceuticals in sediment batch and column experiments ( ${ }^{*}$ co-first author). Submitted.

He, Y., Langenhoff, A.A.M., Comans, R.N.J., Sutton, N.B., Rijnaarts, H.H.M. Effects of dissolved organic matter and nitrification on biodegradation of pharmaceuticals in aerobic enrichment cultures. Submitted.

He, Y., Sutton, N.B., Lei Y., Rijnaarts, H.H.M., Langenhoff, A.A.M. Fate and distribution of pharmaceuticals in mesocosm constructed wetlands. Submitted. 


\section{SENSE}

Netherlands Research School for the

Socio-Economic and Natural Sciences of the Environment

\section{I P L O M A}

For specialised PhD training

The Netherlands Research School for the Socio-Economic and Natural Sciences of the Environment

(SENSE) declares that

\section{Yujie He}

born on 16 October 1991 in Anhui, China

has successfully fulfilled all requirements of the

Educational Programme of SENSE.

Wageningen, 10 October 2017

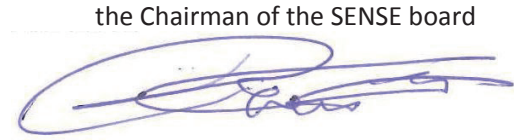

Prof. dr. Huub Rijnaarts the SENSE Director of Education

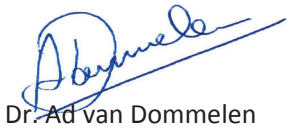

The SENSE Research School has been accredited by the Royal Netherlands Academy of Arts and Sciences (KNAW)

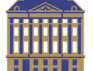

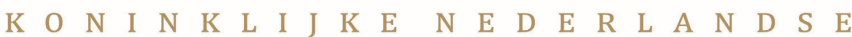

$\begin{array}{lllllllllllllllllllllllll}\text { A } & \text { K } & \text { A } & \text { D } & \text { E } & \text { M } & \text { I } & \text { E } & \text { V } & \text { A } & \text { N } & \text { W } & \text { E } & \text { T } & \text { E } & \text { N } & \text { S } & \text { C } & \text { H } & \text { A } & \text { P } & \text { P } & \text { E } & \text { N }\end{array}$ 


\section{(suse)}

The SENSE Research School declares that Ms Yujie He has successfully fulfilled all requirements of the Educational PhD Programme of SENSE with a work load of $46.7 \mathrm{EC}$, including the following activities:

\section{SENSE PhD Courses}

- Environmental research in context (2014)

- SENSE writing week (2015)

- Research in context activity: 'Co-organizing the CHI-NED 4D Dialogue Seminar and a PhD study trip to China' (2016)

\section{Other PhD and Advanced MSc Courses}

- Project and time management, Wageningen University (2013)

- Information literacy including EndNote introduction, Wageningen University (2013)

- Presentation skills, Wageningen University (2014)

- Teaching and supervising thesis students, Wageningen University (2014)

- Techniques for writing and presenting a scientific paper, Wageningen University (2014)

- Competence assessment, Wageningen University (2014)

- Francqui inaugural and further lectures by David Sedlak on Water 4.0, Centre Environmental Science and Technology, Belgium (2015)

o $\quad 3^{\text {rd }}$ Water_2020 Training School 'Fate, treatment, environmental and economic impacts of micropollutants and emissions', University of Santiago de Compostela, Spain (2015)

o Career perspectives, Wageningen University (2016)

External training at a foreign research institute

- Research exchange COST Action 'Conceiving Wastewater Treatment in 2020 - Energetic, environmental and economic challenges (Water_2020)', Helmholtz Institute, Germany (2015)

- PhD study trip to Tsinghua University, Chinese Academy of Sciences, Tongji University and etc., China (2016)

\section{Management and Didactic Skills Training}

o Supervising five MSc students (2014-2017)

o Participating in WUR Library committee, Wageningen UR (2015-2017)

\section{Selection of Oral Presentations}

- Removal of pharmaceuticals by photocatalysis under simulated solar irradiation. $9^{\text {th }}$ IWA Micropol \& Ecohazard Conference, 22-25 November 2015, Singapore

SENSE Coordinator PhD Education

Dr. ing. Monique Gulickx

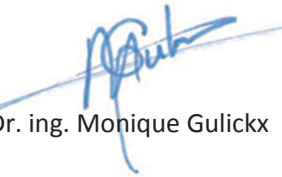


The support provided by China Scholarship Council (CSC) for the research of Yujie He at Wageningen University is kindly acknowledged.

Financial support from Wageningen University for printing this thesis is gratefully acknowledged.

Cover painting drawn by Louise van Eenennaam-Mulder 